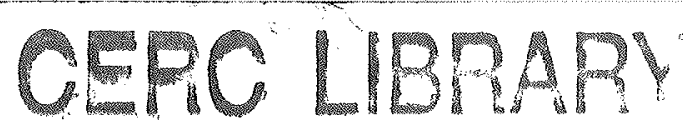

US Army Corps of Engineers
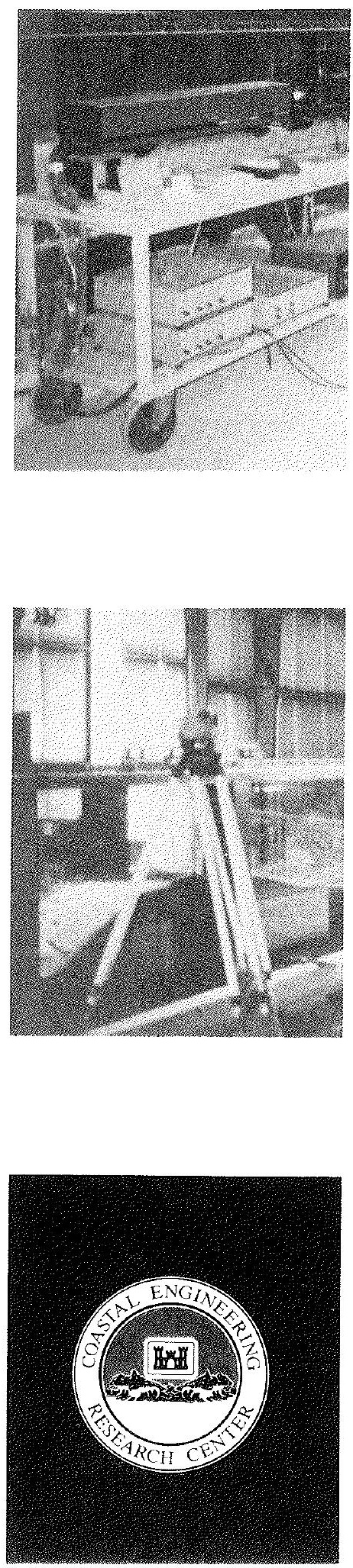

\section{IRREGULAR WAVE-INDUCED VELOCITIES IN SHALLOW WATER}

\author{
by \\ Nels John Sultan \\ Ocean Engineering Department \\ Texas A\&M University \\ College Station, Texas $\quad 77843-3136$
}

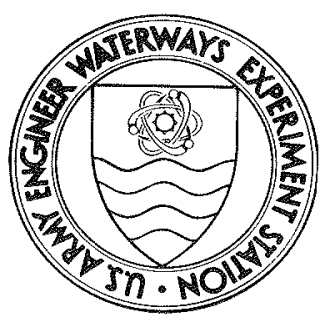

September 1992

Final Report

Approved For Public Release; Distribution Is Unlimited

Prepared for DEPARTMENT OF THE ARMY US Army Corps of Engineers

Washington, DC 20314-1000

Under Work Unit 32684 
Destroy this repont when no longer needed. Do not retum it to the originator.

The findings in this report are not to be-construed as an official

Department of the Army position unless so designated by other authorized documents.

The contents of this report are not to be used for advertising, publication, or promotional purposes. Citation of trade names does not constitute an official endorsement or approval of the use of such commercial products. 


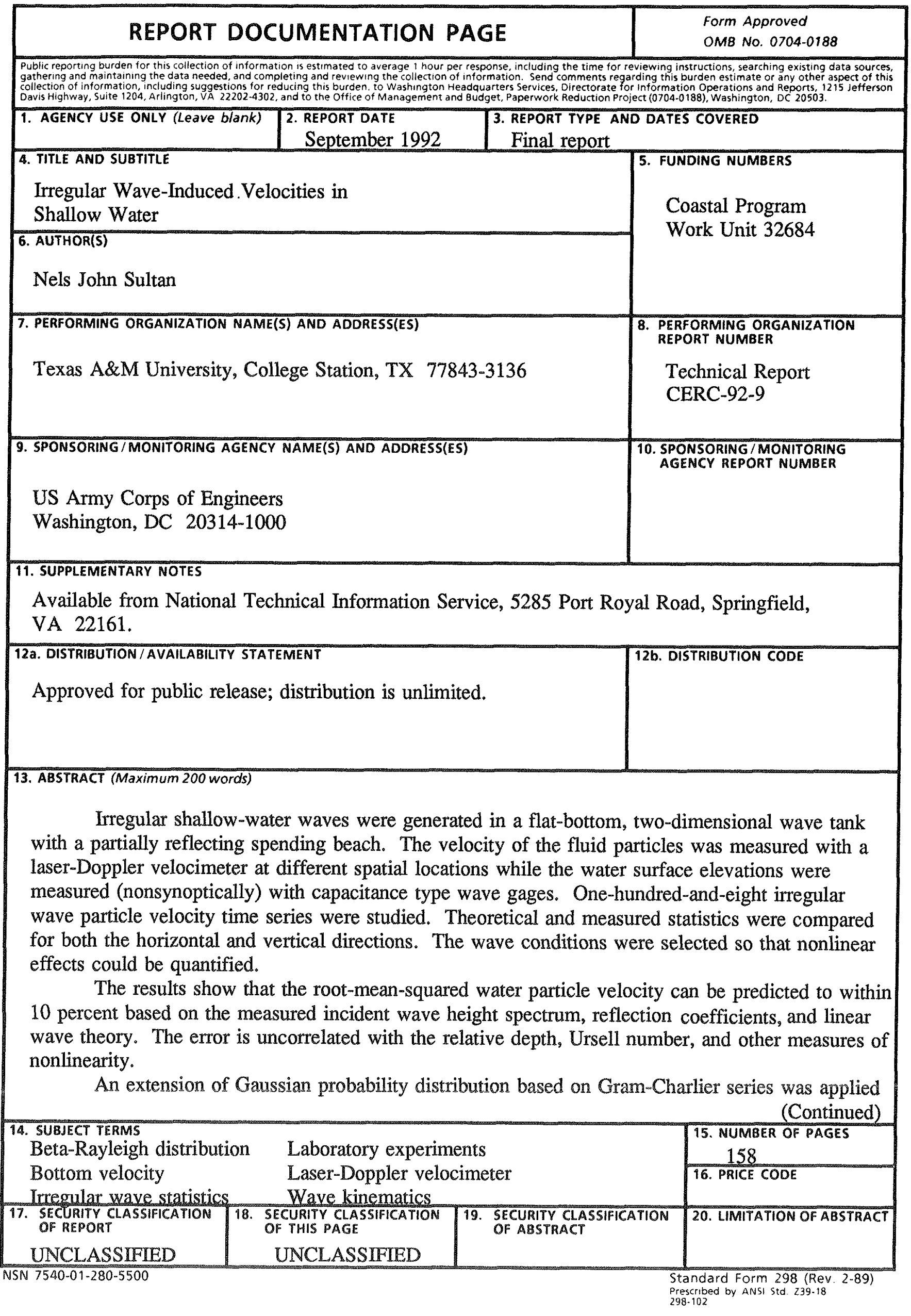




\section{3. (Concluded).}

to the entire velocity time series. This extended distribution did not fit the measured data better than the usual Gaussian distribution.

A probability distribution function called the Beta-Rayleigh distribution was applied to peak velocities at both mid-depth and near the bottom in the water column. A 15-percent improvement relative to the classical Rayleigh distribution is observed for the case of near-bottom vertical velocities. Near-bottom horizontal velocities show only 1- to 2-percent improvement. The parameters of the distribution are empirically correlated with parameters of the incident wave spectrum. 


\section{PREFACE}

This study was performed in partial fulfillment of the requirements for the Master of Science degree in Ocean Engineering from Texas A\&M University. Work was performed at Texas A\&M University, College Station, Texas, and at the Coastal Engineering Research Center (CERC), US Army Engineer Waterways Experiment Station (WES), Vicksburg, Mississippi. The work herein was authorized as a part of the Civil Works Research and Development Program by the Headquarters, US Army Corps of Engineers (HQUSACE), and was performed under the Scour Holes at Ends of Structures Work Unit 32684, which is in the Harbor Entrances \& Coastal Channels Program. Messrs. John H. Lockhart, Jr.; Barry W. Holiday; David A. Roellig; and John G. Housley are HQUSACE Technical Monitors. Ms Carolyn M. Holmes is Program Manager for the Harbor Entrances \& Coastal Channels Program.

The study was conducted over the period May 1991 through Nov 1991 by Mr. Nels John Sultan, Department of Ocean Engineering, Texas A\&M University, while a graduate student at Texas A\&M University. Work was performed under supervision of Dr. Steven A. Hughes, visiting Assistant Professor at Texas A\&M University and Senior Research Engineer at CERC, and Dr. Robert E. Randall, Associate Professor at Texas A\&M University. Work performed at CERC was under the general administrative supervision of Mr. C. E. Chatham, Chief, Wave Dynamics Division, Mr. Charles C. Calhoun, Assistant Director, CERC, and Dr. James R. Houston, Director, CERC. Messrs. Raymond C. Herrington and David Daily provided assistance during the laboratory phase of the experiments, and Ms. Debra R. Green and Mr. John M. Heggins assisted in data analysis.

At the time of publication of this report, Director of WES was Dr. Robert W. Whalin. Commander and Deputy Director was COL Leonard G. Hassell, EN. 


\section{TABLE OF CONTENTS}

Page

PREFACE

i

LIST OF TABLES

iv

LIST OF FIGURES

$\mathrm{V}$

1 INTRODUCTION

1.1 Present Status of the Question ................ 1

1.1.1 Velocity Probability Distribution ........... 2

1.1.2 Peak Velocities ................. 3

1.1.3 Other Investigations .............. 4

2 EXPERIMENTAL PROCEDURES

2.1 Experimental Plan ................... 15

2.2 Computer Output .................... 20

2.3 Wave Signal Generation ................ 20

3 THE ROOT-MEAN-SQUARE VELOCITY 24

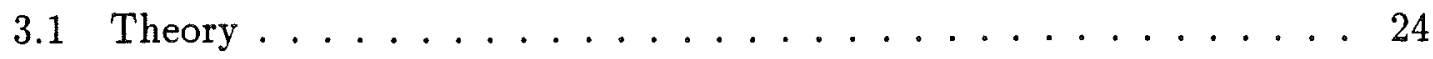


3.2 Experimental Results . . . . . . . . . . . . . 26

3.3 Laboratory Effects . . . . . . . . . . . . . . 35

4 VELOCITY DISTRIBUTION 45

4.1 Gram-Charlier Distribution . . . . . . . . . . . 45

5 PEAK VELOCITY DISTRIBUTION 53

5.1 The Beta-Rayleigh Distribution . . . . . . . . . . 53

5.2 Experimental Results . . . . . . . . . . . . . 56

5.3 Parameterization ................ 63

5.3 .1 Maximum Velocity . . . . . . . . . . 67

5.3.2 Root-Mean-Square and Root-Mean-Quad Peak Velocity . . . 71

6 SUMMARY AND CONCLUSIONS 77

$\begin{array}{lr}\text { REFERENCES } & 80\end{array}$

APPENDIX I: SUPPLEMENTAL GRAPHS 83

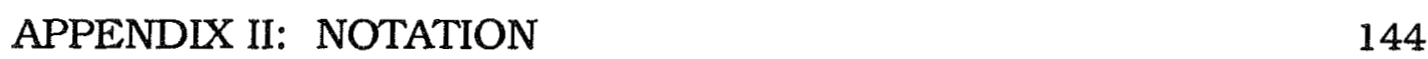


Table

\section{LIST OF TABLES}

1 Experimental Plan .................. 15

2 Summary - Individual Time Series . . . . . . . . . . . . 17

3 Experiment Repeatability ............... 42

4 Beta-Rayleigh Distribution, Mean \%-RMS Error . . . . . . 58

5 Root-Mean-Square and Root-Mean-Quad Curve Fitting . . . . . 73 
Figure

\section{LIST OF FIGURES}

1 Wave Tank Photographs $\ldots \ldots \ldots \ldots \ldots \ldots$

2 Equipment Close-up Photographs . . . . . . . . . . 9

3 Wave Tank-Schematic . . . . . . . . . . . . . 10

4 Computer Output - Water Particle Velocity Time Series Analysis . 12

$5 \quad$ Measured Incident and Reflected Wave Spectra $\ldots \ldots \ldots \ldots$

6 Velocity Time Series-Run No. $19 \ldots \ldots \ldots$. . . . . . . 21

$7 \quad u_{r m s}$ Measured/Predicted vs. Relative Depth . . . . . . 27

$8 u_{r m s}$ Measured/Predicted vs. Ursell No. . . . . . . . . . 28

$9 \quad w_{r m s}$ Measured/Predicted vs. Relative Depth . . . . . . . 29

$10 w_{r m s}$ Measured/Predicted vs. Ursell No. . . . . . . . . 30

11 Predicted $u_{r m s}$ Velocity vs. X-coordinate (Time Series No. 9) . . . 33

12 Predicted $u_{r m s}$ Velocity vs. X-coordinate (Time Series No. 69) . . 34

$13 \%$-Range of $u_{r m s}$ Predicted as a Function of X, (Random Phase Angle) vs. Relative Depth . . . . . . . . . . . . . 36

14 Reflection Coefficient vs. $H / h$, Monochromatic Waves . . . . . . 37

15 Reflection Coefficient vs. $H_{m o} / h$, Irregular Waves $\ldots \ldots \ldots . . .38$

16 Reflection Coefficient vs. Relative Depth, Irregular Waves . . . . . 39

17 Reflection Coefficient vs. Peak Spectral Period, Irregular Waves . . 40

18 Number of Waves Counted for Water Elevation Time Series and Water Particle Velocity Time Series . . . . . . . . . . . . . 44 
Figure

19 Horizontal Velocity Probability Distribution . . . . . . . . . 48

20 Vertical Velocity Probability Distribution . . . . . . . . . . 49

$21 \%$-Root-mean-square Error, Gram-Charlier Distribution vs. $H_{m o} / h \quad 50$

22 The Effect of Varying Filter Bandwidths on Fitting the GramCharlier Distribution to Horizontal Velocities, Time Series No. 37 . 51

23 The Effect of VArying Filter Bandwidths on Fitting the GramCharlier Distribution to Vertical Velocities, Time Series No. 37 . . 52

24 Velocity Peaks Probability Distribution ............ 57

$25 \%$-Root-mean-square Error, Beta-Rayleigh Distribution and Rayleigh Distribution, Negative Horizontal Direction, Mid-depth . . . . . . 59

$26 \%$-Root-mean-square Error, Beta-Rayleigh Distribution and Rayleigh Distribution, Negative Horizontal Direction, Near Bottom . . . . 60

27 The Effect of Varying Filter Bandwidths on the Probability Distribution of Horizontal Velocity Peaks . . . . . . . . . . 61

28 The Effect of Varying Filter Bandwidths on the Probability Distribution of Vertical Velocity Peaks ............ 62

29 Theoretical Ratios, Beta-Rayleigh Distribution, Horizontal Direction 64

30 Theoretical Ratios, Beta-Rayleigh Distribution, Vertical Direction . 65

31 Horizontal Velocity Peaks Probability Distribution, $u_{r m s}$ Only Pa-

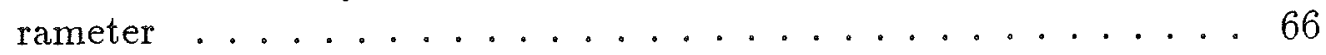

32 Maximum Velocity Measured/Predicted . . . . . . . . 69

33 Horizontal Velocity Peaks Probability Distribution . . . . . . 70

34 Mean Horizontal Velocity Peaks .............. 74 
Figure

35 Parameterization of Beta-Rayleigh Distribution, Horizontal Direction 75

36 Parameterization of Beta-Rayleigh Distribution, Vertical Direction . 76

$37 u_{r m s}$ Measured/Predicted vs. Peak Spectral Period . . . . . . . 84

$38 u_{r m s}$ Measured/Predicted vs. Spectral Significant Wave Height . . . 85

$39 u_{\tau m s}$ Measured/Predicted vs. Water Depth . . . . . . . . . 86

$40 \quad u_{r m s}$ Measured/Predicted vs. $Z / h \ldots \ldots \ldots 7$

$41 u_{r m s}$ Measured/Predicted vs. $H_{m o} / h \ldots \ldots . \ldots 8$

$42 \quad u_{r m s}$ Measured/Predicted vs. $H_{m o} / g T_{p}^{2} \ldots \ldots \ldots . \ldots$

$43 \quad u_{r m s}$ Measured/Predicted vs. $Z H_{m o} / h^{2} \ldots \ldots \ldots$

$44 w_{r m s}$ Measured/Predicted vs. Peak Spectral period . . . . . . . 91

$45 w_{\text {rms }}$ Measured/Predicted vs. Spectral Significant Wave Height . . . 92

$46 \quad w_{r m s}$ Measured/Predicted vs. Water Depth . . . . . . . . . 93

$47 w_{r m s}$ Measured/Predicted vs. $Z / h \ldots \ldots . \ldots \ldots 4$

$48 w_{r m s}$ Measured $/$ Predicted vs. $H_{m o} / h \ldots \ldots \ldots$

$49 w_{r m s}$ Measured/Predicted vs. Ursell No. (without outliers) ... . . 96

$50 \quad u_{r m s}$ Measured $/$ Predicted vs. $Z H_{m o} / h^{2} \ldots \ldots \ldots 7$

$51 \%$-Range of $u_{r m s}$ Predicted as a Function of X, (Measured Phase Angle . . . . . . . . . . . . . . . . . . 98

$52 \%$-Range of $w_{\text {rms }}$ Predicted as a Function of X, (Random Phase Angle . . . . . . . . . . . . . . . . . . . 99 
Figure

$53 \%$-Range of $w_{r m s}$ Predicted as a Function of X, (Measured Phase

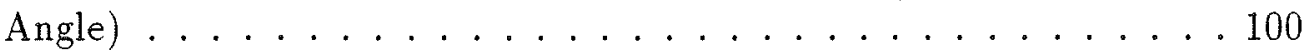

54 No. Waves Counted, Horizontal Direction . . . . . . . . . . 101

55 No. Waves Counted, Vertical Direction . . . . . . . . . . 102

$56 \%$-Root-mean-square Error Beta-Rayleigh Distribution, Positive Horizontal Direction ................. . . 103

$57 \%$-Root-mean-square Error Beta-Rayleigh Distribution, Positive Vertical Direction . . . . . . . . . . . . . . . 104

$58 \%$-Root-mean-square Error Beta-Rayleigh Distribution, Negative Vertical Direction . . . . . . . . . . . . . . 105

59 The Effect of Varying Filter Width, Horizontal Velocity Peaks, Time Series No. 170 . . . . . . . . . . . . . . 106

60 The Effect of Varying Filter Width, Horizontal Velocity Peaks, Time Series No. 19 ................. . . 107

61 The Effect of Varying Filter Width, Horizontal Velocity Peaks, Time Series No. 70 ..................... 108

62 The Effect of Varying Filter Width, Vertical Velocity Peaks, Time Series No.170 . . . . . . . . . . . . . . . . 109

63 The Effect of Varying Filter Width, Vertical Velocity Peaks, Time Series No. 19 . . . . . . . . . . . . . . . 110

64 The Effect of Varying Filter Width, Vertical Velocity Peaks, Time Series No. 70 ............................ 111

65 Vertical Velocity Peaks, $w_{r m s}$ Only Parameter ......... 112

66 Maximum Velocity Measured/Predicted vs. Relative Depth . . . . 113

67 Vertical Velocity Peaks Probability Distribution, 2x Usual $W_{\max }$. 114 
68 Vertical Velocity Peaks Probability Distribution, $0.5 x$ Usual $W_{\max } .115$

69 Parameterization of Beta-Rayleigh Distribution, $U_{r m s} \ldots \ldots 116$

70 Parameterization of Beta-Rayleigh Distribution, $U_{r m q} \ldots \ldots 117$

71 Parameterization of Beta-Rayleigh Distribution, $W_{r m s} \ldots \ldots 118$

72 Parameterization of Beta-Rayleigh Distribution, $W_{r m q} \ldots \ldots 119$

73 Time Series No. $9 \ldots \ldots \ldots . \ldots . \ldots . \ldots 120$

74 Time Series No. $153 \ldots \ldots \ldots \ldots$. . . . . . . . . . . . . . . . . .

75 Time Series No. $18 \ldots \ldots . \ldots . \ldots . \ldots . \ldots 22$

76 Time Series No. $19 \ldots \ldots . \ldots \ldots$. . . . . . . . . . . . . . . . .

77 Time Series No. $25 \ldots \ldots \ldots \ldots \ldots \ldots$

78 Time Series No. $28 \ldots \ldots \ldots \ldots . \ldots \ldots$

79 Time Series No. $29 \ldots \ldots \ldots \ldots$

80 Time Series No. $35 \ldots \ldots . \ldots . \ldots . \ldots 127$

81 Time Series No. $38 \ldots \ldots . \ldots \ldots$

82 Time Series No. 39 . . . . . . . . . . . 129

83 Time Series No. $45 \ldots \ldots . \ldots . \ldots . \ldots 130$

84 Time Series No. $48 \ldots \ldots \ldots 131$

85 Time Series No. $49 \ldots \ldots . \ldots \ldots 2$

86 Time Series No. $55 \ldots \ldots . \ldots \ldots \ldots 33$ 


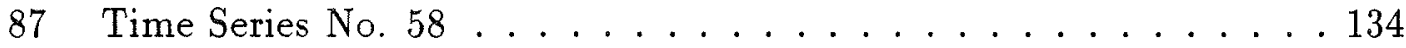

88 Time Series No. $59 \ldots \ldots \ldots$. . . . . . . . . . . . . . . . . . . . . . . . .

89 Time Series No. $65 \ldots \ldots \ldots$. . . . . . . . . . . . 136

90 Time Series No. 68 . . . . . . . . . . . 137

91 Time Series No. $69 \ldots \ldots \ldots$. . . . . . . . . . . . . . . . . . . .

92 Time Series No. $75 \ldots \ldots$. . . . . . . . . . . . . . . . . . . . . . . . .

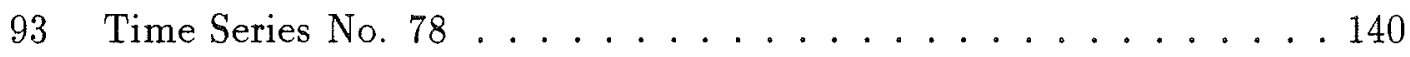

94 Time Series No. $79 \ldots \ldots$. . . . . . . . . . . . . 141

95 Time Series No. $85 \ldots \ldots . \ldots . \ldots . \ldots . \ldots 2$

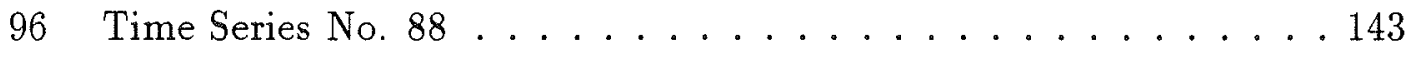




\section{INTRODUCTION}

Irregular wave kinematics is of fundamental importance to many coastal engineering phenomena. Sediment transport and scour are two processes highly

dependent on a good understanding of fluid particle velocities. Incipient motion criteria are usually a function of fluid velocity. As well, the phenomena of sediment transport under wave action alone is believed to be partly a result of the nonsymmetrical shape of the wave profile and associated nonlinear velocities. In this thesis the nonlinear effects of the nonsymmetrical wave profile on the water particle velocities are investigated.

One objective of this research is to develop empirical equations for determining the parameters of probability distribution functions for particle velocities. The probability distribution functions for both peak velocities and for the entire velocity time series are investigated. Also, all analyses are completed for both the horizontal and vertical components of velocity.

Another objective is to investigate a purely theoretical expression for the rootmean-squared velocity, $\left(u_{r m s}\right.$ and $\left.w_{r m s}\right)$, of the entire velocity time series. The expression is based on the incident wave height spectrum, reflection coefficients, and linear wave theory. The range of validity and the effect of nonlinearities are studied. The expressions are simply the standard deviation of the velocity and are useful parameters in studies of turbulence and sediment transport.

It is intended that this research provide a firm foundation for further studies related to sediment transport and seafloor scour. For this reason, the experiments have been performed in the lower half of the water column in a tank with a horizontal bed. Thus, the results reported here do not necessarily apply to wave kinematics near the free surface.

\subsection{Present Status of the Question}

Previous investigations in wave induced fluid velocities show generally "good" agreement with linear theory. Dean (1990) reviews the state of the art in water wave kinematics. For regular (monochromatic) waves it appears that current theories are adequate to within the limit of errors inherent in the laboratory equipment. This limit is suggested by Dean to be approximately $5 \%$. For irregular waves the

Citations in the thesis will be consistent with those of the Journal of Waterway, Port, Coastal and Ocean Engineering, ASCE. 
existing literature is somewhat more vague. Dean (1990) reports that "... available wave theories seem to represent adequately the measurements" for the study of kinematics below the trough elevation.

The previous investigations described below summarize the literature most relevant to this thesis. Surprisingly, much of the work in this thesis has not been done before despite being of a fundamental nature.

This thesis further develops the ideas in the paper Estimating Wave-Induced Bottom Velocities at a Vertical Wall (Hughes, 1992). In this paper, the general theoretical expression for the root-mean-squared velocity of the entire time series was simplified to the case of irregular waves in front of a vertical wall. The expression was validated using laboratory measurements. However, no range of validity or empirical augmentation for nonlinearities was determined. Also, the paper only investigated horizontal velocities and assumed a perfectly reflecting vertical wall.

One paper closely related to this investigation was that by Soulsby (1987) where a method was presented for calculating the root-mean-squared bottom orbital velocity given the wave height, period and water depth and any one of the following spectral forms; JONSWAP, Pierson-Moskowitz, Bretschneider, ISSC or ITTC. The method of calculating $u_{r m s}$ is identical to that by Hughes, except it does not allow for wave reflections. This paper is strictly theoretical and no experiments were performed. Thus, the limits of nonlinearities were not studied, and no experimental data were presented to illustrate the validity of the theoretical expression. The paper only considered horizontal near-bottom velocities.

\subsubsection{Velocity Probability Distribution}

Longuet-Higgins (1963) derived the probability density function of the water surface elevation using the Gram-Charlier series to expand the Gaussian probability density function. This thesis applies this expanded distribution to fluid particle velocities instead of wave elevations. Ochi (1982) presents a review of recent advances in the stochastic analysis of random seas. He notes that the first term of the extended distribution, henceforth called the Gram-Charlier distribution, represents the Gaussian waves and the higher order terms are attributed to the nonlinear, non-Gaussian wave characteristics. The author notes that there does not seem to be any strict theoretical proof that the higher order terms in the series are necessarily a result of wave nonlinearities. It seems that this explanation is derived through a process of elimination. That is, it can be proved mathematically, (Longuet-Higgins 1963), that purely linear waves will have a Gaussian distribution. Therefore, any deviation from a Gaussian distribution must be attributed to 
wave nonlinearities.

Ochi (1982) discusses a series of experiments by Honda and Mitsuyasu (1976) comparing the Gram-Charlier distribution with measurements of wave elevations, particle velocities and particle accelerations for the entire time series. (Unfortunately, the original paper is a report from a Japanese university and not available to the author.) Reportedly, very good agreement is found for the case of wave elevations but not for wave accelerations. Given the close relationship between acceleration and velocity it is reasonable to suppose that poor agreement was also found for the case of wave velocities. Poor agreement might be attributable to the quality of measurement techniques in 1975.

Ochi (1982) also covers two purely theoretical works concerning the probability distribution of non-Gaussian wave elevations. Tayfun (1980) uses second order Stokes theory and the assumption of a narrow banded wave spectrum to derive a function that requires numerical integration for its solution. The distribution is compared to simulated data. St. Denis (1975) uses third order Stokes theory and a joint probability distribution function of wave heights and wave periods. The wave heights and periods are selected at random from these two distributions. Both Tayfun's and St. Denis's methods may be applicable to wave particle velocities but such an investigation is beyond the scope of this thesis.

Anastasiou, Tickell and Chaplin (1982) reported that the statistical distribution of both horizontal and vertical particle velocities follow a Gaussian form for points measured more than half a significant wave height below the mean water level. The measurements of velocities were made with a laser Doppler velocimeter (LDV) under irregular waves in a wave tank. However, their study focused on velocities in the top half of the water column and in the wave crest.

\subsubsection{Peak Velocities}

The literature concerning the probability distribution of time series peaks (as opposed to the entire time series) is more extensive. The Beta-Rayleigh distribution applied in this thesis to velocity time series peaks was originally developed for shallow water wave heights (Hughes and Borgman 1987 and Borgman 1986). Hughes and Borgman reported a "reasonable fit" to measured wave height field data.

Ochi (1982) and Hughes and Borgman (1987) discuss several proposed wave height distributions. Studies have been done that use the Gluhovski distribution, 
the Ibrageemov distribution and the Weibull distribution. A number of studies have tried modifications to the Rayleigh distribution and the Gamma distribution. However, all the theoretical studies look exclusively at the distribution of wave heights rather than peak velocities. The author is unaware of any attempts at fitting a probability distribution to peak particle velocities.

Vis (1980) compares theoretical and measured orbital velocities for irregular waves in a wave tank. Measurements were made with a LDV and compared with predicted velocities based on linear wave theory and the synoptically measured water level elevations. Both horizontal and vertical components of velocity were analysed. An important observation was that measured horizontal velocities under the trough appeared greater in magnitude than horizontal velocities under the crest. Linear theory predicts symmetrical velocities under the crest (particle motion in the positive direction) and under the trough (negative direction). The measured vertical velocities appeared to be more symmetrical. Also noted was the somewhat surprising result that linear wave theory more accurately predicted velocities near the free surface (just beneath the deepest trough) than at mid-depth. In addition, linear theory more accurately predicted bottom velocities than those at mid-depth, as expected. Vis also notes that peak velocities appeared to be nearly Rayleigh distributed.

Daemrich, et. al. (1980, 1982) also compared theoretical and measured orbital velocities for irregular waves in a wave tank. However, these measurements were made with inductive type and impeller type probes rather than with a LDV. Both horizontal and vertical peak velocities were analysed. This study observed that measured peak velocities under the trough were higher than measured peak velocities under the crest, as was observed by Vis (1980). Neither Vis nor Daemrich, et. al. quantified this discrepency.

\subsubsection{Other Investigations}

Lee, Skjelbreia and Raichlen (1982) compared theoretical and measured water particle velocities for solitary waves in a wave tank. Measurements were made with a LDV for both the horizontal and vertical components of velocity. Existing theories of varying order were found to agree well with the measurements.

Battjes and Van Heteren (1984) compared field measurements of fluid particle velocities with linear wave theory. The velocity meter measured all three components of velocity using acoustic pulses. Water surface elevations were measured with a resistance type step gage. Possible errors in the velocity measurements were reported not to exceed $\pm 5 \%$. Measured vertical velocities agreed very well 
with linear theory predictions. Measured horizontal velocities were systematically lower than predicted velocities by an estimated $5 \%$, which is within the range of possible measurement error. Measurements of water particle velocities and water surface elevations were not synoptic.

Bullock and Short (1985) compared theoretical and measured water particle velocities for regular (monochromatic) waves in a wave tank. Both the horizontal and vertical components of water particle velocity were measured with a LDV. (The two components could not be measured simultaneously with this particular LDV.) Comparison with Stokes' theories of various orders revealed that water particle velocities were not always well predicted. Errors of over $10 \%$ are possible in the magnitude of the first harmonic of velocity and greater errors may be expected for higher harmonics. A significant mean horizontal velocity was measured with a magnitude of up to $20 \%$ of the first harmonic velocity. The investigation also noted the importance of laboratory effects such as reflection from the spending beach and the existence of standing waves in the wave tank.

Swan (1990) measured mean horizontal velocities (also known as drift velocity or convection) with a LDV in a wave tank. The development of a drift velocity over time was measured. In the early stages the drift velocity was very similar to Stokes' solution for irrotational Lagrangian mass transport. As time progressed the magnitude of the backflow steadily increased until the velocities were three to four times larger than the initial mean velocity. The time scale for this development was on the order of one to ten hours and is therefore not of immediate relevance to this thesis. However, the investigation did emphasize the importance of laboratory effects.

Hansen (1990) analysed existing experimental data on regular waves in the surf zone (wave breaking conditions). He noted that measured horizontal water particle velocities are virtually independent of elevation in the surf zone. Also, linear theory greatly overestimates the magnitude of particle velocity for this condition.

Kim, Randall, Krafft, and Boo (1990) measured the kinematics of large transient waves with a LDV. The measured velocities under the crest were compared with theoretical predictions based on an "equivalent" Stokes wave. It was concluded that measured transient wave velocities were much more severe than predicted velocities above the still water level and somewhat less severe below the still water level.

Zhang, Randall, and Spell (1991) conducted a more general study of wave crest kinematics. Velocities were measured with a LDV and compared with theoretical values based on the following three common approximate methods; Wheeler 
stretching, linear extrapolation, and vertical extrapolation. It was concluded that the prediction accuracy of each method depends on the bandwidth of the wave spectrum. For narrow bandwidths, linear extrapolation is a better predictor. For broad bandwidths, Wheeler stretching is a better predictor. 


\section{EXPERIMENTAL PROCEDURES}

Laboratory experiments were performed in a flat bottom two-dimensional wave tank at the Coastal Engineering Research Center in Vicksburg, Mississippi. The dimensions of the tank are $46 \mathrm{~cm}$ wide by approximately $46 \mathrm{~m}$ long, (Figures 1a and 3). The height of the walls is $90 \mathrm{~cm}$. A gently sloping beach covered with wave absorbing material was at one end of the tank opposite the wave-maker to minimize wave reflections. Three layers of horsehair mats were used on the spending beach with a total thickness of approximately $7 \mathrm{~cm}$. The slope of the beach was $1: 30$.

An array of three surface piercing capacitance type wave gauges were placed in the middle of the wave tank, (Figures 2a and 3). Each gauge measured the water surface elevation as a function of time. Incident and reflected wave height spectra were calculated from these measurements using the method of Goda and Suzuki (1976). The wave gauges were calibrated before each day's measurements. The estimated error for the gauge calibration is $\pm 0.1 \mathrm{~cm}$ (Briggs 1991). Other sources of error in the measurement of water elevations are due to the internal electronics of the VAX computer and are assumed negligible.

Water particle velocities were obtained using a laser Doppler velocimeter (LDV), which permits non-intrusive measurements of fluid velocities. The LDV is powered by an Innova 70-2 continuous Argon-Ion laser manufactured by Coherent, Inc., Palo Alto, California. The wavelength of the emitted light is $488.0 \mathrm{~nm}$ and $514.5 \mathrm{~nm}$, one for each component of velocity being measured. The laser permits high sampling rates for velocities in both the horizontal and vertical directions. The maximum power level is $2 \mathrm{~W}$. The output beam diameter is $1.5 \mathrm{~mm}$ for 514.5 $\mathrm{nm}$ wavelength. The laser is mounted on an optical bench and can be seen in the lower left corner of Figure 1b. Also visible in Figure $1 \mathrm{~b}$ is the microcomputer used to record and analyze the water particle velocity time series. The actual laser beams are visible in the lower right corners of Figures $2 a$ and $2 b$ as the beams emerge from the fiber optic probe. Velocities were measured at the laser beam crossing point, which for these experiments was located approximately $7 \mathrm{~cm}$ from the inside face of the glass side wall. Seven centimeters from the face of wall is well outside the boundary layer since for steady flow on a smooth surface the boundary layer thickness is on the order of $1 \mathrm{~mm}$ (Munson, Young, and Okiishi 1984).

The physics of laser doppler velocimetry has been well documented in other sources (Goldstein 1983). In simple terms, fluid particle velocities are measured when a small particle suspended in a fluid passes through the fixed volume defined by the intersecting laser beams. The LDV used in this investigation has four laser beams emitted by the probe, two in the horizontal direction and two in the vertical 


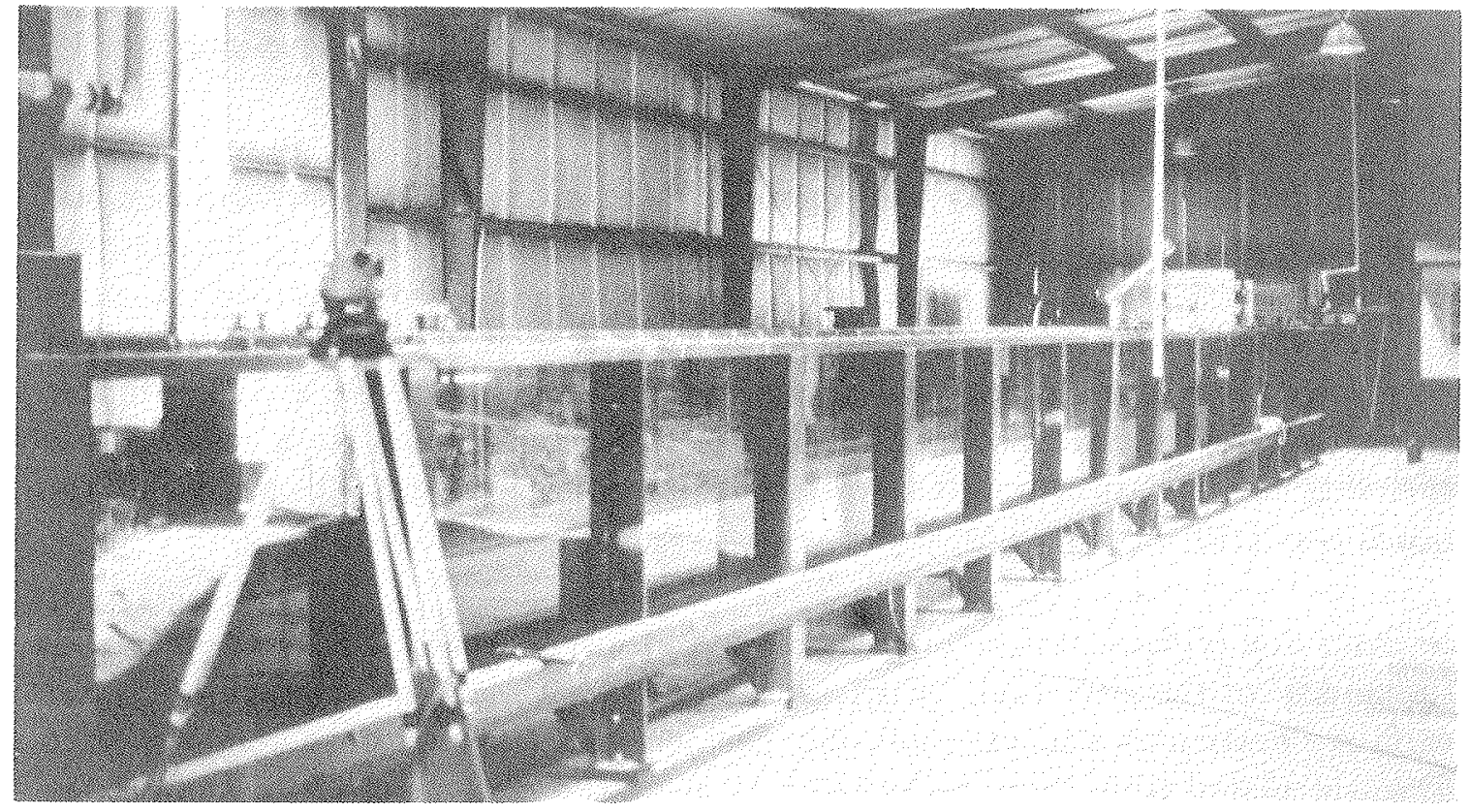

(a) Oblique View

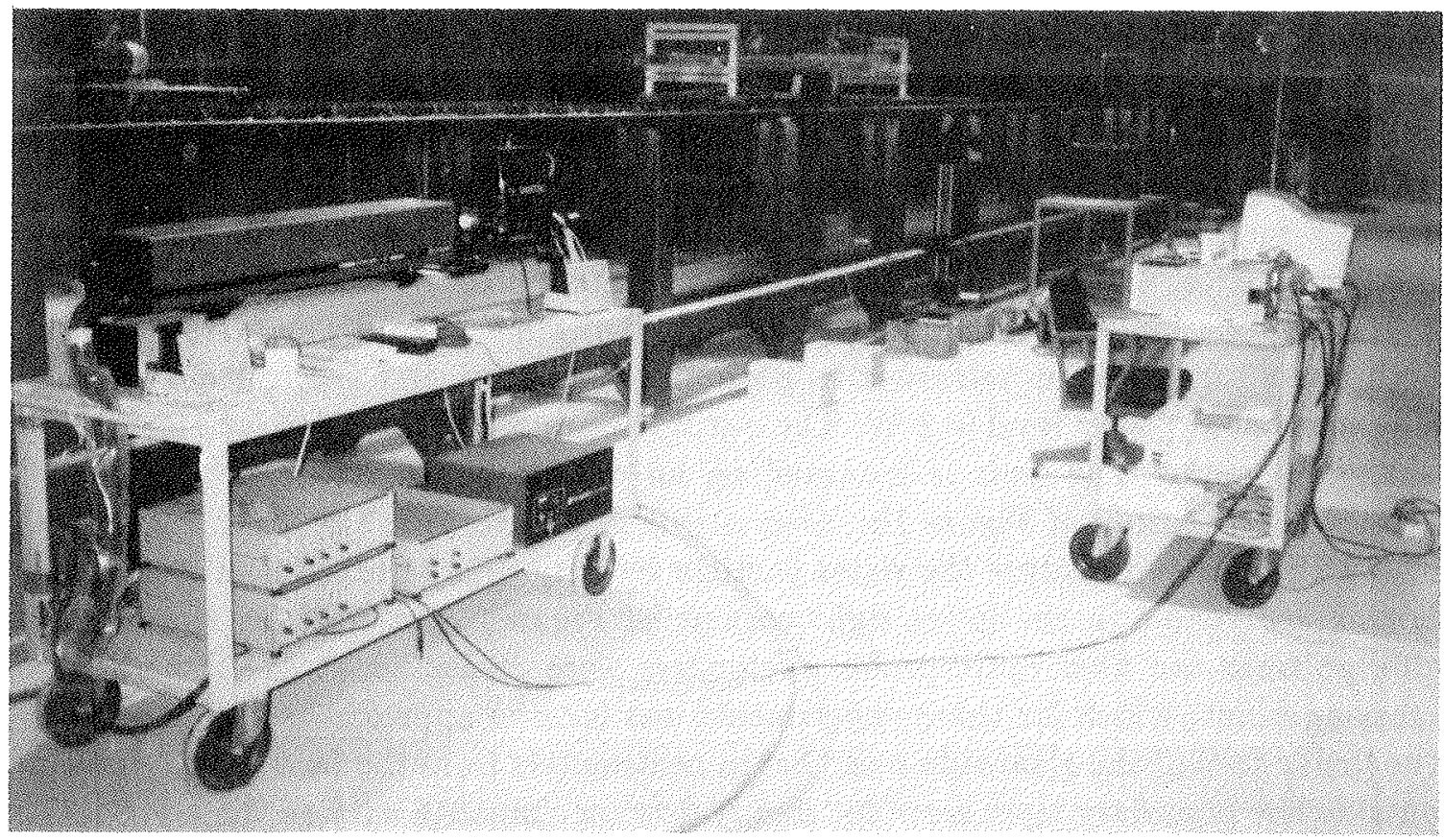

(b) Laser and Micro-computer

Figure 1: Wave Tank Photographs. (a) Oblique View; (b) Laser and Microcomputer 


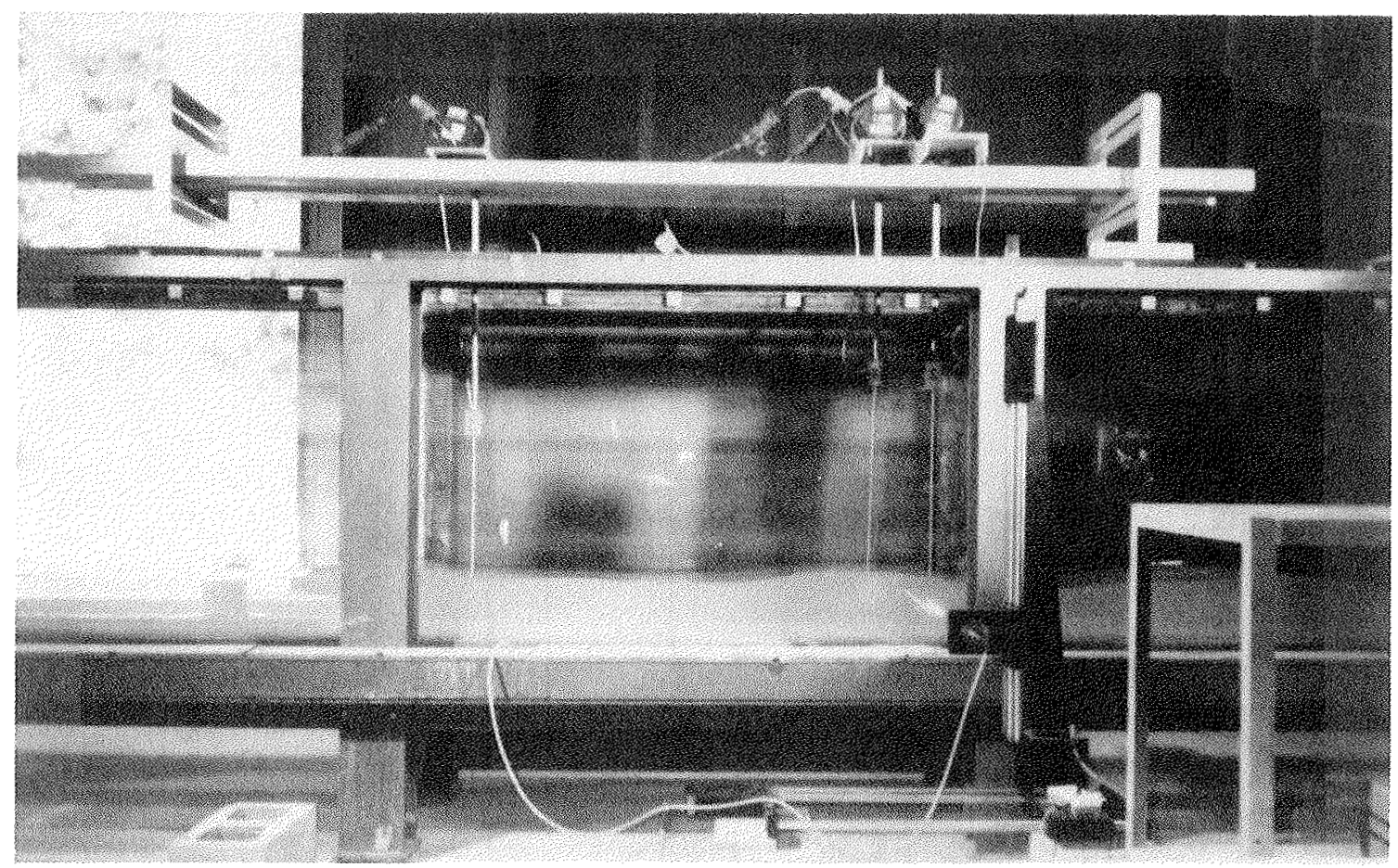

(a) Capacitance Type Wave Gages and Laser probe

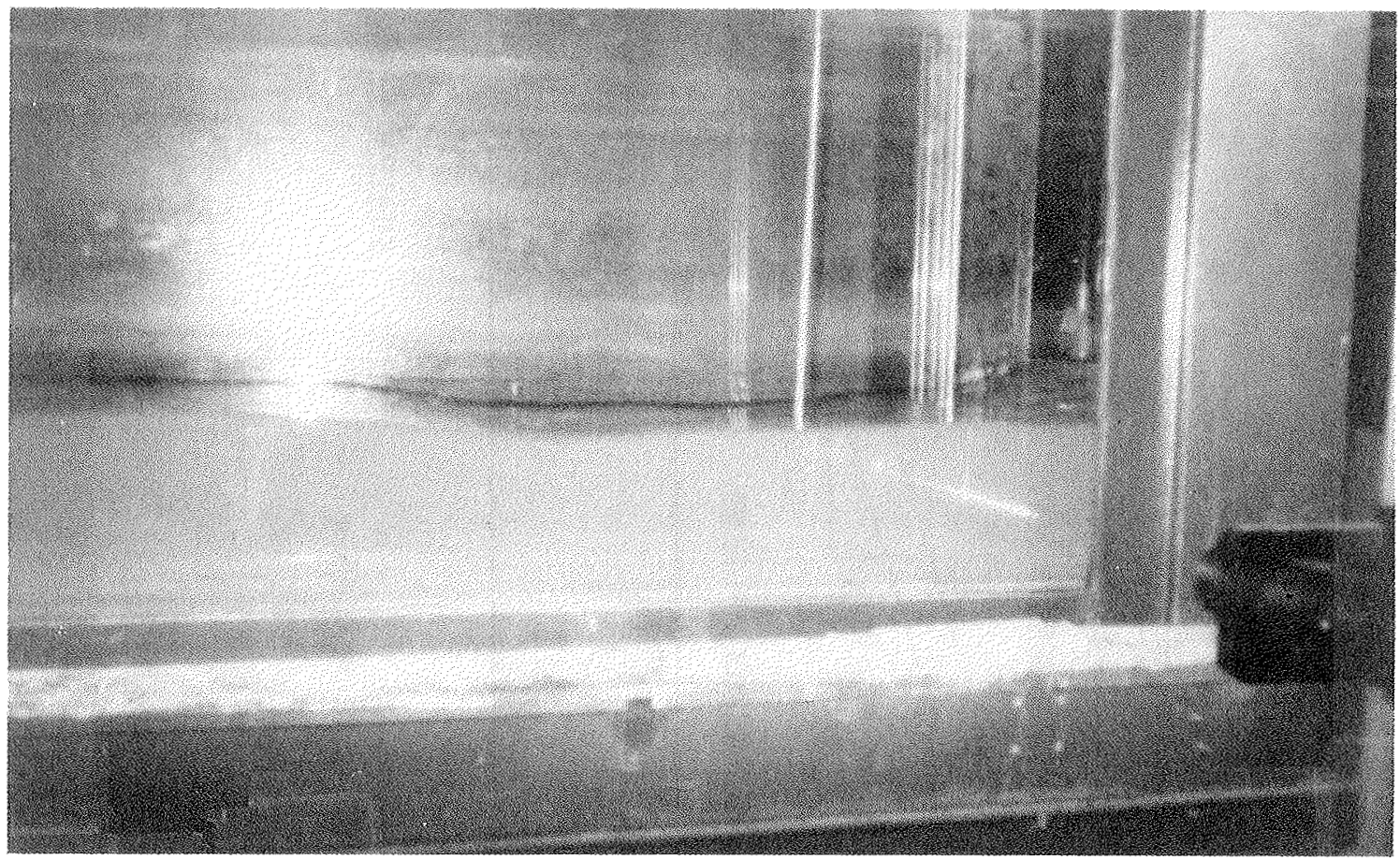

(b) Laser Probe

Figure 2: Equipment Close-up Photographs (a) Capacitance Type Wave Gages and Laser Probe (b) Laser Probe 


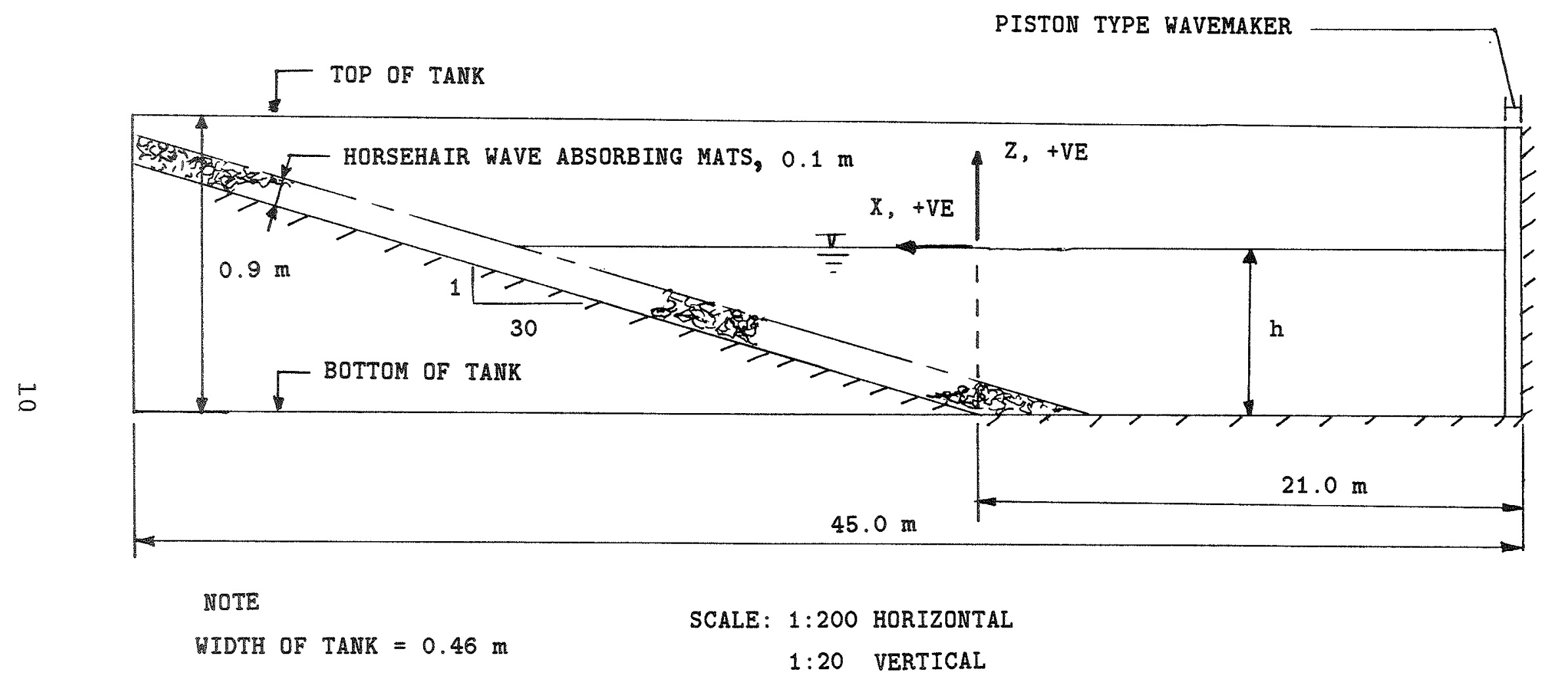

Figure 3: Wave Tank - Schematic 
direction. Each laser beam has a precise frequency and all the light waves are in phase. The intersection of the two beams, therefore, sets up an interference pattern of alternating light and dark bands called a "fringe" pattern. As a particle passes through this fringe pattern light is scattered when the particle is in a "light" band and is not scattered when it is in a dark band. The spacing of the fringe pattern is known from the physics of light. By measuring the frequency of the flashes of scattered light it is in principle possible to measure the velocity of the particle as it passes through the fringe pattern. By adjusting the relative phase of the two intersecting laser beams it is possible to get the fringe pattern itself moving at a fixed velocity thereby permitting differentiation between velocities in the positive and negative directions. The LDV used in this investigation detects the flashes of scattered light at the same probe that emits the laser beams. This is called measuring in the backscattering mode. The probe is connected to the laser and signal processing electronics by a fiber optic cable.

The doppler frequency range used was 33 to $333 \mathrm{khz}$, which provides an accuracy in velocity measurements of $\pm 0.015 \mathrm{~m} / \mathrm{s}$. In addition, the internal electronics have a constant offset that must be corrected. The velocity offsets are a constant source of error and are measured by placing the laser measuring point on the glass sidewalls of the wave tank. The velocity of the glass is certainly zero, and therefore, the velocity that the LDV measures can be applied as an offset to all future measurements. Typical offset values are shown in Figure 4a (Figure $4 \mathrm{a}$ is discussed later in this thesis). Offset values were determined before each days measurements.

Titanium dioxide particles from white latex paint were used to "seed" the wave tank in order to provide particles for scattering the light. The amount of seeding added to the tank was dictated by experience and a feel for the quality of the signal return. Too much seeding clouded the water excessively and attenuated the laser beam. Too little seeding resulted in "dropouts" whereby no particle passed through the laser intersection point resulting in no signal. About one teaspoon of white latex paint (without the binding agent) added to fresh tank water resulted in a good signal (approximately 1 teaspoon per $5 \mathrm{~m}^{3}$ ). Chlorine was also added to the tank to help control algae growth.

The measurements of velocities with the LDV and of wave heights with the capacitance type gauges were not synoptic. That is, a water particle velocity measurement at a certain instant in time cannot be matched with a water surface elevation measurement for the same instant in time. LDV velocities were recorded and analyzed on a CompuAdd $386 / 20 \mathrm{MHz}$ microcomputer while water surface elevations were measured and analyzed on a completely separate VAX minicomputer. Analysis of the calculated statistics for each time series was done mainly with the aid of a personal computer based spreadsheet. That is, statistics calculated from the LDV measurements and from the separate wave gauge measurements were 
Trme Series Analy $>$ is of LDV Valocity Data contalned in P1la: LI19.PRN

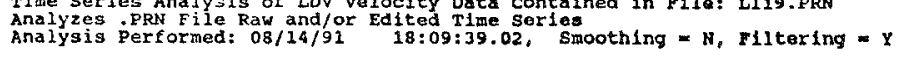
YEXT LISTING OF VELOCTTY DATA

Date: $07 / 03 / 91$

Number of Samples/channe1: 8192
Number of Channels Used: 2 of
sampling Frequency Used: $60 \mathrm{~Hz}$

OUTPUT OPTIONS

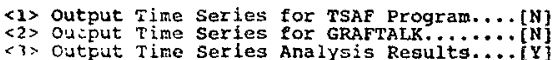

TIME SERIES EDITING OPTIONS

$<4>$ Simple point-to-point Filtaring........ [N]
$<5>$ Band pass Filtering.................. Filter Band
$[0.2000-5.000]$ sec TIME SERIES A:ALYYSIS OPTIONS

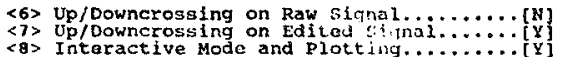

Output Files were:

Input LDV File: LII19.PRN

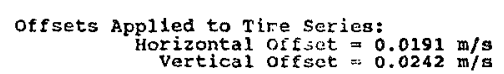

TIME SERIES STATISTYCS AND HISTOGRAMS - EDITED DATA:

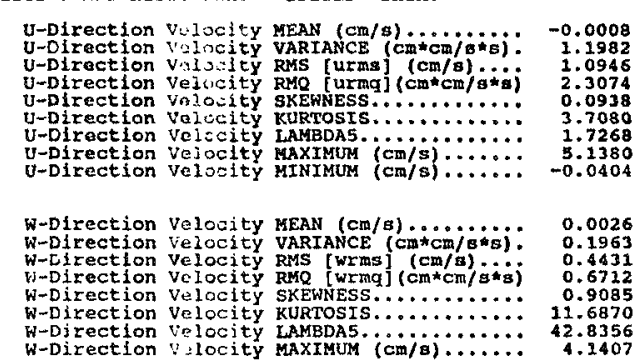

(a)
W-Direction Velocity MINIMUM $(\mathrm{cm} / \mathrm{s}) \ldots \ldots,-0.0292$

Positive U-Direction NUMBER OF PEAKS...
positive U-Direction Peaks UMEAN

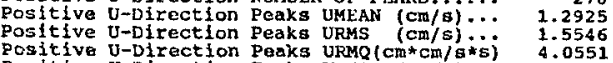

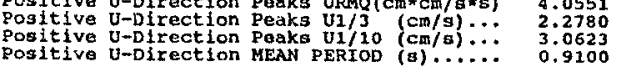

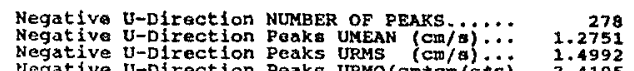

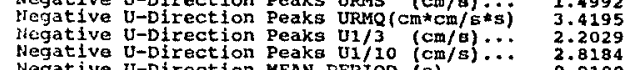
U-DIRECTION HISTOGRAM PEAKS
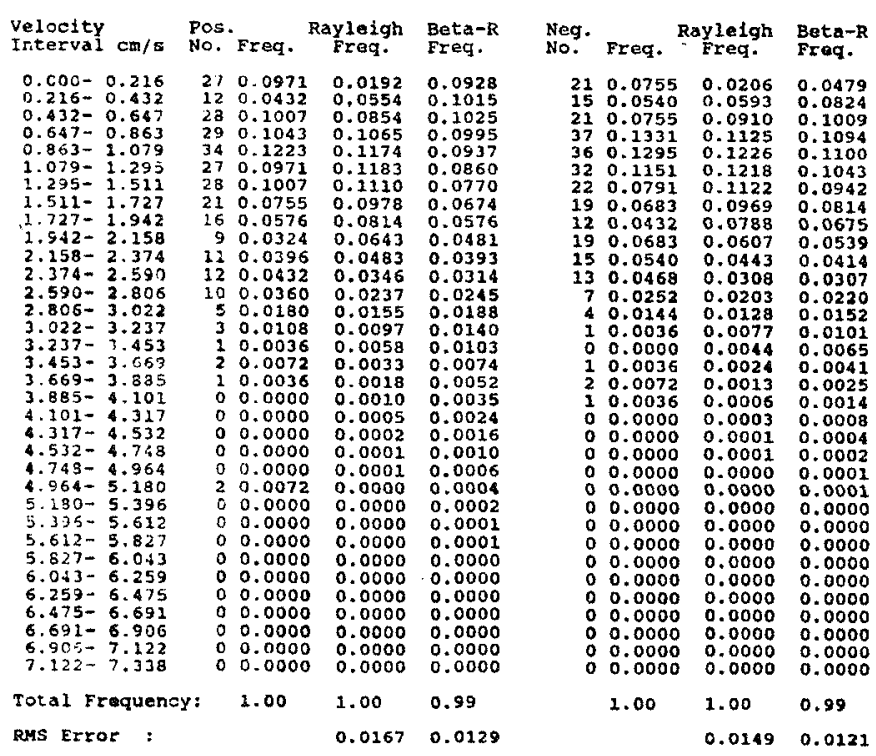

(b)

Figure 4: Computer Output - Water Particle Velocity Time Series Analysis 
TMS E:ror-i:

$\begin{array}{lll}0.5685 & 0.4387\end{array}$

Water depth $\begin{aligned} & 5.000 \mathrm{~cm} \\ & \mathrm{Umax}=221.47 \mathrm{~cm} / \mathrm{sec}\end{aligned}$

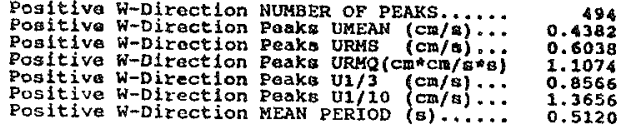

Negat Ive W-Direction NUMBER OF PEAKS.
Negative W-DIrection Peaks UMEAN (cmis)

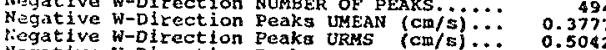

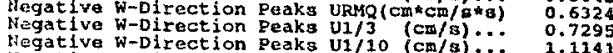

W-DIRECTION HISTOCRIAH, PEAKS

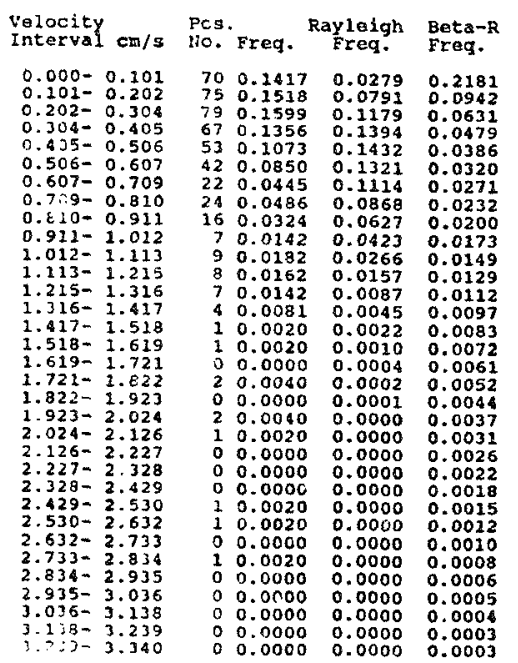

(c)
Freg.

$\begin{array}{llll}90 & 0.1822 & 0.0399 & 0.2510 \\ 76 & 0.1538 & 0.0120 & 0.2258 \\ 72 & 0.1457 & 0.156 & 0.258 \\ 72 & 0.157 & 0.1562 & 0.0891\end{array}$

$\begin{array}{llll}72 & \mathbf{0 . 1 4 5 7} & 0.1566 & 0.0891 \\ 777 & 0.1559 & 0.1722 & 0.0691\end{array}$

$\begin{array}{llll}54 & 0.1093 & 0.2604 & 0.0557 \\ 38 & 0.0759 & 0.1310 & 0.0455 \\ 29 & 0.0587 & 0.0955 & 0.035\end{array}$

$190.0385 \quad 0.062$

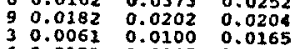

$\begin{array}{llll}6 & 0.0121 & 0.0045 & 0.0131 \\ 4 & 0.0081 & 0.0019 & 0.013\end{array}$

$\begin{array}{llll}2 & 0.0000 & 0.0007 \\ 3 & 0.0063 & 0.0002 \\ 0 & 0.000000 & 0.0001\end{array}$

$\begin{array}{lll}2 & 0.0061 & 0.000 \\ 0 & 0.0060 & 0.000 \\ 0 & 0.0000 & 0.0000\end{array}$

0.0000
00.0000
0 0.0000

$\begin{array}{lll}0.0000 & 0.0000 & 0.00000 \\ 0.0000 & 0.0007\end{array}$

$\begin{array}{ll}0 & 0.0000 \\ 0 & 0.0000 \\ 1 & 0.0020\end{array}$

$\begin{array}{ll}0.0020 & 0.0000 \\ 0.00000 & 0.0000\end{array}$

$0.0000 \quad 0.0000 \quad 0.0001$

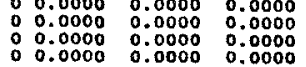

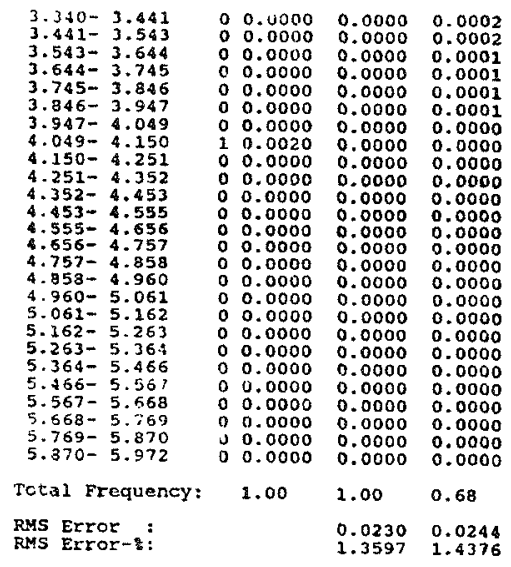

$\begin{array}{llll}0 & 0.0000 & 0.0000 & 0.0000 \\ 0 & 0.0000 & 0.0000 & 0.0000\end{array}$ $\begin{array}{llll}0 & 0.0000 & 0.0000 & 0.0000 \\ 0 & 0.0000 & 0.0000 & 0.0000 \\ 0 & 0.0000 & 0.0000 & 0.0000\end{array}$

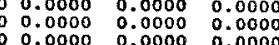
$\begin{array}{llll} & 0.0000 & 0.0000 & 0.0000 \\ 0 & 0.00000 & 0.0000 & 0.0000 \\ 0 & 0.00000000000 & 0.0000\end{array}$ $\begin{array}{llll}0 & 0.0000 & 0.0000 & 0.0000 \\ 0 & 0.0000 & 0.0000 & 0.0000 \\ 0 & 0.0000 & 0.00000 & 0.0000 \\ 0 & 0.0000 & 0.0000 & 0.0000\end{array}$ $\begin{array}{llll}0 & 0.0000 & 0.0000 & 0.0000 \\ 0 & 0.0000 & 0.0000 & 0.0000 \\ 0 & 0.0000 & 0.0000 & 0.0000 \\ 0 & 0.0000 & 0.0000 & 0.0000\end{array}$

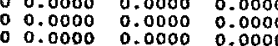

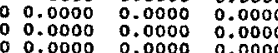

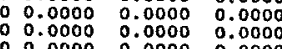
$\begin{array}{llll}0 & 0.0000 & 0.0000 & 0.0000 \\ 0 & 0.0000 & 0.0000 & 0.0000 \\ 0 & 0.0000 & 0.0000 & 0.0000\end{array}$

$\begin{array}{lll}1.00 & 1.00 \quad 0.82\end{array}$ $\begin{array}{lll}0.0228 & 0.9198 \\ 1.3042 & 0.1121\end{array}$

$\begin{gathered}\text { depth } \\ \text { w.max }\end{gathered}=\begin{gathered}50.00 \mathrm{~cm} \\ 36.82 \mathrm{~cm} / \mathrm{sec}\end{gathered}$

VELOCITY HISTCONMIS - EDTTED DATA

Horizontal velocity - u

NoTE: Velocitles are Non-Dimenslonalized
By the Standard Deviation of Entire Time Series

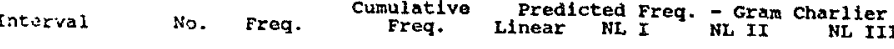

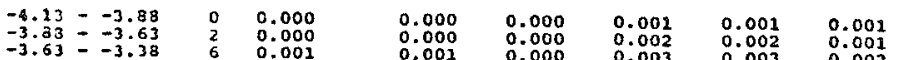

$-3.38=-3.13 \quad 6 \quad 0.001$

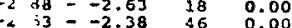

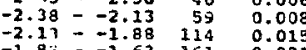

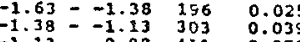

$-0.38=-0.63 \quad 604 \quad 0.07$

$\begin{array}{rrrr}-0.38 & -0.13 & 821 & 0.105 \\ -0.13 & -0.13 & 897 & 0.115 \\ 0.13 & 0.28 & 820 & 0.105 \\ 0.38 & 0.78 & 820 & 0.105\end{array}$

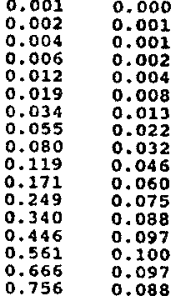

$\begin{array}{lll}0.002 & 0.001 & 0.001 \\ 0.002 & 0.002 & 0.001 \\ 0.003 & 0.003 & 0.002 \\ 0.004 & 0.004 & 0.002 \\ 0.006 & 0.006 & 0.004 \\ 0.007 & 0.007 & 0.006 \\ 0.007 & 0.007 & 0.007 \\ 0.005 & 0.007 & 0.008 \\ 0.003 & 0.005 & 0.008 \\ 0.0002 & 0.003 & 0.006 \\ 0.006 & 0.002 & 0.005 \\ 0.006 & 0.006 & 0.008 \\ 0.019 & 0.019 & 0.008 \\ 0.044 & 0.044 & 0.018 \\ 0.077 & 0.077 & 0.039 \\ 0.111 & 0.071 & 0.069 \\ 0.137 & 0.113 & 0.103 \\ 0.146 & 0.137 & 0.132 \\ 0.135 & 0.146 & 0.146 \\ 0.137 & 0.135 & 0.140 \\ 0.107 & 0.107 & 0.115\end{array}$

(d)

Figure 4: Continued 

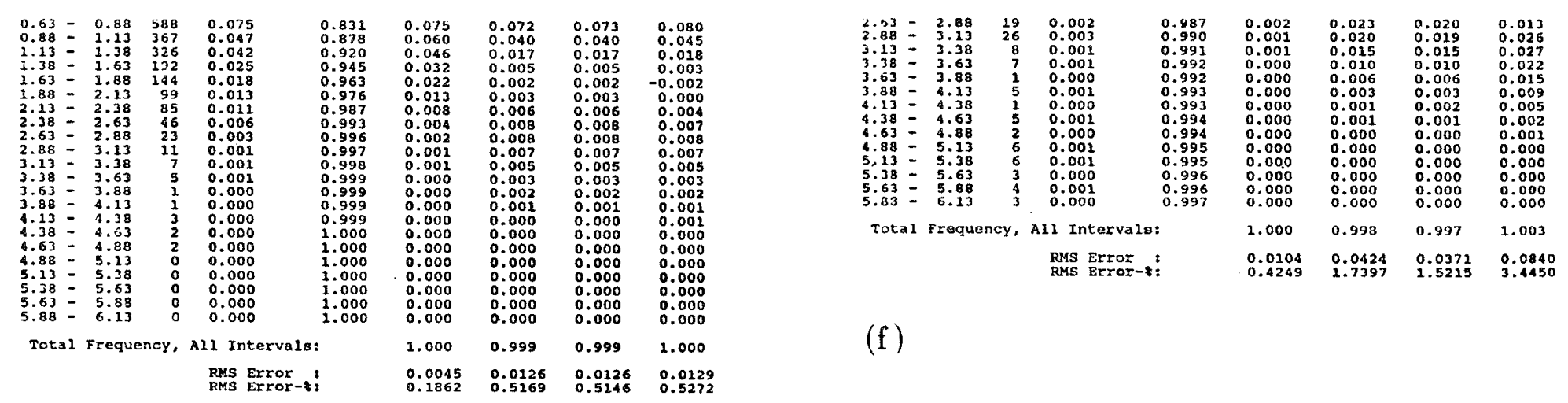

vertical velocity - "

NOTE: Velocitios are Non-Dimensionalized
By the Stzndard Deviation of Entire Time Serles

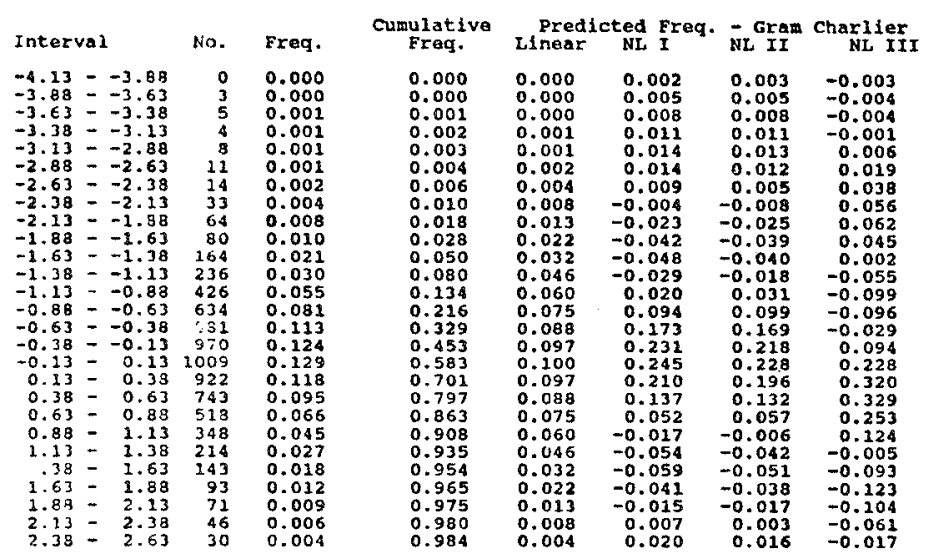

(e)

Figure 4: Continued 
Table 1: Experimental Plan

\begin{tabular}{|l|l|}
\hline \hline X-coordinate & $-10.5 \mathrm{~m}$ \\
Z-coordinate & mid-depth, near bottom \\
Water Depth & $50,30,20,15 \mathrm{~cm}$ \\
Peak Spectral Period & $1.0,1.5,2.0,2.5,3.0$ seconds \\
Significant Wave Height & $4.0,9.0 \mathrm{~cm}$ \\
\hline
\end{tabular}

entered by hand onto a spreadsheet. The spreadsheet was mainly used for calculating nondimensional parameters and tabulating data points. PC-based graphics software was used for graphing the results.

Three wave gauges were used to determine the incident and reflected wave height spectrums using the method of Goda and Suzuki (1976). The reflection coefficient is calculated by dividing the total energy in the reflected spectrum by the total energy in the incident spectrum. Figure 5 graphs the incident and reflected spectrums. The discrete spectral components graphed in Figure 5 are written to a separate data file for use in calculating $u_{r m s}$ and $w_{r m s}$.

\subsection{Experimental Plan}

At the core of the experimental plan are 80 time series distinguished by combining every way possible the values of the five variables listed in Table 1. Table 2 lists all the time series which were run for irregular waves.

Wave parameters were selected to cover a complete range of wave conditions consistent with the limitations of the wave tank. Of particular interest were nonlinear wave conditions, and the experimental program was structured so wave nonlinearities more or less gradually increased as the testing progressed. This thesis is intended to provide a basis for further studies related to sediment transport and scour. Therefore the measurement points are in all cases at mid-depth or near bottom. Table 2 also lists several time series that have identical wave conditions to time series run previously. These series were run to show repeatability and spatial homogeneity in the velocity field.

Not listed in Table 2 are the eighteen regular wave time series that were run before the irregular wave conditions. These regular waves were run in order to optimize the design of the wave absorbing spending beach. 


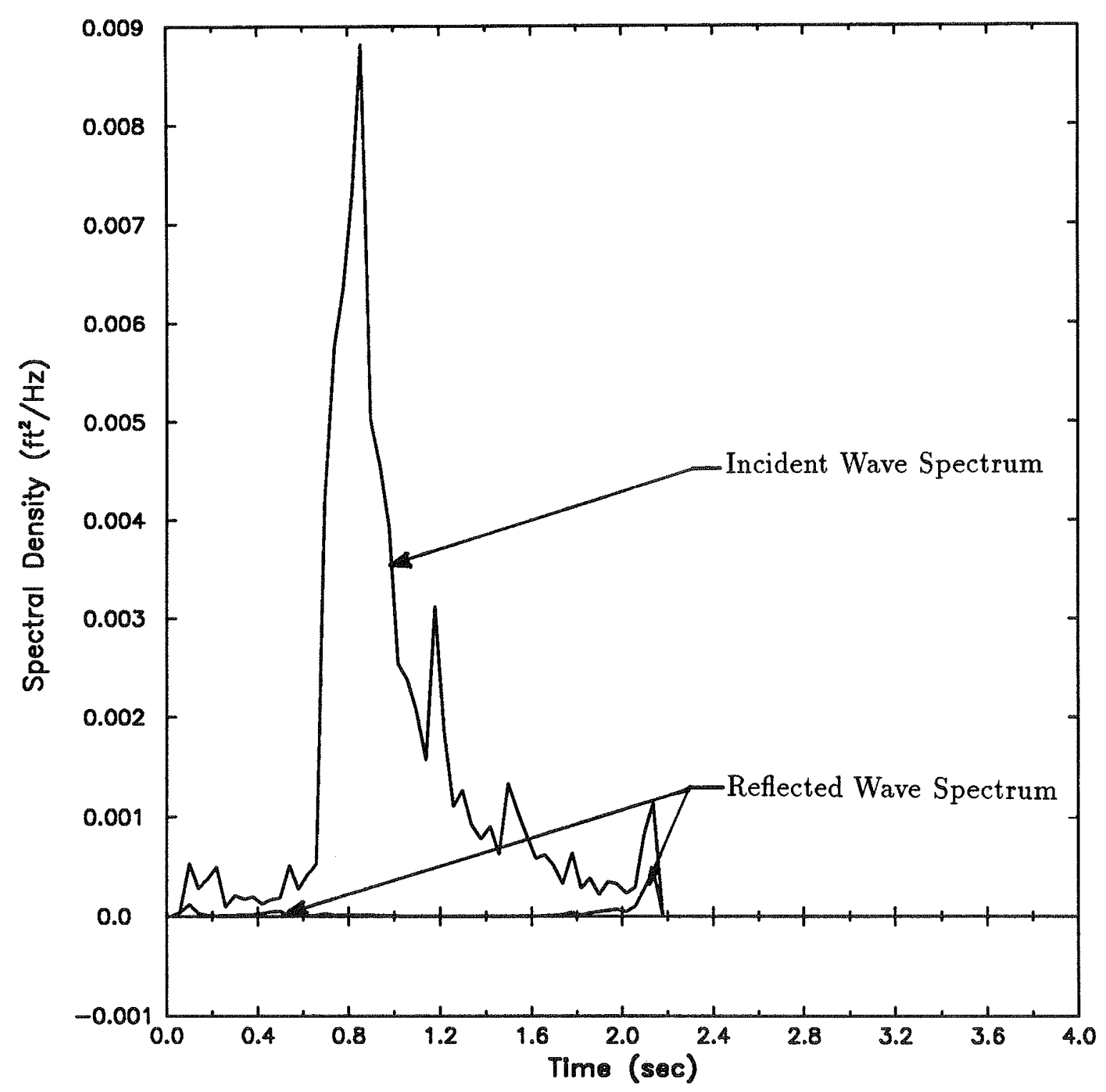

Figure 5: Measured Incident and Reflected Wave Spectra 
Table 2: Summary - Individual Time Series

\begin{tabular}{|c|c|c|c|c|c|c|}
\hline \multirow[b]{2}{*}{$\begin{array}{l}\text { Run } \\
\text { No. }\end{array}$} & \multicolumn{2}{|c|}{ Measuring Point } & \multicolumn{3}{|c|}{ Planned Conditions } & \multirow[b]{2}{*}{ Notes } \\
\hline & $\begin{array}{r}\mathrm{X} \text {-coord. } \\
(\mathrm{m})\end{array}$ & $\begin{array}{r}\text { Z-Coord. } \\
(\mathrm{cm})\end{array}$ & $\begin{array}{c}h \\
(\mathrm{~cm})\end{array}$ & $\begin{array}{c}T_{p} \\
(\mathrm{sec})\end{array}$ & $\begin{array}{l}H_{m o} \\
(\mathrm{~cm})\end{array}$ & \\
\hline$\overline{1}$ & -7.00 & -10.00 & 20.00 & 1.25 & 4.00 & \\
\hline 2 & -7.00 & -10.00 & 20.00 & 1.25 & 9.00 & \\
\hline 3 & -10.50 & -10.00 & 20.00 & 1.25 & 4.00 & \\
\hline 4 & -10.50 & -10.00 & 20.00 & 1.25 & 9.00 & \\
\hline 5 & -10.50 & -20.00 & 40.00 & 2.50 & 4.00 & \\
\hline 6 & -10.50 & -20.00 & 40.00 & 2.50 & 9.00 & \\
\hline 7 & -7.00 & -20.00 & 40.00 & 2.50 & 4.00 & \\
\hline 8 & -7.00 & -20.00 & 40.00 & 2.50 & 9.00 & \\
\hline 9 & -10.50 & -25.00 & 50.00 & 1.00 & 4.00 & \\
\hline 10 & -10.50 & -25.00 & 50.00 & 1.00 & 9.00 & \\
\hline 11 & -10.50 & -25.00 & 50.00 & 1.50 & 4.00 & \\
\hline 12 & -10.50 & -25.00 & 50.00 & 1.50 & 9.00 & \\
\hline 13 & -10.50 & -25.00 & 50.00 & 2.00 & 4.00 & \\
\hline 14 & -10.50 & -25.00 & 50.00 & 2.00 & 9.00 & \\
\hline 140 & -10.50 & -25.00 & 50.00 & 2.00 & 9.00 & repeat \\
\hline 141 & -10.50 & -25.00 & 50.00 & 2.00 & 9.00 & repeat \\
\hline 15 & -10.50 & -25.00 & 50.00 & 2.50 & 4.00 & \\
\hline 150 & -10.50 & -25.00 & 50.00 & 2.50 & 4.00 & repeat \\
\hline 151 & -7.00 & -25.00 & 50.00 & 2.50 & 4.00 & repeat \\
\hline 152 & -8.75 & -25.00 & 50.00 & 2.50 & 4.00 & repeat \\
\hline 153 & -10.50 & -25.00 & 50.00 & 2.50 & 4.00 & repeat \\
\hline 16 & -10.50 & -25.00 & 50.00 & 2.50 & 9.00 & \\
\hline 17 & -10.50 & -25.00 & 50.00 & 3.00 & 4.00 & \\
\hline 170 & -10.50 & -25.00 & 50.00 & 3.00 & 4.00 & repeat \\
\hline 18 & -10.50 & -25.00 & 50.00 & 3.00 & 9.00 & \\
\hline 19 & -10.50 & -47.00 & 50.00 & 1.00 & 4.00 & \\
\hline 20 & -10.50 & -47.00 & 50.00 & 1.00 & 9.00 & \\
\hline 21 & -10.50 & -47.00 & 50.00 & 1.50 & 4.00 & \\
\hline 22 & -10.50 & -47.00 & 50.00 & 1.50 & 9.00 & \\
\hline 23 & -10.50 & -47.00 & 50.00 & 2.00 & 4.00 & \\
\hline 24 & -10.50 & -47.00 & 50.00 & 2.00 & 9.00 & \\
\hline 25 & -10.50 & -47.00 & 50.00 & 2.50 & 4.00 & \\
\hline 26 & -10.50 & -47.00 & 50.00 & 2.50 & 9.00 & \\
\hline 27 & -10.50 & -47.00 & 50.00 & 3.00 & 4.00 & \\
\hline 28 & -10.50 & -47.00 & 50.00 & 3.00 & 9.00 & \\
\hline 29 & -10.50 & -15.00 & 50.00 & 1.00 & 4.00 & \\
\hline
\end{tabular}


Table 2: Continued

\begin{tabular}{|r|rr|ccc|l|}
\hline \hline & \multicolumn{2}{|c|}{ Measuring Point } & \multicolumn{3}{|c|}{ Planned Conditions } & \\
\cline { 2 - 6 } Run & X-coord. & Z-Coord. & $h$ & $T_{p}$ & $H_{\text {mo }}$ & \\
No. & $(\mathrm{m})$ & $(\mathrm{cm})$ & $(\mathrm{cm})$ & $(\mathrm{sec})$ & $(\mathrm{cm})$ & Notes \\
\hline 30 & -10.50 & -15.00 & 30.00 & 1.00 & 9.00 & \\
31 & -10.50 & -15.00 & 30.00 & 1.50 & 4.00 & \\
32 & -10.50 & -15.00 & 30.00 & 1.50 & 9.00 & \\
33 & -10.50 & -15.00 & 30.00 & 2.00 & 4.00 & \\
34 & -10.50 & -15.00 & 30.00 & 2.00 & 9.00 & \\
340 & -7.00 & -15.00 & 30.00 & 2.00 & 9.00 & repeat \\
341 & -8.75 & -15.00 & 30.00 & 2.00 & 9.00 & repeat \\
35 & -10.50 & -15.00 & 30.00 & 2.50 & 4.00 & \\
36 & -10.50 & -15.00 & 30.00 & 2.50 & 9.00 & \\
37 & -10.50 & -15.00 & 30.00 & 3.00 & 4.00 & \\
38 & -10.50 & -15.00 & 30.00 & 3.00 & 9.00 & \\
39 & -10.50 & -27.00 & 30.00 & 1.00 & 4.00 & \\
40 & -10.50 & -27.00 & 30.00 & 1.00 & 9.00 & \\
41 & -10.50 & -27.00 & 30.00 & 1.50 & 4.00 & \\
42 & -10.50 & -27.00 & 30.00 & 1.50 & 9.00 & \\
43 & -10.50 & -27.00 & 30.00 & 2.00 & 4.00 & \\
44 & -10.50 & -27.00 & 30.00 & 2.00 & 9.00 & \\
45 & -10.50 & -27.00 & 30.00 & 2.50 & 4.00 & \\
46 & -10.50 & -27.00 & 30.00 & 2.50 & 9.00 & \\
47 & -10.50 & -27.00 & 30.00 & 3.00 & 4.00 & \\
48 & -10.50 & -27.00 & 30.00 & 3.00 & 9.00 & \\
49 & -10.50 & -10.00 & 20.00 & 1.00 & 4.00 & \\
50 & -10.50 & -10.00 & 20.00 & 1.00 & 9.00 & \\
51 & -10.50 & -10.00 & 20.00 & 1.50 & 4.00 & \\
52 & -10.50 & -10.00 & 20.00 & 1.50 & 9.00 & \\
53 & -10.50 & -10.00 & 20.00 & 2.00 & 4.00 & \\
54 & -10.50 & -10.00 & 20.00 & 2.00 & 9.00 & \\
55 & -10.50 & -10.00 & 20.00 & 2.50 & 4.00 & \\
56 & -10.50 & -10.00 & 20.00 & 2.50 & 9.00 & \\
57 & -10.50 & -10.00 & 20.00 & 3.00 & 4.00 & \\
58 & -10.50 & -10.00 & 20.00 & 3.00 & 9.00 & \\
580 & -10.50 & -10.00 & 20.00 & 3.00 & 9.00 & repeat \\
581 & -10.50 & -10.00 & 20.00 & 3.00 & 9.00 & repeat \\
582 & -8.75 & -10.00 & 20.00 & 3.00 & 9.00 & repeat \\
583 & -7.00 & -10.00 & 20.00 & 3.00 & 9.00 & repeat \\
59 & -10.50 & -17.00 & 20.00 & 1.00 & 4.00 & \\
\hline \hline & & & & & & \\
\hline
\end{tabular}


Table 2: Continued

\begin{tabular}{|c|c|c|c|c|c|c|}
\hline \multirow[b]{2}{*}{$\begin{array}{c}\text { Run } \\
\text { No. }\end{array}$} & \multicolumn{2}{|c|}{ Measuring Point } & \multicolumn{3}{|c|}{ Planned Conditions } & \multirow[b]{2}{*}{ Notes } \\
\hline & $\begin{array}{r}\mathrm{X} \text {-coord. } \\
(\mathrm{m})\end{array}$ & $\begin{array}{r}\text { Z-coord. } \\
(\mathrm{cm})\end{array}$ & $\begin{array}{c}h \\
(\mathrm{~cm})\end{array}$ & $\begin{array}{c}T_{p} \\
(\mathrm{sec})\end{array}$ & $\begin{array}{l}H_{m o} \\
(\mathrm{~cm})\end{array}$ & \\
\hline 60 & -10.50 & -17.00 & 20.00 & 1.00 & 9.00 & \\
\hline 61 & -10.50 & -17.00 & 20.00 & 1.50 & 4.00 & \\
\hline 62 & -10.50 & -17.00 & 20.00 & 1.50 & 9.00 & \\
\hline 63 & -10.50 & -17.00 & 20.00 & 2.00 & 4.00 & \\
\hline 64 & -10.50 & -17.00 & 20.00 & 2.00 & 9.00 & \\
\hline 65 & -10.50 & -17.00 & 20.00 & 2.50 & 4.00 & \\
\hline 66 & -10.50 & -17.00 & 20.00 & 2.50 & 9.00 & \\
\hline 67 & -10.50 & -17.00 & 20.00 & 3.00 & 4.00 & \\
\hline 68 & -10.50 & -17.00 & 20.00 & 3.00 & 9.00 & \\
\hline 69 & -10.50 & -7.50 & 15.00 & 1.00 & 4.00 & \\
\hline 70 & -10.50 & -7.50 & 15.00 & 1.00 & 9.00 & \\
\hline 71 & -10.50 & -7.50 & 15.00 & 1.50 & 4.00 & \\
\hline 710 & -10.50 & -7.50 & 15.00 & 1.50 & 4.00 & repeat \\
\hline 711 & -10.50 & -7.50 & 15.00 & 1.50 & 4.00 & repeat \\
\hline 712 & -10.50 & -7.50 & 15.00 & 1.50 & 4.00 & repeat \\
\hline 713 & -8.75 & -7.50 & 15.00 & 1.50 & 4.00 & repeat \\
\hline 714 & -7.00 & -7.50 & 15.00 & 1.50 & 4.00 & repeat \\
\hline 72 & -10.50 & -7.50 & 15.00 & 1.50 & 9.00 & \\
\hline 73 & -10.50 & -7.50 & 15.00 & 2.00 & 4.00 & \\
\hline 74 & -10.50 & -7.50 & 15.00 & 2.00 & 9.00 & \\
\hline 75 & -10.50 & -7.50 & 15.00 & 2.50 & 4.00 & \\
\hline 76 & -10.50 & -7.50 & 15.00 & 2.50 & 9.00 & \\
\hline 77 & -10.50 & -7.50 & 15.00 & 3.00 & 4.00 & \\
\hline 78 & -10.50 & -7.50 & 15.00 & 3.00 & 9.00 & \\
\hline 79 & -10.50 & -12.00 & 15.00 & 1.00 & 4.00 & \\
\hline 80 & -10.50 & -12.00 & 15.00 & 1.00 & 9.00 & \\
\hline 81 & -10.50 & -12.00 & 15.00 & 1.50 & 4.00 & \\
\hline 82 & -10.50 & -12.00 & 15.00 & 1.50 & 9.00 & \\
\hline 83 & -10.50 & -12.00 & 15.00 & 2.00 & 4.00 & \\
\hline 84 & -10.50 & -12.00 & 15.00 & 2.00 & 9.00 & \\
\hline 85 & -10.50 & -12.00 & 15.00 & 2.50 & 4.00 & \\
\hline 86 & -10.50 & -12.00 & 15.00 & 2.50 & 9.00 & \\
\hline 87 & -10.50 & -12.00 & 15.00 & 3.00 & 4.00 & \\
\hline 88 & -10.50 & -12.00 & 15.00 & 3.00 & 9.00 & \\
\hline 880 & -10.50 & -12.00 & 15.00 & 3.00 & 9.00 & \\
\hline
\end{tabular}


Each irregular wave time series was run in the wave tank for approximately 5 min. Collection of velocity data with the LDV began $20 \mathrm{~s}$ after the start of wave generation to allow time for the first waves to travel down the tank and be reflected back past the wave gauge array. Velocity data were collected at a $30 \mathrm{~Hz}$ rate for each velocity component for $273 \mathrm{~s}$. Collection of water elevation data with the wave gauges was at a $20 \mathrm{H}$ rate for $250 \mathrm{~s}$. The 5 min. length of each time series was selected based on experience. A long time series is desired for ensuring stable statistics. However, a short time series minimizes the buildup of reflected waves.

\subsection{Computer Output}

Figures 4 and 5 are hardcopy outputs for one time series. Figure 4 is for velocity data measured by the LDV. Figure 5 is from the VAX and is the result of the analysis of water surface elevations (tabulated statistics from the VAX computer are not given). The calculated statistics from each of these two outputs were then loaded on a spreadsheet for further study of trends between different time series. (In Figure 4a, a value of $-0.0404 \mathrm{~cm} / \mathrm{s}$ for the "U-direction velocity minimum" refers to the smallest absolute value velocity measured in the time series.)

Figure 6 shows a portion of one measured velocity time series produced by the LDV and subjected to various filtering options. Similar graphs were produced for every time series during the experiments and examined visually on the computer monitor. The plots provide a useful check on the quality of the velocity time series. Evident in the raw data (Figure 6a) are high frequency oscillations caused by turbulence and imperfections in the electronic data acquisition systems. The effect of filtering the data is clearly seen in Figures $6 \mathrm{~b}$ to $6 \mathrm{~d}$. Filtering is discusssed later in this thesis. Figure 6a is not typical of all the time series. Approximately half the runs looked like Figure $6 \mathrm{~b}$ prior to any filtering. Based on a visual examination of portions of all the velocity time series, the author is confident that the velocity measurements were of a very high quality.

\subsection{Wave Signal Generation}

Waves were created in the tank by a vertical board moved through the water by a computer controlled hydraulic ram. The exact horizontal position of the board was updated every $1 / 60$ of a second. The board signal was generated by a VAX Fortran program called TSGMN3P1.FOR. The user enters into the program the following parameters: 


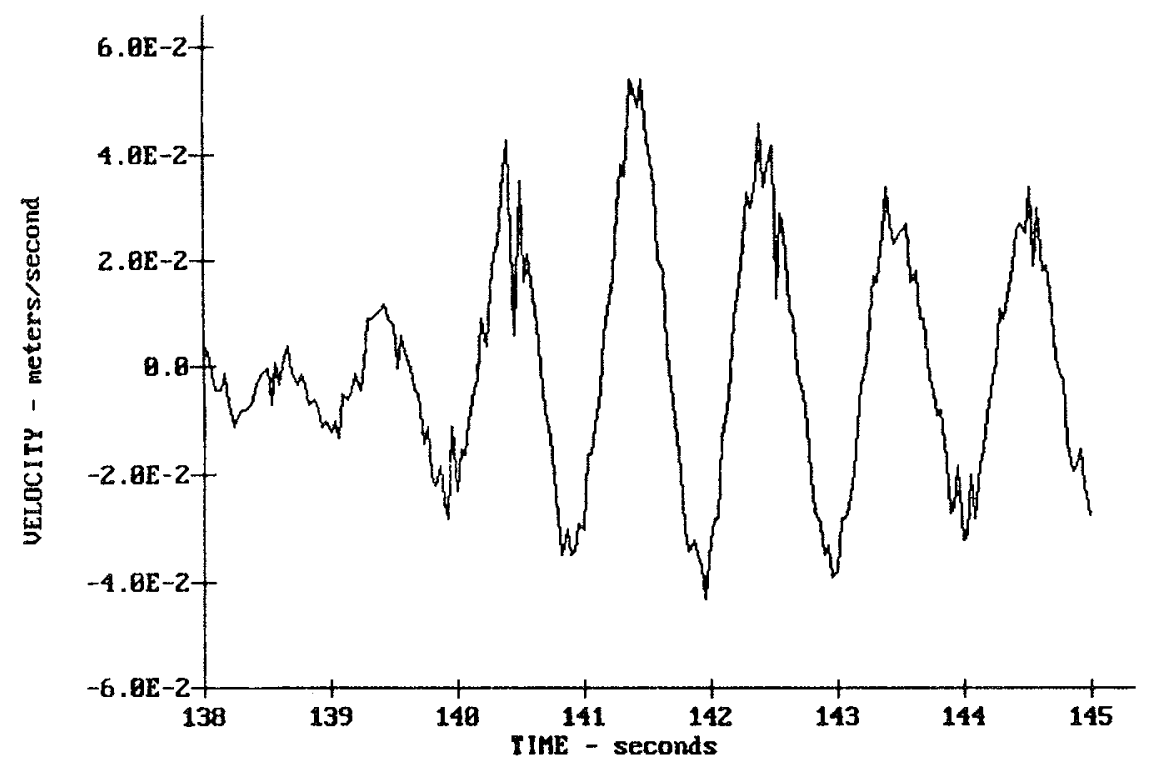

(a) Raw Data

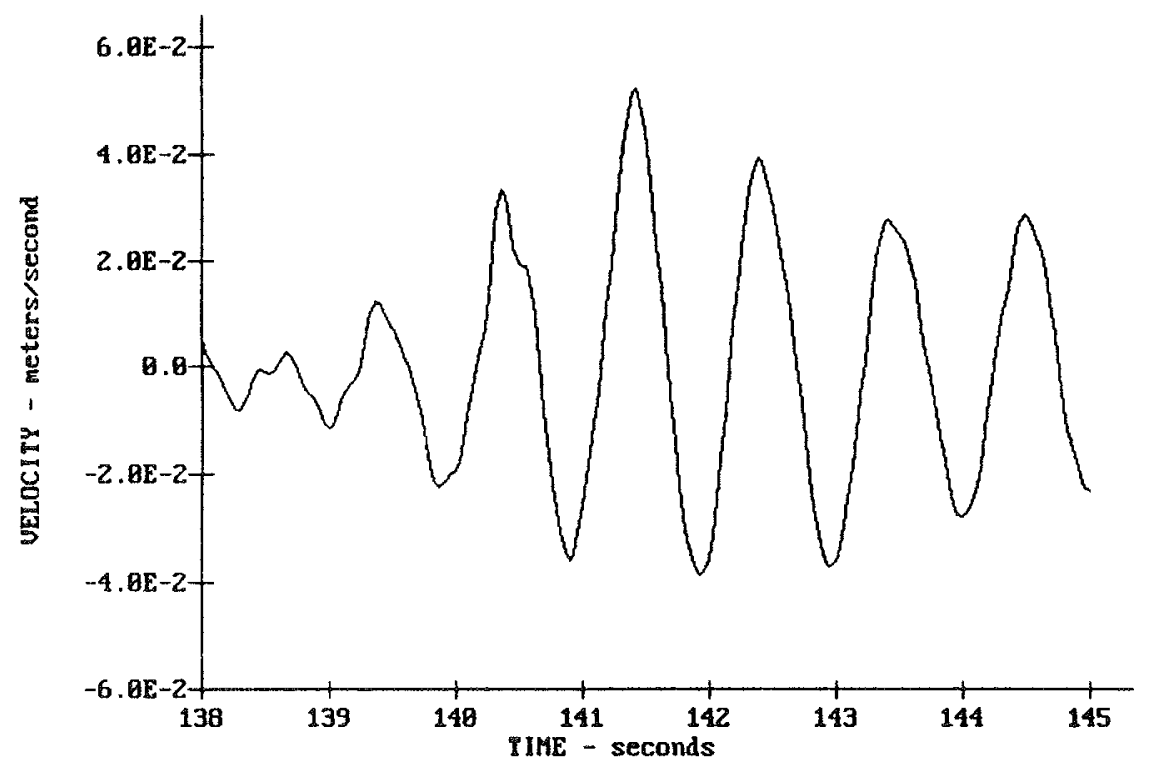

(b) Filtered Data $[0.20-5.00] \mathrm{s}$

Figure 6: Velocity Time Series - Run No. 19. (a) Raw Data; (b) Filtered Data [0.20-5.00] s; (c) Filtered Data [0.20-10.00] s; (d) Filtered Data [0.10-5.00] s 


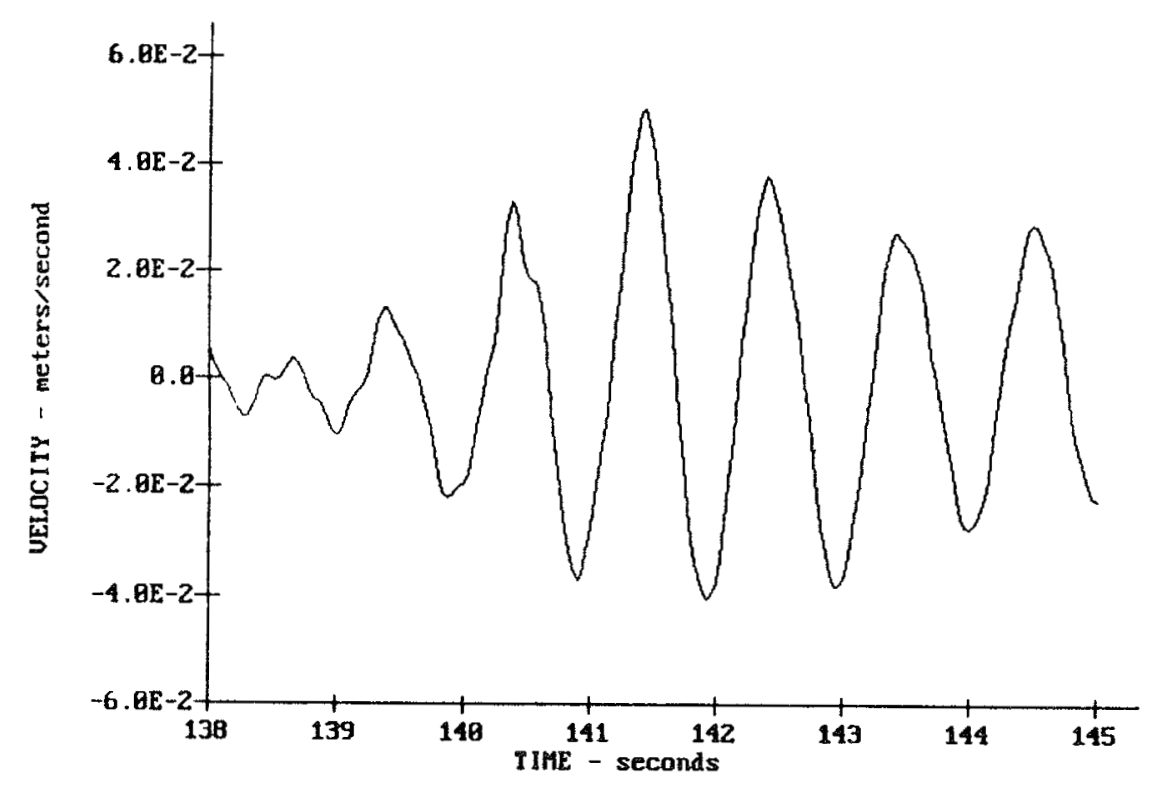

(c) Filtered Data [0.20-10.00] s

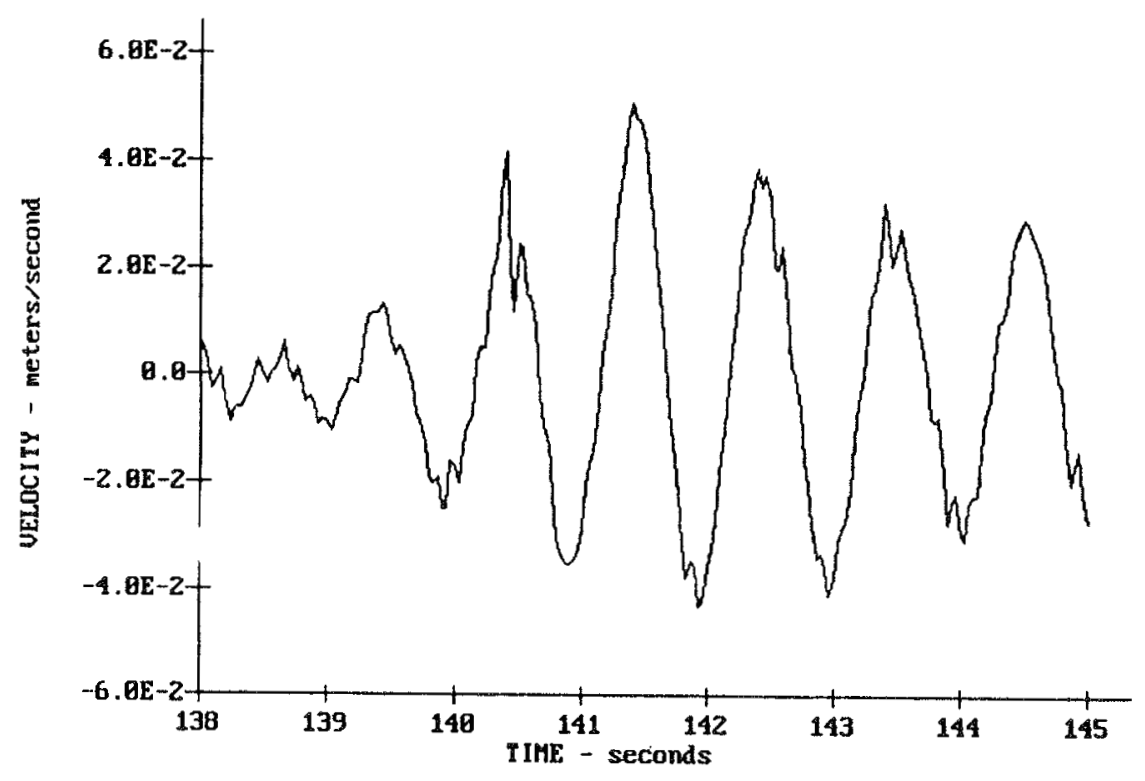

(d) Filtered Data [0.10-5.00] s

Figure 6: Continued 
- Water Depth

- Peak Spectral Period

- Significant Wave Height

- Spectral Shape Parameters (controls whether spectrum is a JONSWAP, Single Peaked TMA or several other shapes)

- Length of Time Series

- Seed number for randomization of linear wave superposition

The program creates a spectrum of waves based upon linear superposition of 128 components or spectral lines. Each component represents a certain wave height for a certain frequency. The phase relationship between different linear components is random. The theory relating the wave mechanics to the board motion is based on linear wave theory (Dean and Dalrymple 1984).

The TMA spectral shape (Bouws, et al. 1985, Hughes, 1984) is a depth dependent form of the JONSWAP spectral shape. The JONSWAP spectrum is based on empirical studies of wind wave spectra in the North Sea. The TMA spectrum uses over 2800 wind wave spectra at various depths and locations to empirically define the JONSWAP parameters for wind seas that have reached growth equilibrium in shallow water. The TMA spectrum is single peaked. 


\section{THE ROOT-MEAN-SQUARE VELOCITY}

\subsection{Theory}

The root-mean-square water particle velocity under irregular waves has been derived for horizontal velocities by Hughes (1992). The outline of his derivation is reproduced below.

The sea surface elevation, $\eta(x, t)$, of a linear, partially reflected, unidirectional wave on a flat bottom can be expressed as (Dean and Dalrymple 1984)

$$
\eta(x, t)=a \cos (k x-\sigma t)+a K_{r} \cos (k x+\sigma t+\theta)
$$

where

$a=$ amplitude of the incident wave,

$k=$ wave number, $(=2 \pi / L$, where $L$ is wave length $)$

$x=$ horizontal position,

$\sigma=$ angular wave frequency $(=2 \pi / T$, where $T$ is wave period $)$,

$t=$ time,

$K_{r}=$ reflection coefficient $\left(=a_{\text {incident }} / a_{\text {reflected }}\right)$, and

$\theta=$ phase shift due to reflection.

The first term in Equation 1 represents the incident wave, and the second term the partially reflected wave. For convenience the initial phase angle of the incident component is taken as zero at $t=0$ and $x=0$.

The fluid particle velocities under such a water surface can be easily derived from velocity potential theory as

$$
\begin{aligned}
& u(x, z, t)=\frac{a g k}{\sigma} \frac{\cosh k(h+z)}{\cosh k h}\left[K_{r} \cos (k x-\sigma t+\theta)-\cos (k x+\sigma t)\right] \\
& w(x, z, t)=\frac{a g k}{\sigma} \frac{\sinh k(h+z)}{\cosh k h}\left[K_{r} \sin (k x-\sigma t+\theta)-\sin (k x+\sigma t)\right]
\end{aligned}
$$

where

$u=$ horizontal component of velocity,

$w=$ vertical component of velocity,

$g=$ gravitational acceleration,

$h=$ water depth, and 
$z=$ vertical coordinate, positive upward, with $z=0$ at the still water level, and $z=-h$ at the bottom.

By linearly superimposing many components and making use of the autocorrelation function, it is possible to derive expressions for $u_{r m s}$ and $w_{r m s}$, the rootmean-squared (RMS) velocity (Hughes 1992). (In this thesis, lower case letters $u$ and $w$ represent statistics related to the entire time series. Upper case letters $U$ and $W$ represent statistics related to the peak velocities, for example $U_{r m s}$ and $W_{\text {mean }}$.)

$$
\begin{aligned}
& u_{r m s}^{2}=\sum_{n=1}^{\infty}\left(\frac{g k_{n}}{\sigma_{n}}\right)^{2} \frac{\cosh ^{2} k_{n}(h+z)}{\cosh ^{2} k_{n} h}\left(1-2 K_{r_{n}} \cos \left(2 k_{n} x+\theta_{n}\right)+K_{r_{n}}^{2}\right) \frac{a_{n}^{2}}{2} \\
& w_{r m s}^{2}=\sum_{n=1}^{\infty}\left(\frac{g k_{n}}{\sigma_{n}}\right)^{2} \frac{\sinh ^{2} k_{n}(h+z)}{\cosh ^{2} k_{n} h}\left(1+2 K_{r_{n}} \cos \left(2 k_{n} x+\theta_{n}\right)+K_{r_{n}}^{2}\right) \frac{a_{n}^{2}}{2}
\end{aligned}
$$

The rms velocity is a useful and important parameter in studies of turbulence and sediment transport. The parameters $u_{r m s}$ and $w_{r m s}$ are simply the statistical variances of a velocity time series.

Equations 1,2 and 3 are given in slightly different forms in the paper by Hughes (1992). The differences are a result of the choice of coordinate orientation, that is, whether the $\mathrm{x}$-axis points to the right or the left. This difference drops out in the derivation of Equations 4 and 5. Thus Equations 4 and 5 are applicable regardless of the choice of coordinate axis orientation. The difference may become important in studies of phase angle. However, in this thesis it will be shown that a random phase angle works as well as a measured phase angle in applying Equations 4 and 5 , thereby making the discrepency irrelevant.

Equations 4 and 5 were applied in this thesis by specifying the discrete wavenumbers $\left(k_{n}\right)$, discrete angular wave frequencies $\left(\sigma_{n}\right)$, and discrete amplitudes $\left(a_{n}\right)$ of the measured incident wave spectrum, along with water depth $(h)$ and spatial position $(X, Z)$. The wave number for a given water depth and wave period can be calculated exactly from the linear wave dispersion relation. However, Nielsen's (1982) approximate equation solves for $k_{n}$ explicitly and is accurate to within one percent in the range $0<h / L_{0}<0.3\left(L_{o}\right.$ is the deepwater wavelength defined as $\left.L_{o}=g T^{2} / 2 \pi\right)$. Nielsen's equation is applied in the computer program that solves Equations 4 and 5 . 


\subsection{Experimental Results}

Figures 7 to 10 compare the velocity statistics $u_{r m s}$ and $w_{r m s}$ measured by the LDV with $u_{r m s}$ and $w_{r m s}$ estimated using the measured incident wave spectrum in Equations 4 and 5. Plots were made of the ratio of measured/predicted $u_{r m s}$ and $w_{r m s}$ vs. the following parameters of the measured incident wave spectrum

- $T_{p}$

- $H_{m o}$,

- $h$, water depth,

- $z / h$,

- $H_{m o} / h$,

- $h / g T_{p}^{2}$, relative depth,

- $H_{m o} / g T_{p}^{2}$

- Ursell Number $=L_{o}^{2} H_{m o} / h^{3}$,

- $z H_{m o} / h^{2}$.

where $L_{o}$ is the deepwater wavelength associated with the peak spectral period, $T_{p}$

Of particular interest is the influence of nonlinear wave conditions. Nonlinearities may affect the ability of Equations 4 and 5 to predict $u_{r m s}$ and $w_{r m s}$. A goal of this thesis was to develop empirical corrections to Equations 4 and 5 to account for any nonlinearities. However, no clear trends are evident in the plots shown in Figures 7 to 10 (additional plots are in Appendix I, Figures 37 to 50). Two dimensionless parameters commonly correlated with nonlinearities are the Ursell number and relative depth. Figure 7 graphs measured/predicted $u_{r m s}$ vs. relative depth. Hughes (1992) produces the same plot for the case of perfect reflection from a vertical wall (rather than variable reflection from a spending beach in this study). Hughes discovered a trend whereby measured/predicted $u_{r m s}$ is underpredicted at low values of relative depth and is overpredicted at higher values. No such trend is evident in Figure 7. If anything, there may be a slight decrease for higher values of relative depth. The author has no physical explanation as to why there would be a trend in the case of a perfectly reflecting vertical wall and no trend for small reflection from a spending beach. Future investigations may 


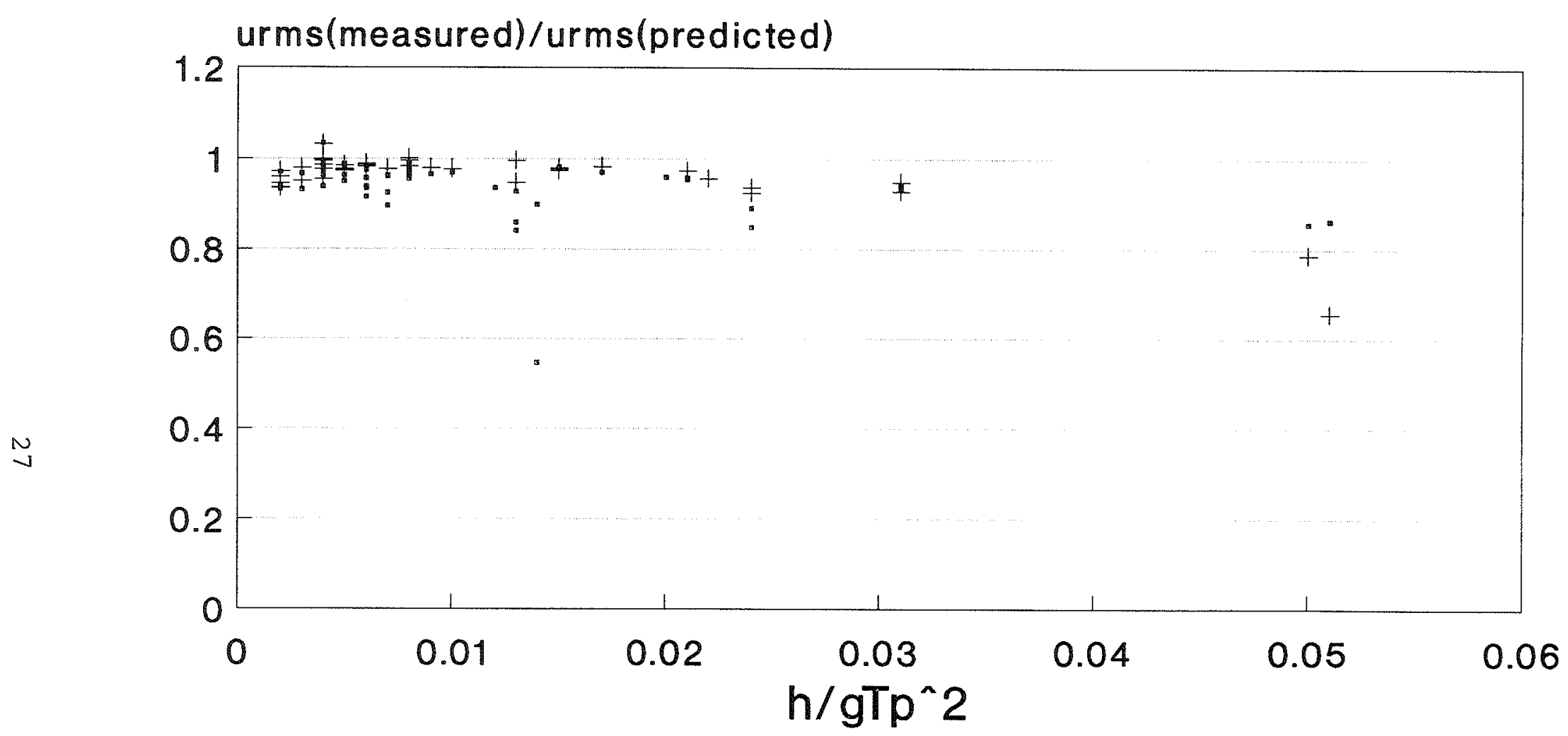

- $z$ =mid-depth $\quad+\quad z=$ near bottom

Figure 7: $u_{r m s}$ Measured/Predicted vs. Relative Depth 


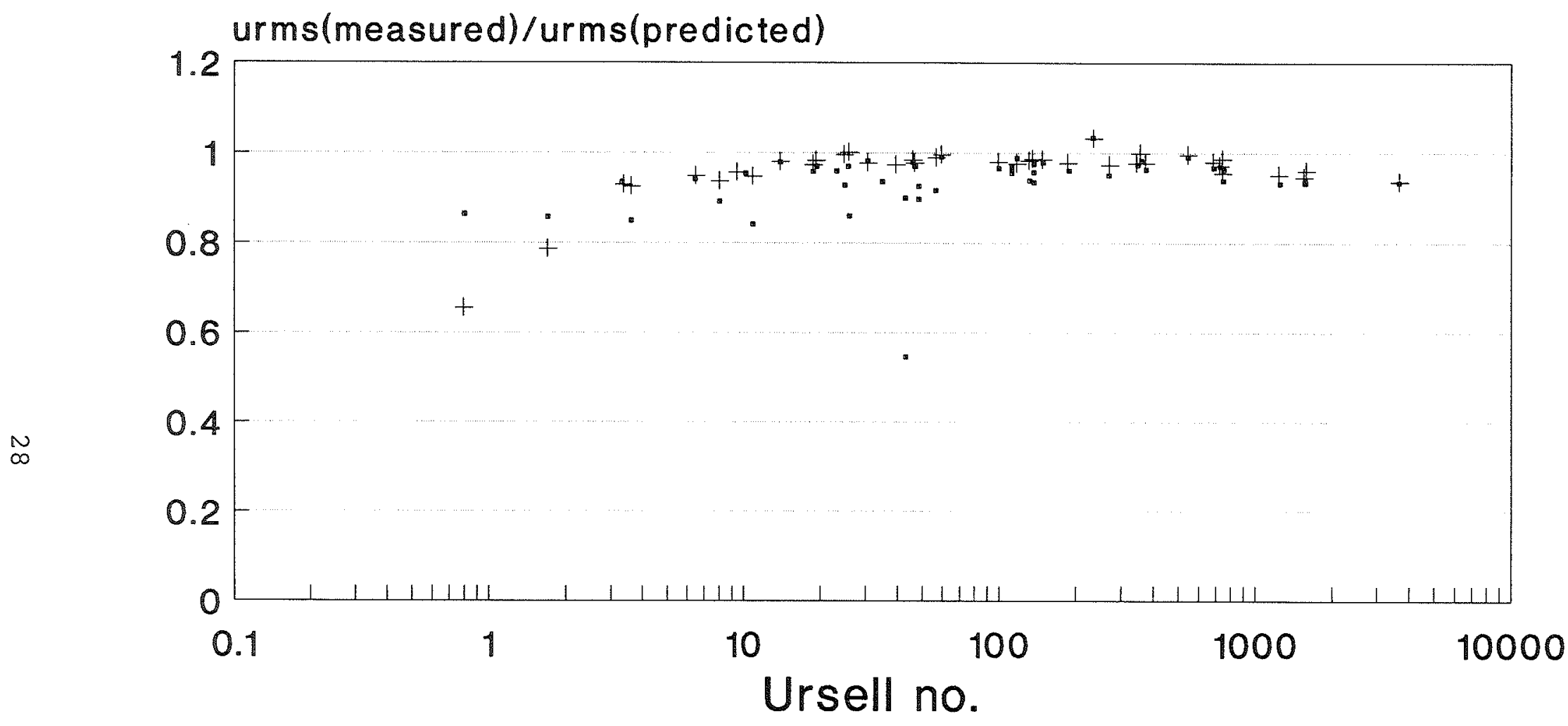

- $z$ =mid-depth $\quad+\quad z=$ near bottom

Figure 8: $u_{r m s}$ Measured/Predicted vs. Ursell No. $\left(H_{m o} L_{0}^{2} / h^{3}\right)$ 


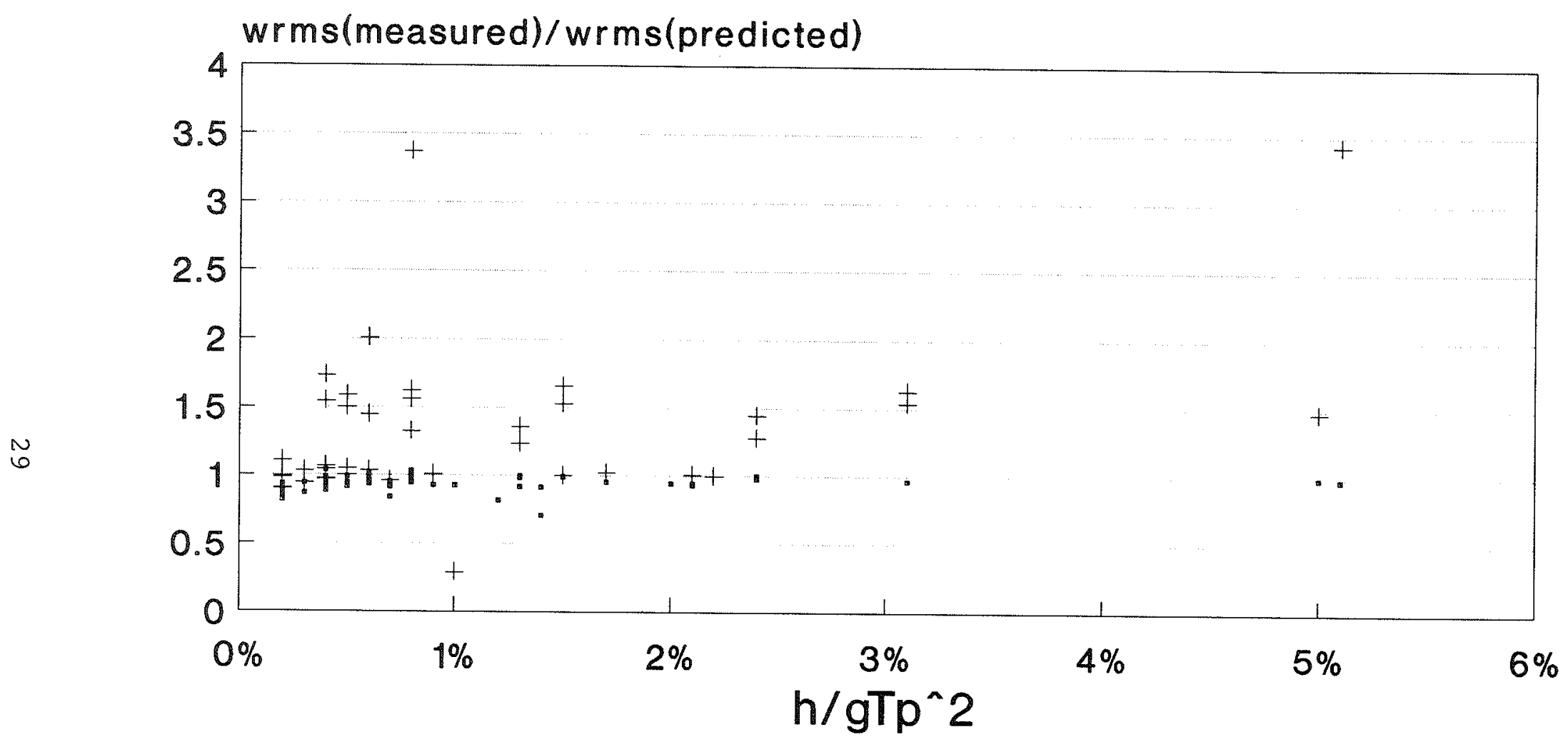

- $z=$ mid-depth $\quad+\quad z=$ near bottom

Figure 9: $w_{r m s}$ Measured/Predicted vs. Relative Depth 


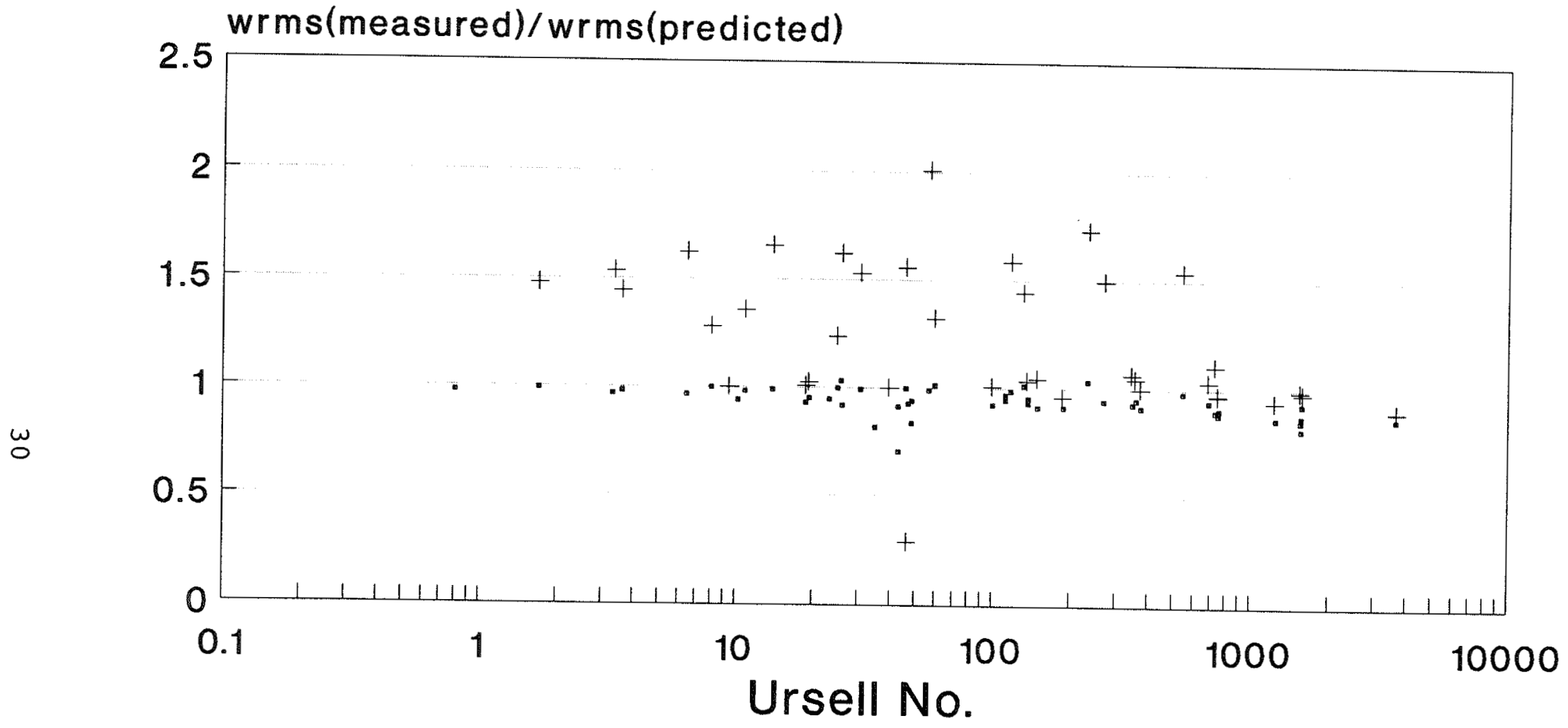

- $z=$ mid-depth $\quad+\quad z=$ near bottom

Figure 10: $w_{r m s}$ Measured/Predicted vs. Ursell No. $\left(H_{m o} L_{0}^{2} / h^{3}\right)$ 
want to look at intermediate cases of reflection coefficients between that of perfect reflection $\left(K_{r}=1.0\right)$ and small reflection $\left(K_{r} \leq 20 \%\right)$ in order to quantify any transition between the trends discovered by Hughes (1992) and the results in this thesis.

The only clear observation is that Equations 4 and 5 slightly underpredict the measured $u_{r m s}$ and $w_{r m s}$ (except for $w_{r m s}$ near bottom). The reason for predicted $u_{r m s}$ and $w_{r m s}$ being lower may be due to the effect of reflected waves "cancelling out" through linear superposition the incident wave velocities, thereby lowering the actual $u, w$ and by extension $u_{r m s}$ and $w_{r m s}$.

Figures 7 to 10 show a slight difference between points near bottom and those at mid-depth. Equations 4 and 5 underpredict the magnitude of $u_{r m s}$ and $w_{r m s}$ more at mid-depth than at the bottom. This effect agrees with the well known observation that linear wave theory becomes an increasingly poor predictor of wave properties as the wave crest is approached. The author concludes that this nonlinear effect exists throughout the water column. However, the magnitude of the difference becomes much less as the bottom is approached. One would intuitively expect some effect due to nonlinearities. The differences for water particle velocities can be expected to parallel the differences for water surface profile. However, the shape of Cnoidal and Stokes profiles is such that the variance of the surface elevations $\eta_{r m s}$ is not greatly different from linear theory, even though individual water elevation points differ. One would expect the same thing for velocity measurements and this is confirmed by this study.

Plots which apply the Ursell number (Figures 8 and 10) show similar scatter compared to plots with relative depth as the dependent variable. The lack of any strong trends and the inconsistencies described in the above paragraph leads the author to conclude that development of an empirical correction to Equations 4 and 5 based on the existing data would not be justified. It is concluded that Equations 4 and 5 provide estimates correct to within $10 \%$ based on examination of Figures 7 to 10, except for near bottom vertical velocities. For near bottom vertical velocities, $(z / h>0.85)$, the author recommends multiplying Equation 5 by 1.5 to estimate $w_{r m s}$ to within $75 \%$.

An important aspect making it difficult to quantify errors due to nonlinearities is that the incident wave spectrum is being calculated by the Goda method, which is itself based on linear wave theory. Goda and Suzuki (1976) do not rigorously quantify the errors in their method. Repeatability types of tests in Goda and Suzuki's experiments showed "dispersion" of estimated incident amplitudes about the mean on the order of $\pm 5 \%$. Plots of these tests demonstrated random errors with an approximately Gaussian distribution. 
Also of interest is that Equations 4 and 5 require specifying horizontal position, $x$, and phase angle, $\theta$. Without a reflecting structure, $K_{r_{n}}=0$ and $x$ and $\theta_{n}$ drop out. For a perfectly reflecting wall (and therefore presumably vertical), $\theta_{n}$ drops out and $x$ is the horizontal distance from the wall. However, for a sloping spending beach with variable $K_{r_{n}}$, specifying $x$ and $\theta_{n}$ is necessary. For such a case, $\theta_{n}$ is not always readily available and $x$ is not precisely defined. Figures 11 and 12 plot predicted values of $u_{r m s}$ from equation $4 \mathrm{vs}$. distance from toe of slope (values of $x$ ). Note that $u_{r m s}$ does not equal zero at $x=0$, although a cursory look at the graphs may give that impression. Two lines are plotted, one calculated using the measured phase angle and one with a random phase angle. The plots show some interesting features such as an envelope effect for the amplitudes of the varying $u_{r m s}$. Also evident is a wavelength on the order of $5.0 \mathrm{~m}$ combined with shorter wavelengths on the order of $0.2 \mathrm{~m}$. The greatest variability in $u_{r m s}$ is within one meter (one meter in the wave tank) of the toe of slope. However, the range of variability does not seem to decrease past one meter. These features are interesting but also peripheral to this thesis. The relevant points are as follows:

- The range of variability of $u_{r m s}$ and $w_{r m s}$ with varying $x$ is less than $0.05 \%$, for horizontal locations more than 1 meter from reflecting structure (in wave tank).

- There is only a minor difference between using a random phase angle, $\left(\theta_{n}\right)$, and a measured phase angle in applying Equations 4 and 5. A measured phase angle results from the Fourier analysis of the wave signal. A random phase angle is a random value between $0^{\circ}$ and $90^{\circ}$ applied in Equation 4 and 5 .

- The author recommends simply using the distance from the toe of slope for the horizontal position, $x$. For a structure with a reflection coefficient above $20 \%$, this may not be appropriate. For this case, variability of $u_{r m s}$ with $x$ may become important, as discussed by Hughes (1992). The reason for such variability can be intuitively understood by considering a regular wave reflecting from a vertical wall. The clapotis that is established has a region half a wavelength from the wall where the horizontal velocity is theoretically always zero. One quarter of a wavelength from the wall the horizontal velocity is a maximum. Thus a narrow banded spectrum will approach the behaviour of such a regular wave resulting in a non-uniform distribution of $u_{r m s}$ with $x$. A non-uniform distribution of wave height will also occur.

- The greatest variability in predicted $u_{r m s}$ is adjacent to the reflecting structure. 


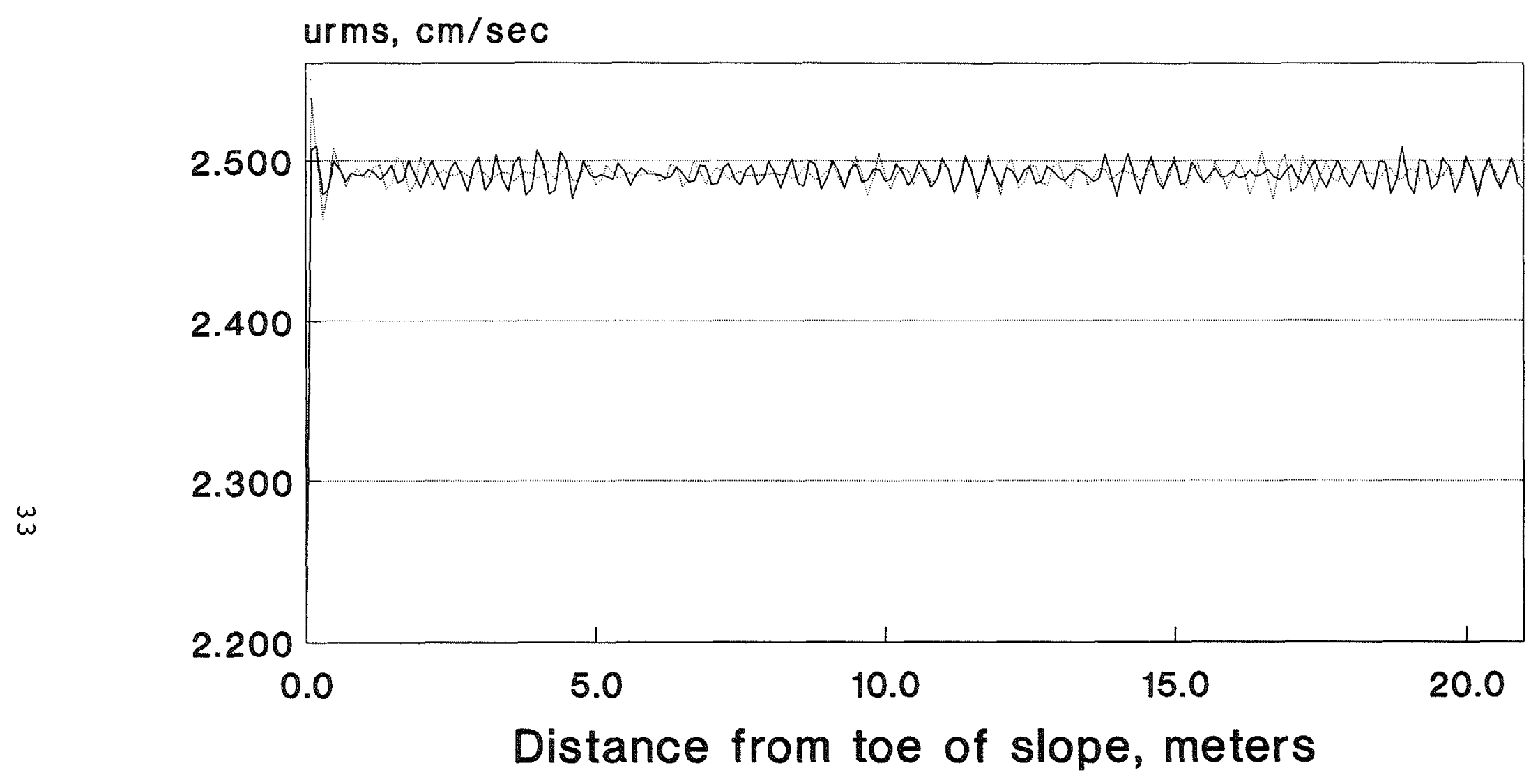

- urms measured phase urms random phase

Figure 11: Predicted $u_{r m s}$ Velocity vs. X-coordinate (Time Series No. 9). $h=15 \mathrm{~cm}, Z=25 \mathrm{~cm}, H_{m o}=4.2 \mathrm{~cm}, T_{p}=1.0 \mathrm{sec}$ 


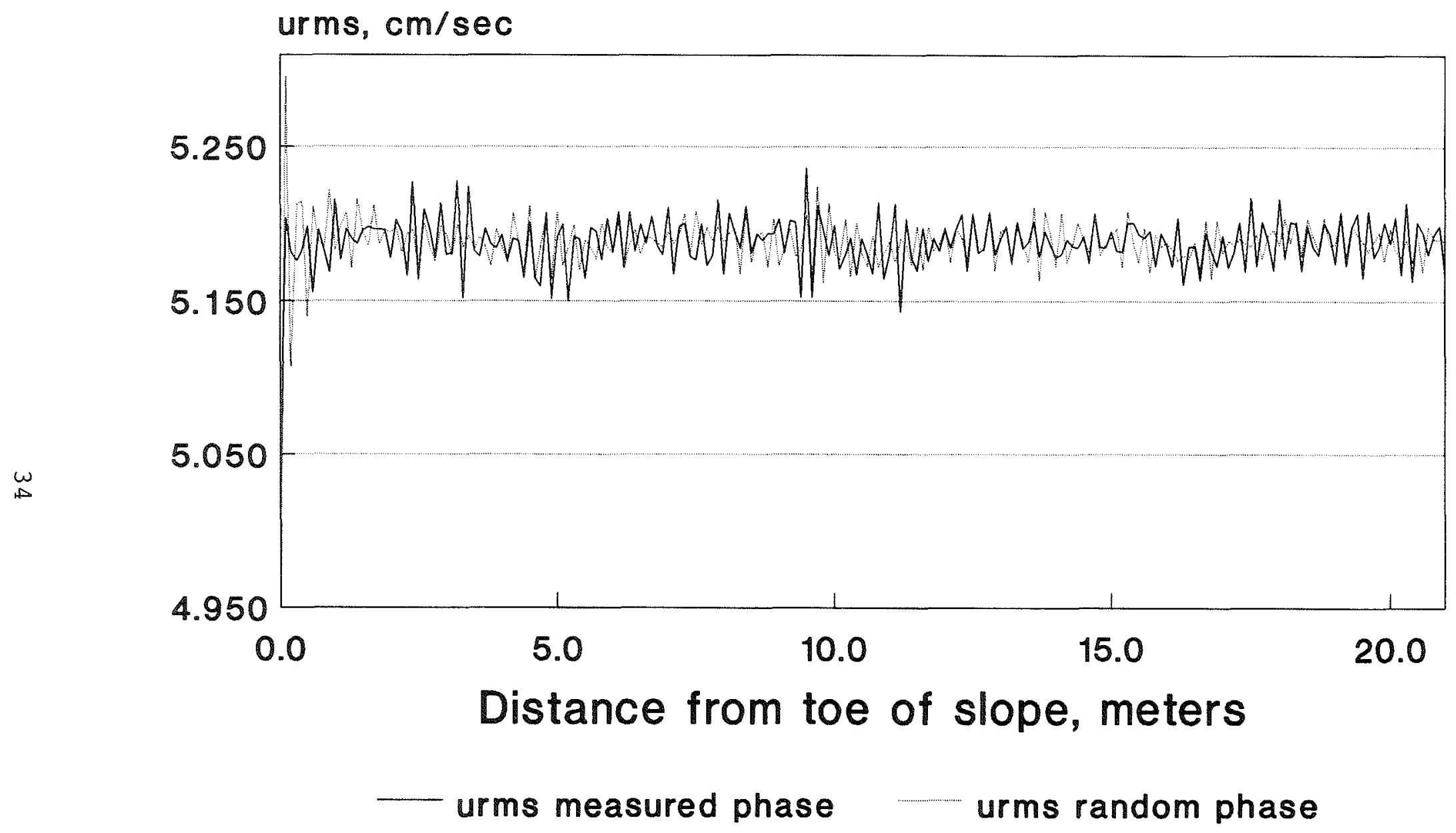

Figure 12: Predicted $u_{\tau m s}$ Velocity vs. X-coordinate (Time Series No. 69). $h=15 \mathrm{~cm}, Z=7.5 \mathrm{~cm}, H_{m o}=3.2 \mathrm{~cm}, T_{p}=1.0 \mathrm{sec}$ 
Figure 13 plots the $\%$ range of variation in predicted $u_{r m s}$ (as it varies with $x$ ) vs. relative depth for every time series. Additional plots are in Appendix I, Figures 51 to 53 . No trends are observed with respect to the relative depth $\left(h / g T_{p}^{2}\right)$. It is evident that the range of variability of $u_{r m s}$ and $w_{r m s}$ with respect to $x$ is very small for the case of $K_{r} \leq 20 \%$.

\subsection{Laboratory Effects}

Figures 14 to 17 plot the reflection coefficient, $K_{r}$, vs. various parameters. Figure 14 presents the result of regular waves that were run before the irregular wave time series. These 18 regular wave runs were made in order to verify some of the computer programs and to optimize the design of the wave absorbing (spending) beach. The final configuration of the spending-beach used three layers of "horsehair" wave absorbing mats (approximately $2.5 \mathrm{~cm}$ per mat) on top of the 1:30 concrete slope. Low $K_{r}$ values were desired for the experiments. The relatively high $K_{r}$ values reported here are in some ways disappointing. It is interesting that increased layers of horsehair mats slightly increased $K_{r}$ (Figure 14). The author suspects that vertical wave screens and/or a gravel surface on the sloping beach would have further reduced reflection.

Figure 14 depicts a trend of increasing reflection for increasing ratio of wave height to water depth. This trend is even more pronounced for the case of irregular waves (Figure 15). This trend may be partly a laboratory effect caused by the method of measuring reflection. Wave height/water depth is also directly correlated with incidents of wave breaking in the wave tank. The random superposition of wave components results in instances where individual waves are excessively high or steep resulting in wave breaking. This type of wave breaking can occur anywhere in the wave tank. The breaking of the wave results in secondary waves propagating in both directions in the wave channel. Thus, wave energy that was travelling solely in a direction incident to the beach is, through the breaking process, sent in both the incident and reflected directions thereby increasing the measured value of $K_{r}$. Higher wave heights will result in a greater number of instances of breaking waves. Since most incident waves are higher than reflected waves, the breaking process should result in a net shift of energy from incident waves to reflected waves.

Such a laboratory effect could possibly be minimized by locating the Goda array (water elevation gauges) closer to the structure (spending-beach). Note, however, that Goda and Suzuki (1976) advise locating the wave gauges used for wave resolution at a distance of more than one wavelength from both the test structure and wave board. For this reason the wave gauges used were located 


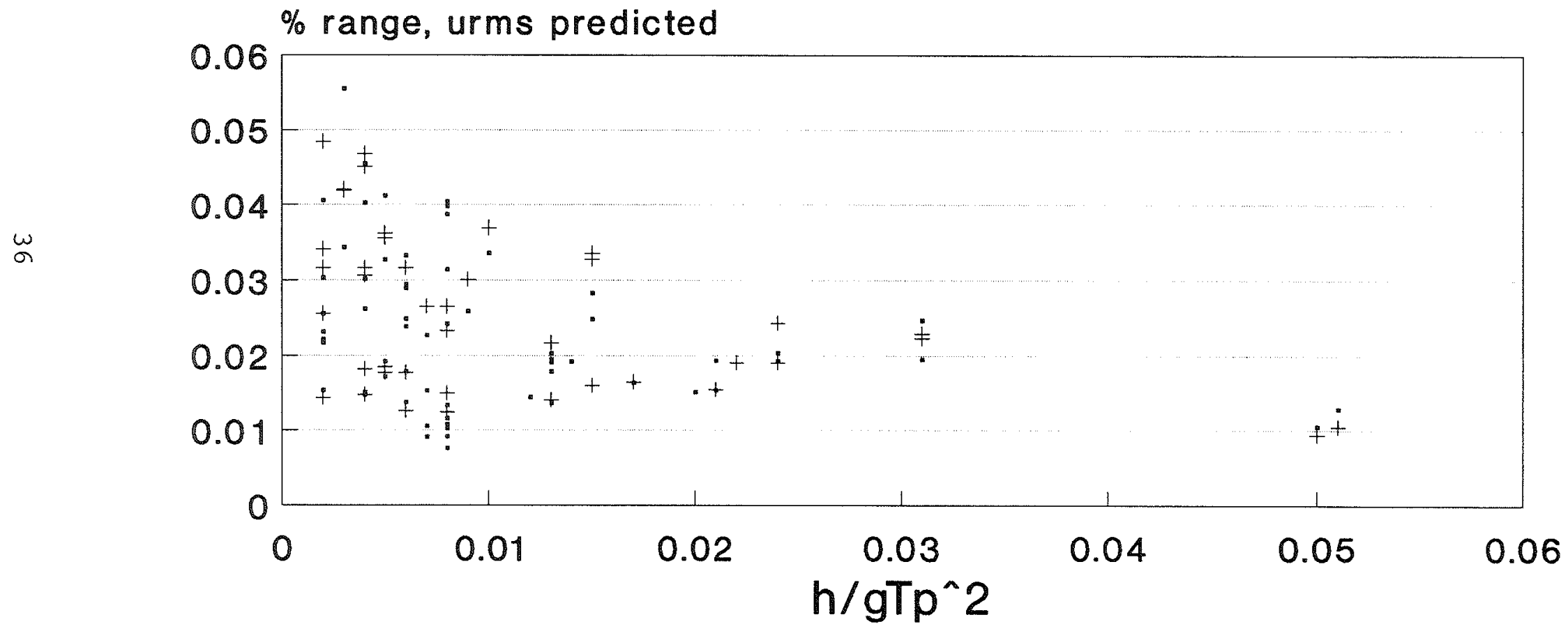

Figure 13: \%-Range of $u_{r m s}$ Predicted as a Function of X, (Random Phase Angle) vs. Relative Depth 


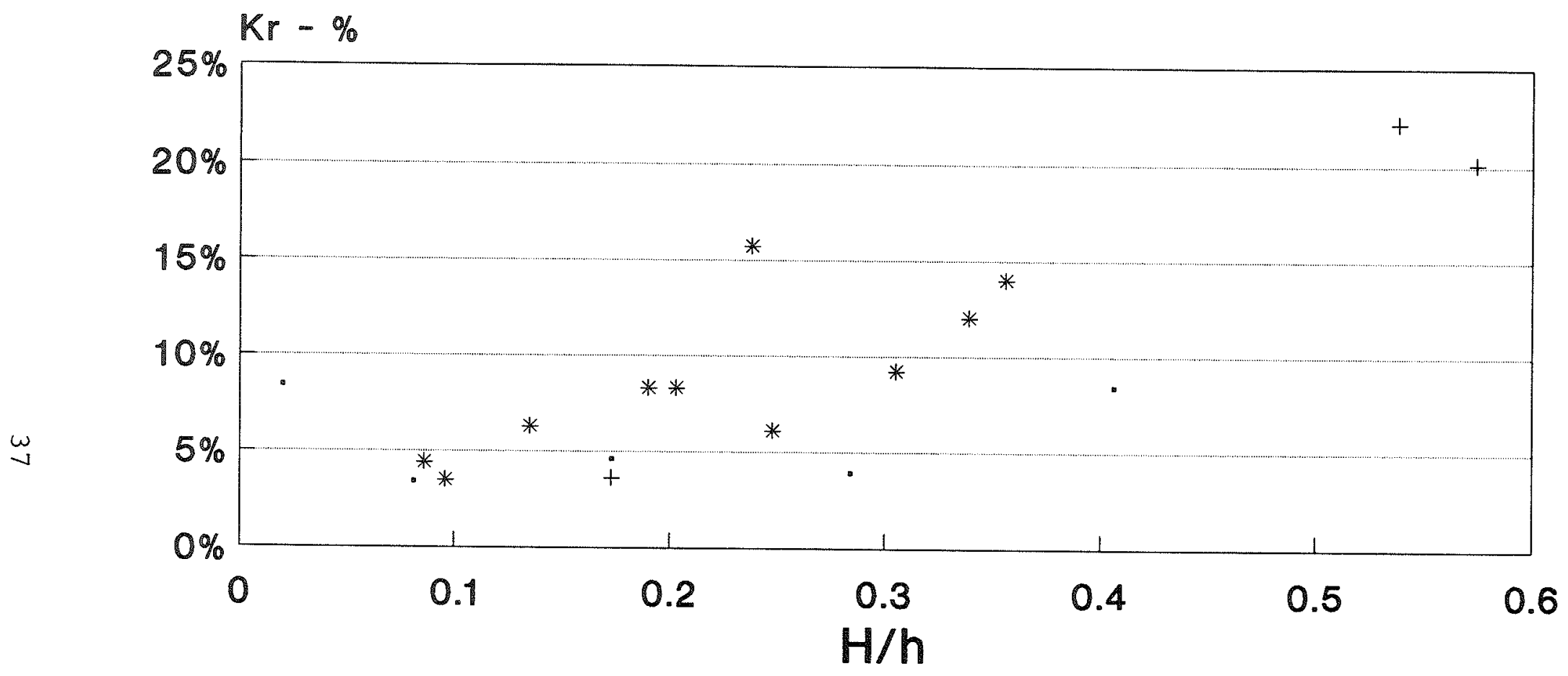

- $\mathrm{Kr}-1$ layer matting

$+\mathrm{Kr}-2$ layer matting

* $\mathrm{Kr}-3$ layer matting

Figure 14: Reflection Coefficient vs. $H / h$, Monochromatic Waves 


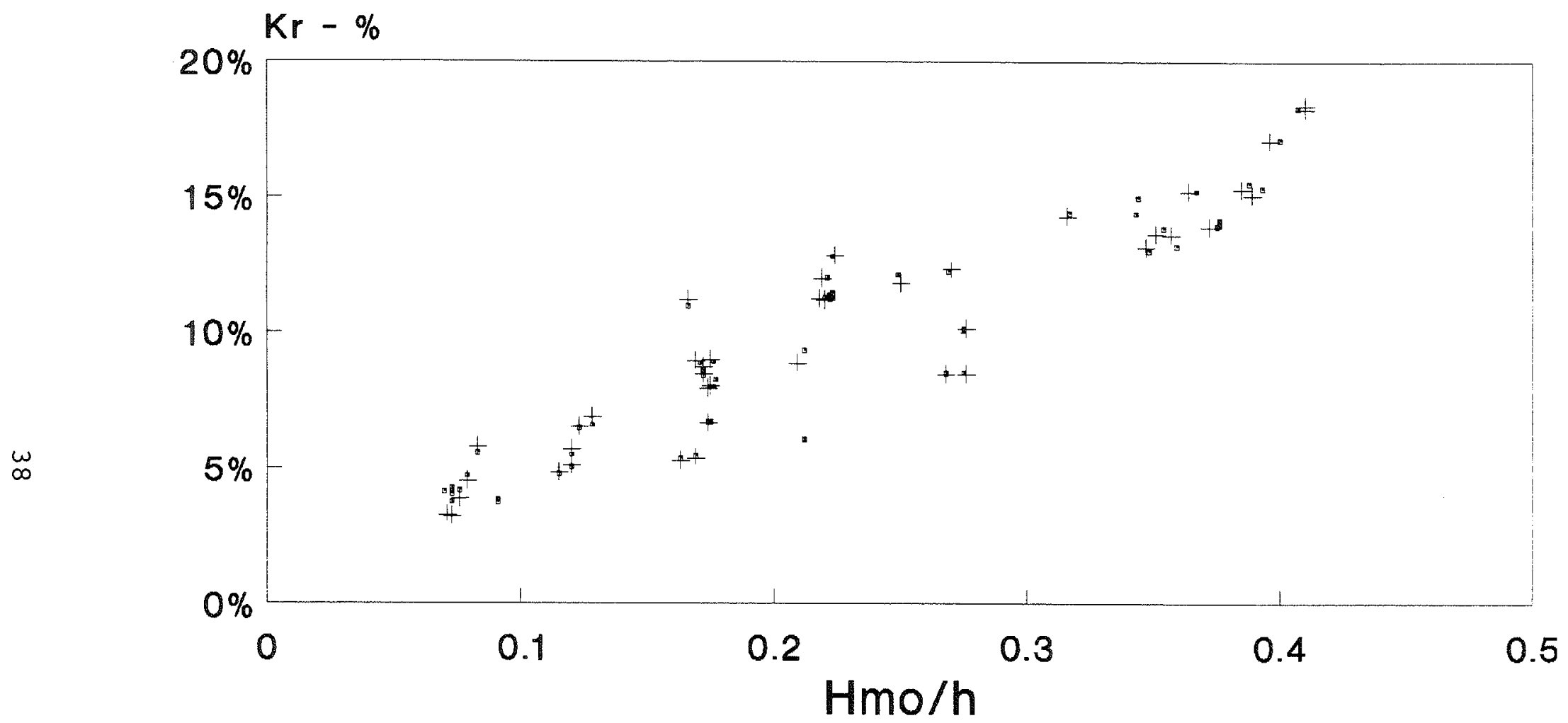

Run No. $1+$ Run No. 2

Figure 15: Reflection Coefficient vs. $H_{m o} / h$, Irregular Waves 


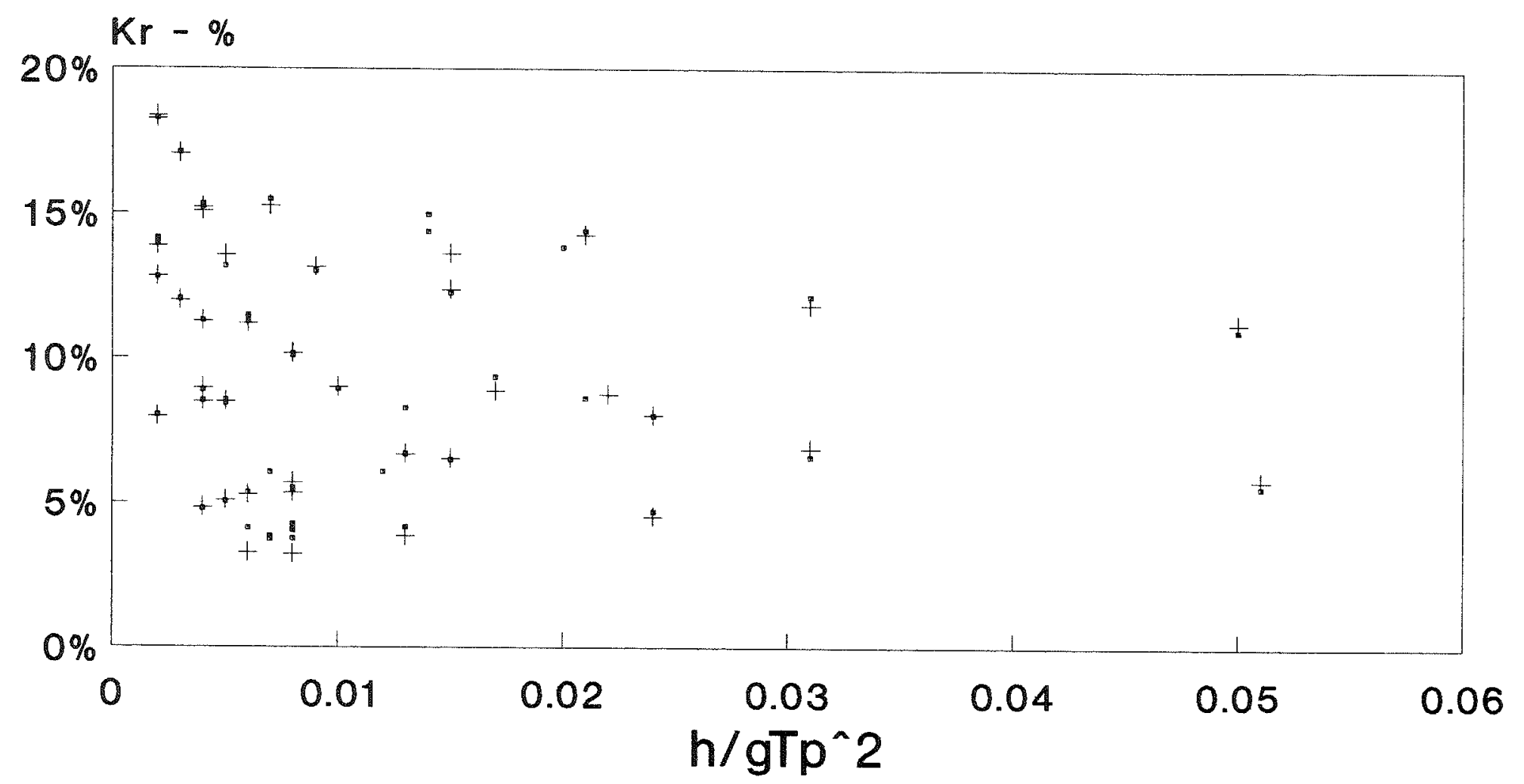

- Run No. $1+$ Run No. 2

Figure 16: Reflection Coefficient vs. Relative Depth, Irregular Waves 


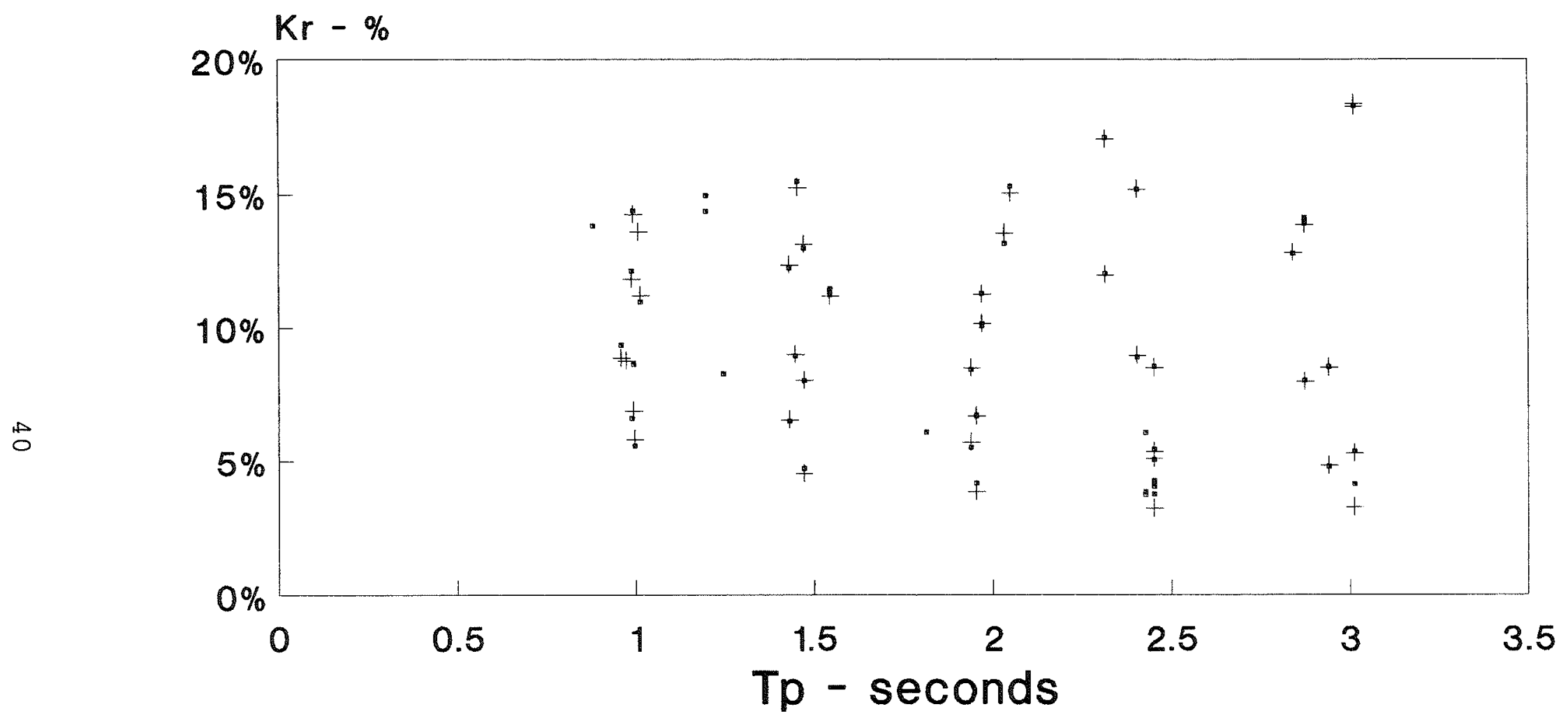

- Run No. $1+$ Run No. 2

Figure 17: Reflection Coefficient vs. Peak Spectral Period, Irregular Waves 
midway between the wave board and toe of slope.

Working against this hypothesis of distortion of $K_{r}$ by wave breaking is that the regular wave runs did not have any observed wave breaking between the Goda array and the spending beach for the case of $H / h<0.4$. Despite this, a trend is still apparent (Figure 14).

It is fortunate that $h / g T_{p}^{2}$ is not closely correlated with $K_{r}$ (Figure 16) since this dimensionless parameter is applied in empirical formulae later in this thesis. Also of interest is that $T_{p}$ is not correlated with $K_{r}$ (Figure 17). This is unusual, one would expect a strong positive correlation between $T_{p}$ and $K_{r}$ since higher frequency waves are more easily dissipated by the horsehair wave absorbing mats.

The plots show experiment repeatability by differentiating between mid-depth and near bottom LDV measuring points. (Indicated by labeling the data points either Run No. 1 or Run No. 2.) Although this distinction is not relevant to the measurement of $K_{r}$, values of $K_{r}$ are nearly identical for the theoretically identical wave conditions thereby showing good experiment repeatability.

Experiment repeatability is also indicated by time series Numbers 340,581 , 712 , and 880 . These four runs were the same as previous runs. The $\%$ differences for certain measured variables for these four repeated runs are listed in Table 3. ( $U$ and $W$ refer to peak velocities defined by a down-crossing analysis, rms and rmq refer to root-mean-square and root-mean-quad velocities, $(+)$ and $(-)$ refer to the positive and negative directions respectively. These parameters are applied later in this thesis.)

Spatial homogeneity in the X-direction describes the quality whereby experimental results are independent of horizontal distance from the spending beach. Time series numbers $341,342,582,583,713$, and 714 are spatial homogeneity tests. Wave reflection from a structure will cause some spatial variability in measured velocities and wave height statistics for the reasons explained in Section 3.1. However, no consistent modulation of the predicted value of $u_{r m s}$ is evident in Figures 11 to 12 . For low values of $K_{r}$, say less than $20 \%$, it can be concluded that the measured statistics will be nearly uniform despite varying $X$. The statistics of the spatial homogeneity tests support this conclusion. The observed variability in the measured statistics for varying horizontal location $(X)$ is comparable to that for the repeatability tests in Table 3.

A comparison of the number of zero-crossing "waves" counted separately from the particle velocity time series and the water elevation time series is depicted in Figure 18. Additional plots are in Appendix I, Figures 54 and 55. The number of 
Table 3: Experiment Repeatability

\begin{tabular}{|l|r|r|r|r|}
\hline \hline \multirow{2}{*}{$\begin{array}{l}\text { Measured } \\
\text { Variable }\end{array}$} & \multicolumn{5}{|c|}{ \% Difference - Run No. } \\
\cline { 2 - 5 }$T_{p}$ & $0.5 \%$ & 08 \& 581 & $71 \& 712$ & $88 \& 880$ \\
$H_{m o}$ & $0.2 \%$ & $0.2 \%$ & $0.5 \%$ & $0.5 \%$ \\
$K_{r}$ & $0.1 \%$ & $0.6 \%$ & $0.5 \%$ & $0.2 \%$ \\
$u_{r m s}$ & $0.1 \%$ & $0.4 \%$ & $0.7 \%$ & $0.7 \%$ \\
$w_{r m s}$ & $0.3 \%$ & $1.6 \%$ & $1.9 \%$ & $0.2 \%$ \\
$U_{r m s}(+)$ & $0.2 \%$ & $3.0 \%$ & $0.2 \%$ & $0.4 \%$ \\
$U_{r m s}(-)$ & $0.3 \%$ & $0.3 \%$ & $0.2 \%$ & $0.7 \%$ \\
$W_{r m s}(+)$ & $1.5 \%$ & $8.0 \%$ & $23 \%$ & $0.2 \%$ \\
$W_{r m s}(-)$ & $1.0 \%$ & $10 \%$ & $1.6 \%$ & $5.0 \%$ \\
$U_{r m q}(+)$ & $0.3 \%$ & $9.5 \%$ & $0.1 \%$ & $2.9 \%$ \\
$U_{r m q}(-)$ & $0.4 \%$ & $1.4 \%$ & $6.4 \%$ & $0.6 \%$ \\
$W_{r m q}(+)$ & $3.0 \%$ & $7.5 \%$ & $3.1 \%$ & $0.2 \%$ \\
\hline$h$ & $30 \mathrm{~cm}$ & $20 \mathrm{~cm}$ & $15 \mathrm{~cm}$ & $15 \mathrm{~cm}$ \\
$z$ & mid-depth & mid-depth & mid-depth & near bottom \\
$H_{m o}$ & $8.25 \mathrm{~cm}$ & $7.51 \mathrm{~cm}$ & $3.33 \mathrm{~cm}$ & $6.15 \mathrm{~cm}$ \\
$T_{p}$ & $1.97 \mathrm{sec}$ & $2.87 \mathrm{sec}$ & $1.54 \mathrm{sec}$ & $3.01 \mathrm{scc}$ \\
\hline \hline
\end{tabular}


waves has been prorated to account for the slightly different record lengths. Each wave is by two consecutive downcrossings of the mean. The number of velocity waves measured by the LDV and the number of water elevation waves measured by the wave gauges should theoretically be the same. For horizontal velocities, the number of waves counted was approximately $20 \%$ less than the number of surface waves. For vertical velocities, some interesting trends are evident with respect to increasing nonlinearities (discussed further below).

The velocity waves were counted after the time series had undergone a bandpass filter of $0.20-5.00$ seconds (Figure 6). This filtering removed high frequency oscillations in the velocity time series caused by turbulence and imperfections in the electronic data acquisition systems. Low frequency oscillations were also removed. The effect of filtering is to decrease the number of "velocity" waves counted. Note that for turbulence studies it may be desirable to retain the effects of turbulent fluctuations.

Figure 18 provides a useful check on the quality of the measurements and the effectiveness of filtering. Large discrepancies between wave counts for velocities and water elevations is a warning that something may be out of order. The author concludes that all experiment measurements were high quality based on these wave counts as well as a visual examination of parts of every velocity time series (Figure 6). A discrepancy of more than $50 \%$ could be considered "poor" quality for this type of study. The author has no analytical explanation for the trend observed in Figure 18b (increasing number of vertical velocity waves with decreasing relative depth). Relative depth is commonly used to indicate nonlinearities. The plot shows an increase in the number of "velocity" waves with increasing nonlinearities. One can conclude from this that up/down-crossing analyses have limited usefulness in studies requiring precise statistics or attempting to show subtle effects. 
No. Waves(heights)/No. Waves(velocities)

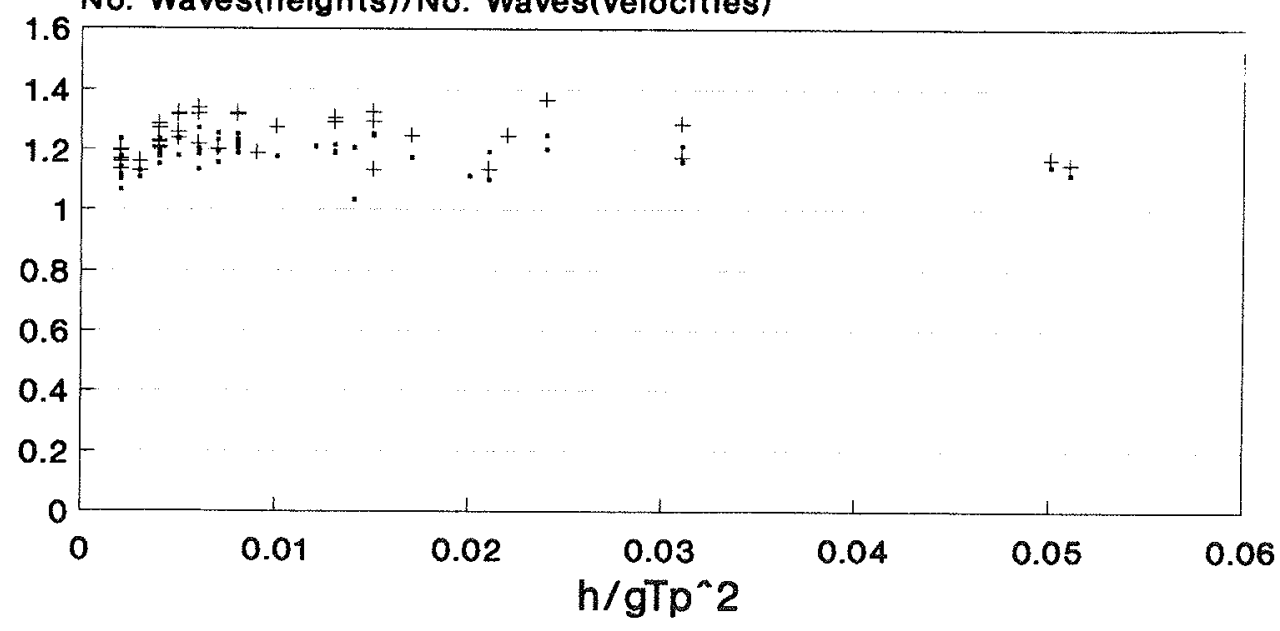

- $U, z$ amid-depth $+U, z$ mear bottom

(a) Horizontal Velocities

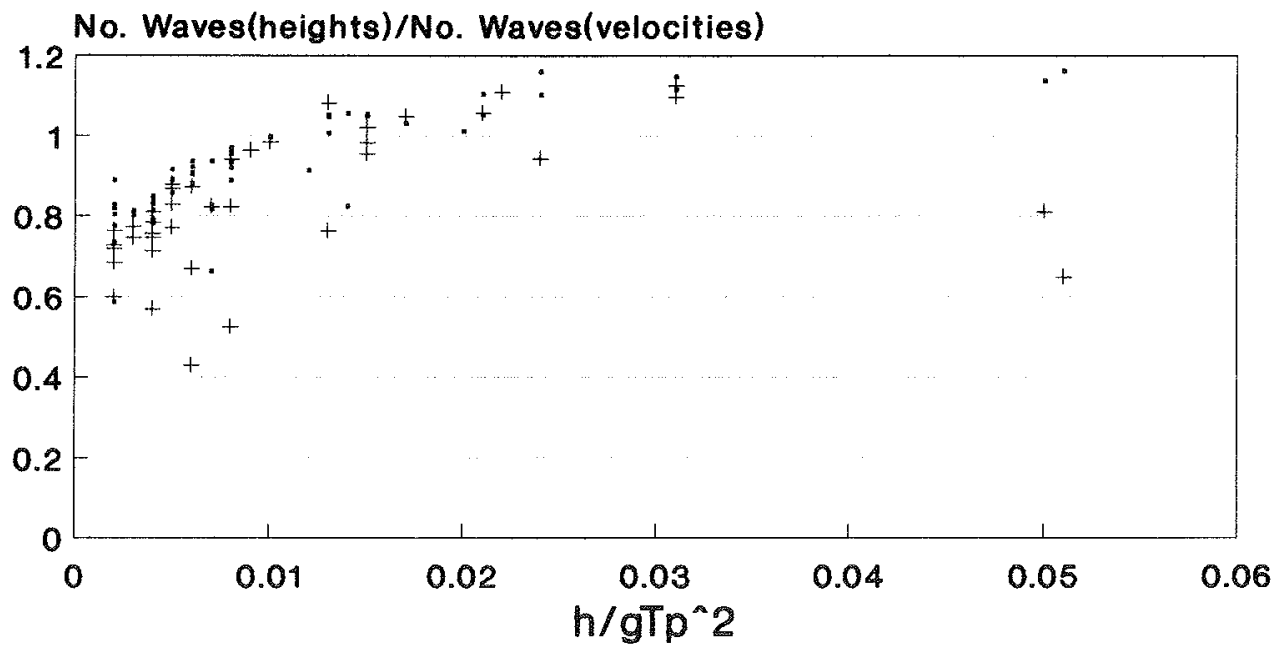

- $W_{,}$z"mid-depth $+W_{,}$zanear bottom

(b) Vertical Velocities

Figure 18: Number of Waves Counted for Water Elevation Time Series and Water Particle Velocity Time Series: (a) Horizontal Velocities; (b) Vertical Velocities 


\section{VELOCITY DISTRIBUTION}

Linear irregular wave theory assumes that the time series of sea surface elevations is the result of linear superposition of many sinusoidal wave components, each expressed by Equation 1. That is, each component has a unique frequency, amplitude, wave length, phase angle and reflection coefficient and these components do not interact. Also assumed is that sea surface elevation, $\eta(t)$, at a fixed point in space is a random process with a Gaussian (Normal) distribution.

The time series of fluid particle velocity also can be assumed to be the result of linear superposition of many sinusoidal components. Each component being expressed by Equation 2 or 3 . Also, it can be reasonably assumed that $u(t)$ and $w(t)$ follow a Gaussian distribution with zero mean and standard deviation $\left(u_{r m s}\right.$ and $\left.w_{r m s}\right)$ given by Equations 4 and 5 (Hughes 1992). The development of a probability distribution for particle velocity will parallel that for sea surface elevation.

\subsection{Gram-Charlier Distribution}

Longuet-Higgins (1963) developed a probability distribution function applicable to non-Gaussian, nonlinear ocean waves through a modification of the normal distribution (O.S. Rice (1944) originally developed much of the theories relating to the mathematical description of wave phenomenon). The equation (in standardized form, after dividing by the standard deviation of $\mathrm{x}$ ) is given by Ochi (1982) as follows:

$$
f(x)=\frac{\exp \left(\frac{-x^{2}}{2}\right)}{\sqrt{2 \pi}}\left[1+\frac{\lambda_{3}}{3 !} H_{3}(x)+\frac{\lambda_{4}}{4 !} H_{4}(x)+\frac{\lambda_{3}^{2}}{72} H_{6}(x)+\frac{\lambda_{5}}{5 !} H_{5}(x)+\cdots\right]
$$

where

$x=$ nondimensional parameter given by $(x-\bar{x}) / \sigma$ where " $\mathrm{x}$ " is the variable of interest $(\eta, u$, etc. $)$, and $\sigma$ is the standard deviation of the variable.

$H_{n} \quad=$ Hermite polynomial of degree $n$,

$\lambda_{3}=$ skewness (non-dimensional),

$\lambda_{4}=$ kurtosis (non-dimensional),

$\lambda_{5} \quad=$ higher order moment of the nondimensional distribution.

This equation will be referred to henceforth as the Gram-Charlier distribution. 
Different probability. density functions are defined by including different terms of the series in Equation 6 (Ochi 1982). The following four distributions were studied:

Gaussian distribution: First term of the series in Equation 6 Nonlinear (I): Up to the third term of the series in Equation 6 Nonlinear (II): Up to the fourth term of the series in Equation 6 Nonlinear (III): All five terms of the series in Equation 6

Note that the Hermite polynomials used in Equation 6 are not the ones typically found in math references (CRC Standard Mathematical Tables 1987). LonguetHiggins (1963) applies the following version

$$
\begin{aligned}
& H_{n}=x^{n}-\frac{n(n-1)}{1 !} \frac{x^{n-2}}{2}+\frac{n(n-1)(n-2)(n-3)}{2 !} \frac{x^{n-4}}{2^{2}}-\cdots, \\
& H_{3}=x^{3}-3 x \\
& H_{4}=x^{4}-6 x^{2}+3 \\
& H_{5}=x^{5}-10 x^{3}+15 x \\
& H_{6}=x^{6}-15 x^{4}+45 x^{2}-15 .
\end{aligned}
$$

Application of the Hermite polynomials given in the CRC Tables does not work.

The results of previous investigations using this distribution are discussed in Section 1.1 of this thesis. Ochi (1982) outlines the derivation of Equation 6, which involves application of the cumulants-generating function.

Note that Equation 6 is close to a similar equation using Gram-Charlier series (Cramér 1946) defined as

$$
\begin{aligned}
& f(x)= \\
& \quad \frac{\exp \left(\frac{-x^{2}}{2}\right)}{\sqrt{2 \pi}}\left[1+\frac{\lambda_{3}}{3 !} H_{3}(x)+\frac{\left(\lambda_{4}-3\right)}{4 !} H_{4}(x)+\frac{\left(\lambda_{5}-10 \lambda_{3}\right)}{5 !} H_{5}(x)+\cdots\right](12)
\end{aligned}
$$

Application of Equation 6 to the measured velocity data results in a worse fit to the data than application of the plain Gaussian distribution. Figures 19 and 20 show typical results for this study. Additional plots are in Appendix I, Figures 73 to 96 . The theoretical probability distribution underestimates the number of 
higher magnitude velocity points and overestimates the number of points near zero. Figure 4 is a sample hardcopy output from the LDV microcomputer. It includes the parameters used to calculate the theoretical distributions as well as tabulations of the measured and theoretical distributions. The tabulated data is plotted in graphs like Figures 19 and 20. Under no conditions does the GramCharlier distribution provide a better fit than the Gaussian distribution.

Figure 21 plots \%-RMS vs. relative depth for each time series. The value of \%RMS error is calculated for each time series as follows; The difference between the theoretical and measured probability density for every non-dimensional velocity point is calculated. The differences are squared and then the sum of these squares is divided by the number of points. The square root of this number is the RMS error. Multiplying the RMS error by the number of points gives the \%-RMS error. Using the \%-RMS error instead of the plain RMS error is not really necessary for comparing the Gram-Charlier distribution applied in this thesis. However, the $\%$-RMS error needs to be used for the case of an inconsistent number of points, such as for the Beta-Rayleigh distribution later in this thesis.

The velocity time series applied in this thesis were unfiltered (raw data). Figures 22 and 23 compare theoretical and measured probability distributions for filtered and unfiltered data. The differences are not significant, especially when comparing the relative differences between the Gaussian (linear) and nonlinear distributions. Additional plots are contained in Appendix II.

One question is whether the author has implemented the Gram-Charlier distribution correctly. Unfortunately, the expression does not transform itself into the plain Gaussian distribution for certain theoretical values of the parameters $\lambda_{3}, \lambda_{4}$, and $\lambda_{5}$. This makes verification difficult. The fact that the probability distribution sums to 1.0 (Figure 4) is one check on the implementation although not foolproof. Another check is the results of previous investigations (Section 1.1). Ochi (1982) reproduces the results of one time series made by Honda and Mitsuyasu (1976) for wave accelerations. The relative shape of the probability distribution curves is very similar to the shapes in this thesis.

Since the Gram-Charlier distribution fits poorly the measured velocity distribution, no attempt has been made to relate the parameters of the distribution to parameters of the wave field. 

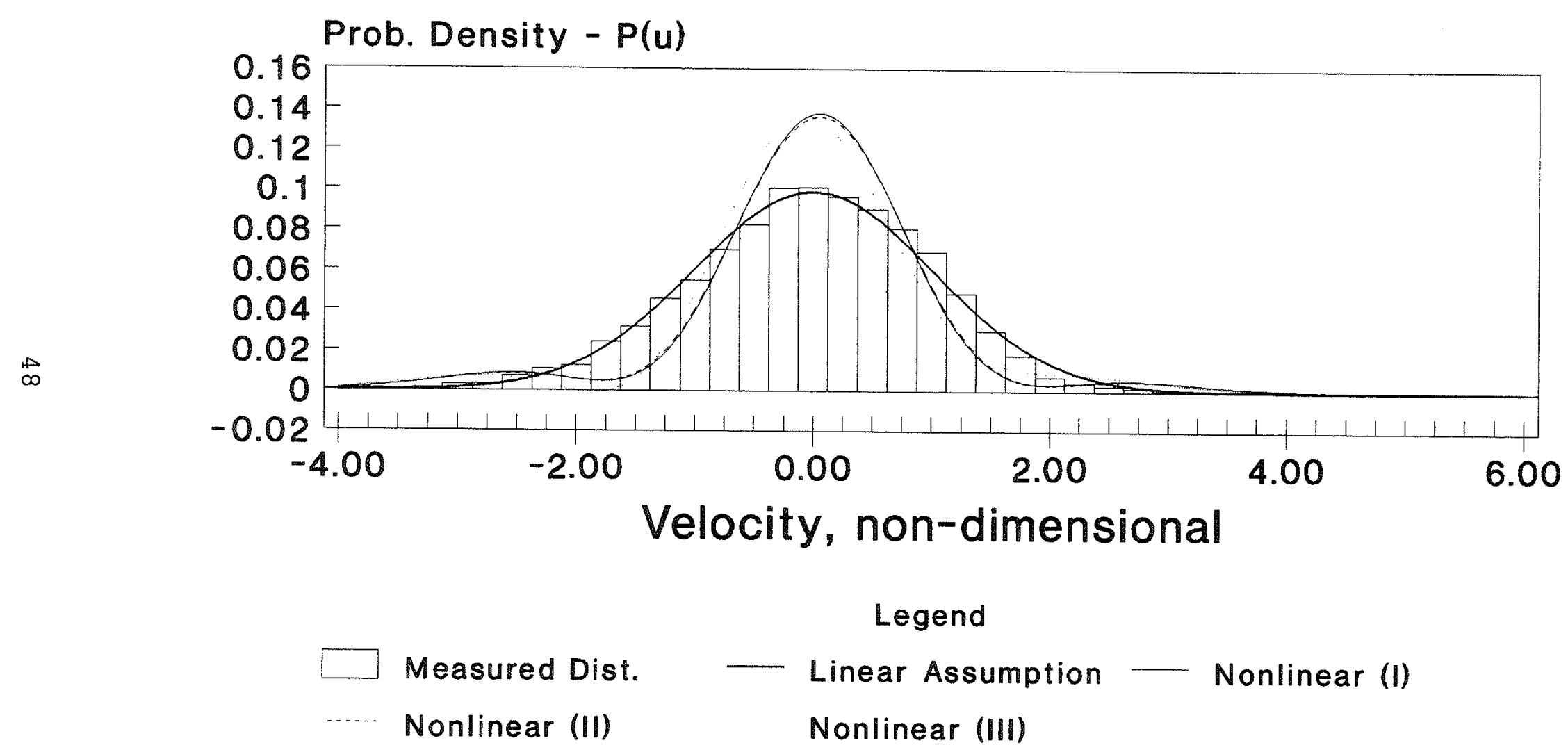

Figure 19: Horizontal Velocity Probability Distribution 


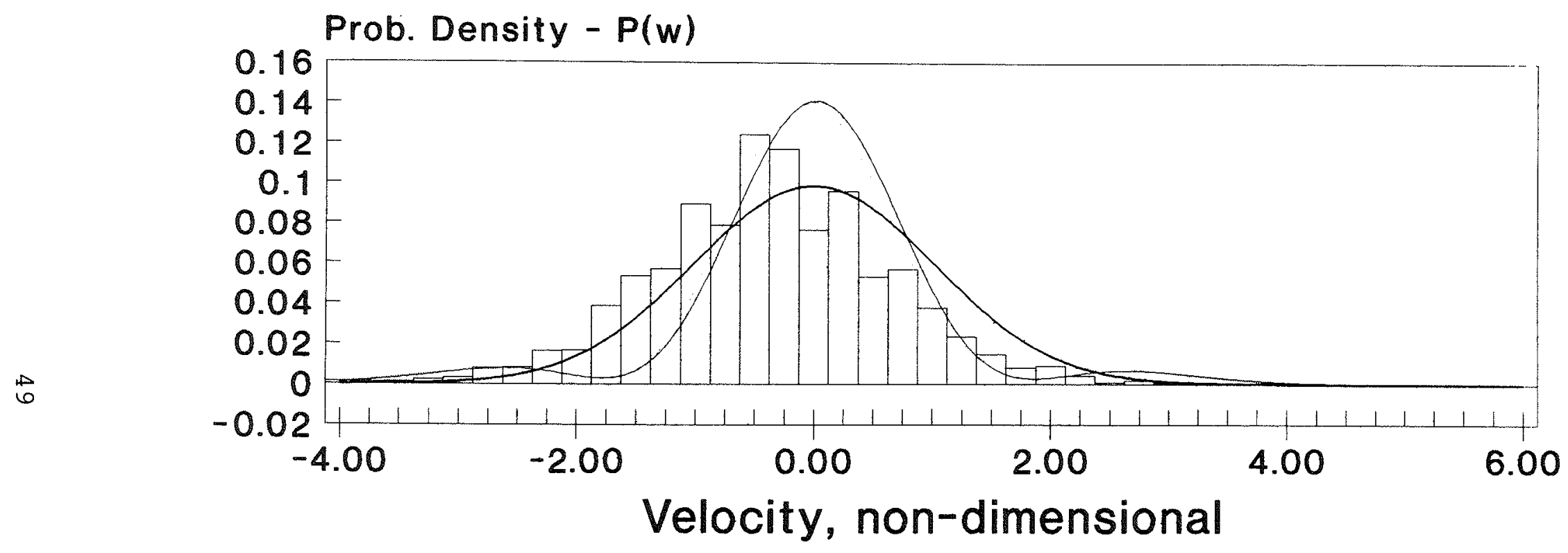

Figure 20: Vertical Velocity Probability Distribution 


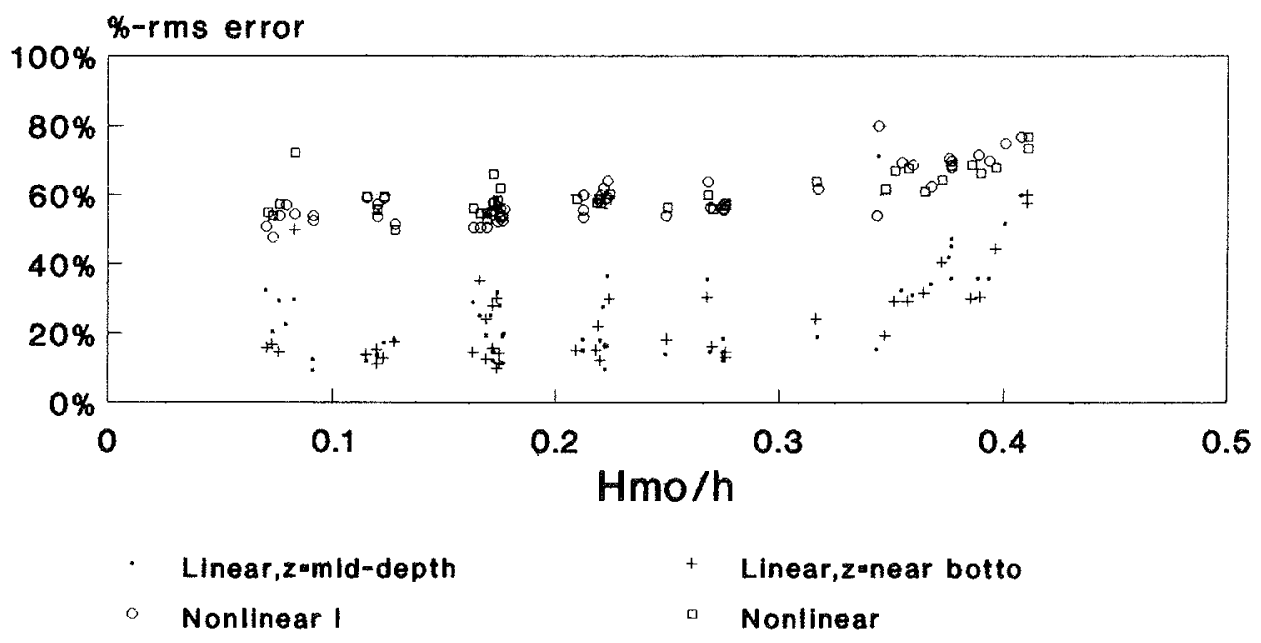

(a) Horizontal Direction

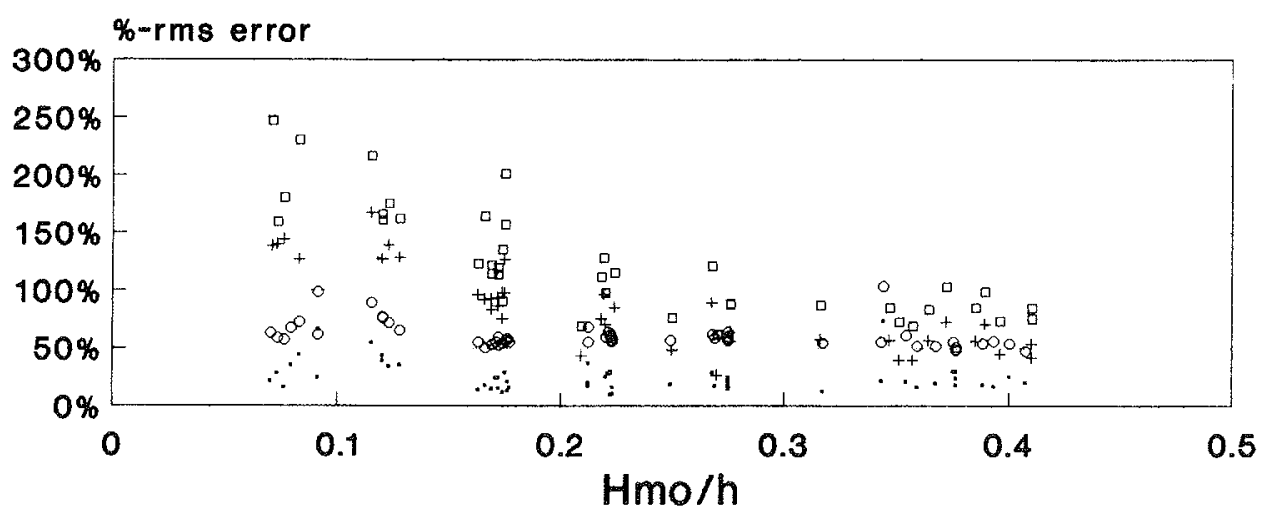

\section{Linear, z-mld-depth}

Nonlinear 1
+ Linear,zønear bottom

- Nonlinear 1

(b) Vertical Direction

Figure 21: \%-Root-mean-square Error, Gram-Charlier Distribution vs. $H_{m o} / h$. (a) Horizontal Direction; (b) Vertical Direction 


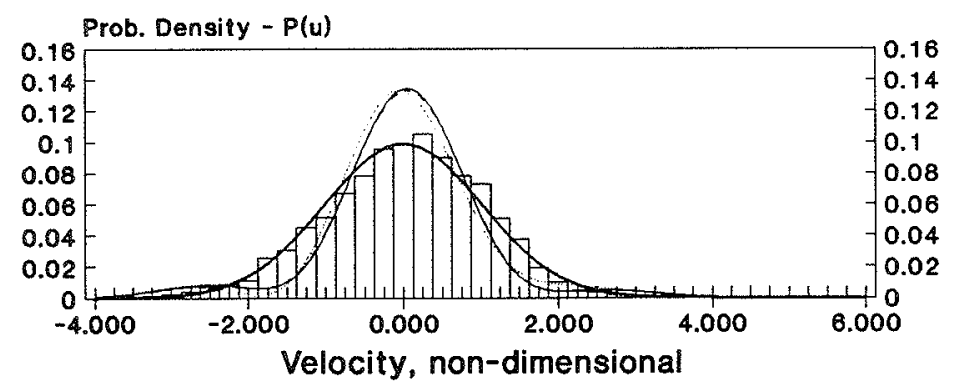

(a) Raw Data

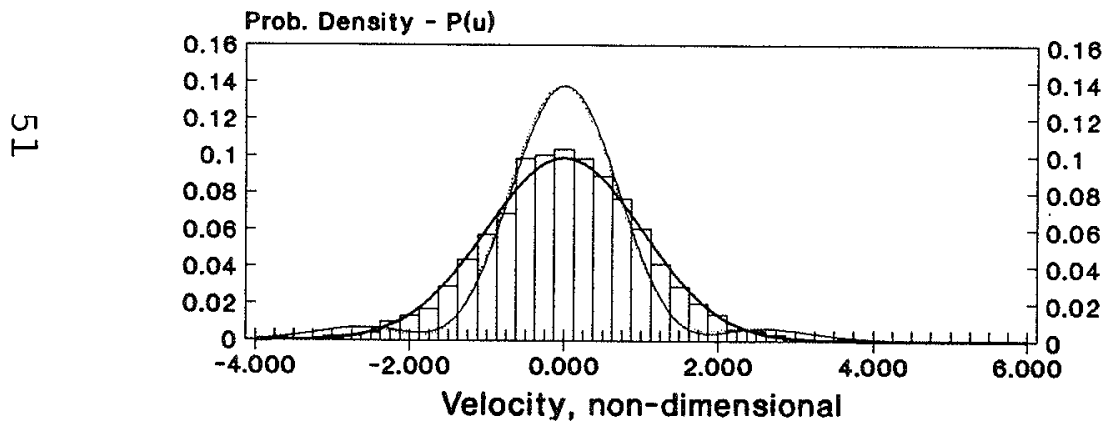

(c) Filtered Data $[0.20-10.00] \mathrm{s}$

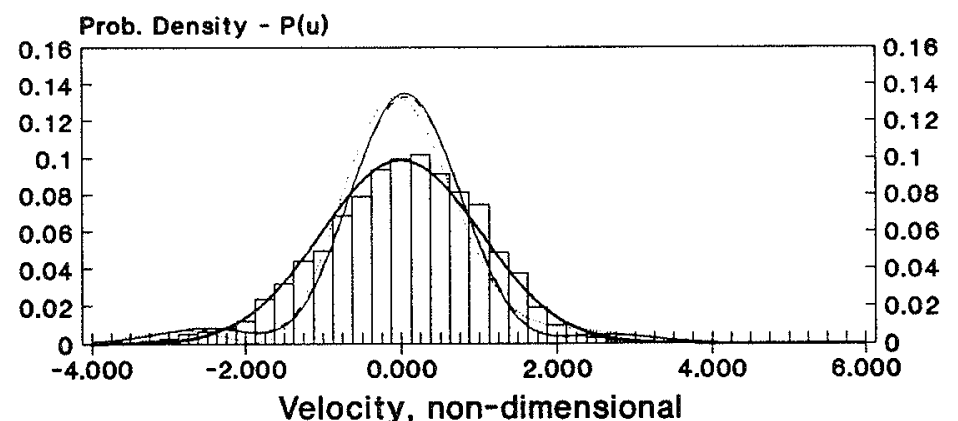

(b) Filtered Data $[0.20-5.00] \mathrm{s}$

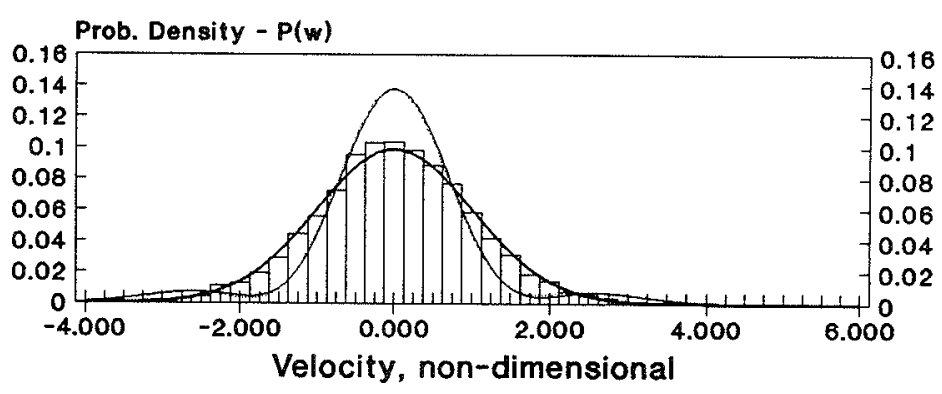

(d) Filtered Data $[0.10-5.00] \mathrm{s}$

Legend

$$
\begin{aligned}
& \square \text { Moseured Dist. } \quad \text { Linear Assumption - Nonlinear (I) } \\
& \text { Nonlinesr (III) }
\end{aligned}
$$

Figure 22: The Effect of Varying Filter Bandwidths on Fitting the Gram-Charlier Distribution to Horizontal Velocities, Time Series No. 37. (a) Raw Data; (b) Filtered Data [0.20-5.00] s; (c) Filtered Data [0.20-10.00] s; (d) Filtered Data $[0.10-5.00] \mathrm{s}$ 

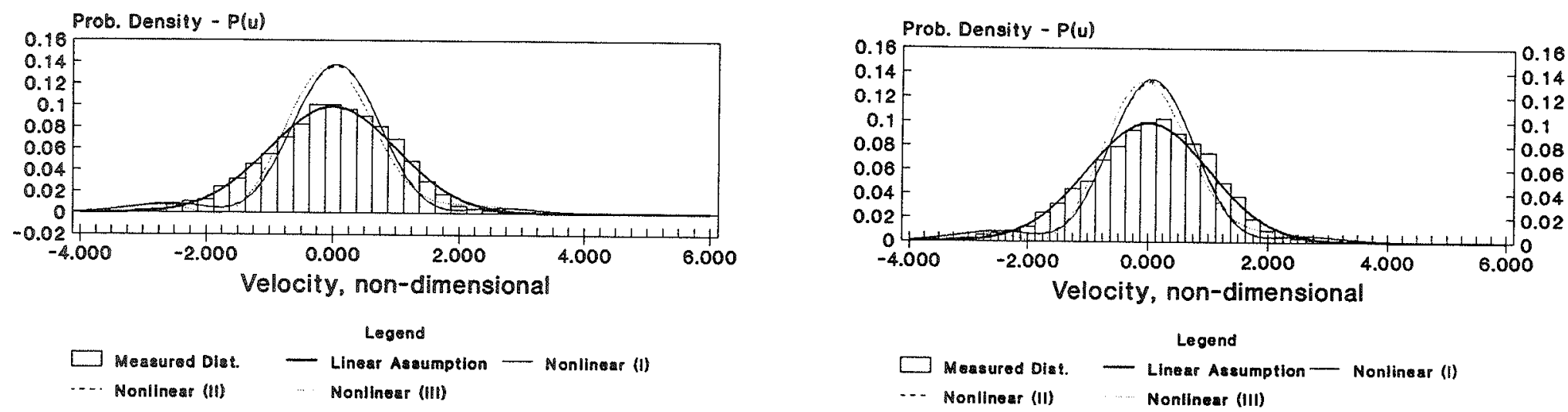

(a) Raw Data

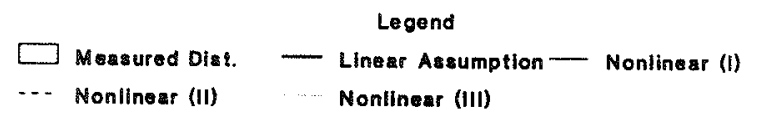

(b) Filtered Data $[0.20-5.00] \mathrm{s}$
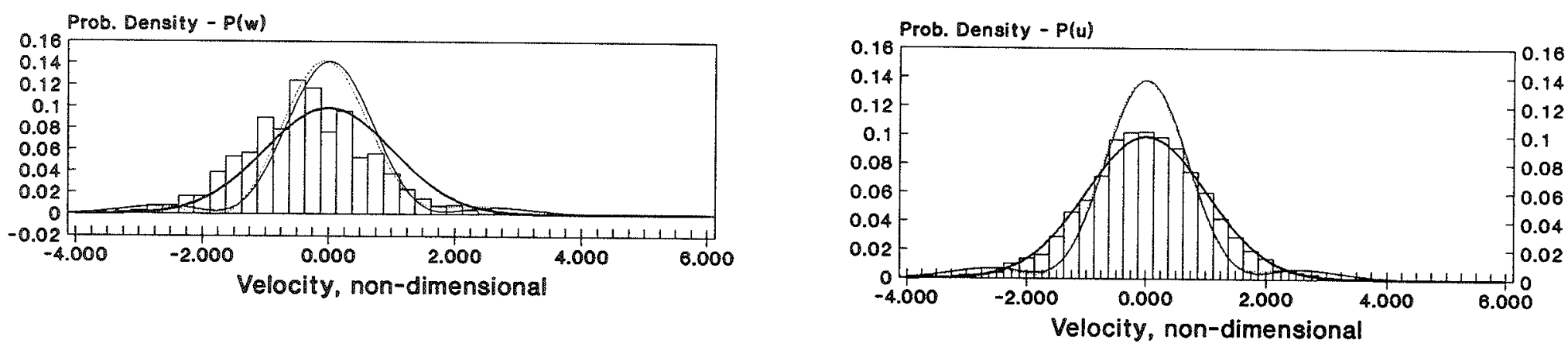

(c) Filtered Data $[0.20-10.00] \mathrm{s}$

(d) Filtered Data $[0.10-5.00] \mathrm{s}$

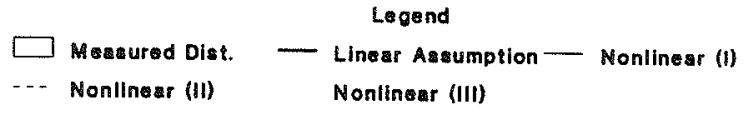

Figure 23: The Effect of Varying Filter Bandwidths on Fitting the Gram-Charlier Distribution to Vertical Velocities, Time Series No. 37. (a) Raw Data; (b) Filtered Data [0.20-5.00] s; (c) Filtered Data [0.20-10.00] s; (d) Filtered Data [0.10-5.00] s 


\section{PEAK VELOCITY DISTRIBUTION}

Predicting the magnitude of wave height as a function of statistical parameters has been a fundamental goal of much research involving random seas (wave height is defined as the vertical distance from a wave crest to a successive trough). It can be derived theoretically that the statistical distribution of wave heights (surface waves) follows the Rayleigh probability law if one assumes the following (LonguetHiggins 1952):

- The time series of water surface elevations is the result of the linear superposition of many sinusoidal components.

- At a fixed location, the water surface elevation follows a Gaussian distribution.

- The spectrum of water elevations is narrow banded. That is, the frequencies of the sinusoidal components are close together.

- The wave peaks are statistically independent.

The Rayleigh distribution has a good history of predicting wave heights even for sea conditions well outside the theoretical limits listed above. This one-parameter distribution is defined as

$$
P(H)=\frac{2 H}{H_{r m s}^{2}} \exp \left[-\left(\frac{H}{H_{r m s}}\right)^{2}\right]
$$

where

$$
\begin{aligned}
& P(H)=\text { Probability density function for wave heights, } \\
& H \quad=\text { Wave height } \\
& H_{r m s}=\text { Root-mean-squared wave height. }
\end{aligned}
$$

\subsection{The Beta Rayleigh Distribution}

Hughes and Borgman (1987) applied the "Beta-Rayleigh" distribution that modifies the above equation as follows

$$
P_{B R}(H)=\frac{2 \Gamma(\alpha+\beta)}{\Gamma(\alpha) \Gamma(\beta)} \frac{H^{2 \alpha-1}}{H_{b}^{2 \alpha}}\left(1-\frac{H^{2}}{H_{b}^{2}}\right)^{\beta-1}, \quad 0<H<H_{b}
$$


where

$H_{b} \quad=$ Breaking wave height (the maximum wave height possible), $\Gamma(\cdot)=$ The Gamma function.

and

$$
\begin{aligned}
\alpha & =\frac{K_{1}\left(K_{2}-K_{1}\right)}{K_{1}^{2}-K_{2}} \\
\beta & =\frac{\left(1-K_{1}\right)\left(K_{2}-K_{1}\right)}{K_{1}^{2}-K_{2}} \\
K_{1} & =\frac{H_{r m s}^{2}}{H_{b}^{2}} \\
K_{2} & =\frac{H_{r m q}^{2}}{H_{b}^{4}} \\
H_{r m q} & =\text { Root-mean-quad wave height }
\end{aligned}
$$

This formulation is a three parameter distribution $\left(H_{b}, H_{r m s}\right.$, and $\left.H_{r m q}\right)$ and so should be an improvement over the one parameter Rayleigh distribution. Another improvement is that the distribution is bounded by a maximum wave height, whereas the Rayleigh distribution "tail" trails off into infinity. For deepwater waves, it is the tail region that shows the greatest deviation between theory and measured wave heights. Finally, the Beta-Rayleigh distribution transforms itself into the Rayleigh distribution when $H_{b}$ approaches infinity and $H_{r m q}=\sqrt{2} H_{r m s}^{2}$.

Comparison of measured wave heights with those predicted by the Beta-Rayleigh and Rayleigh distributions reveals that the new formulation is a significant improvement over the plain Rayleigh distribution. Also, empirical equations have been developed relating the three parameters of the distribution to the following common variables (Hughes and Borgman 1987):

$T_{p} \quad=$ peak spectral period,

$h \quad=$ water depth,

$H_{m o}=$ zeroth-moment wave height, equal to four times the standard deviation of sea surface elevations.

This thesis applies the Beta-Rayleigh distribution to particle velocities under irregular shallow water waves instead of wave heights. For the time series of 
horizontal velocities, the following one for one substitutions are made resulting in Equation 23.

$\begin{array}{lll}u & \text { for } & \eta, \\ u_{r m s} & \text { for } & \eta_{r m s}, \\ U / 2 & \text { for } & H \\ U_{r m s} / 2 & \text { for } & H_{r m s} \\ U_{r m q} / 2 & \text { for } & H_{r m q} \\ U_{m a x} / 2 & \text { for } & H_{m a x}\end{array}$

Similar substitutions are made for vertical velocities, $(w$ and $W$ ). The peak velocity is divided by 2 to account for the fact that peak particle velocities are measured from the mean velocity level to the maximum positive or negative velocity (crest or trough to mean level). Wave heights, $H$, are measured from the crest to the trough.

For the Rayleigh distribution, the following theoretical relationships hold (Hughes 1992, Hughes and Borgman 1987)

$$
\begin{aligned}
& U_{r m s}=\sqrt{2} u_{r m s} \\
& U_{r m q}=2 \sqrt{2} u_{r m s}^{2} .
\end{aligned}
$$

The following parameters can be determined from measurements using the relationships

$$
\begin{aligned}
U_{r m s} & =\left(\frac{1}{n} \sum_{i=1}^{n} U_{i}^{2}\right)^{\frac{1}{2}} \\
U_{r m q} & =\left(\frac{1}{n} \sum_{i=1}^{n} U_{i}^{4}\right)^{\frac{1}{2}} \\
U & =\text { peak velocity (either positive or negative direction) }
\end{aligned}
$$

Again, similar equations apply for vertical velocities.

Note that in studying peak velocity, one usually distinguishes between velocity in the positive and negative direction, unlike wave height that is defined by the total distance between peak and trough. 
The Beta-Rayleigh probability distribution function applicable to peak particle velocities is therefore

$$
P_{B R}(U)=\frac{2 \Gamma(\alpha+\beta)}{\Gamma(\alpha) \Gamma(\beta)} \frac{U^{2 \alpha-1}}{U_{m a x}^{2 \alpha}}\left(1-\frac{U^{2}}{U_{m a x}^{2}}\right)^{\beta-1}
$$

where

$$
\begin{aligned}
& \alpha=\frac{K_{1}\left(K_{2}-K_{1}\right)}{K_{1}^{2}-K_{2}} \\
& \beta=\frac{\left(1-K_{1}\right)\left(K_{2}-K_{1}\right)}{K_{1}^{2}-K_{2}}
\end{aligned}
$$

and

$$
\begin{aligned}
& K_{1}=\frac{U_{r m s}^{2}}{U_{m a x}^{2}} \\
& K_{2}=\frac{U_{r m q}^{2}}{U_{m a x}^{4}}
\end{aligned}
$$

with similar equations applying for vertical velocities.

\subsection{Experimental Results}

Practical application of the Beta-Rayleigh distribution can be done only if the three parameters of the distribution $\left(U_{m a x}, U_{r m s}\right.$, and $\left.U_{r m q}\right)$ are expressed in terms of common parameters of the wave field. For example, Hughes and Borgman (1987) developed empirical expressions relating $H_{b}, H_{r m s}$, and $H_{r m q}$ with $H_{m o}, T_{p}$, and $h$ for the case of shallow water irregular waves.

This investigation uses laboratory measurements to develop empirical relationships for shallow water irregular wave peak particle velocities. It will be shown that the empirical "fit" is very good. Unfortunately, the Beta-Rayleigh distribution provides only marginal improvement over the Rayleigh distribution.

Figure 24 plots measured and theoretical distributions for one of the time series (additional time series plots are in Appendix I, Figures 73 to 96). Figure 24 clearly shows the Beta-Rayleigh distribution being "pulled" in the direction of the peak of the measured velocity distribution. A hardcopy output from the computer program that calculated the statistics is in Figure 4. The measured and theoretical 


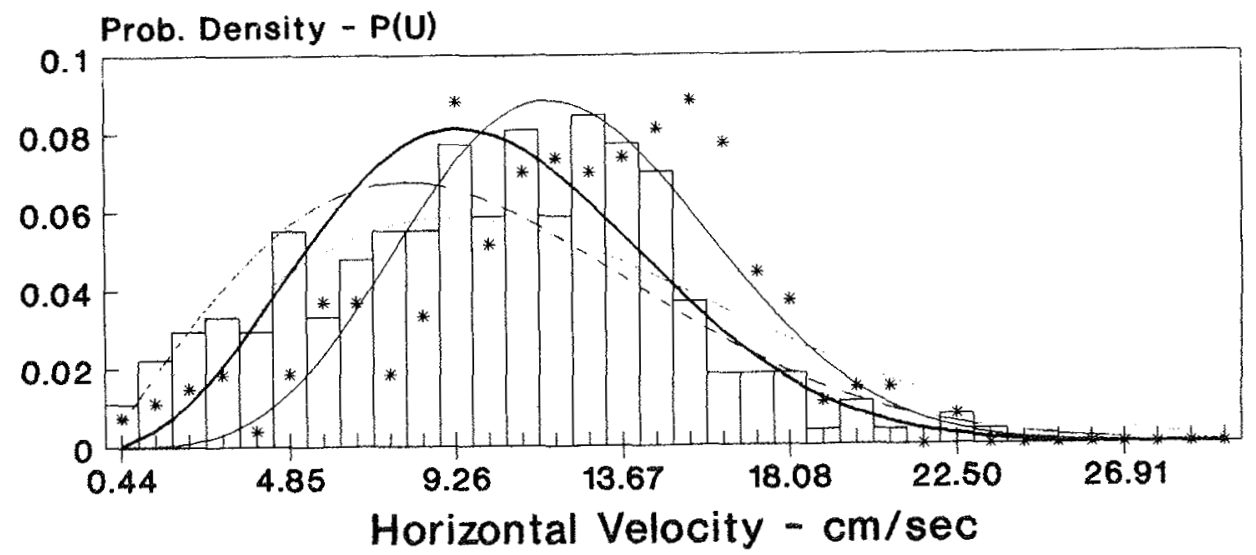

Measured Dist. (-) -..- Rayleigh Dist. (-) - Beta-Rayleigh (-)

* Measured Dist. (+) Raylolgh Dist. (+) - Beta-Raylelgh (+)

(a) Horizontal

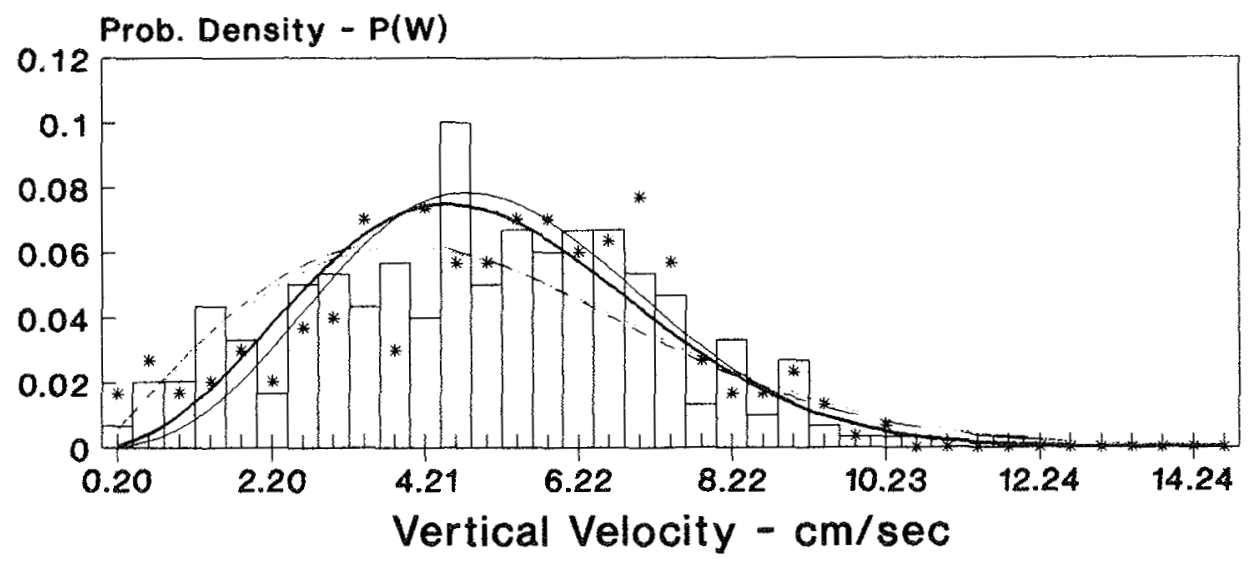

Measured Dist. (-) -... Rayleigh Dist. (-) - Beta-Rayleigh (-)

* Measured Dist. (+) Rayleigh Dist. (+) - Beta-Raylelgh (+)

(b) Vertical

Figure 24: Velocity Peaks Probability Distribution. (a) Horizontal; (b) Vertical 
Table 4: Beta-Rayleigh Distribution, Mean \%-RMS Error

\begin{tabular}{|l|r|r|r|r|r|r|}
\hline \hline & \multicolumn{3}{|c|}{ z=near bottom } & \multicolumn{3}{c|}{ z=mid-depth } \\
Direction & Rayleigh & Beta-R & Diff. & Rayleigh & Beta-R & Diff. \\
\hline Positive U & $40.5 \%$ & $39.3 \%$ & $1.2 \%$ & $43.4 \%$ & $42.0 \%$ & $1.4 \%$ \\
Negative U & $40.8 \%$ & $36.8 \%$ & $2.0 \%$ & $44.0 \%$ & $38.2 \%$ & $5.8 \%$ \\
Positive W & $56.1 \%$ & $44.4 \%$ & $11.7 \%$ & $42.8 \%$ & $37.3 \%$ & $5.5 \%$ \\
Negative W & $64.9 \%$ & $44.8 \%$ & $20.1 \%$ & $46.8 \%$ & $39.7 \%$ & $7.1 \%$ \\
\hline \hline
\end{tabular}

distributions tabulated in Figure 4 are an example of what was plotted for every time series.

Figures 25 and 26 plot the \%-RMS error for every time series as a function of $h / g T_{p}^{2}$. Additional plots are contained in Appendix I, Figures 56 to 58 (The use of \%-RMS error as a measure of "goodness-of-fit" is discussed in section 3.1). No significant trends are evident in the plots that would define when the BetaRayleigh distribution is an improvement over the Rayleigh distribution. However, on average the Beta-Rayleigh distribution is better than the Rayleigh distribution (Table 4). Table 4 lists the \%-RMS error for both distributions and for different velocity directions. The relative improvement is greater for near bottom vertical velocity than for near bottom horizontal velocity.

Filtering of the velocity time series was done before calculation of the theoretical and measured distributions. A band-pass filter in the range [0.20-5.00] seconds was selected based on comparison with the results of other filter widths (Figures 27,28 and in Appendix I, Figures 59 to 64). Figures 27 and 28 show that this filter range achieves the best agreement between the theoretical and measured distributions. However, a filter range of $[0.20-5.00] \mathrm{s}$ was not always optimal. Filtering can make a dramatic difference in data with high frequency turbulence and micro-eddies. Filtering also can improve a "noisy" signal caused by a poorly tuned LDV apparatus or other data acquisition imperfections. Fortunately, about half the time series looked almost as good as Figure $6 \mathrm{~b}$ prior to any filtering. Applying the filter to that relatively good signal will not change the down-crossing analysis results significantly. Therefore, in order to be consistent, a filter of $[0.20-5.00] \mathrm{s}$ was applied to all time series regardless of whether it was needed. Note that for turbulence studies it may be desirable to retain the effects of turbulent flow since turbulence is a real phenomena. However, this thesis assumes irrotational, inviscid flow for which turbulence does not theoretically exist.

Selection of bar width in plotting the measured distributions was a challenge. Ideally, one desires a constant bar width for ease of comparison between time series. 


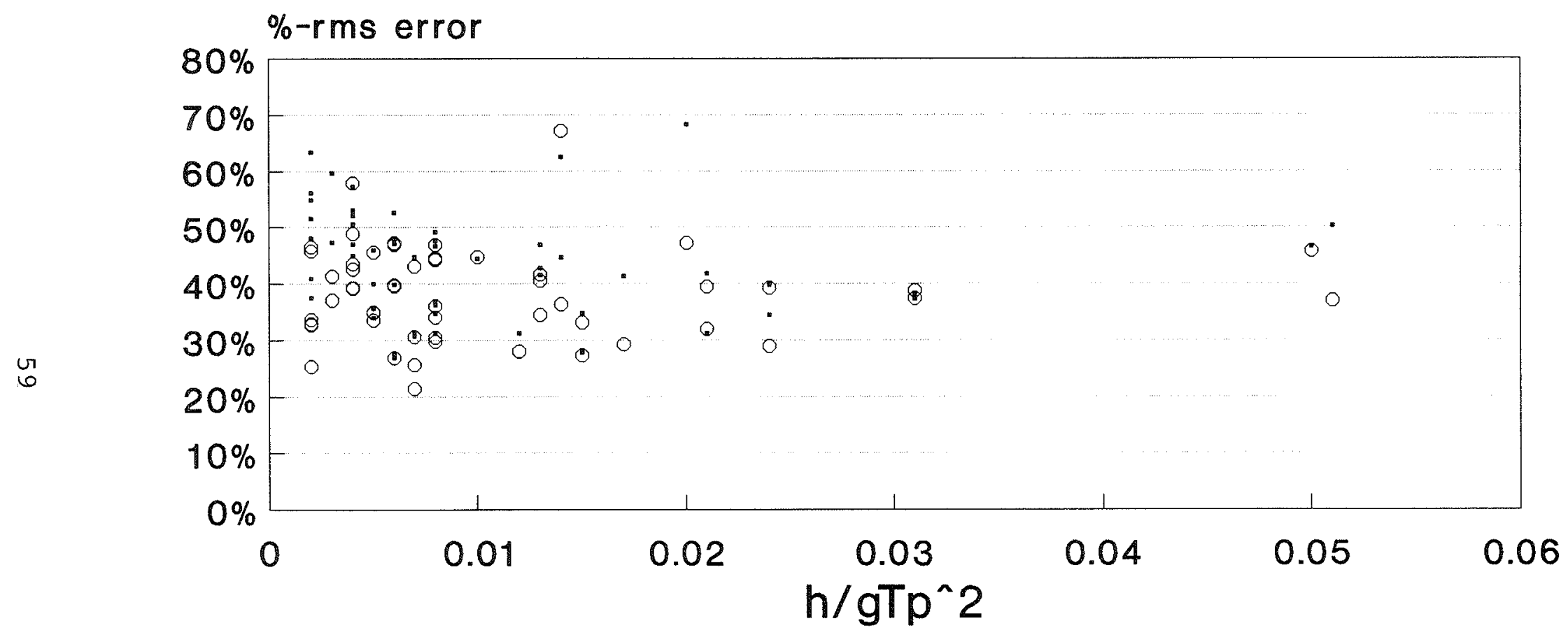

- Rayleigh,z=mid-depth $\quad$ Beta-R, $z=$ mid-depth

Figure 25: \%-Root-mean-square Error, Beta-Rayleigh Distribution and Rayleigh Distribution, Negative Horizontal Direction, Mid-depth 


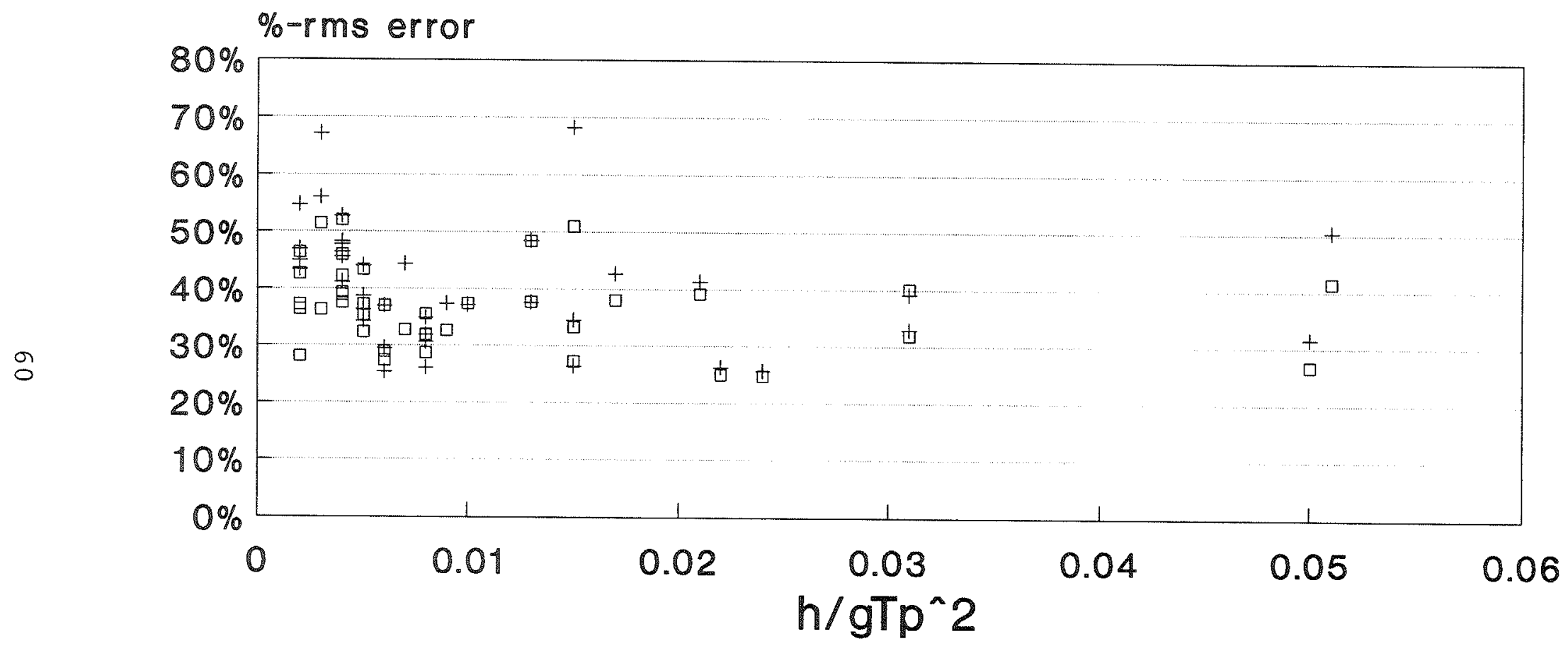

+ Rayleigh,z=bottom $\square$ Beta-R, $z=$ bottom

Figure 26: \%-Root-mean-square Error, Beta-Rayleigh Distribution and Rayleigh Distribution, Negative Horizontal Direction, Near Bottom 


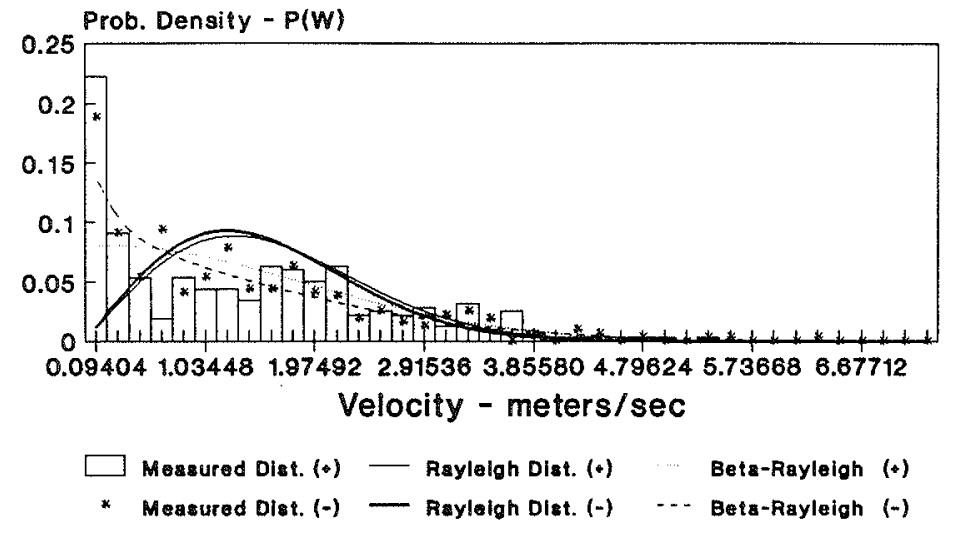

(a) Raw Data

ค

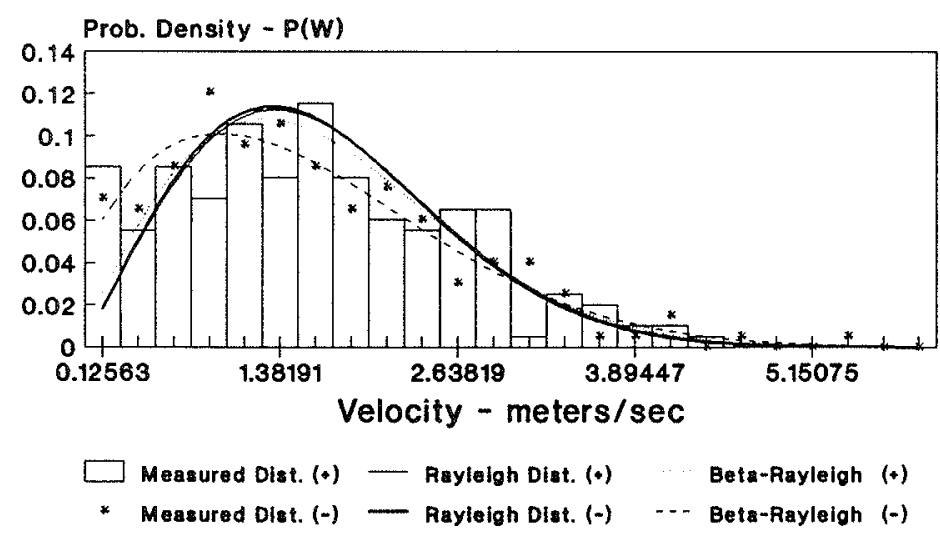

(c) Filtered Data [0.20-10.00] s

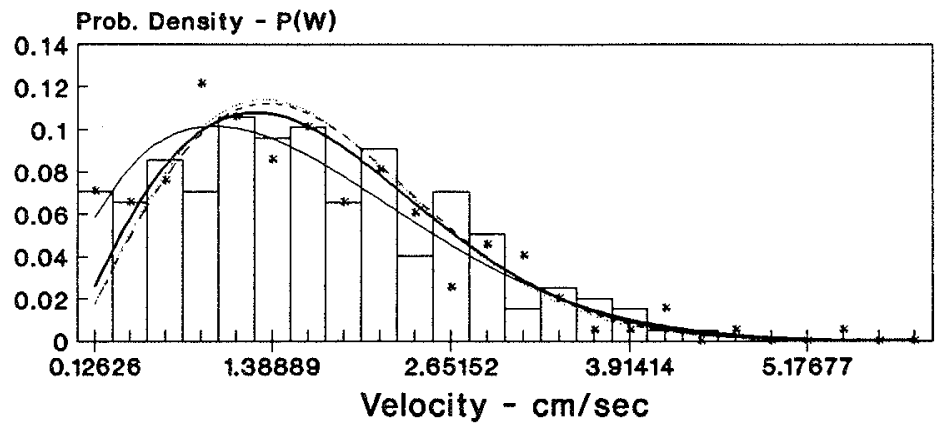

$$
\begin{aligned}
& \square \text { Measured Dis (. (+) -.. Rayleigh Dizt. (*) - Beta-Rayleigh (+) } \\
& \text { * Measured Dist. (-) Rayleigh Diet. (-) - Beta-Rayleigh (-) }
\end{aligned}
$$

(b) Filtered Data [0.20-5.00] s

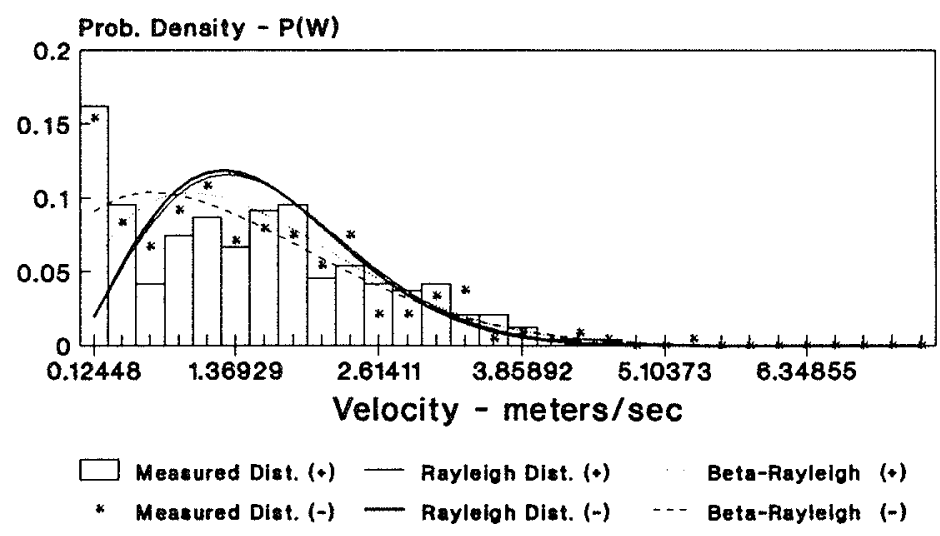

(d) Filtered Data [0.10-5.00] s

Figure 27: The Effect of Varying Filter Bandwidths on the Probability Distribution of Horizontal Velocity Peaks. (a) Raw Data; (b) Filtered Data [0.2-5.00] s; (c) Filtered Data [0.20-10.00] s; (d) Filtered Data [0.10-5.00] s 


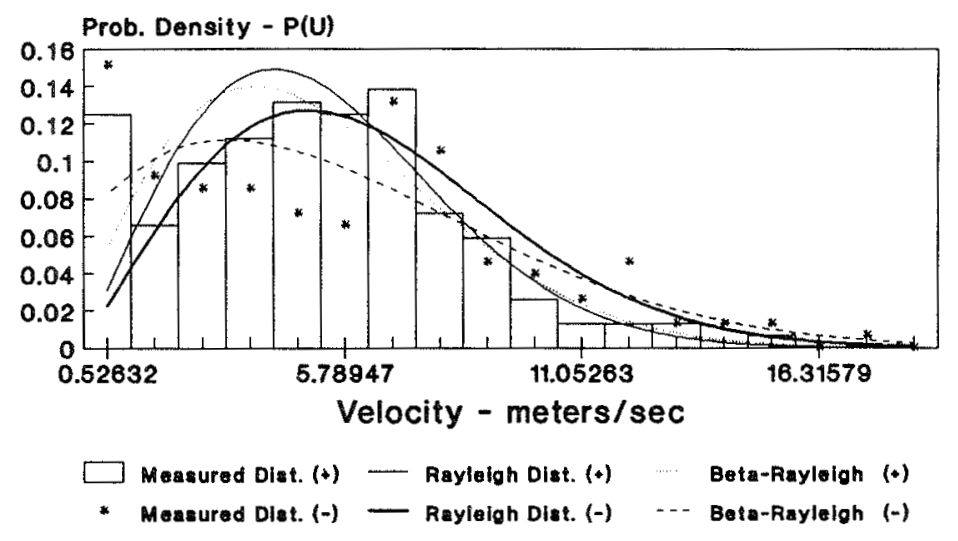

(a) Raw Data

$\stackrel{N}{N}$

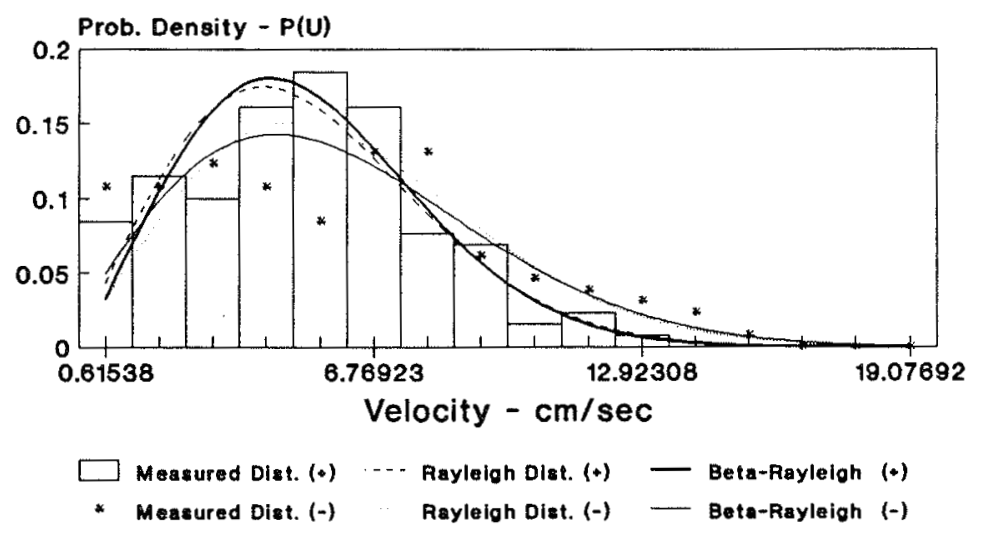

(b) Filtered Data [0.20-5.00] s

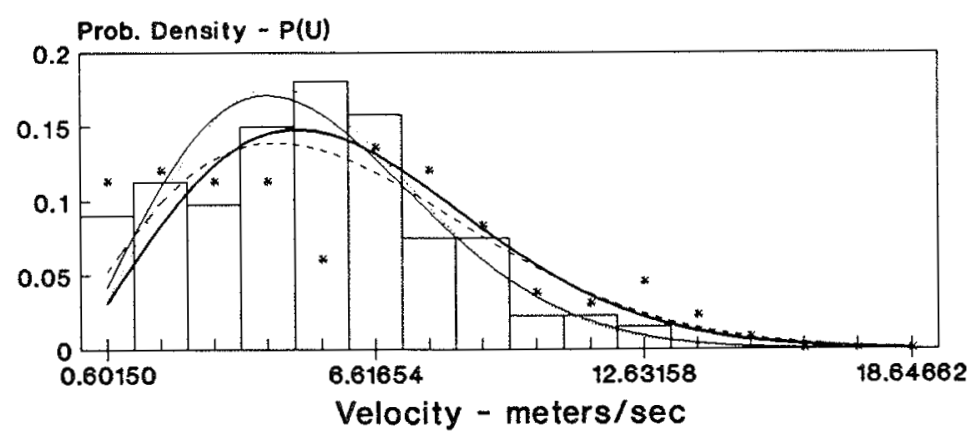

$\square$ Measured Dist. (+) — Rayleigh Dist. (o)

Beta-Rayleigh (•)

* Measured Dist. (-) - Rayleigh Dist. (-)

- Beta-Rayleigh (-)

(c) Filtered Data [0.20-10.00] s

(d) Filtered Data $[0.10-5.00] \mathrm{s}$

Figure 28: The Effect of Varying Filter Bandwidths on the Probability Distribution of Vertical Velocity Peaks: (a) Raw Data; (b) Filtered Data [0.20-5.00] s; (c) Filtered Data [0.20-10.00] s; (d) Filtered Data [0.10-5.00] s 
The plots of the velocity distribution for the entire time series have constant barwidth (Figures 19 and 20) because the large number of data points collected (8192 per time series) provide "stable" statistics that permit considerable flexibility in selecting bar width. However, the number of velocity peak data points measured per time series was 100 to 300 . This relatively low number of points is adequate for calculating stable statistics but barely adequate for graphing the measured data. Hence, a significant portion of the \%-RMS error calculated for every time series is attributed to these errors in the "discretization" of the theoretically smooth probability distribution.

\subsection{Parameterization}

An interesting way of comparing the Beta-Rayleigh to the Rayleigh distribution is found by plotting $U_{r m s} / u_{r m s}$ and $U_{r m q} / u_{r m s}^{2}$ vs. various non-dimensional parameters (Figures 29 and 30). These theoretical ratios, assuming the Rayleigh distribution (and its underlying assumptions) holds, are constants given by Equations 19 and 20. For the case of horizontal velocity there is a clear divergence from these theoretical ratios for increasing nonlinearities (indicated here by increasing Ursell number). One can conclude that horizontal velocity peaks are greater in the direction of wave propagation (in the positive direction) than in the direction opposite of wave travel (in the negative direction). No such difference is observed for the case of vertical velocity peaks. These observations agree with nonlinear second order Stokes theory that predicts higher horizontal velocities in the direction of wave travel (which is under the crest) than in the opposite direction (Dean and Dalrymple 1984). The results do not agree with previous investigations (Section 1.1). Note that linear wave theory predicts a symmetrical distribution of velocity. Quantification of this divergence may be worthwhile but is not directly relevant to this thesis.

Comparison of the Beta-Rayleigh and Rayleigh distributions is also done by plotting Beta-Rayleigh distributions with the parameters $U_{r m s}$ and $U_{r m q}$ calculated from Equations 19 and 20 (Figure 31 and in Appendix I, Figure 65). The effect is to create a two parameter distribution based on $u_{r m s}$ and $U_{m a x}\left(w_{r m s}\right.$ and $W_{m a x}$ in the vertical direction). Clearly, if $U_{\max }$ approaches infinity then this two parameter distribution should transition into the Rayleigh distribution. Also, there should be no difference between the positive direction and negative direction theoretical distributions since $u_{r m s}$ and $U_{m a x}$ apply to both directions. These features are evident in Figure 31 which shows the Beta-Rayleigh distribution nearly the same as the Rayleigh distribution. 


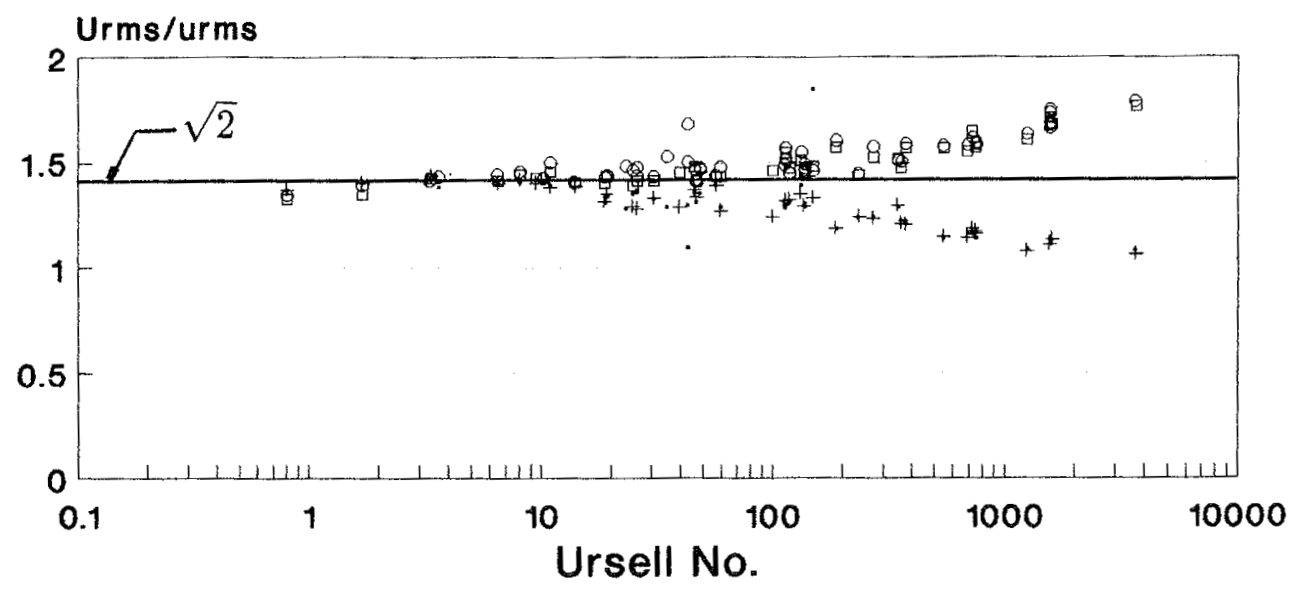

(a) $U_{r m s} / u_{r m s}$

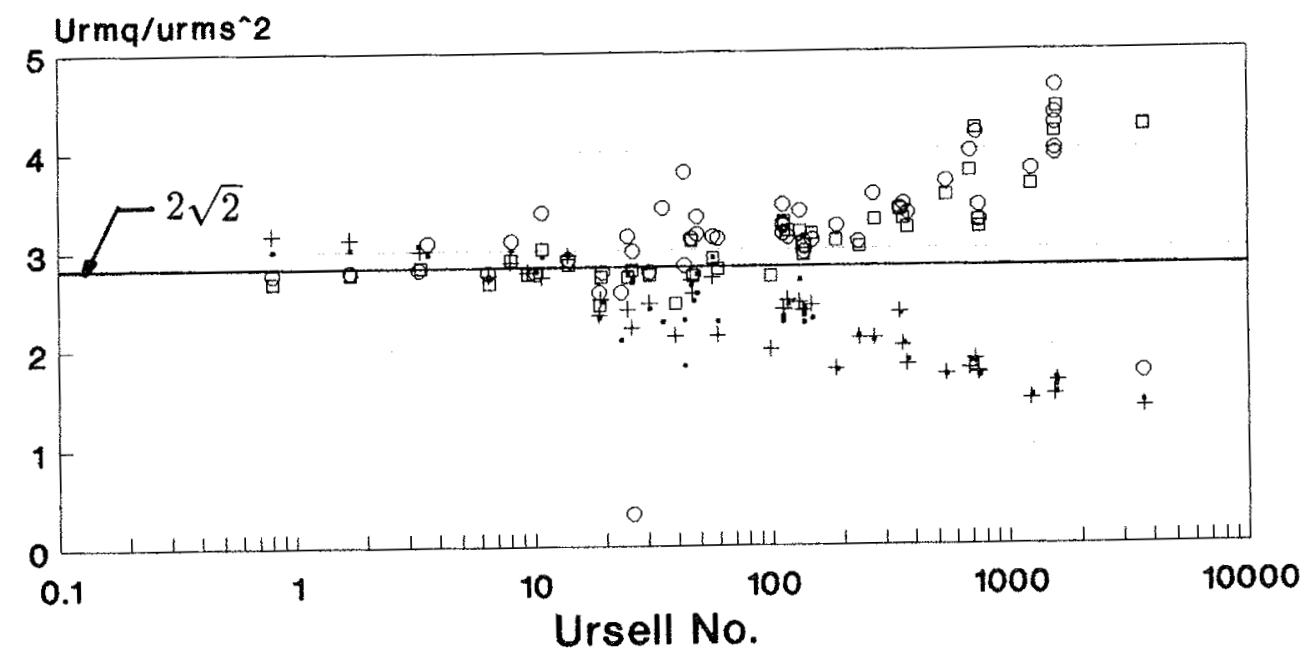

(b) $U_{r m q} / u_{r m s}^{2}$

$\begin{array}{ll}\text { V }(-), z \circ \text { mid-depth } & +\quad U(-), z \text { bottom } \\ 0 \quad U(+), z \circ \text { mid-depth } & \square \quad U(+), z \text { bottom }\end{array}$

Figure 29: Theoretical Ratios, Beta-Rayleigh Distribution, Horizontal Direction. (a) $U_{r m s} / u_{r m s} ;$ (b) $U_{r m q} / u_{r m s}^{2}$ 


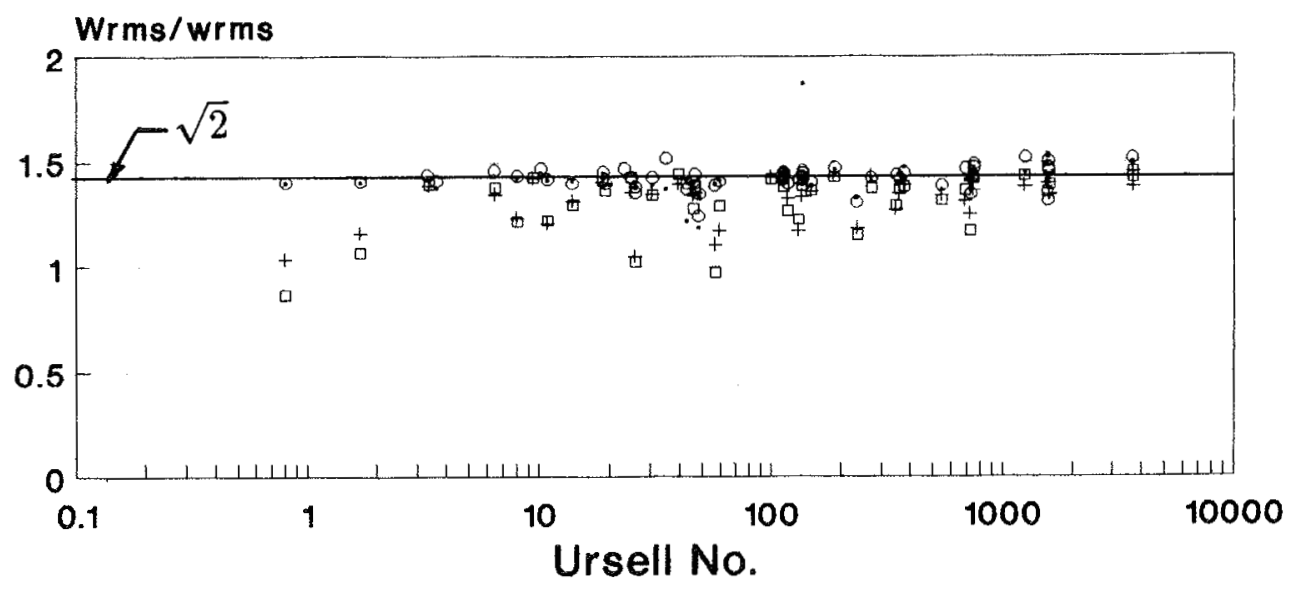

(a) $W_{r m s} / w_{r m s}$

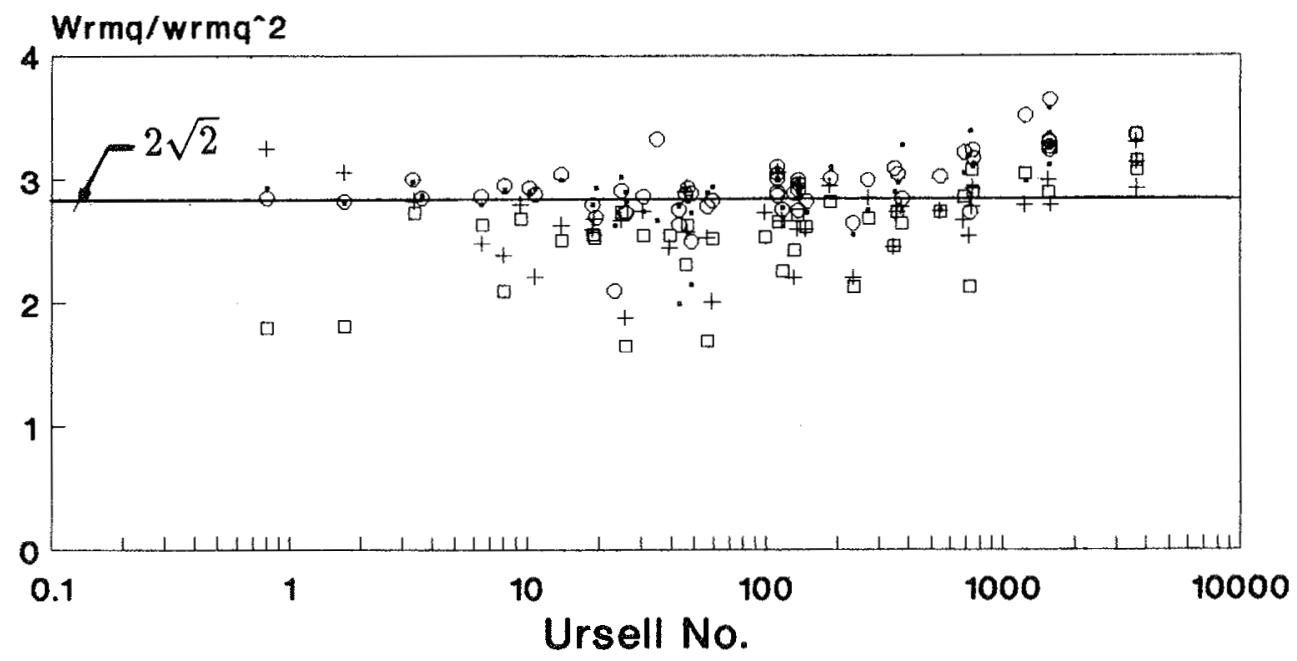

(b) $W_{r m q} / w_{r m s}^{2}$

$\begin{array}{ll}W(+), z \bullet \text { mid-depth } & +W(+), z \bullet \text { bottom } \\ W(-), z \bullet \text { mid-depth } & \square W(-), z \bullet \text { bottom }\end{array}$

Figure 30: Theoretical Ratios, Beta-Rayleigh Distribution, Vertical Direction. (a) $W_{r m s} / w_{r m s} ;$ (b) $W_{r m q} / w_{r m s}^{2}$ 


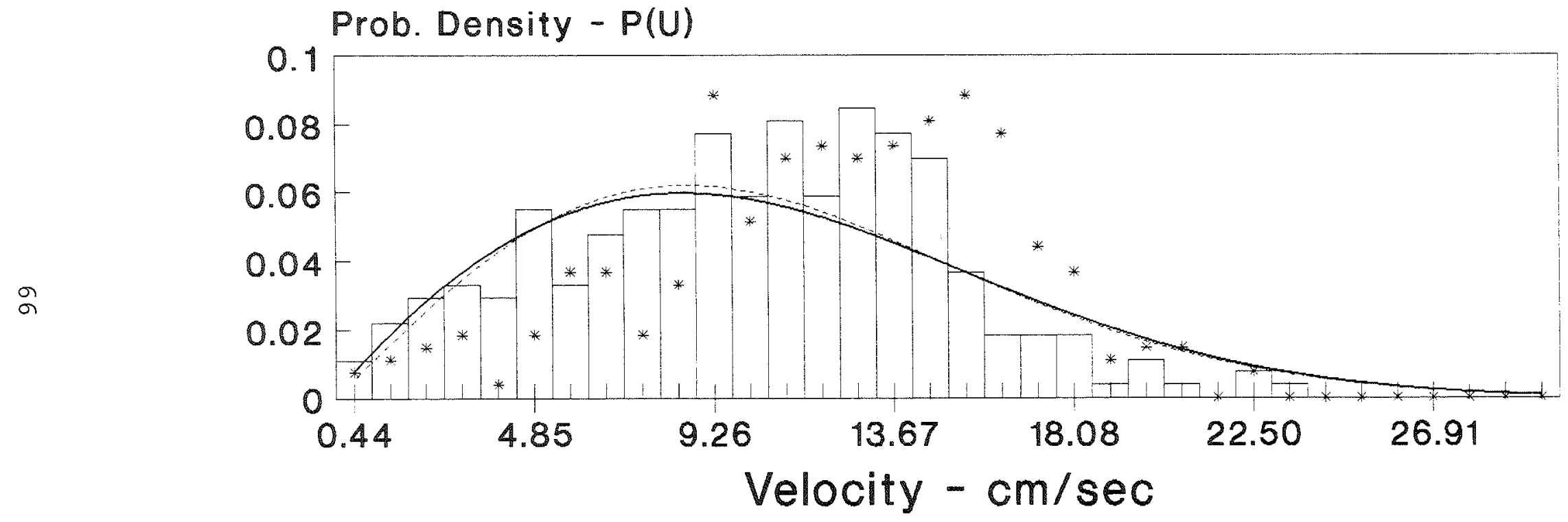

$\begin{array}{llll}\square & \text { Measured Dist. (-) } & \text { Rayleigh Dist. (-) } & - \text { Beta-Rayleigh (-) } \\ \text { * Measured Dist. (+) } & \text { Rayleigh Dist. (+) } & - \text { Beta-Rayleigh (+) }\end{array}$

Figure 31: Horizontal Velocity Peaks Probability Distribution, $u_{r m s}$ Only Parameter 


\subsubsection{Maximum Velocity}

The development of an expression for maximum velocity is more difficult than determination of a breaking wave height. Many investigations both theoretical and empirical have been made into the subject of breaking wave height. The simple expression

$$
H_{b}=0.78 h
$$

where $h=$ water depth is widely used (McCowan 1894).

The Shore Protection Manual (1984) suggests the following expression for the maximum horizontal velocity under a breaking wave

$$
U_{\max }=\sqrt{g(H+h)}
$$

where $(H+h)$ is the distance measured from the crest of the breaker to the bottom.

Equation 29 is based on laboratory measurements by Daily and Stephan (1953) for solitary waves. The expression is probably more applicable to wave action in the surf zone where a broken wave moves forward as a bore of water. This thesis is more concerned with fluid particle velocities under progressive waves where wave breaking is governed more by wave steepness than by limited depth.

For a shallow water regular wave, linear wave theory predicts the following horizontal and vertical velocities (Shore Protection Manual 1984)

$$
\begin{aligned}
u & =\frac{H}{2} \sqrt{\frac{g}{h}} \cos \theta \\
w & =\frac{H \pi}{T}\left(1+\frac{z}{h}\right) \sin \theta
\end{aligned}
$$

where shallow water is defined as

$$
\frac{h}{L}<\frac{1}{25}
$$

$\theta$ is the phase of the regular wave and $z$ is positive upwards measured from the stillwater level. These expressions are derived from Equations 2 and :3

Expressions for the maximum velocities can be developed by as:uming that $H_{\max }=h$ (an assumption based on Equation 28). Also consider that the maximum velocity will occur when $\cos \theta=1.0$. Finally, a reasonable choice for $T$ in Equation 31 is $T_{p}$. Making these substitutions one arrives at the following expressions for 
the maximum horizontal and vertical velocity

$$
\begin{aligned}
U_{\max } & =\frac{\sqrt{g h}}{2} \\
W_{\max } & =\frac{h \pi}{T_{p}}\left(1+\frac{z}{h}\right) .
\end{aligned}
$$

A change is made to Equation 34 in applying it to this investigation. The mean period $(\bar{T})$ of the velocity time series is used instead of the peak period $\left(T_{p}\right)$ measured from the water elevation time series. Other than this, Equations 33 and 34 are identical to those applied in this investigation. This change was made in order to simplify the calculation procedure. This way all the statistics of the velocity time series could be calculated independent of the water elevation statistics. The mean period of velocity is generally less than the peak period of wave heights, at times $50 \%$ less than $T_{p}$. As will be shown below, the effect on the resulting theoretical distributions is not significant.

Comparison of measured with theoretical maximum velocity is made in Figure 32 and in Appendix I, Figure 66. A clear trend is evident whereby the ratio of measured to predicted maximum velocity increases with $H_{m o} / h$. This trend is interesting but is not applied in this thesis. Figure 33 and in Appendix I, Figures 67 and 68 show that a precise determination of $U_{\max }$ and $W_{\max }$ is not necessary in applying the Beta-Rayleigh distribution to velocities. The difference in the theoretical distributions for $0.5 \times$ and $2.0 \times$ the values in Equations 33 and 34 is barely discernible. A value less than $0.5 \times$ the usual value of $U_{\max }$ and $W_{\max }$ would be hard to justify in physical terms and would be past the limit of measured values (Figure 32). Additional research is probably worthwhile on the general subject of "extremal" velocity estimates.

The assumption of "shallow water" as defined by Equation 32 deserves closer scrutiny. Equation 33 is independent of $z$ and this is correct in a physical sense for shallow water linear waves. However, some components of the wave spectrum probably do not satisfy Equation 32 and therefore some decrease in $U_{\text {max }}$ with increasing depth below water surface can be expected. Unfortunately, the experimental program was not intended to investigate fully the variation of velocities with $z$ and therefore it is difficult to quantify the relationships. Half the measurements were taken at mid-depth $(z / h=0.5)$ and half at "near bottom" $(z / h=0.85$ to 0.94 ). As explained in the above paragraph, a precise determination of $U_{\max }$ is not important in applying the Beta-Rayligh distribution.

Several clues are available which show that the shallow water assumption of $u$ being independent of $z$ is reasonable. First, Figure 32 plots the ratio of mea- 


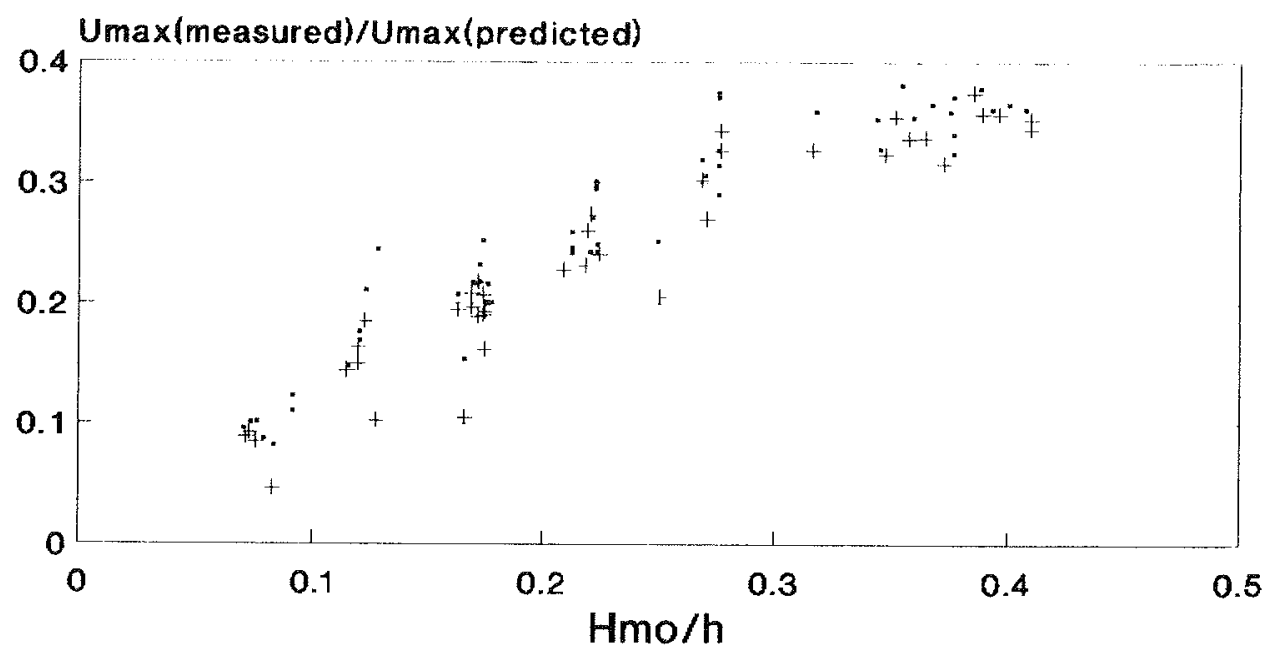

- $U, z$ mid-depth $\quad+\quad U, z$ near bottom

(a) Horizontal Direction

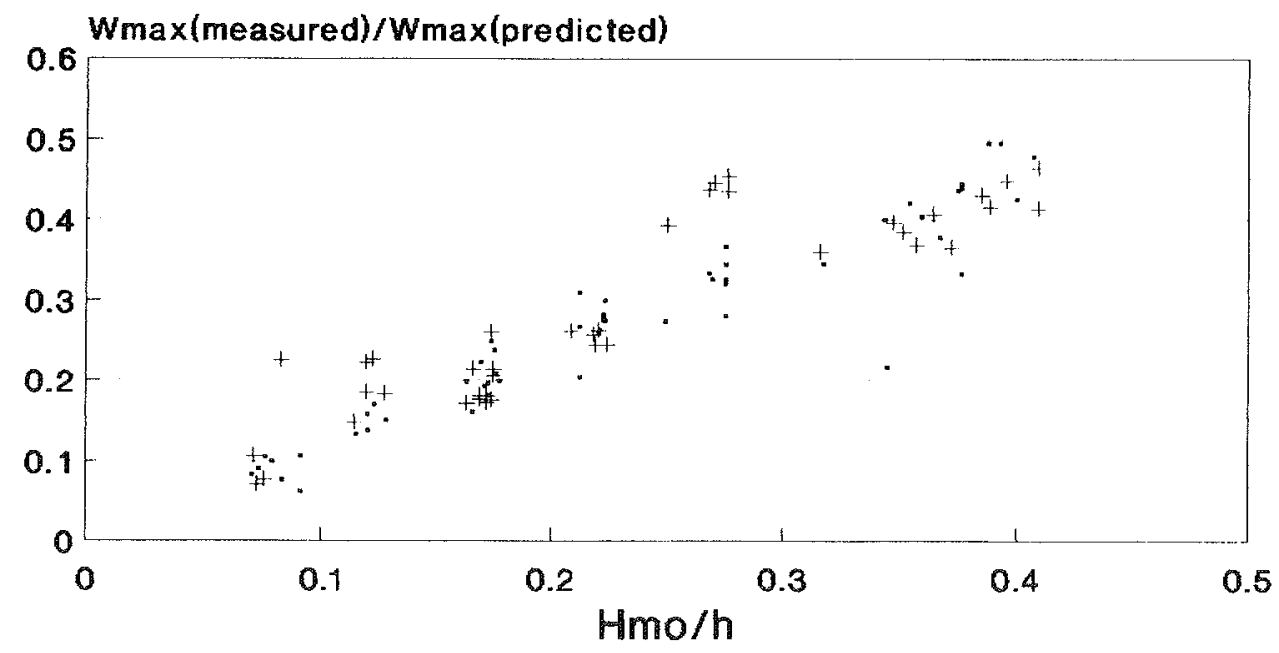

- W, $z$ mid-depth $+W, z$ near bottom

(b) Vertical Direction

Figure 32: Maximum Velocity Measured/Predicted. (a) Horizontal Direction; (b) Vertical Direction 


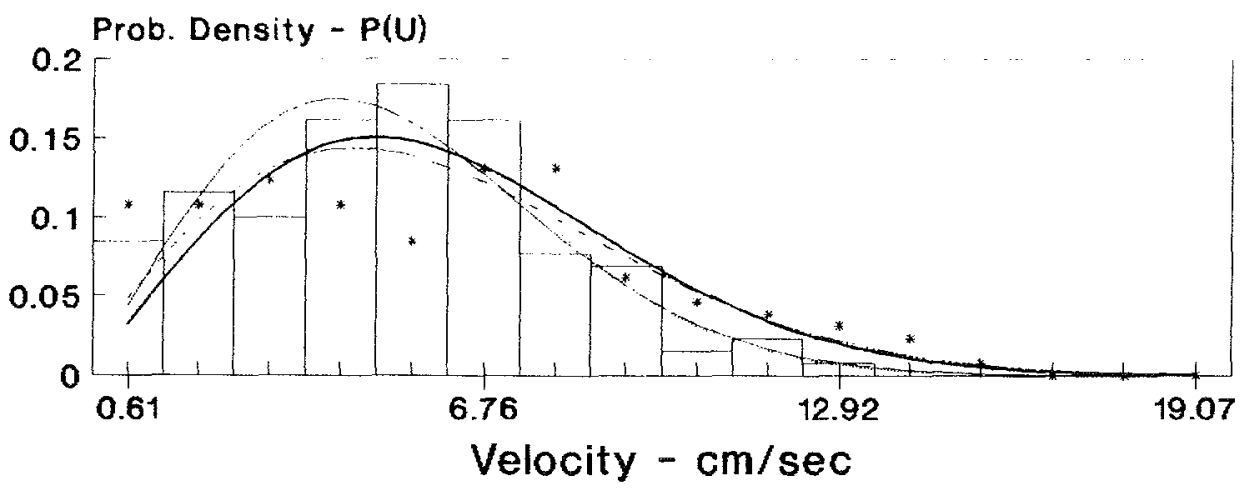

$\left.L^{-}\right]$Measured Dist. (-) - Rayleigh Dist. (-) Beta-Rayleigh (-)

* Measured Dist. (*) - Rayleigh Dist. (*) Beta-Rayleigh $(*)$

(a) $2 \mathrm{x}$ Usual $U_{\max }$

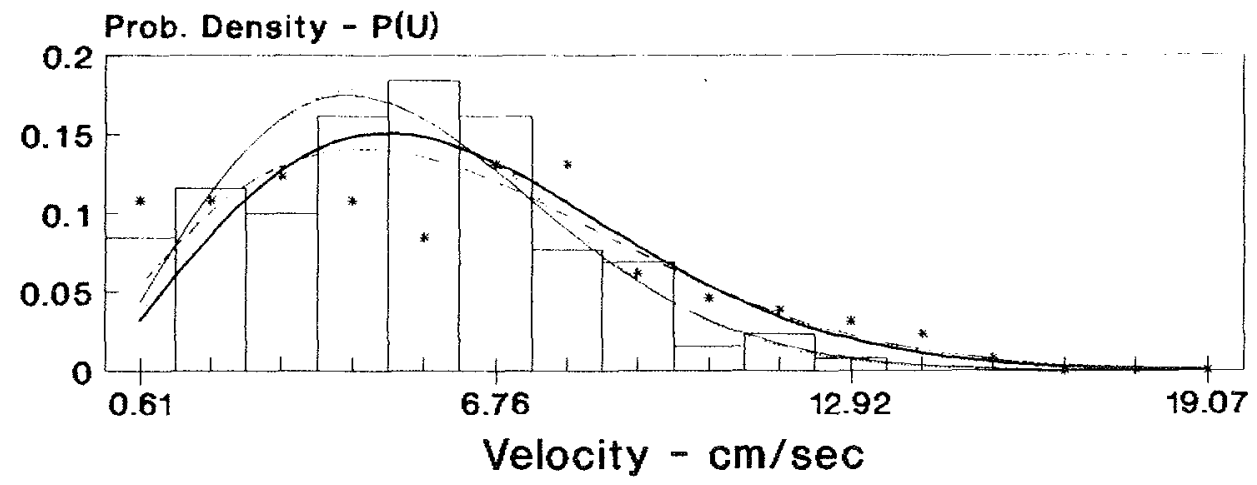

Jeasured Dist. (-) - Rayleigh Dist. (-) Beta-Rayleigh (-)

* Measured Dist. (+) - Rayleigh Dist. (+) … Beta-Rayleigh (+)

(b) $0.5 \mathrm{x}$ Usual $U_{\max }$

Figure 33: Horizontal Velocity Peaks Probability Distribution. (a) $2 x$ Usual $U_{\max }$; (b) $0.5 x$ Usual $U_{\max }$ 
sured/predicted $U_{\max }$. No large difference is evident between mid-depth and near bottom measurements, predicted $U_{\max }$ is nearly the same for both depths. One would expect mid-depth ratios to be greater if $u$ were a function of $z$ (the same wave conditions were run twice, one at mid-depth and one near bottom).

A second indication is provided by Figure 34, which plots the mean peak horizontal velocity $(\bar{U})$ in the positive and negative directions for every time series. Again, one would expect the mid-depth horizontal velocities to be higher than the near bottom velocities if the shallow water assumption is not valid. Here, the middepth $\bar{U}$ is larger than the near bottom $\bar{U}$, but only slightly. A third indication is provided by Figure 35 (discussed in the next section). Again, there is no practical difference between near bottom and mid-depth horizontal velocity measurements.

The maximum vertical velocity, $W_{\max }$, predicted by Equation 34 is a function of $z$. However, the predicted values of $W_{\max }$ are also affected by the shallow water approximation in linear theory. The effect is just not as clear as that for horizontal velocity where $z$ drops out completely. The discrepancy due to just the shallow water approximation is difficult to quantify for $w$. This discussion of the shallow water approximation is part of a more general concern regarding the range of applicability of the equations in this thesis (ranges of applicability are defined in the next section).

\subsubsection{Root-Mean-Square and Root-Mean-Quad Peak Velocity}

Nondimensional plots of the Root-mean-square and Root-mean-quad peak velocities vs. relative depth are presented in Figures 35 and 36. Additional plots using other nondimensional parameters are in Appendix I, Figures 69 to 72. Clear trends are evident which lend themselves to empirical curve fitting. Regression analysis yields the following equations

$$
\begin{aligned}
& \frac{U_{r m s} T_{p}}{H_{m o}}(+)=0.006028(x)^{-1.9830} \exp \left[-0.14391 \cdot \ln (x)^{2}\right], \frac{z}{h}<0.5 \\
& \frac{U_{r m s} T_{p}}{H_{m o}}(-)=0.010565(x)^{-1.6598} \exp \left[-0.09583 \cdot \ln (x)^{2}\right], \frac{z}{h}<0.5 \\
& \frac{U_{r m q} T_{p}^{2}}{H_{m o}^{2}}(+)=0.000057(x)^{-3.9741} \exp \left[-0.29475 \cdot \ln (x)^{2}\right], \frac{z}{h}<0.5 \\
& \frac{U_{r m q} T_{p}^{2}}{H_{m o}^{2}}(-)=0.000229(x)^{-3.1383} \exp \left[-0.17244 \cdot \ln (x)^{2}\right], \frac{z}{h}<0.5
\end{aligned}
$$




$$
\begin{aligned}
& \frac{W_{r m s} T_{p}}{H_{m o}}=0.178386(x)^{-0.4841} \exp \left[-0.01617 \cdot \ln (x)^{2}\right], \frac{z}{h}=0.5 \\
& \frac{W_{r m s} T_{p}}{H_{m o}}=0.029270(x)^{-0.5147} \exp \left[-0.00083 \cdot \ln (x)^{2}\right], 0.80<\frac{z}{h}<0.95 \\
& \frac{W_{r m q} T_{p}^{2}}{H_{m o}^{2}}=0.062737(x)^{-0.7946} \exp \left[-0.01030 \cdot \ln (x)^{2}\right], \frac{z}{h}=0.5 \\
& \frac{W_{r m q} T_{p}^{2}}{H_{m o}^{2}}=0.011076(x)^{-0.0928} \exp \left[-0.09545 \cdot \ln (x)^{2}\right], 0.80<\frac{z}{h}<0.95
\end{aligned}
$$

where

$$
x=\frac{h}{g T_{p}^{2}}
$$

all valid only in the range

$$
0.002<\frac{h}{g T_{p}^{2}}<0.05 .
$$

The positive horizontal direction is defined as being in the direction of wave travel. The negative horizontal direction is opposite the direction of wave travel. The positive vertical direction is upwards.

Each equation is of the form

$$
y=a(x)^{b} \exp \left[c \cdot \ln (x)^{2}\right] .
$$

Reasonable upper and lower limits to the observed data are defined by replacing " $a$ " in Equations 35 through 42 with the values in Table 5. Included in Table 5 are the correlation coefficients for the above equations.

An interesting phenomenon is that for horizontal velocities (Figure 35), the mid-depth and near bottom measured data coincide, while the positive and negative directions diverge for increasing nonlinearities. For vertical velocities (Figure 36), the mid-depth and near bottom measured data are different (including increased divergence with increasing nonlinearities) while the positive and negative directions coincide. These observations agree with linear wave theory.

In principle, it would be desirable to combine Equations 39 and 40 as well as Equations 41 and 42 into single equations that are a function of $z$. Unfortunately, the data set only includes points at mid-depth and near bottom making development of such equations difficult. The author recommends applying linear 
Table 5: Root-Mean-Square and Root-Mean-Quad Curve Fitting

\begin{tabular}{|c|c|c|c|}
\hline \hline $\begin{array}{c}\text { Equation } \\
\text { Number }\end{array}$ & $\begin{array}{c}\text { Correlation } \\
\text { Coefficient }\end{array}$ & $\begin{array}{c}\mathrm{a} \\
\text { upper limit }\end{array}$ & $\begin{array}{c}\mathrm{a} \\
\text { lower limit }\end{array}$ \\
\hline 35 & 0.975 & 0.007447 & 0.004992 \\
\hline 36 & 0.987 & 0.012916 & 0.008651 \\
\hline 37 & 0.973 & 0.000075 & 0.000045 \\
\hline 38 & 0.973 & 0.000335 & 0.000203 \\
\hline 39 & 0.942 & 0.223130 & 0.135335 \\
\hline 40 & 0.817 & 0.049787 & 0.018316 \\
\hline 41 & 0.949 & 0.082085 & 0.040762 \\
\hline 42 & 0.832 & 0.030197 & 0.004087 \\
\hline \hline
\end{tabular}

interpolation for values of $z / h$ between 0.80 and 0.50 (In principle the interpolation should be a parabolic function to match linear wave theory. However, given the scatter in the measured data, the error in using linear interpolation should be insignificant.) For values of $z / h$ between 0.95 and 0.80 , use of Equations 40 and 42 only is recommended. Application of these equations above $z / h=0.5$ or below $z / h=0.95$ is not recommended. 


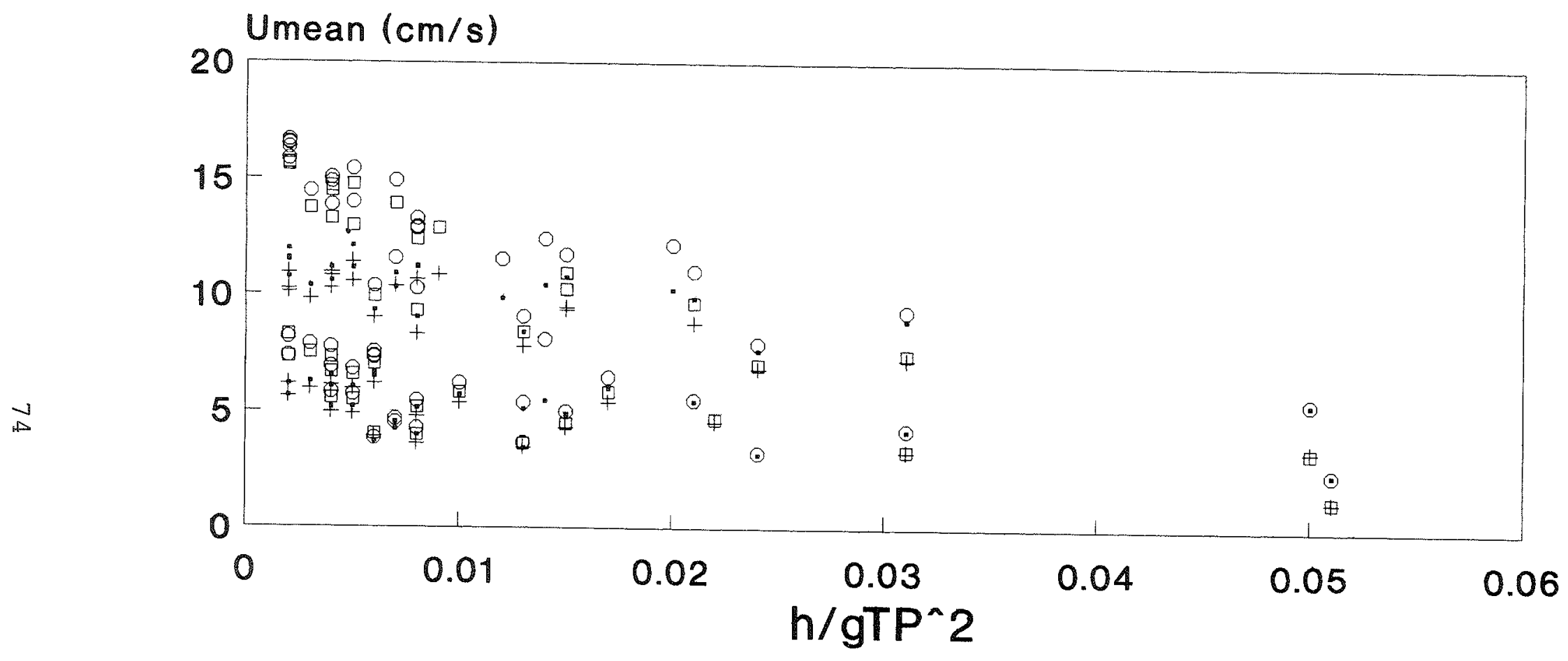

- $\quad U,(-)$, mid-depth

- $U,(+)$, mid-depth $+\quad U,(-)$, near bottom
$\square \quad U,(+)$, near bottom

Figure 34: Mean Horizontal Velocity Peaks 


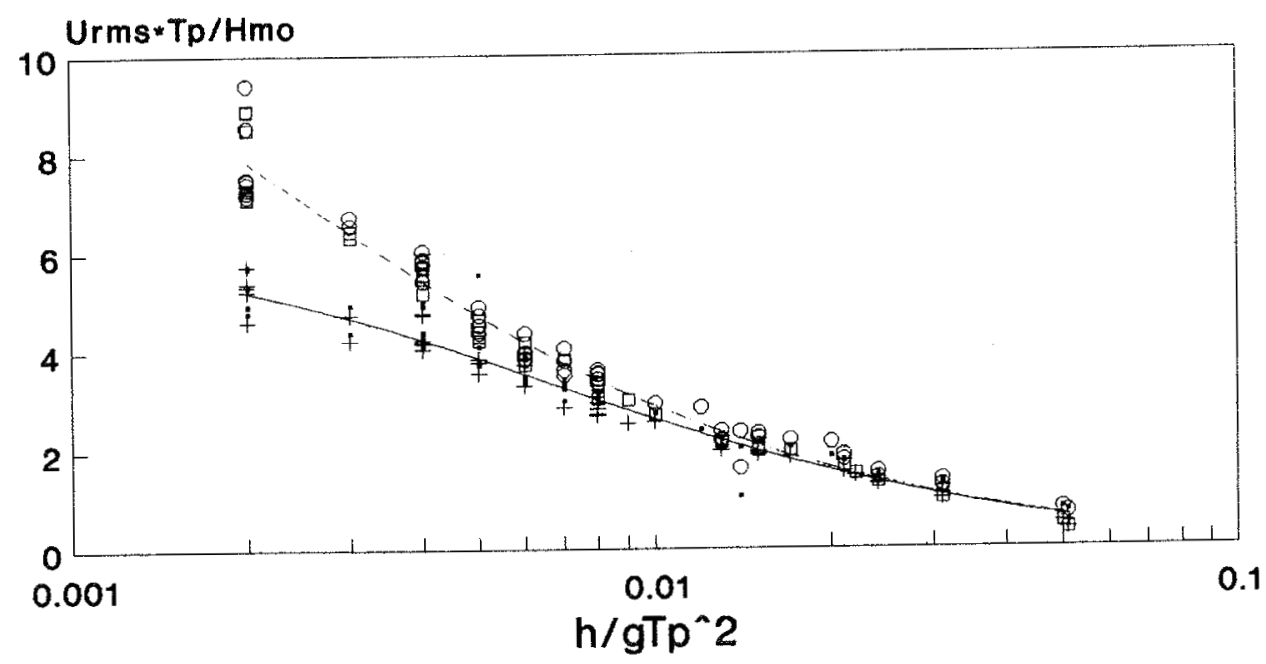

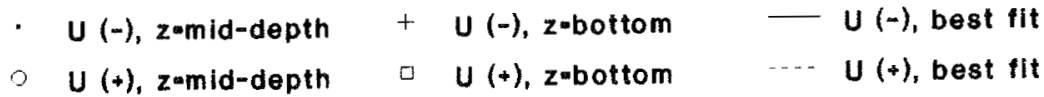

(a) $U_{r m s}$

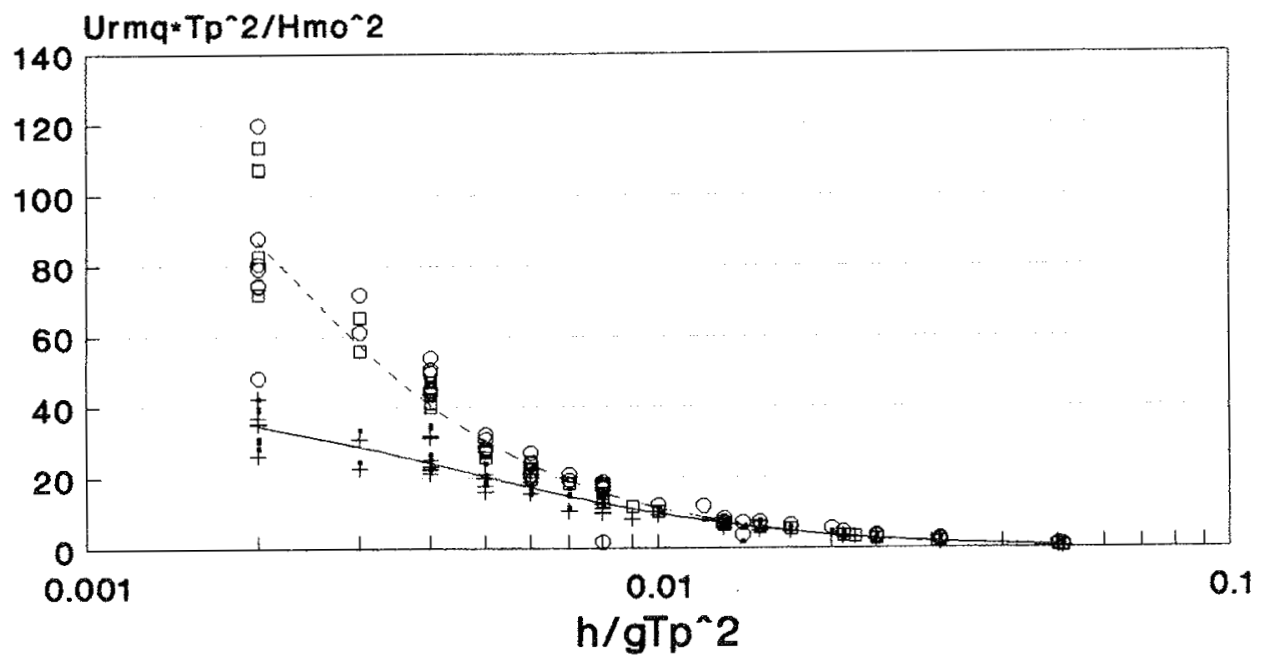

$-U(-), z=$ mid-depth
$0 \quad U(+), z=$ mid-depth $\quad \square \quad U(-), z$, $z$-bottom $\quad-U(-)$, best fit
$0 \quad \cdots(+)$, best fit

(b) $U_{r m q}$

Figure 35: Parameterization of Beta-Rayleigh Distribution, Horizontal Direction. (a) $U_{r m s} ;$ (b) $U_{r m q}$ 


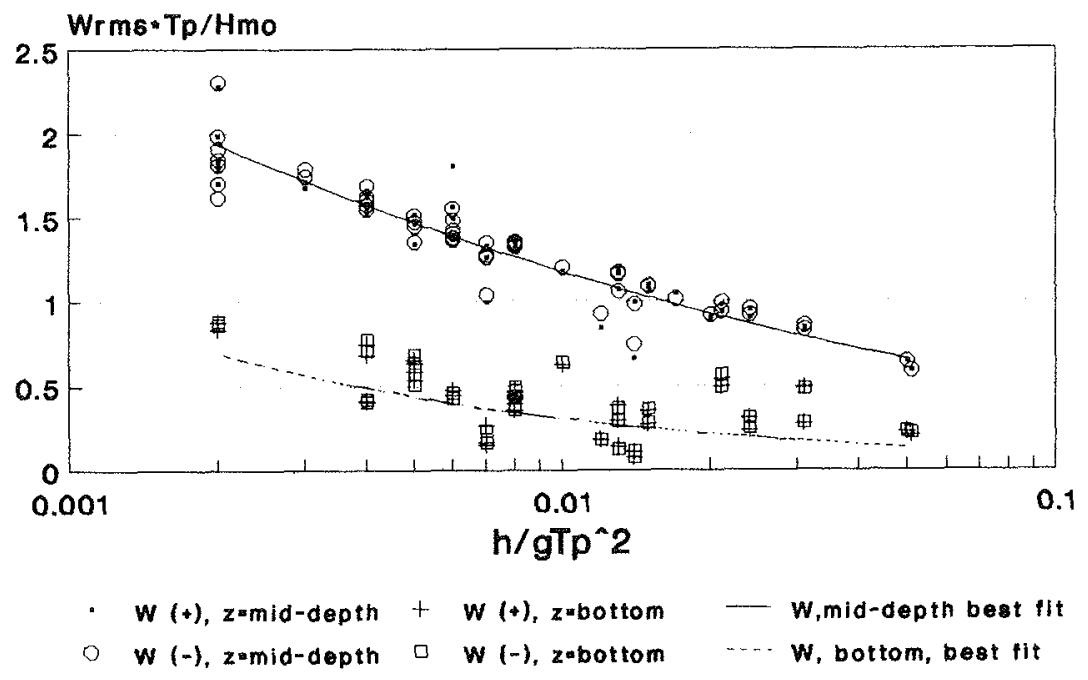

(a) $W_{r m s}$

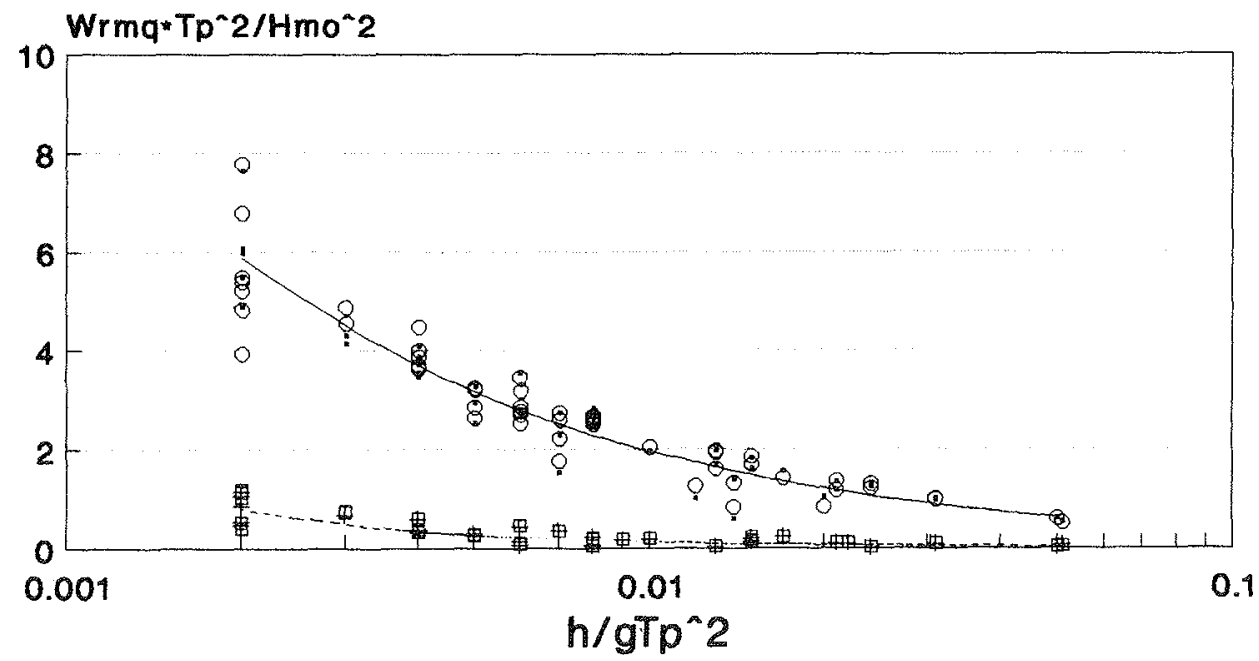
- W (\$), zmmid-depth
$+\quad W(+), z$ abottom
W,mid-depth best ilt
o W (-), zamld-depth
口 W (-), zabottom
W, bottom, best fit

(b) $W_{r m q}$

Figure 36: Parameterization of Beta-Rayleigh Distribution, Vertical Direction. (a) $W_{r m s} ;$ (b) $W_{r m q}$ 


\section{SUMMARY AND CONCLUSIONS}

In this study, irregular wave shallow water wave particle velocities were investigated with a laser Doppler velocimeter (LDV). Measurements were made in a flat bottom 2-dimensional wave tank with a spending beach at one end. Of special interest is the effect of nonlinear wave conditions on certain theoretical and empirical expressions. Both the horizontal and vertical components of velocity are analysed. Surprisingly good results are obtained for vertical velocities, even for measurements near the bottom where one would expect difficulty in recording the relatively small velocity fluctuations.

An array of three surface piercing capacitance type wave gauges was placed in the middle of the tank to resolve incident and reflected wave height spectra using the method of Goda and Suzuki (1976). This method is itself based on linear superposition of individual wave components. However, the reflection coefficients were on the order of $5-15 \%$ thereby minimizing the importance of a precise resolution of incident and reflected spectra.

Before the irregular wave time series, regular waves were run in order to optimize the design of the wave absorbing beach. It was discovered that the reflection coefficient varies directly with the ratio wave height/depth. Surprisingly, the amount of wave reflection was not correlated with peak spectral period.

A total of 108 irregular wave time series were run. The water depth, peak spectral period, and significant wave height were varied to provide a range of wave conditions. LDV measurements were made at mid-depth and at $3 \mathrm{~cm}$ above the bottom. Each time series was run for approximately $5 \mathrm{~min}$. Collection of velocity data with the LDV and water elevation data with the capacitance gages was not synoptic. Instead, statistics based on measured velocity data were compared with theoretical statistics based on the measured water elevations.

One objective of this study is the investigation of theoretical expressions for the root-mean-square horizontal and vertical velocity (Equations 4 and 5). The rms velocity is a useful and important parameter in studies of sediment transport and turbulence. Results indicate that predictions within $10 \%$ are possible given the components of the incident wave height spectrum, the reflection coefficients, and the water depth. Empirical corrections to Equations 4 and 5 are not developed because of the lack of clear trends between the measured and theoretical values. One would expect only a small deviation between predicted and measured $u_{r m s}$ and $w_{r m s}$ due to nonlinearities. This is due to the fact that while the nonlinear wave profile is different, the value of $\eta_{r m s}$ is only slightly different. The same effect 
would extend to velocities.

In applying Equations 4 and 5 this investigation discovered that there is no difference between using a random phase angle $\left(\theta_{n}\right)$ and a measured phase angle. A measured phase angle results from the Fourier analysis of the wave signal. A random phase angle is a random value between $0^{\circ}$ and $90^{\circ}$ applied in Equations 4 and 5 to predict $u_{r m s}$ and $w_{r m s}$. Also, the range of variability of the rms velocities with varying distance from the toe of slope $(x)$ is less than $0.05 \%$ for horizontal distances more than $1 \mathrm{~m}$ from the reflecting structure (This result may not be applicable to structures with reflection coefficients greater than $20 \%$ ). Therefore, it is concluded that the value of $x$ in Equations 4 and 5 should be simply the distance from the toe of slope.

Also studied is the application of an extended form of the Gaussian probability distribution function to wave particle velocities (Equation 6). That is, the distribution of all velocity measurements, not just peak velocities. This Gram-Charlier distribution is in the form of a series using Hermite polynomials and makes use of higher moments of the distribution like skewness and kurtosis. It is concluded that the theoretical Gram-Charlier distribution is less effective than the plain Gaussian distribution in fitting the measured data.

Analysis of peak velocities reveals that a modified form of the Rayleigh probability distribution termed the "Beta-Rayleigh" is an improvement over the plain Rayleigh distribution in comparison with measured data. The degree of improvement varies depending on velocity direction and location. The results are summarized in Table 4. The Beta-Rayleigh distribution is a three parameter distribution $\left(U_{m a x}, U_{r m s}, U_{r m q}\right)$ while the Rayleigh distribution is only one parameter. Another improvement is that the Beta-Rayleigh distribution is bounded by a maximum velocity whereas the Rayleigh distribution "tail" trails off into infinity. Also, the Beta-Rayleigh distribution transforms itself into the plain Rayleigh distribution when $U_{m a x}$ approaches infinity and $U_{r m q}=2 \sqrt{2} U_{r m s}^{2}$.

Empirical equations are developed to estimate the parameters of the BetaRayleigh distribution in terms of the following measured parameters of the incident wave spectrum; $T_{p}, H_{m o}$, and water depth, $h$. Good results are achieved with the empirical equations. Correlation coefficients varied between 0.78 and 0.97 . Further research is recommended to broaden the data base from which the empirical equations are developed. Field studies would be particularly helpful. Additional efforts are recommended to investigate the change with elevation $(z)$ of the equations. Eventually, equations could be developed that are a function of $z$, and are applicable throughout the water column. 
Additional recommendations include investigations to determine the variability of velocity statistics with depth, $z$, as well as investigations to determine the thickness of the boundary layer under oscillatory flow. Future studies also could look at theoretical wave reflection from a variable slope and slopes where the reflection coefficient is greater than $20 \%$. 


\section{REFERENCES}

1. Anastasiou, K., Tickell, R.G., and Chaplin, J.R. (1982). "Measurements of Particle Velocities in Laboratory-Scale Random Waves," Coastal Engineering, Vol. 11, 233-254.

2. Battjes, J.A., Van Heteren, J. (1984). "Verification of Linear Theory for Particle Velocities in Wind Waves Based on Field Measurements," Applied Ocean Research, Vol. 6, 187-196.

3. Borgman, L.E. (1986). Unpublished Notes. (Private Collection, N. Sultan)

4. Borgman, L.E. (1982). "Summary of Probability Laws for Wave Properties," Topics in Ocean Physics, Soc. Italiana di Fisica, Bologna, Italy, 373386 .

5. Bouws, E., Gunther, H., Rosenthal, W., and Vincent, C.L. (1985). "Similarity of the Wind Wave Spectrum in Finite Depth Water, Part I Spectral Form." Journal of Geophysical Research, Vol. 90(C1), 975-986.

6. Briggs, M.J. (1991). Private Communication.

7. Bullock, G.N., and Short, I. (1985). "Water Particle Velocities in Regular Waves," Journal of Waterway, Port, Coastal and Ocean Engineering, ASCE, Vol. 111(2), 189-200.

8. CRC Standard Mathematical Tables, 28th Edition (1987). CRC Press Inc., Boca Raton, Florida.

9. Cramér, H. (1946). Mathematical Methods of Statistics. Princeton Universtiy Press, Princeton, New Jersey.

10. Daemrich, K.F., Eggert, W.D., and Cordes, H. (1982). "Investigations on Orbital Velocities and Pressures in Irregular Waves," Proceedings of the 18th Coastal Engineering Conference, Vol. 1, 297-311.

11. Daemrich, K.F., Eggert, W.D., and Kolhase, S. (1980). "Investigations on Irregular Waves in Hydraulic Models," Proceedings of the 17th Coastal Engineering Conference, ASCE, Vol. 1, 186-203.

12. Daily, J.W., and Stephan, S.C., Jr., (1953). "Characteristics of a Solitary Wave," Transactions of the American Society of Civil Engineers, Vol. $118,575-587$.

13. Dean, R.G. (1990). "Water Wave Kinematics: State of the Art and Future Research Needs," In: A. Tørum and O.T. Gudmestad (Editors), Water Wave Kinematics, 743-756. 
14. Dean, R. G., and Dalrymple, R.A. (1984). Water Wave Mechanics for Engineers and Scientists, Prentice-Hall, Inc., Englewood Cliffs, New Jersey.

15. Goda, Y., and Suzuki, Y. (1976). "Estimation of Incident and Reflected Waves in Random Wave Experiments," Proceedings of the 15th Coastal Engineering Conference, ASCE, Vol. 1, 828-845.

16. Goldstein, R.J. (1983) Fluid Mechanics Measurements, Hemisphere Publishing Corp., New York, New York.

17. Hansen, J.B. (1990). "Periodic Waves in the Surf Zone: Analysis of Experimental Data," Coastal Engineering, Vol. 14, 19-41.

18. Honda, T., and Mitsuyasu, H. (1976). "The Statistical Distributions for the Elevation, Velocity, and Acceleration of the Surface of Wind Waves," Rep. Res. Inst. Appl. Mech. (Kyushu Univ.), 24, No. 76, 31-48.

19. Hughes, S.A. (1991) "Estimating Bottom Velocities at a Vertical Wall," To be published March 1992, Journal of Waterway, Port, Coastal and Ocean Engineering, ASCE.

20. Hughes, S.A., and Borgman, L.E. (1987). "Beta-Rayleigh Distribution for Shallow Water Wave Heights," Proceedings of the Conference on Coastal Hydrodynamics, ASCE, 17-31.

21. Hughes, S.A. (1984). "The TMA Shallow-Water Spectrum - Description and Applications." TR-CERC-84-7, U.S. Army Engineer Waterways Station, Coastal Engineering Research Center, Vicksburg, Mississippi.

22. Kim, C.H., Randall, R.E., Krafft, M.J., and Boo, S.Y. (1990). "Experimental Study of Kinematics of Large Transient Wave in 2-D Wave Tank," Proceedings of the 22nd Annual Offshore Technology Conference, Houston, 195-202.

23. Laser Doppler Anemometry (1983). Dantec Electronics, Inc., Allendale, New Jersey.

24. Lee, J.J., Skjelbreia, A.M., and Raichlen, F. (1982). "Measurement of Velocities in Solitary Waves," Journal of the Waterway, Port, Coastal and Ocean Division, ASCE, Vol. 108 (No. WW2), 200-218.

25. Longuet-Higgins, M.S. (1963). "The Effect of Non-Linearities on Statistical Distributions in the Theory of Sea Waves," Journal of Fluid Mechanics, 17, Part 3, 459-480.

26. Longuet-Higgins, M.S. (1952). "On the Statistical Distribution of the Heights of Sea Waves," Journal of Marine Research, Vol. 11, No. 3, 245266. 
27. McCowan J. (1894). "On the Highest Wave of Permanent Type," Philos. Mag. J. Sci., Vol. 38.

28. Munson, B.R., Young, D.F., and Okiishi, T.H. (1990). Fundamentals of Fluid Mechanics, John Wiley \& Sons, Inc., New York, New York, 487-497.

29. Nielsen, P. (1982). "Explicit Formulae for Practical Wave Calculations," Coastal Engineering, Vol. 6, 389-398.

30. Ochi, M.K. (1982). In: V.T. Chow (Editor), Advances in Hydroscience, Academic Press, New York, New York, 348-365.

31. Rice, O.S. (1944). "Mathematical Analysis of Random Noise," Bell Systems Technical Journal, 23, 282-332.

32. Shore Protection Manual(1984). Coastal Engineering Research Center, U.S. Army Corps of Engineers, Washington, D.C..

33. Soulsby, R.L. (1987). "Calculating Bottom Orbital Velocity Beneath Waves," Coastal Engineering, Vol. 11, 371-380.

34. St. Denis, M. (1975). "On Statistical Techniques for Predicting the Extreme Dimensions of Ocean Waves and of Amplitudes of Ship Responses," Proc. Ship Technol. Res. Symp., Soc. Nav. Archit. Mar. Eng., 1st, 1975 pp. 3.1-3.26.

35. Swan, C. (1990). "Convection within an Experimental Wave Flume," Journal of Hydraulic Research, Vol. 28, 273-282.

36. Tayfun, M.A. (1980). "Narrow-band Nonlinear Sea Waves," J. Geophys. Res., 85, No. C3, 1548-1552.

37. Vis, F.C. (1980) "Orbital Velocities in Irregular Waves," Proceedings of the 17th Coastal Engineering Conference, ASCE, Vol. 1, 173-185.

38. Zhang, J., Randall, R.E., and Spell, C.A. (1991). "On Wave Kinematics Approximate Methods," Proceedings 23rd Annual Offshore Technology Conference, Houston, 231-238. 


\section{APPENDIX I: SUPPLEMENTAL GRAPHS}




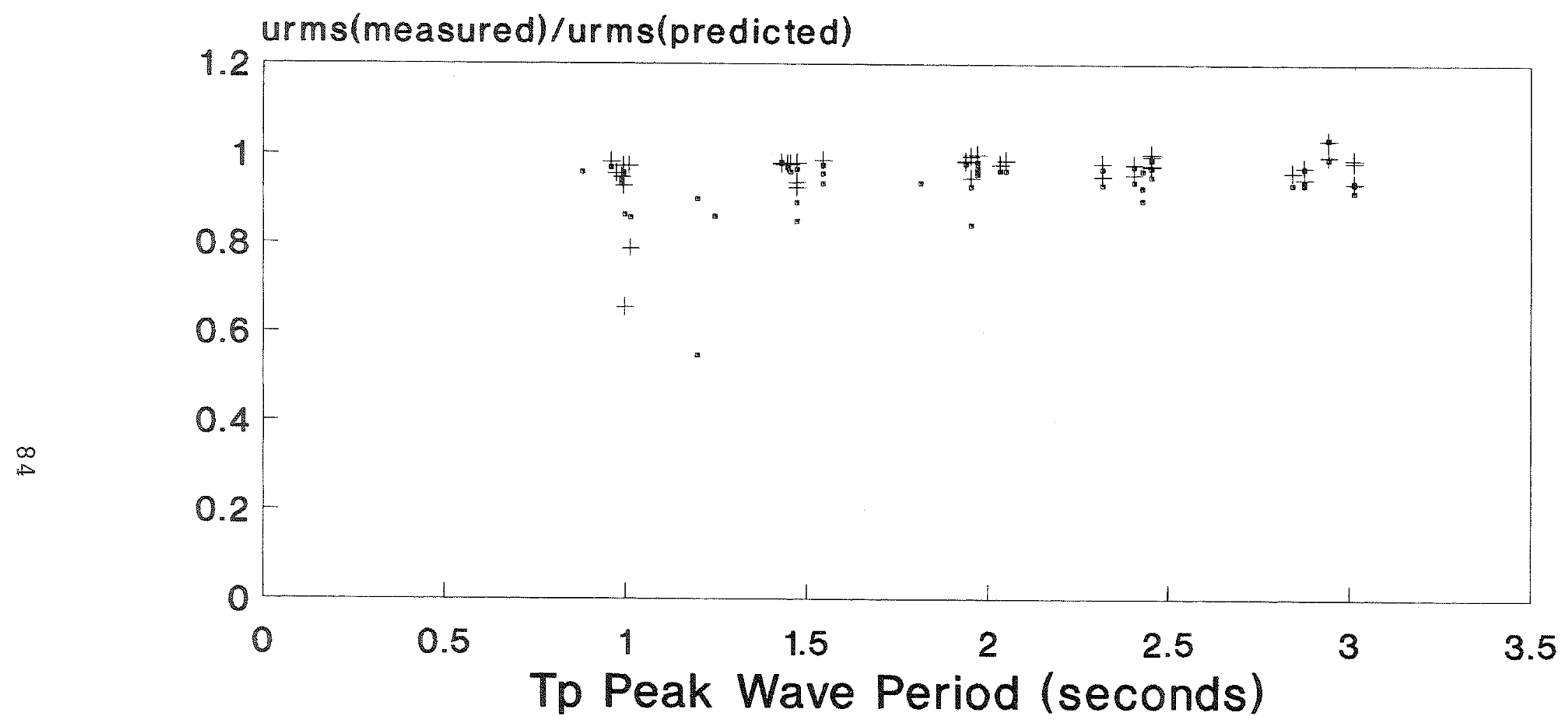

- $z=$ mid-depth $\quad+\quad z=$ near bottom

Figure 37: $u_{r m s}$ Measured/Predicted vs. Peak Spectral Period 
$\stackrel{\infty}{\sim}$

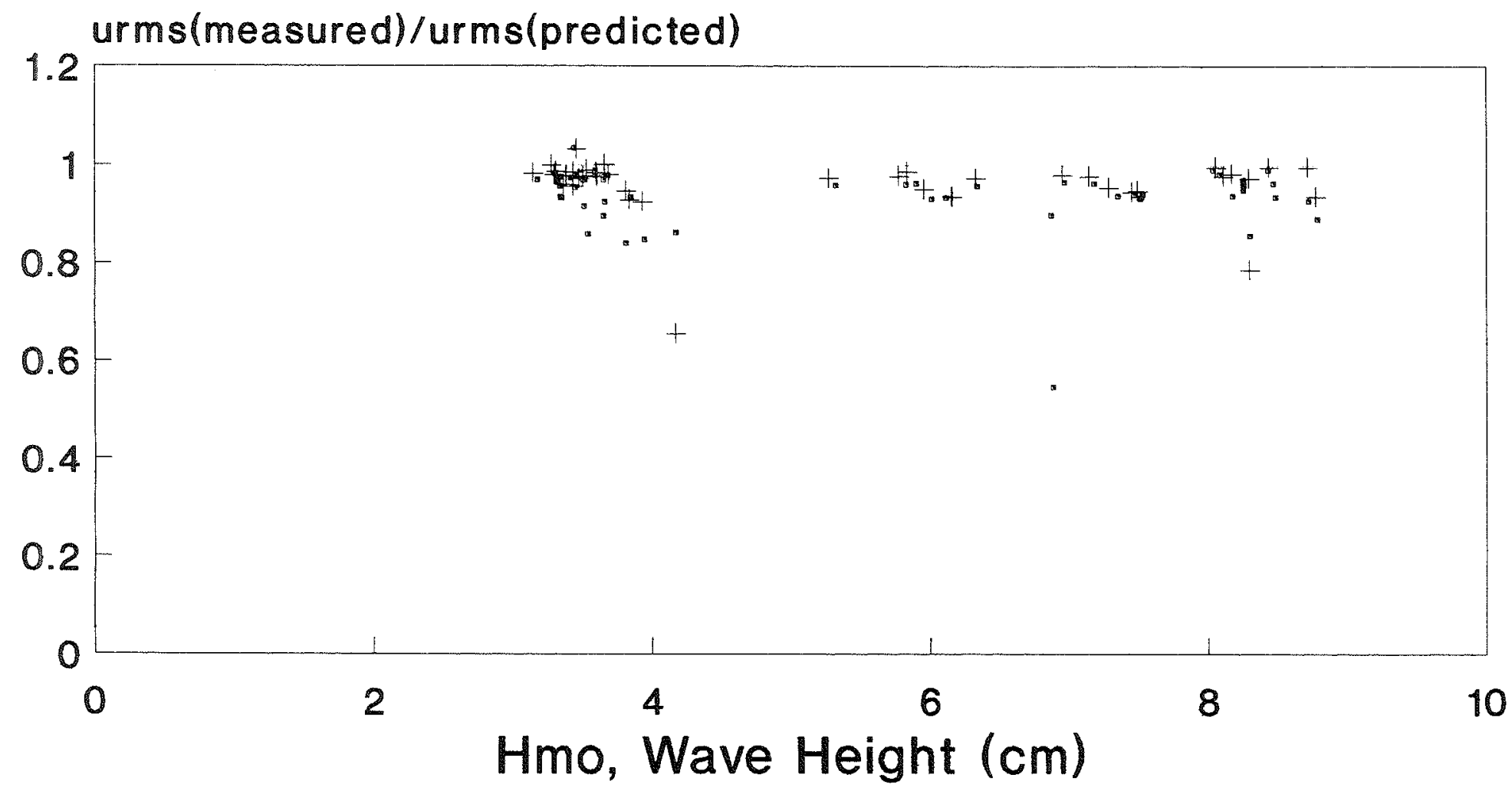

$z=$ mid-depth $\quad+\quad z=$ near bottom

Figure 38: $u_{r m s}$ Measured/Predicted vs. Spectral Significant Wave Height 


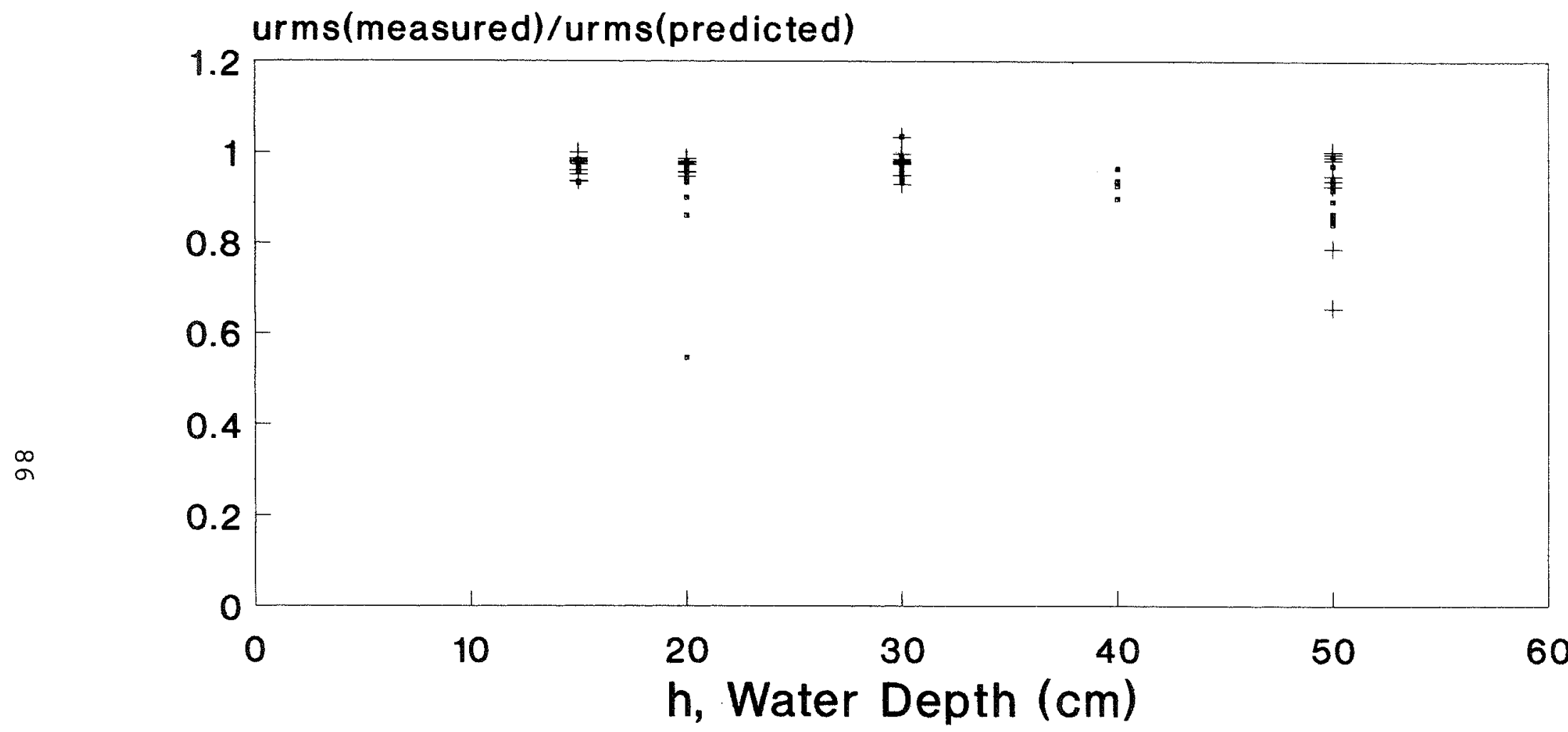

- $\mathbf{z}=$ mid-depth $\quad+\quad z=$ near bottom

Figure 39: $u_{r m s}$ Measured/Predicted vs. Water Depth 


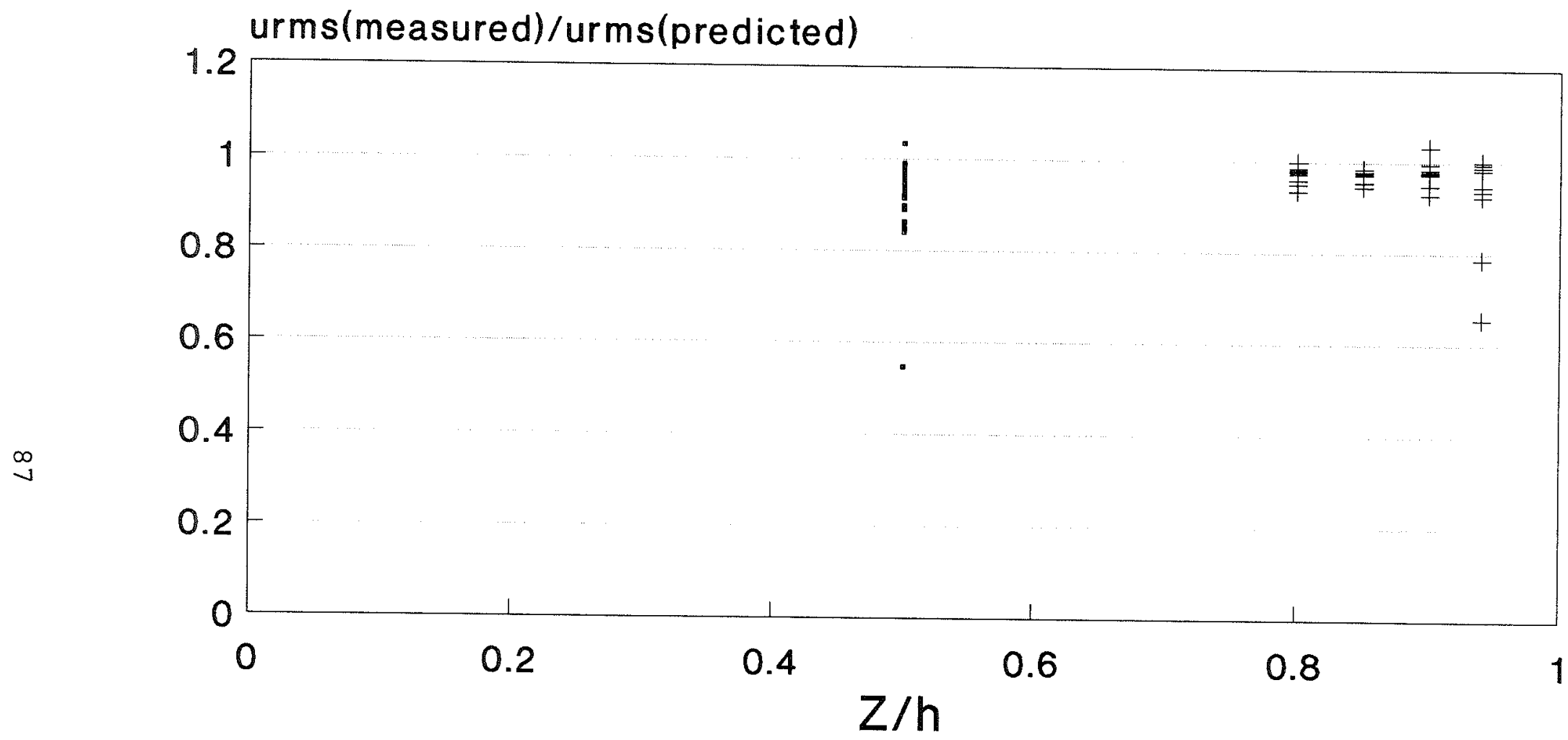

- $z=$ mid-depth $\quad+\quad z=$ near bottom

Figure 40: $u_{r m s}$ Measured/Predicted vs. $Z / h$ 


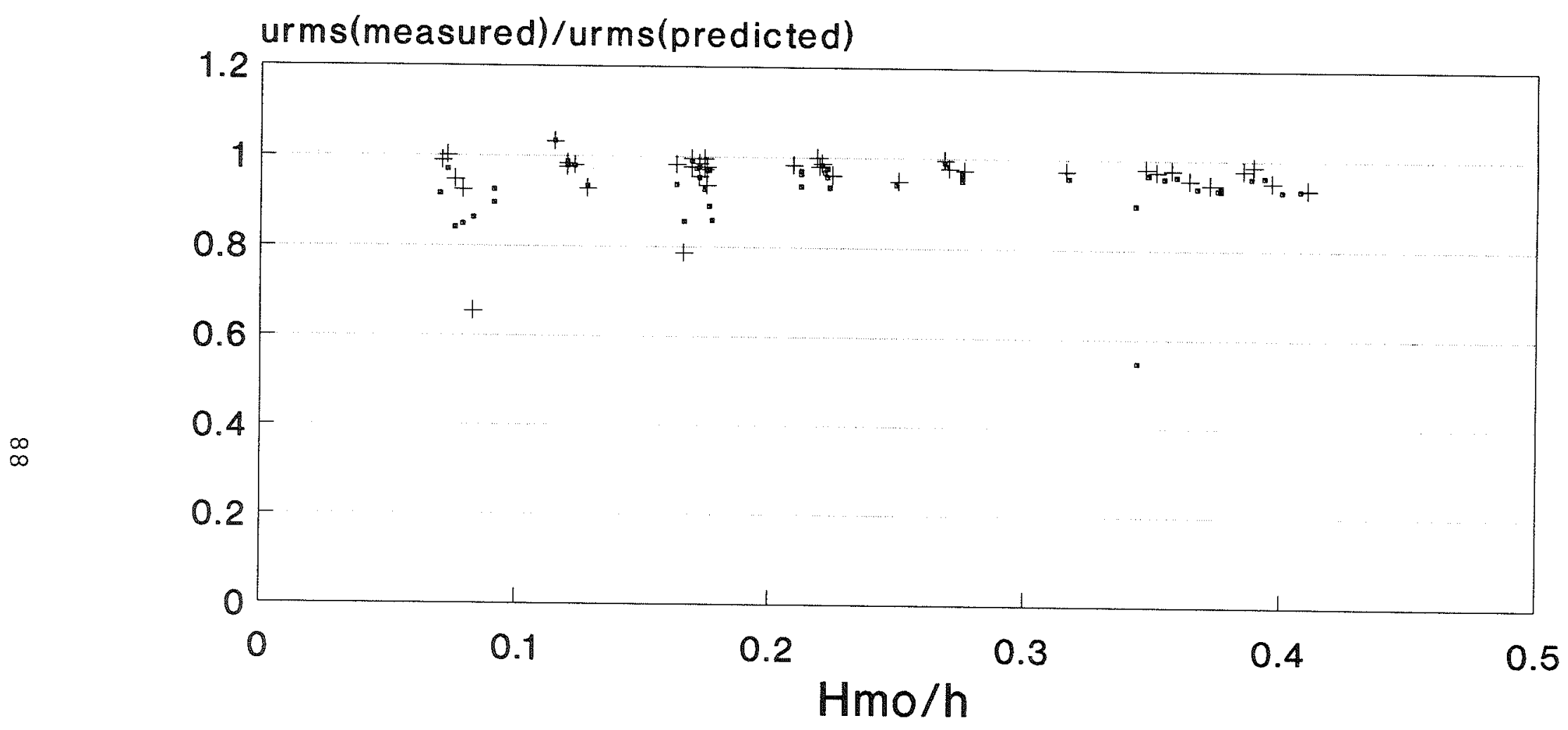

- $z=$ mid-depth $\quad+\quad z=$ near bottom

Figure 41: $u_{r m s}$ Measured/Predicted vs. $H_{m o} / h$ 


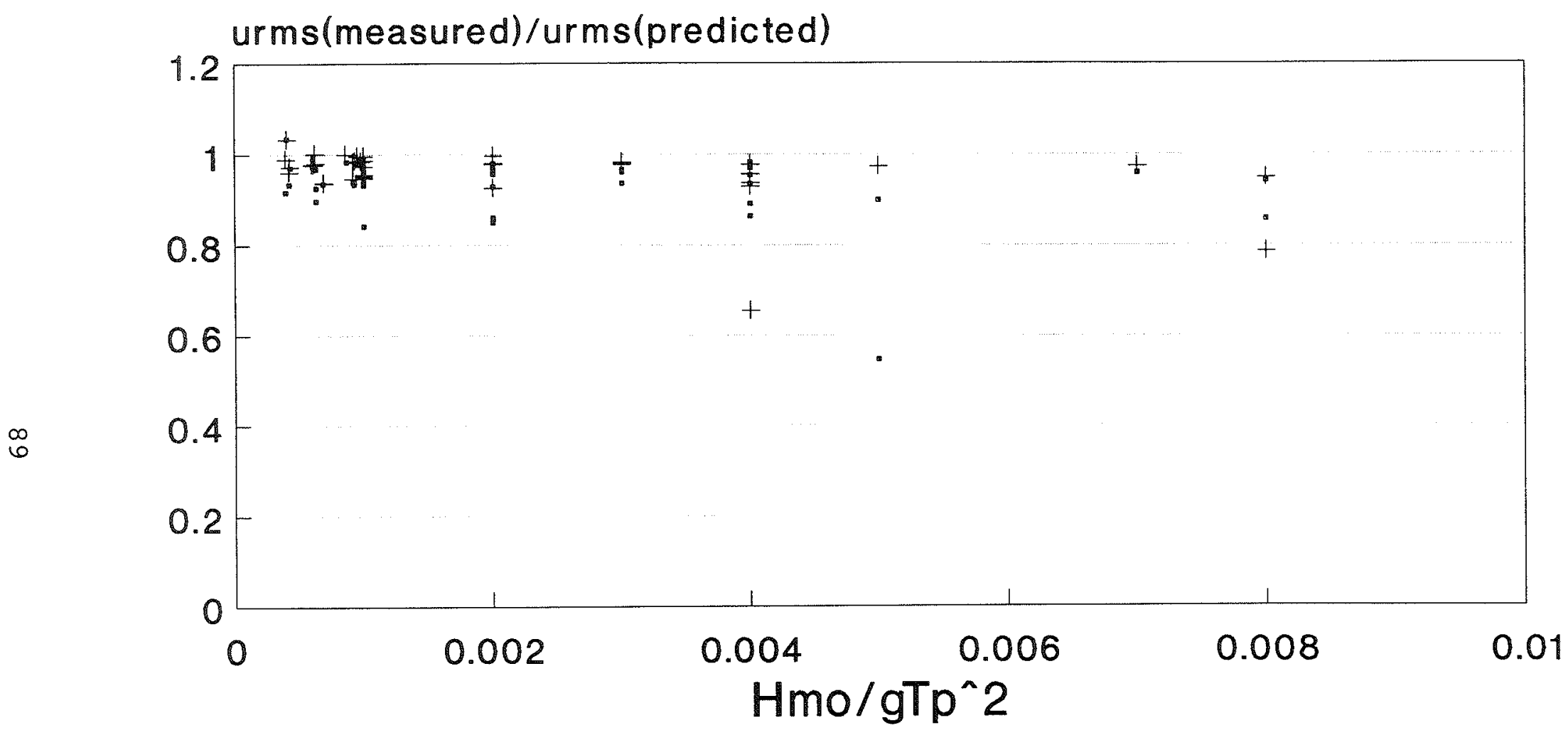

- $z=$ mid-depth $\quad+\quad z=$ near bottom

Figure 42: $u_{r m s}$ Measured/Predicted vs. $H_{m o} / g T_{p}^{2}$ 


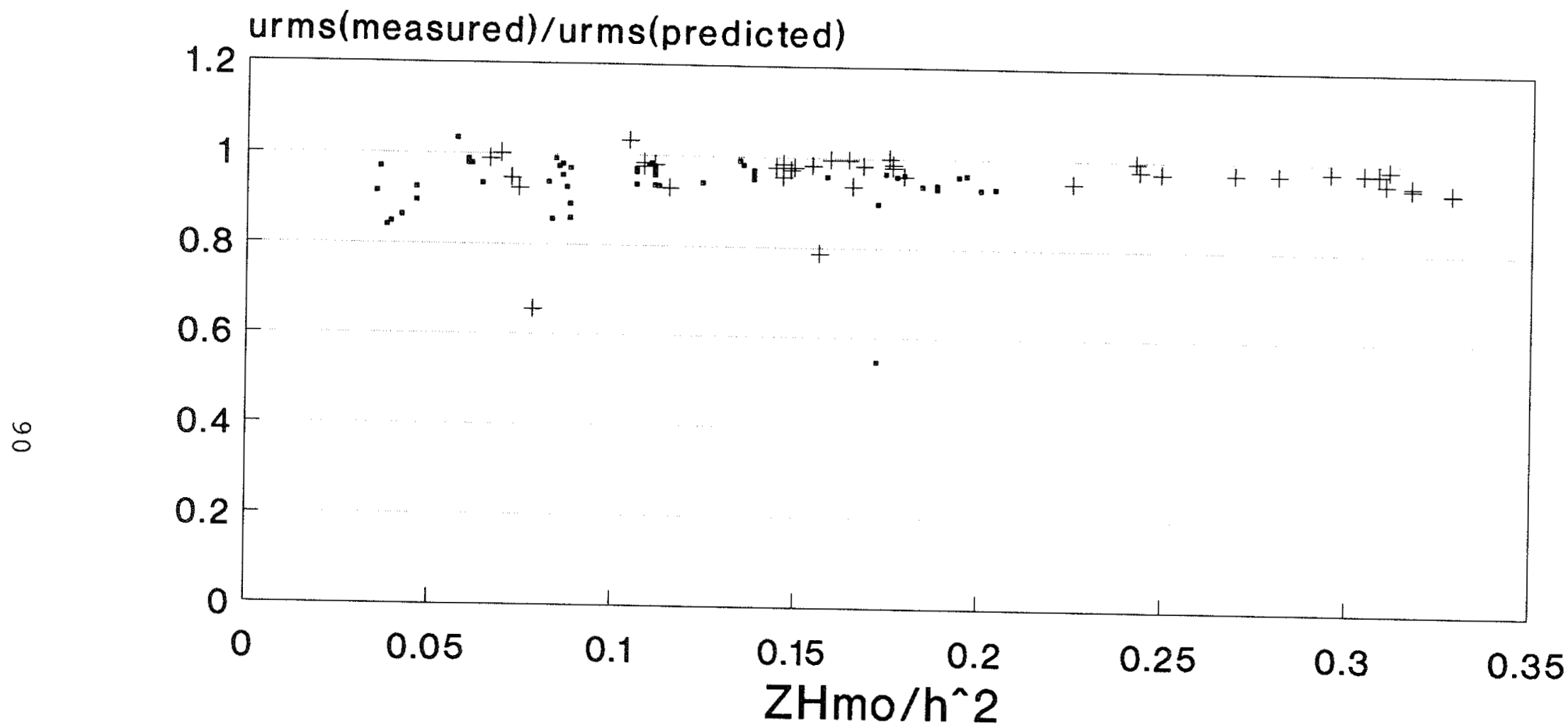

- $z$ =mid-depth $\quad+\quad z=$ near bottom

Figure 43: $u_{r m s}$ Measured/Predicted vs. $Z H_{m o} / h^{2}$ 


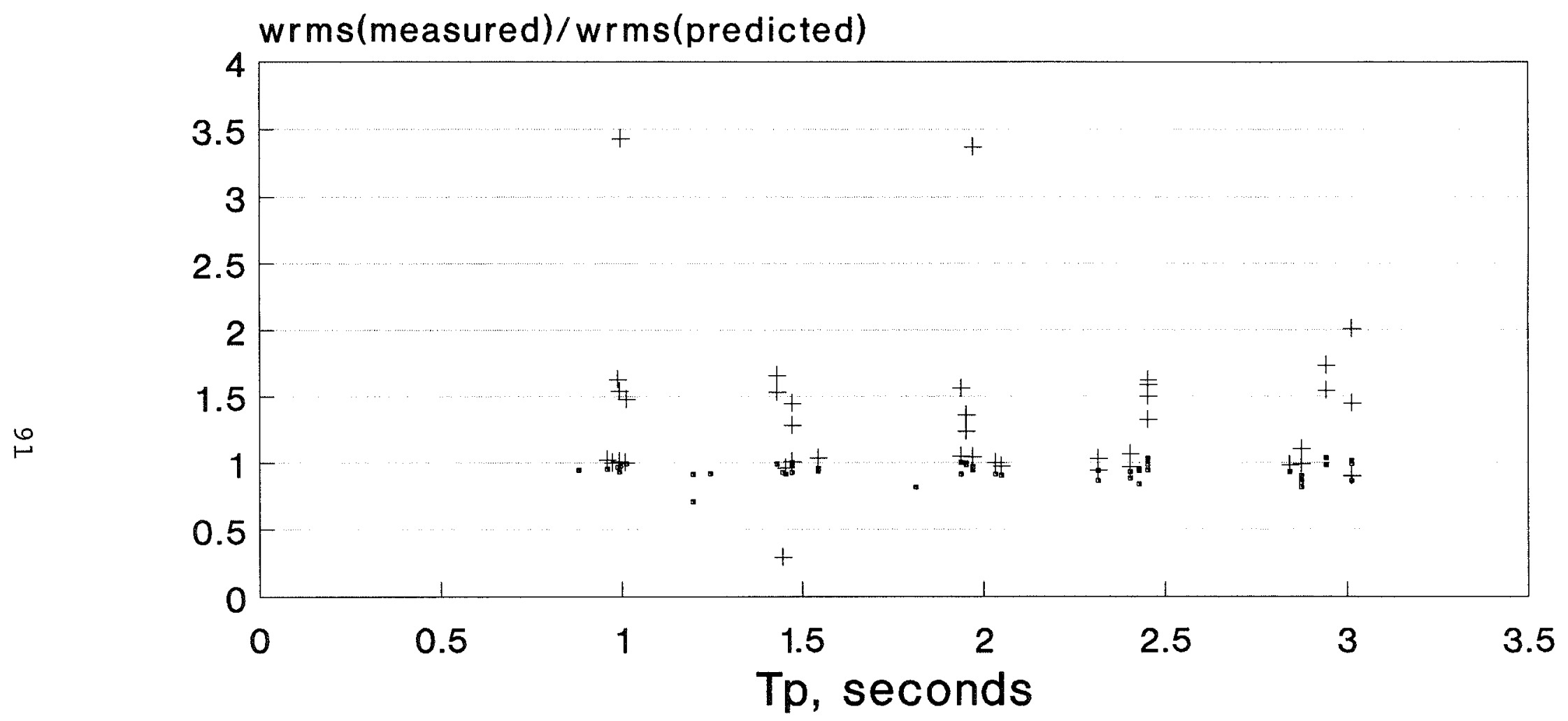

- $z=$ mid-depth $\quad+\quad z=$ near bottom

Figure 44: $w_{r m s}$ Measured/Predicted vs. Peak Spectral Period 


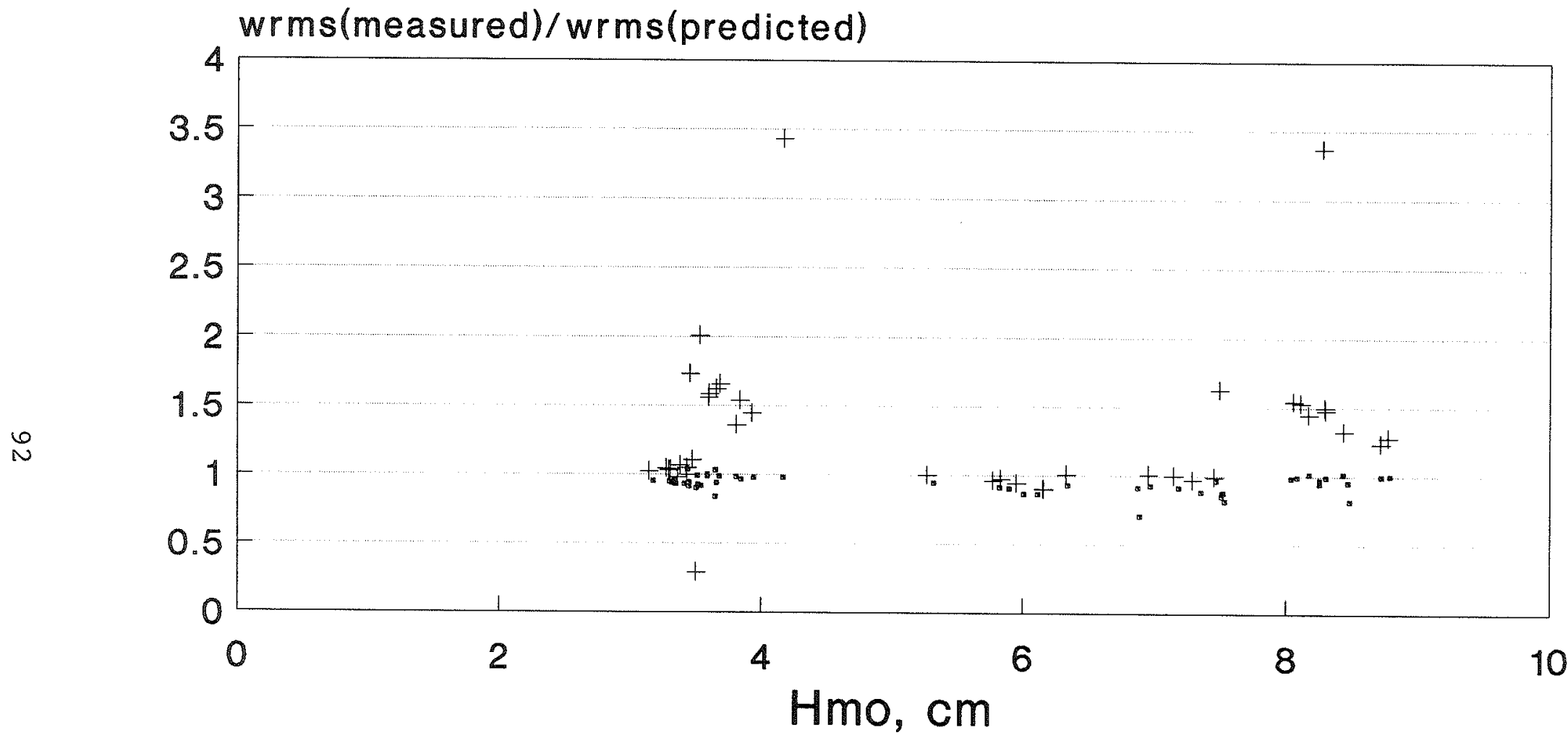

- $z=$ mid-depth $\quad+\quad z=$ near bottom

Figure 45: $w_{r m s}$ Measured/Predicted vs. Spectral Significant Wave Height 


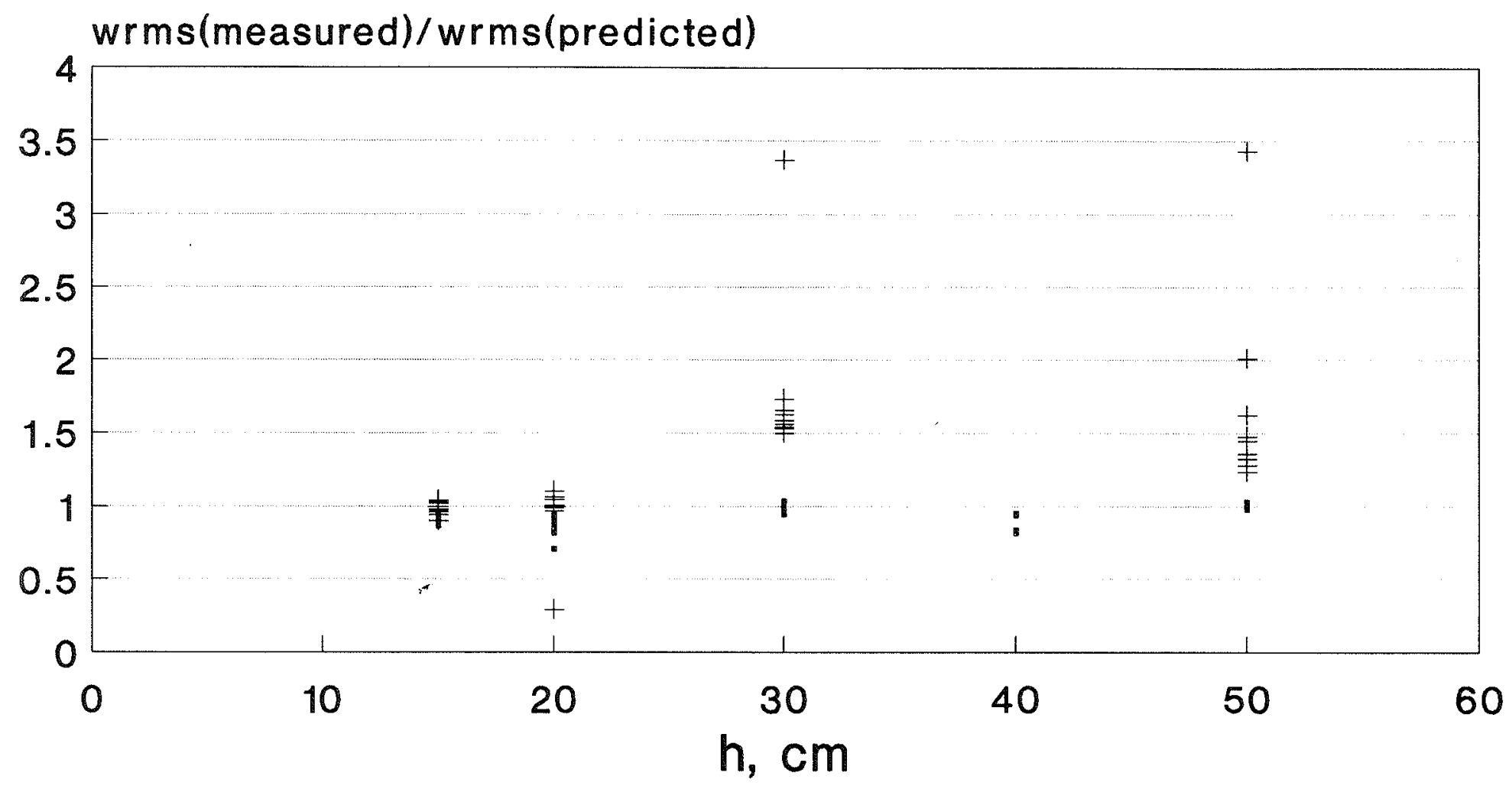

- $z$ =mid-depth $+z=$ near bottom

Figure 46: $w_{\text {rms }}$ Measured/Predicted vs. Water Depth 


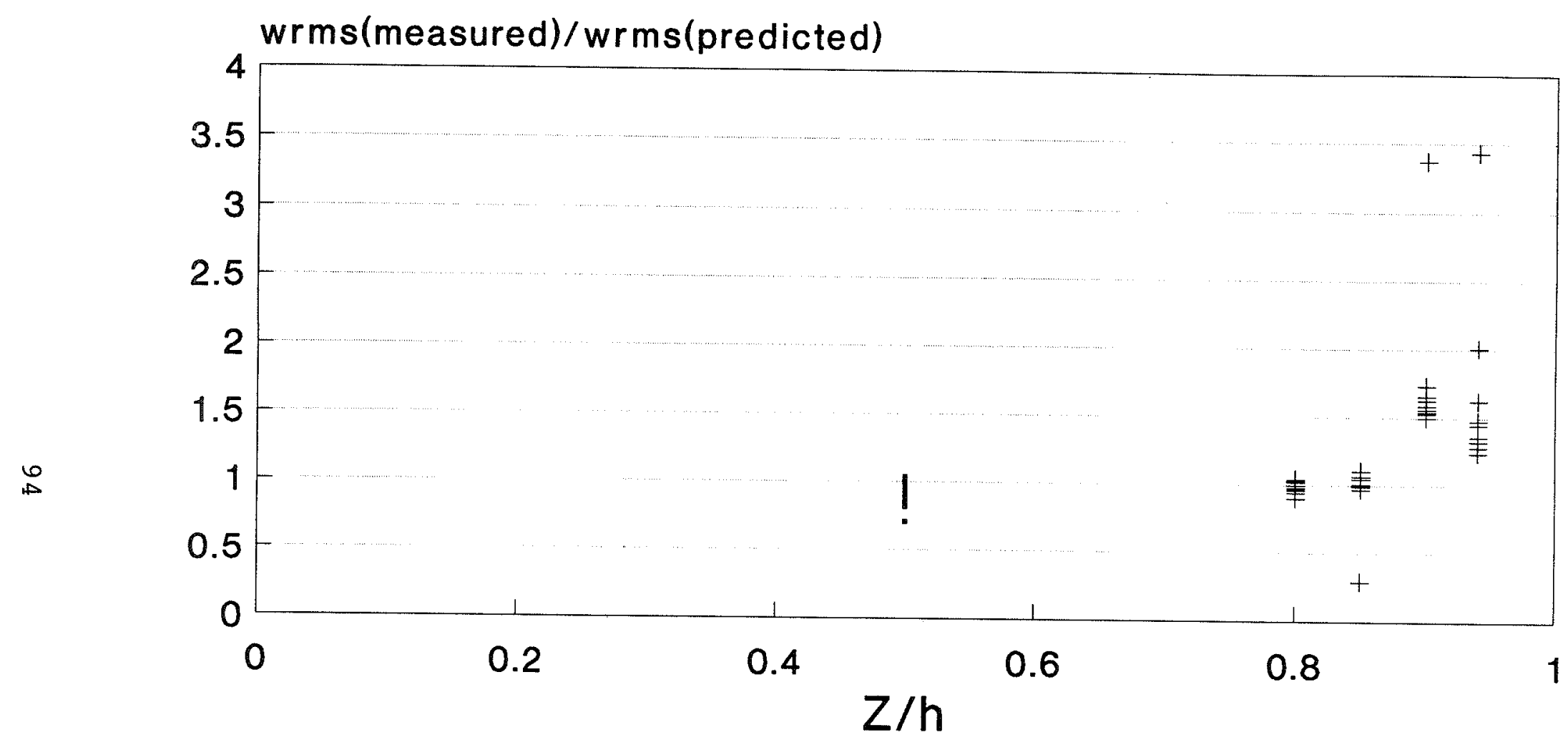

- $z$ =mid-depth $\quad+\quad z=$ near bottom

Figure 47: $w_{r m s}$ Measured/Predicted vs. $Z / h$ 


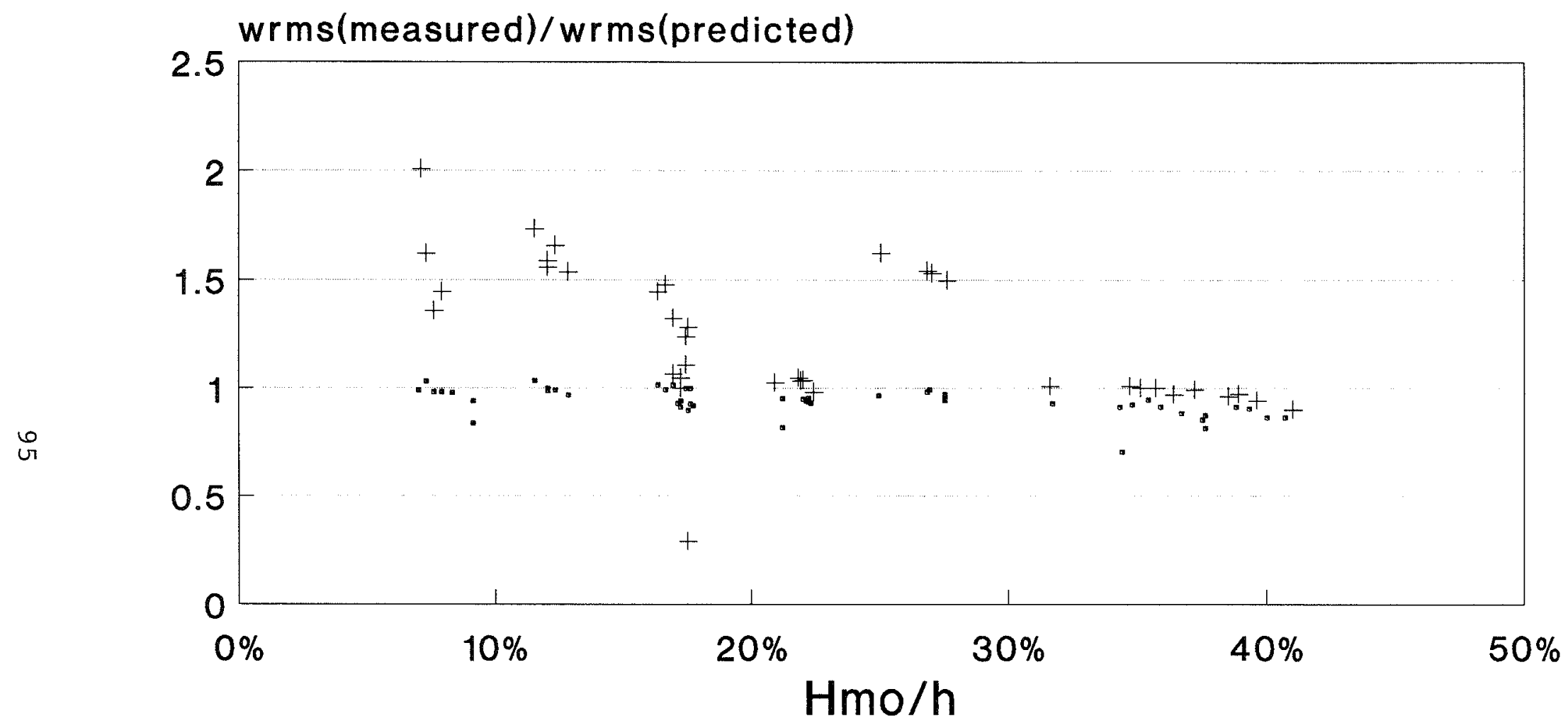

- $z=$ mid-depth $+z$ =near bottom

Figure 48: $w_{r m s}$ Measured/Predicted vs. $H_{m o} / h$ 


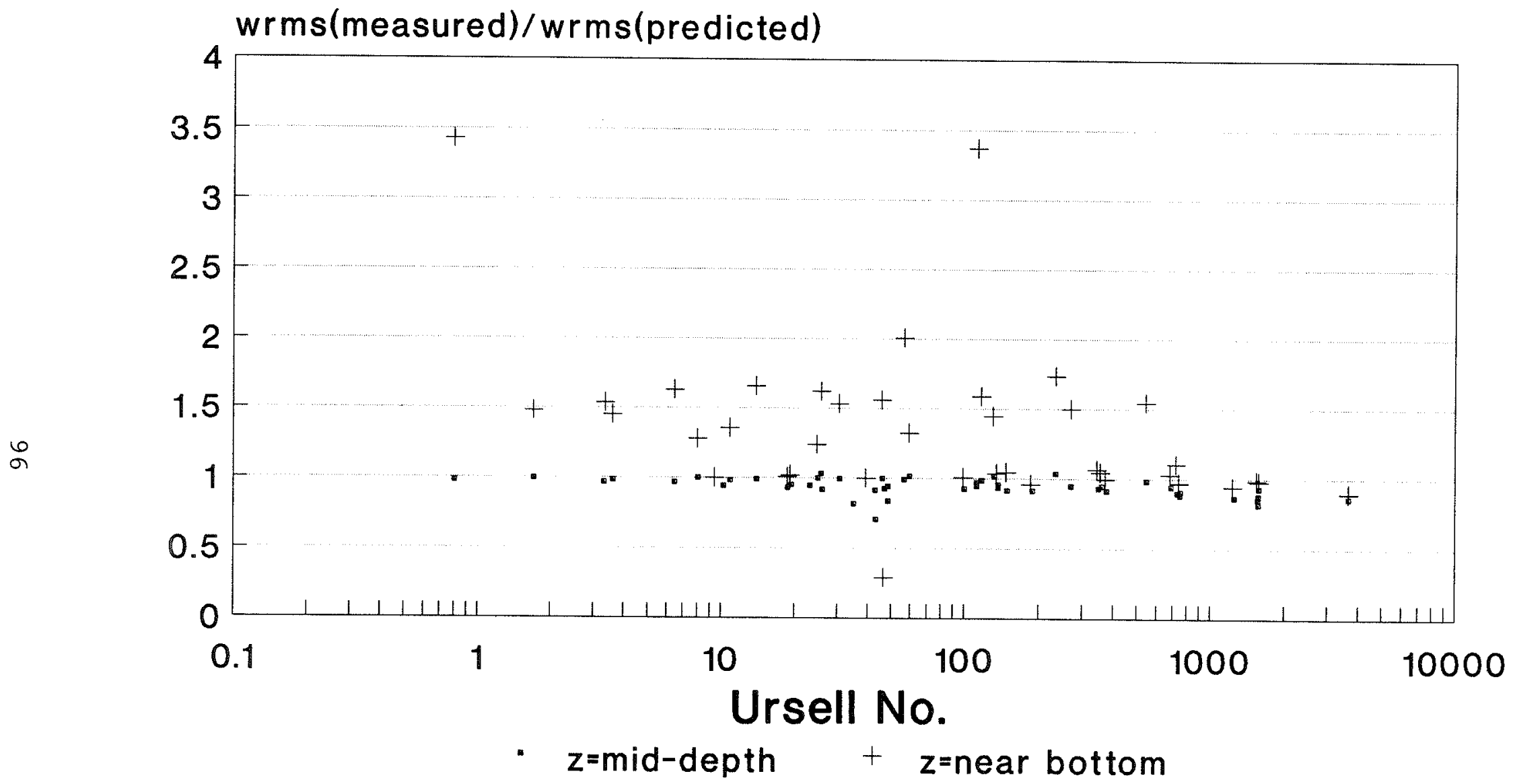

Figure 49: $w_{r m s}$ Measured/Predicted vs. Ursell No. (without outliers) 


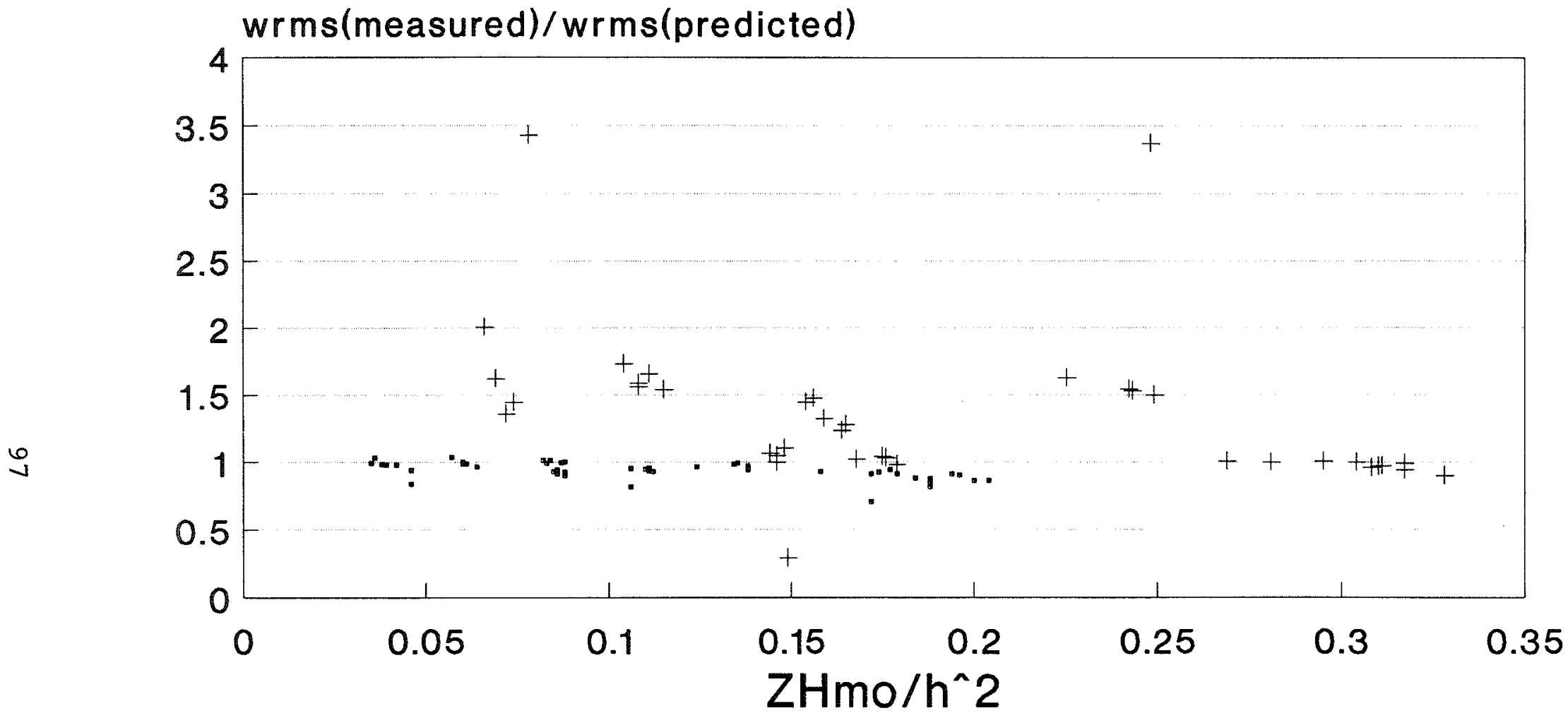

- $z$ =mid-depth $+z$ =near bottom

Figure 50: $w_{r m s}$ Measured/Predicted vs. $Z H_{m o} / h^{2}$ 


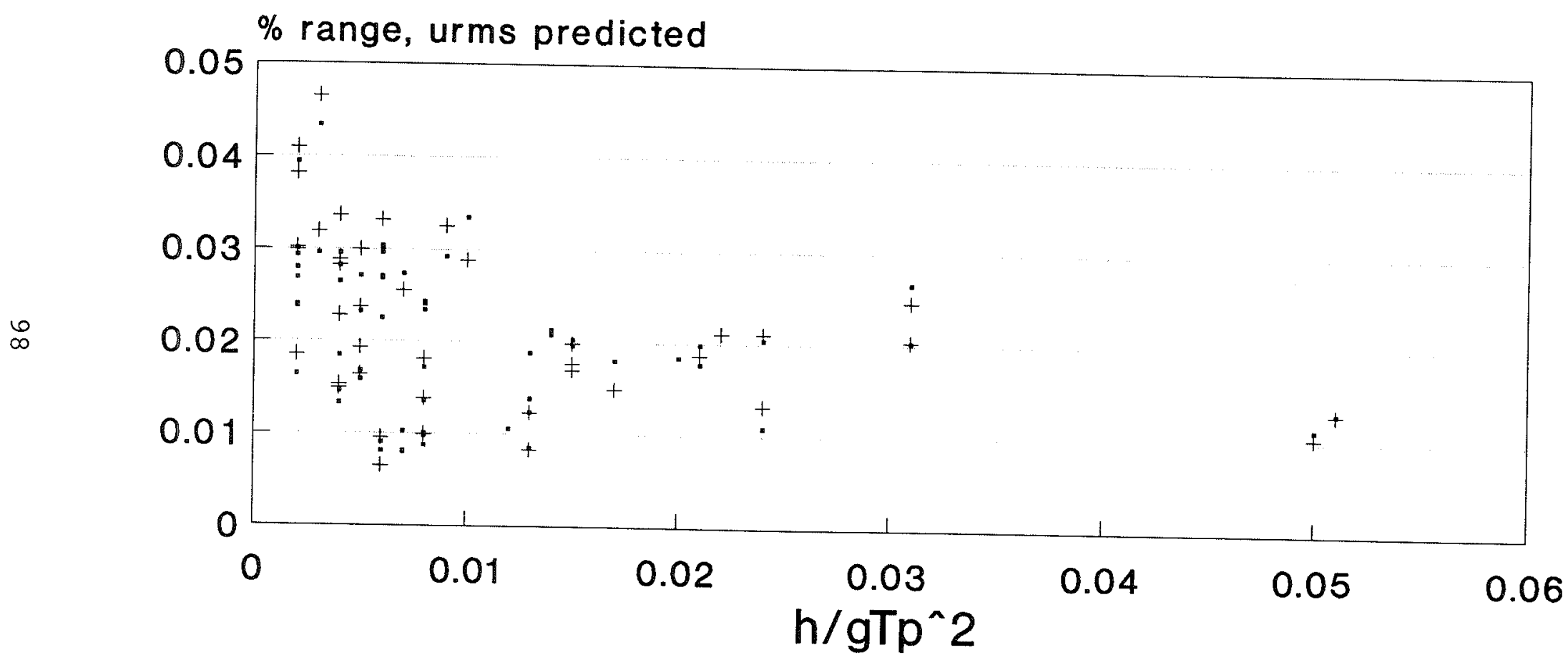

- $U, z=$ mid-depth $\quad+\quad U, z=$ near bottom

Figure 51: \%-Range of $u_{r m s}$ Predicted as a function of X, (Measured Phase Angle) 


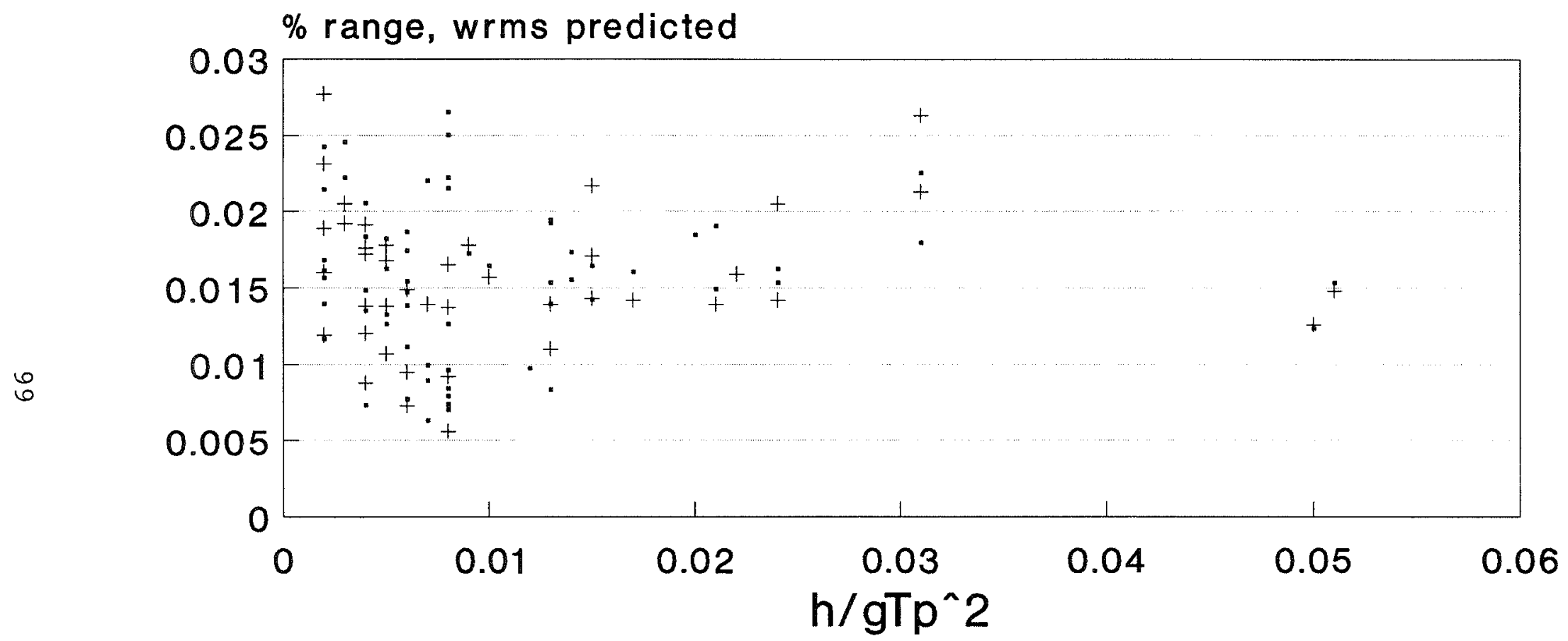

- W, z=mid-depth $\quad+\quad W, z=$ near bottom

Figure 52: \%-Range of $w_{r m s}$ Predicted as a function of X, (Random Phase Angle) 


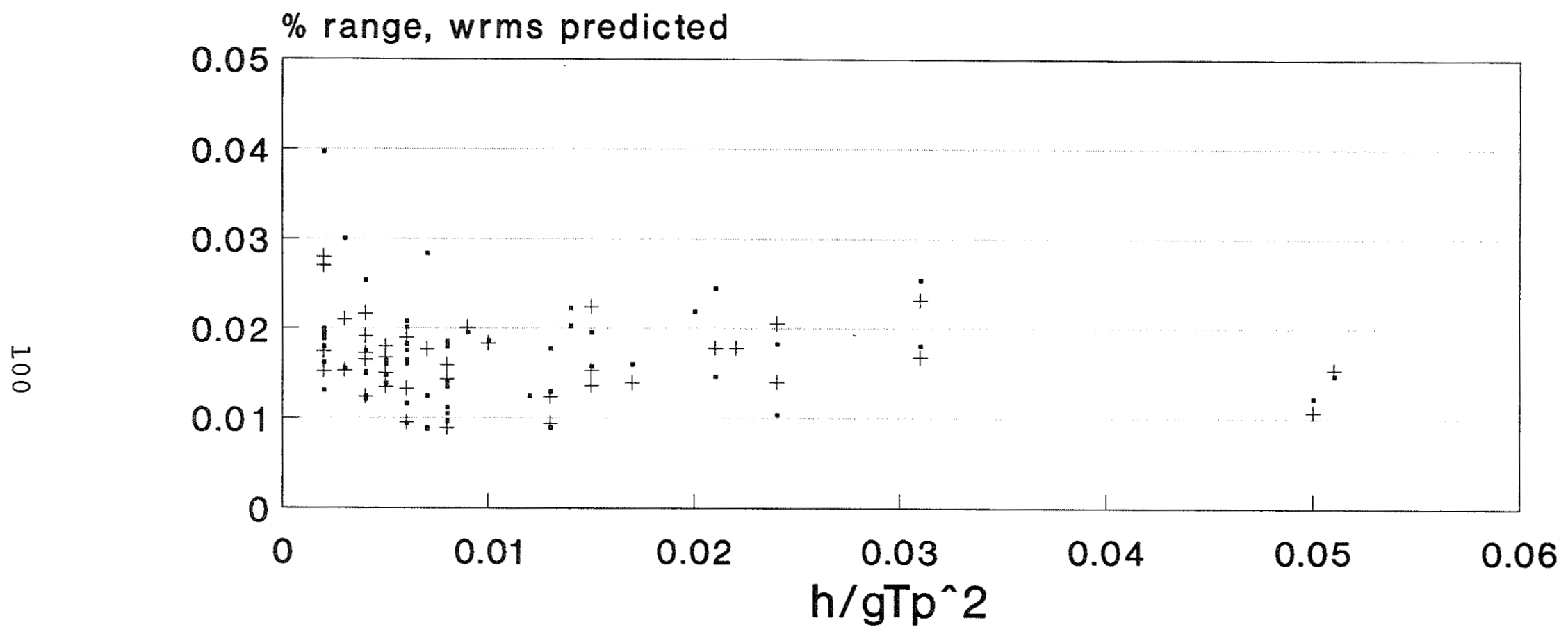

- $W, z=$ mid-depth $+W, z=$ near bottom

Figure 53: \%-Range of $w_{r m s}$ Predicted as a function of X, (Measured Phase Angle) 
No. Waves(waves)/No. Waves(velocities)

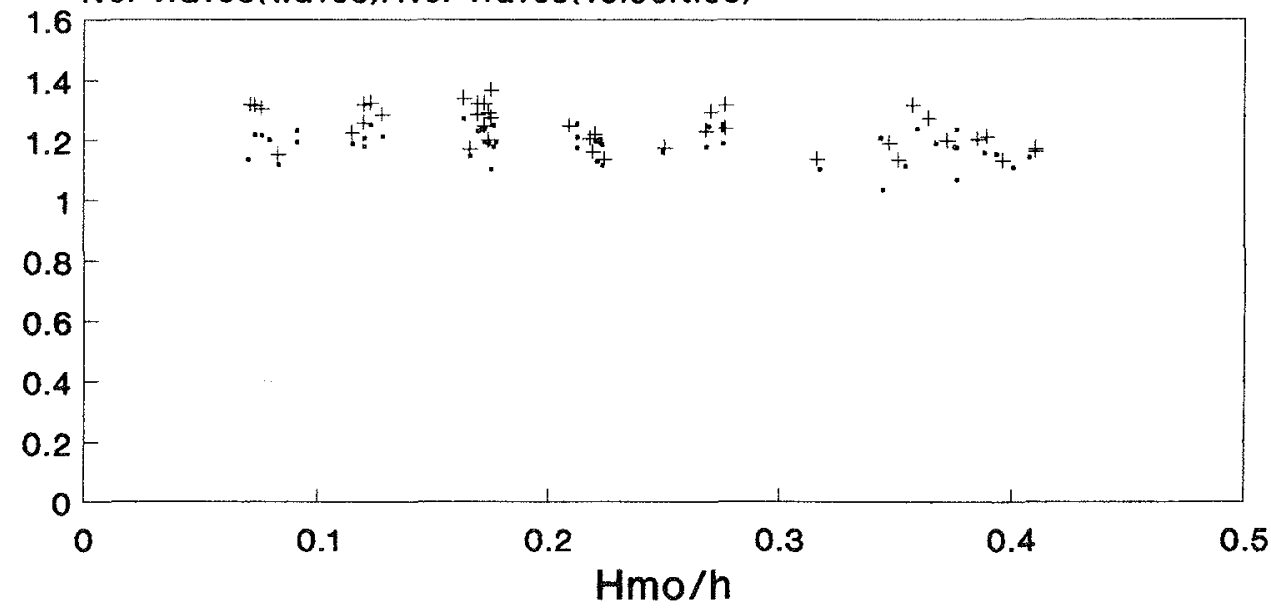

- U, z=mid-depth $+U, z=$ near bottom

(a)

No. Waves(waves)/No. Waves(velocities)

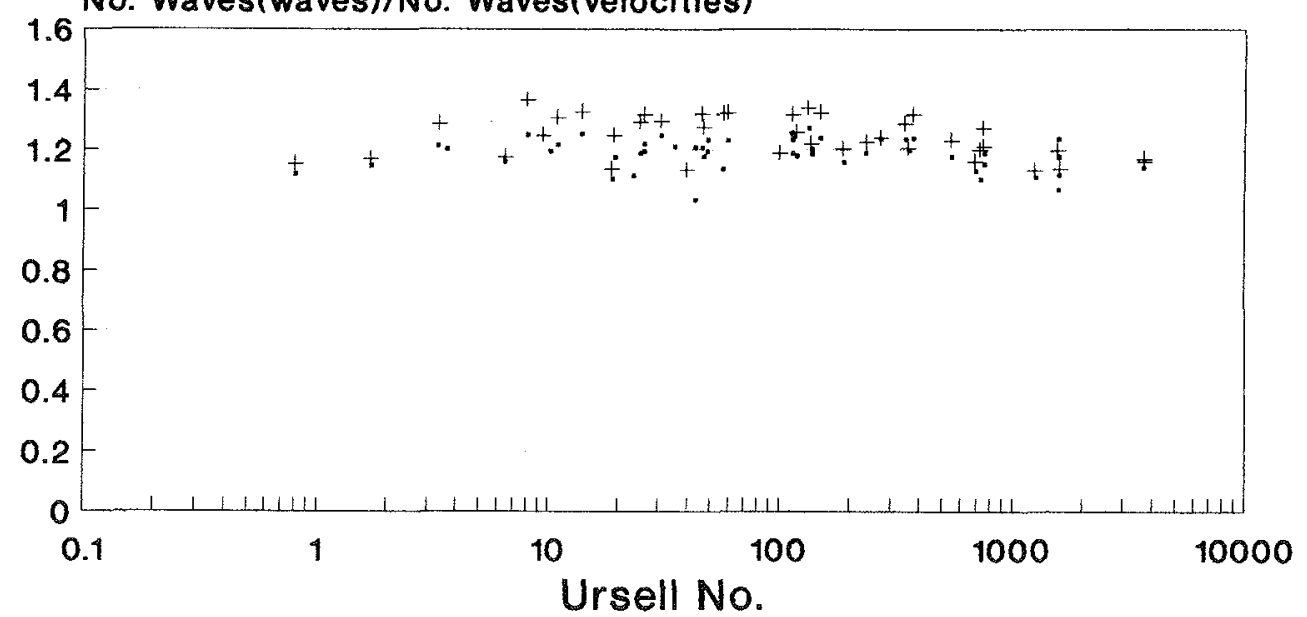

(b)

$U, z$ mid-depth $+U, z$ near bottom

Figure 54: No. Waves Counted, Horizontal Direction. (a) vs, $H_{m o} / h$; (b) vs. Ursell No. 


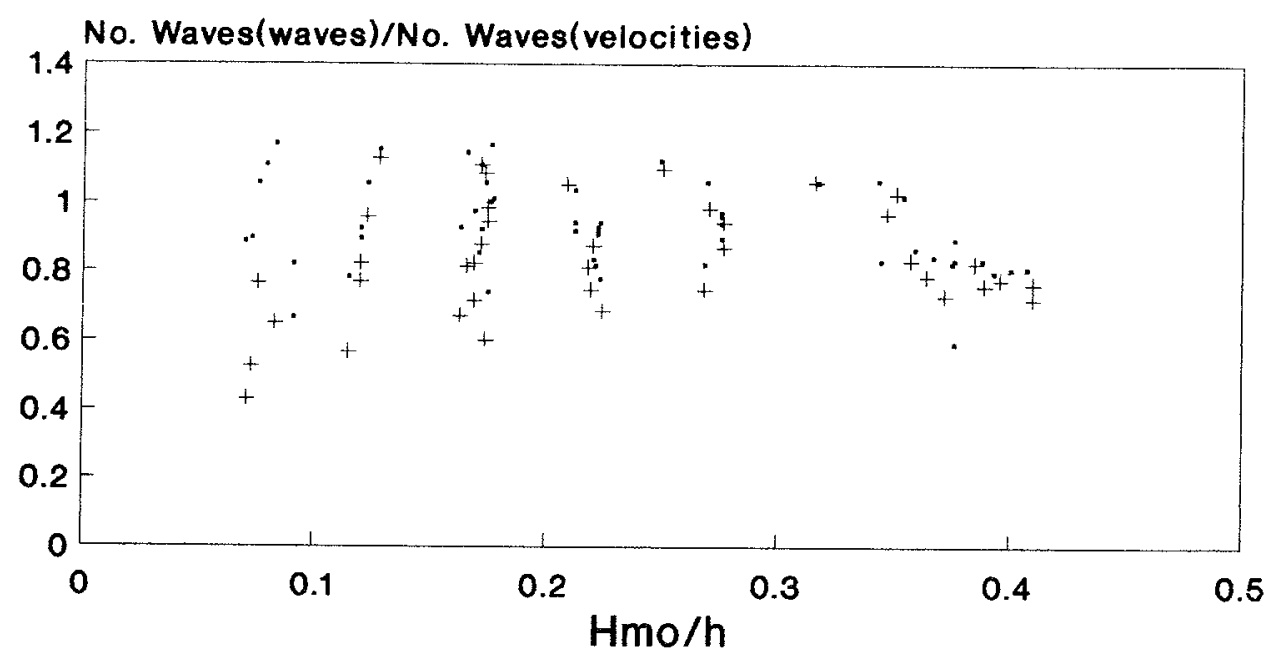

- $\mathbf{W}, \mathbf{z}=$ mid-depth $\quad+\quad W, z=n e a r$ bottom

(a)

No. Waves(waves)/No. Waves(velocities)

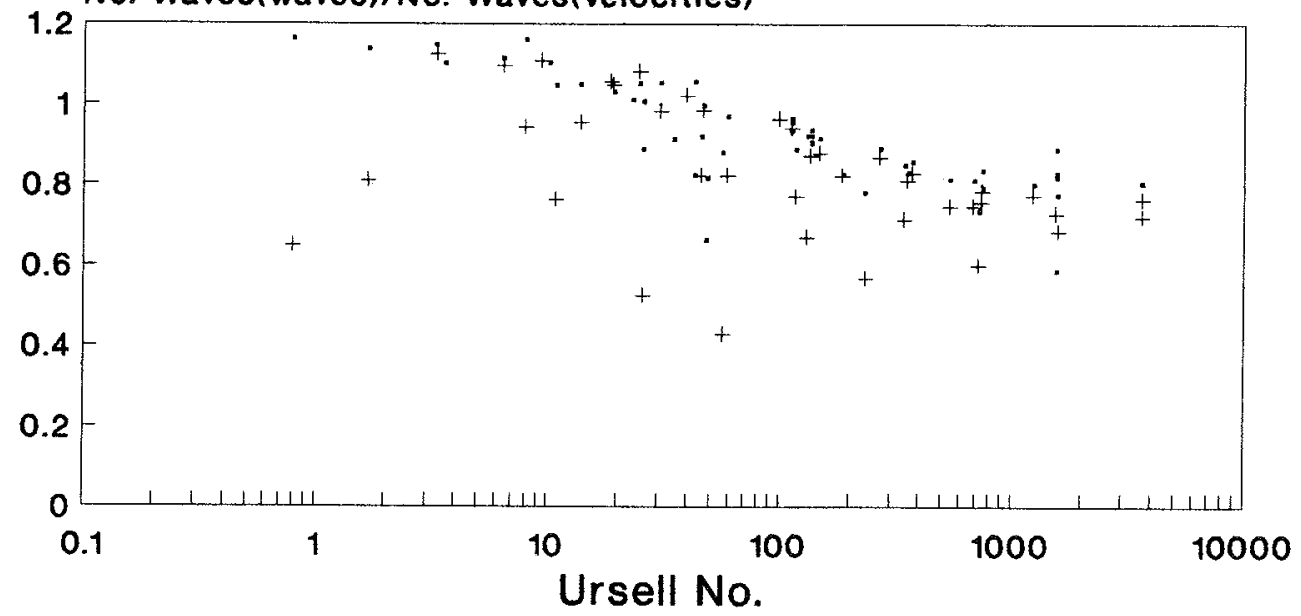

W, z-mid-depth + W, z-near bottom

(b)

Figure 55: No. Waves Counted, Vertical Direction. (a) vs. $H_{m o} / h$; (b) vs. Ursell No. 


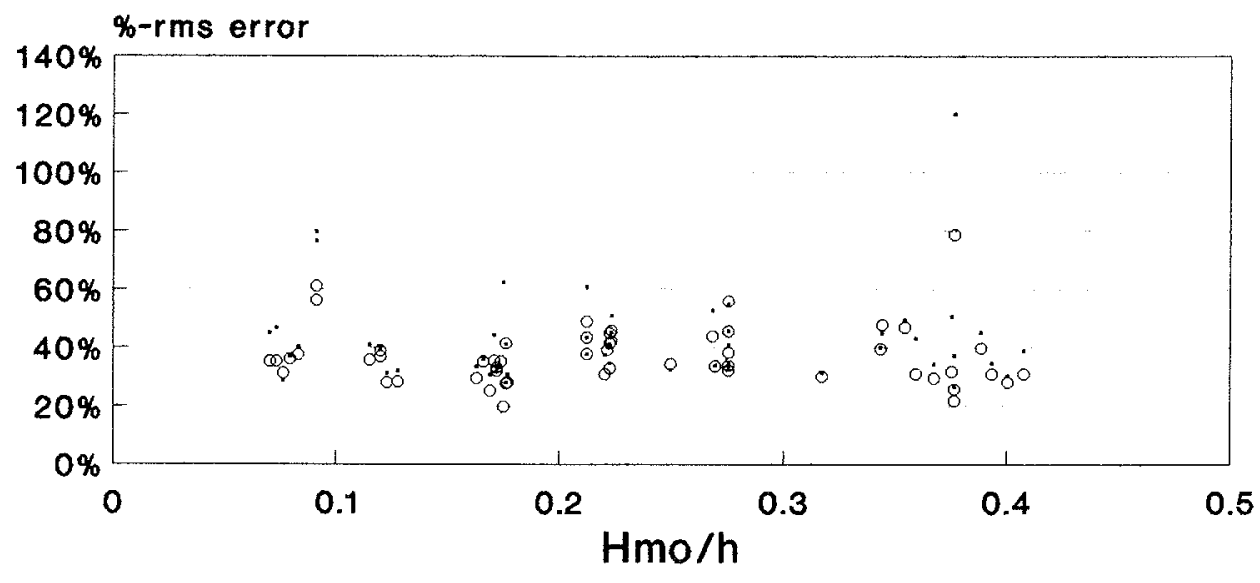

Rayleigh,z=mid-depth $\quad \circ$ Beta-R, z-mid-depth

(a)

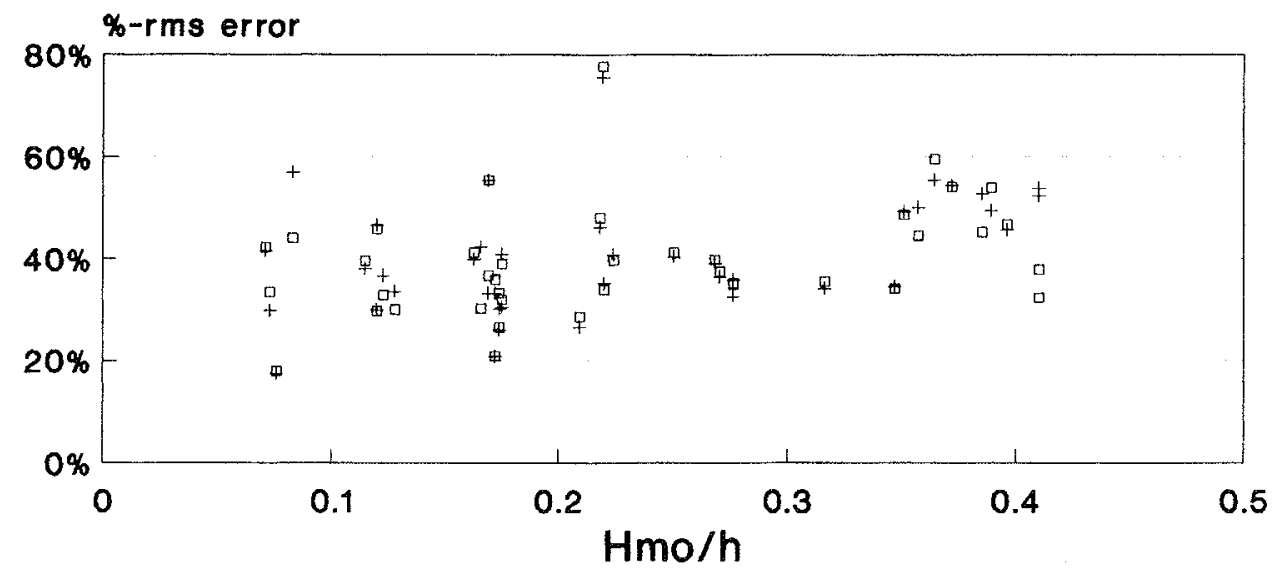

+ Rayleigh,z=bottom $\square$ Beta-R, z=bottom

(b)

Figure 56: \%-Root-mean-square Error, Beta-Rayleigh Distribution, Positive Horizontal Direction. (a) Mid-depth; (b) Near Bottom 


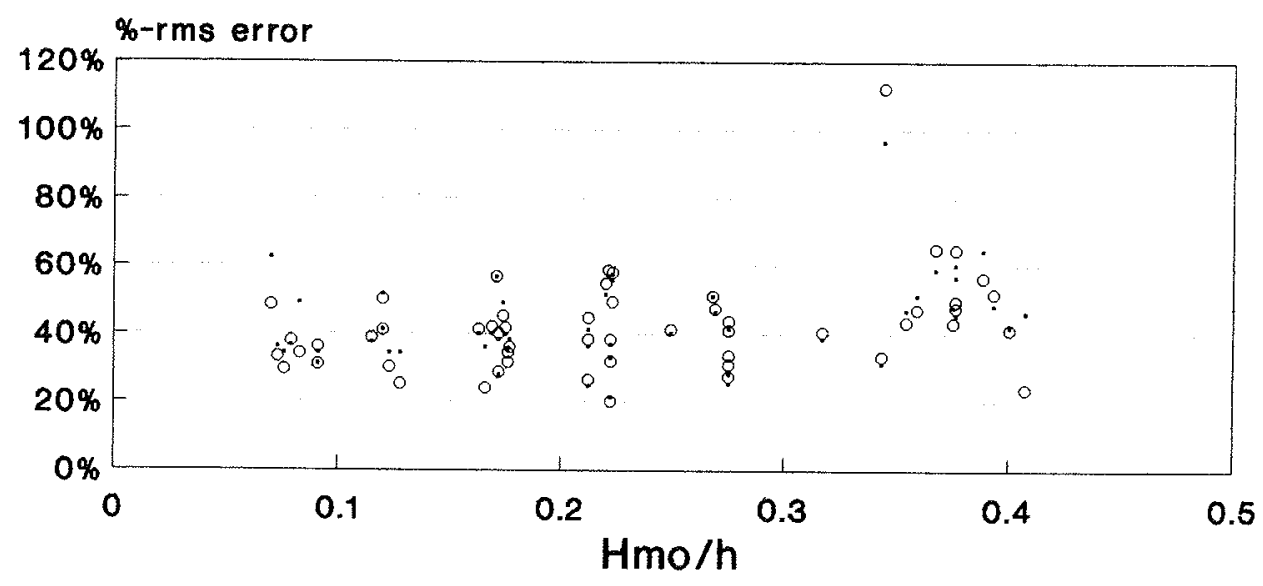

Rayleigh,z=mid-depth $\quad \circ$ Beta-R, z-mid-depth

(a)

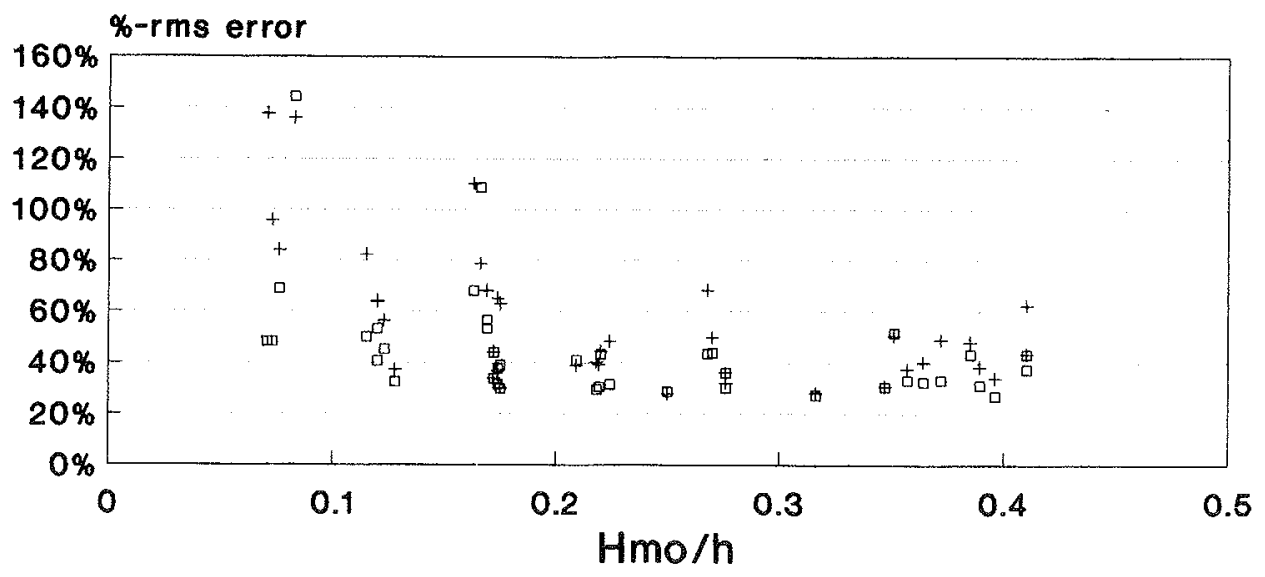

+ Rayleigh,z=bottom $\quad$ Beta-R, zabottom

(b)

Figure 57: \%-Root-mean-square Error, Beta-Rayleigh Distribution, Positive Vertical Direction. (a) Mid-depth; (b) Near Bottom 


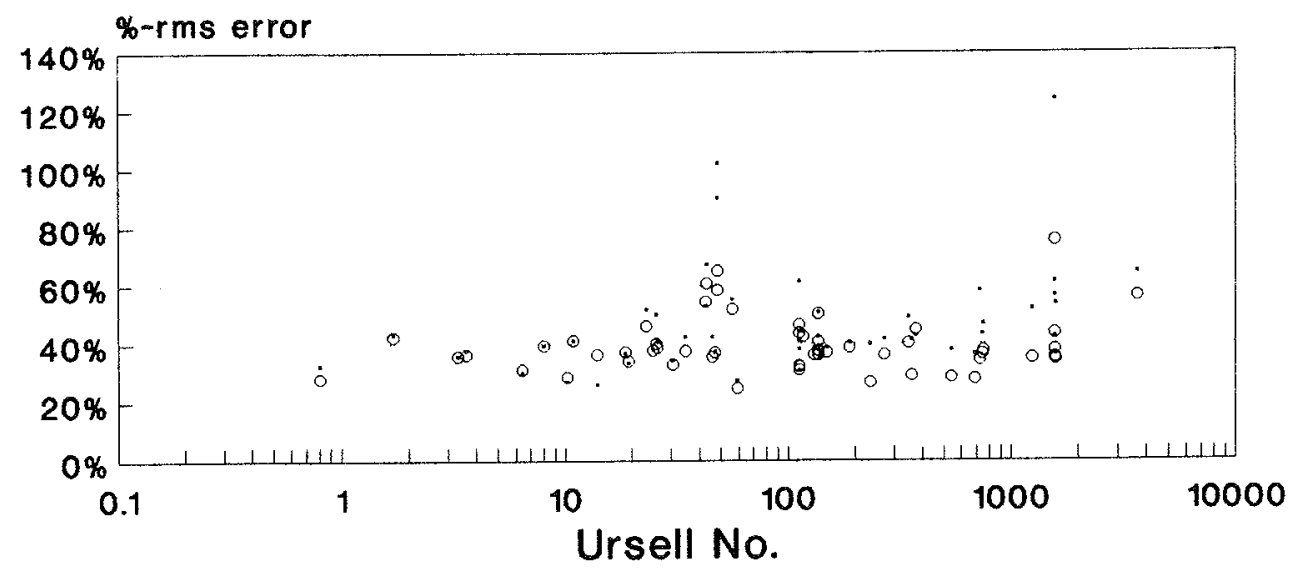

Rayleigh,z=mid-depth $\quad$ Beta-R, zamid-depth

(a)

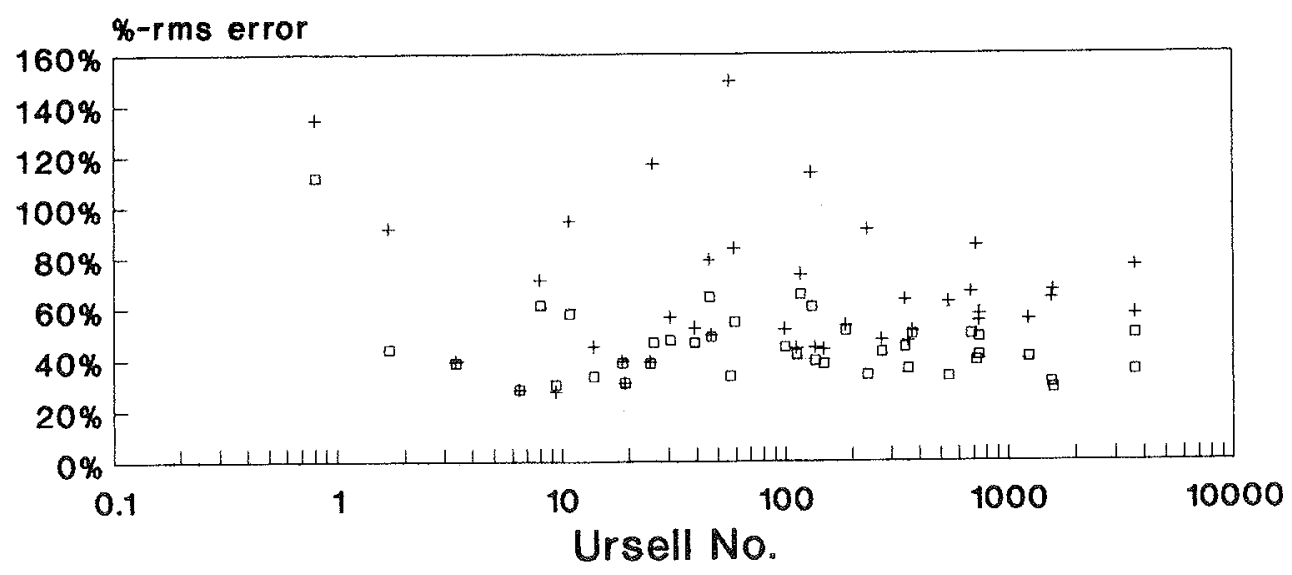

+ Rayleigh,zabottom a Beta-R, $z$ \&bottom

(b)

Figure 58: \%-Root-mean-square Error, Beta-Rayleigh Distribution, Negative Vertical Direction. (a) Mid-depth; (b) Near Bottom 


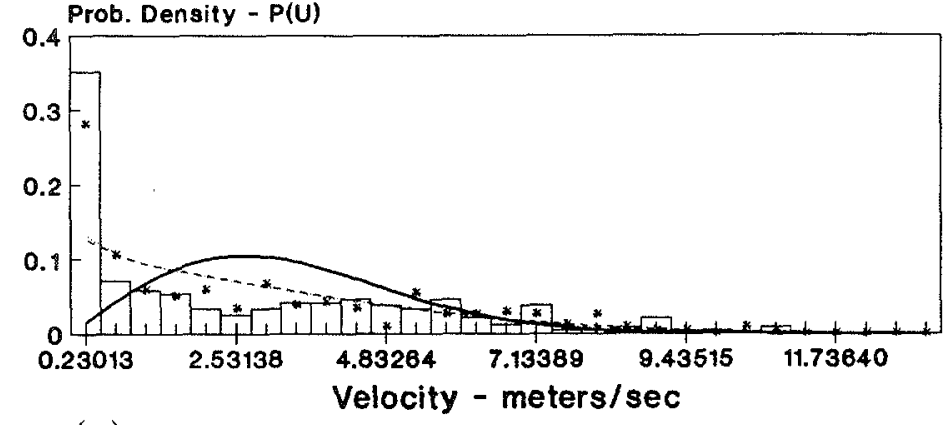

(a)

홍

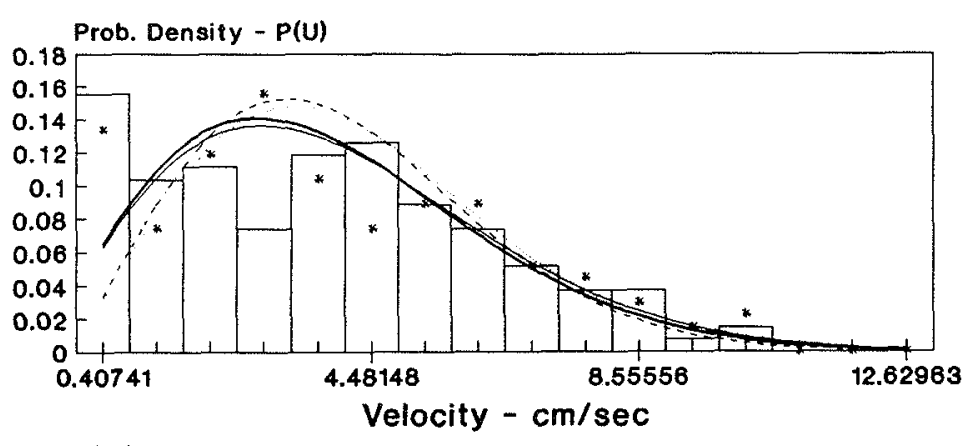

(b)

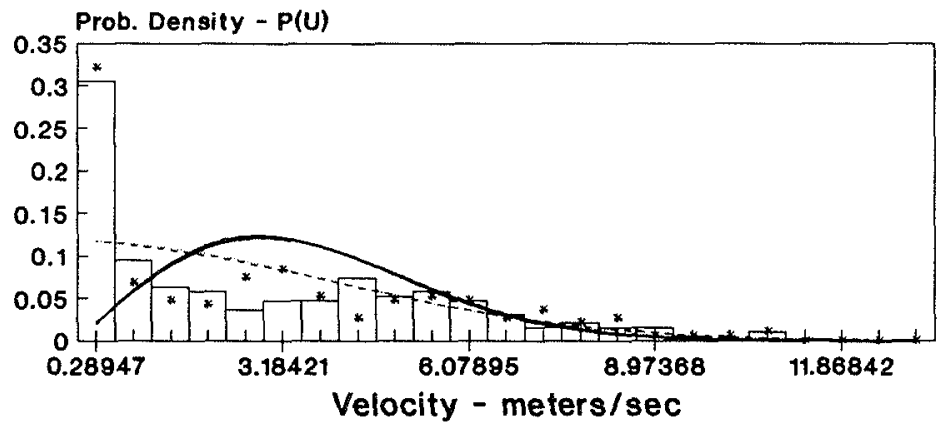

(d)

$\begin{array}{rrr}\square \text { Moseured Dist. (*) } & \text { Raylelgh Dist. (*) } & - \text { Bata-Rayleigh (*) } \\ * \quad \text { Mesaured Dist. (-) } & \text { Raylelgh Dist. (-) }\end{array}$

Figure 59: The Effect Of Varying Filter Width, Horizontal Velocity Peaks, Time Series No. 170. (a) Raw Data; (b) Filtered Data [0.20-5.00] s; (c) Filtered Data $[0.20-10.00] \mathrm{s} ;$ (d) Filtered Data $[0.10-5.00] \mathrm{s}$ 


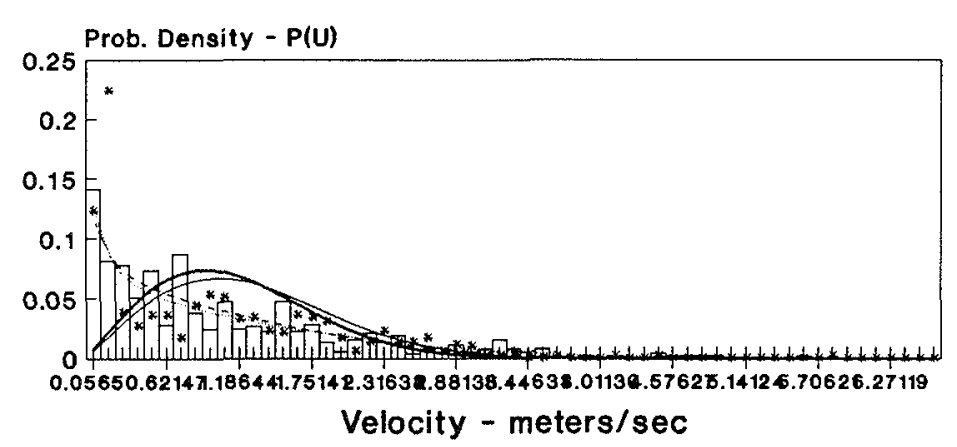

(a)

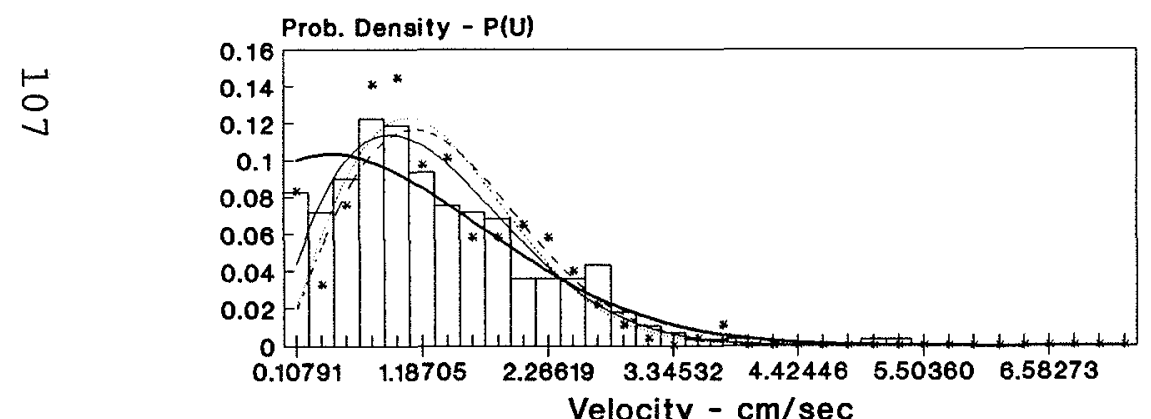

(c)

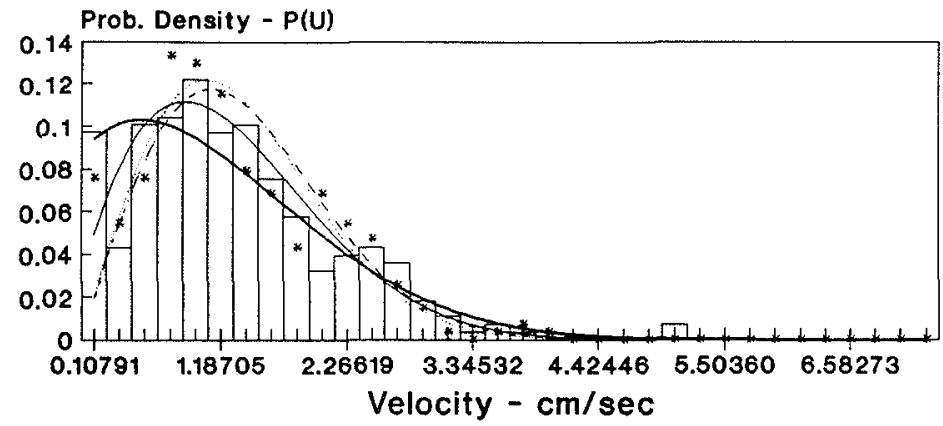

(b)

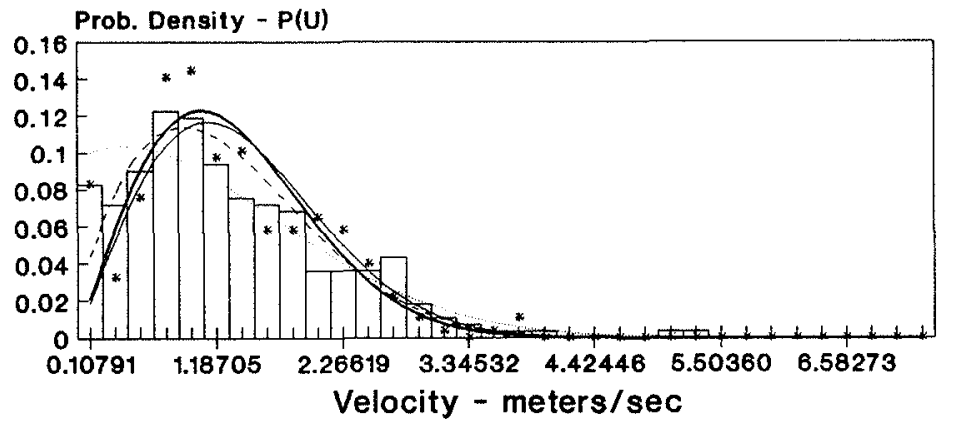

(d)

$$
\square \text { Measured Dist. (+) - Rayleigh Dist. (+) } \begin{array}{r}
\text { Beta-Rayleigh (+) } \\
* \text { Measured Dlet. (-) }- \text { Rayleigh Dist. (-) }
\end{array}
$$

Figure 60: The Effect of Varying Filter Width, Horizontal Velocity Peaks, Time Series No. 19. (a) Raw Data; (b) Filtered Data [0.20-5.00] s; (c) Filtered Data [0.20-10.00] s; (d) Filtered Data [0.10-5.00] s 


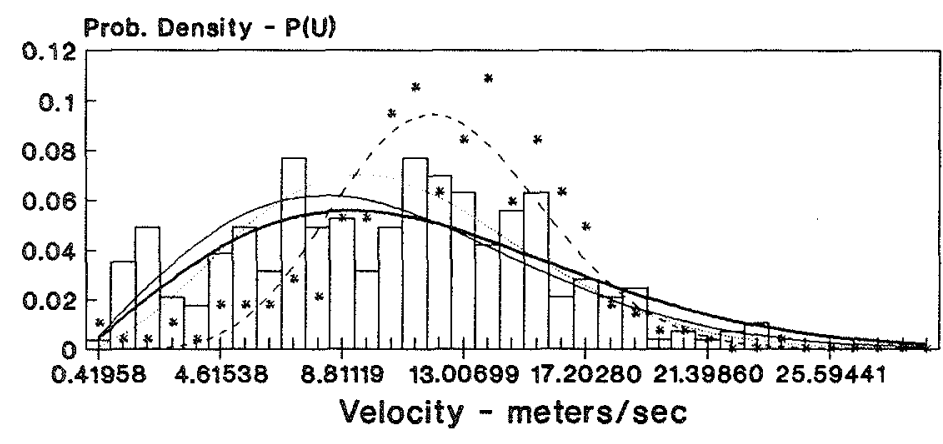

(a)

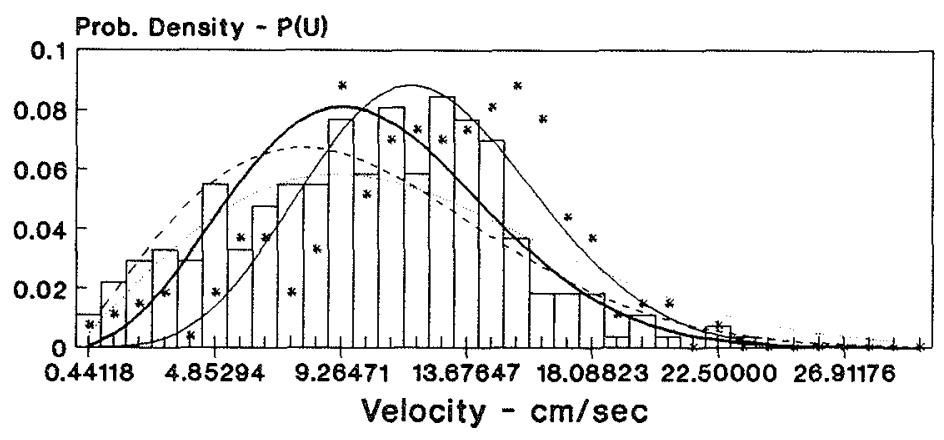

(b)

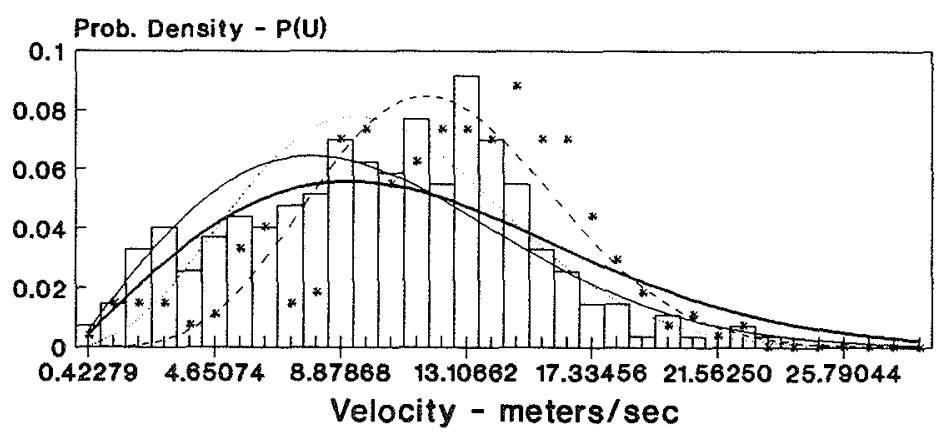

(d) (c)

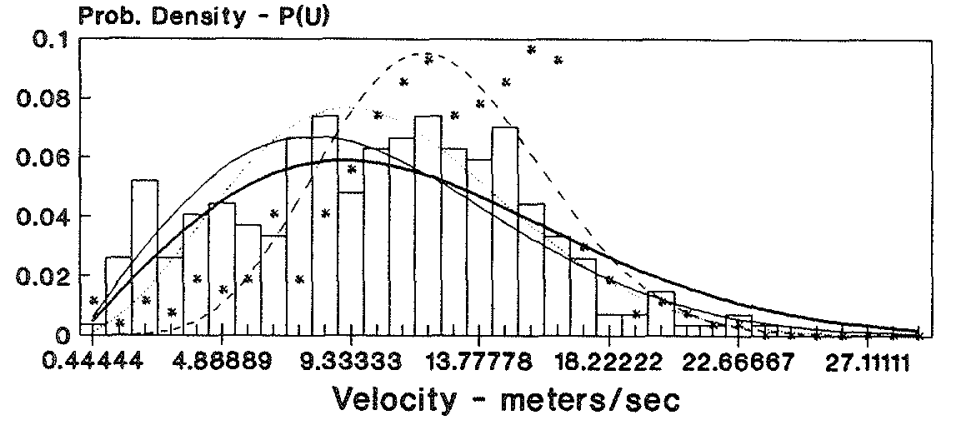

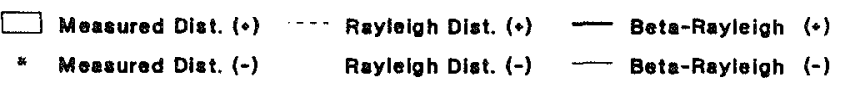

Figure 61: The Effect of Varying Filter Width, Horizontal Velocity Peaks, Time Series No. 70. (a) Raw Data; (b) Filtered Data [0.20-5.00] s; (c) Filtered Data [0.20-10.00] s; (d) Filtered Data [0.10-5.00] s 


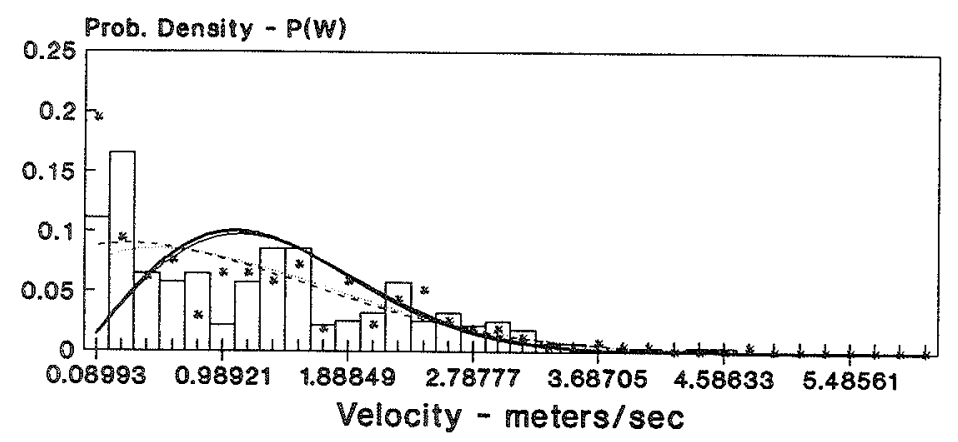

(a)

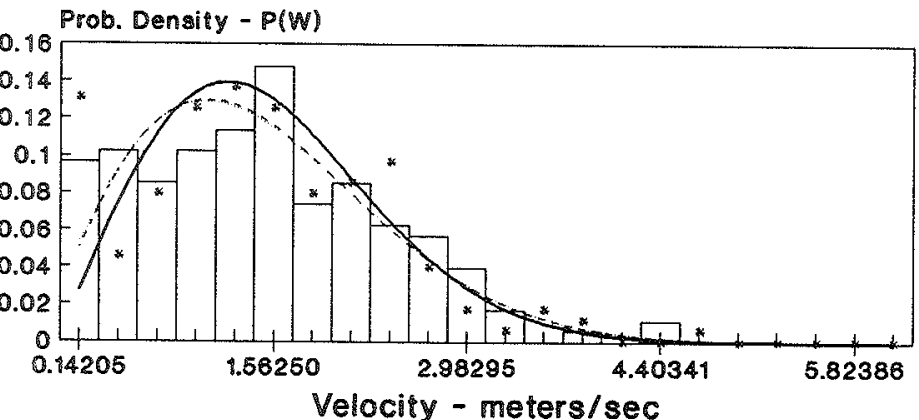

(d)

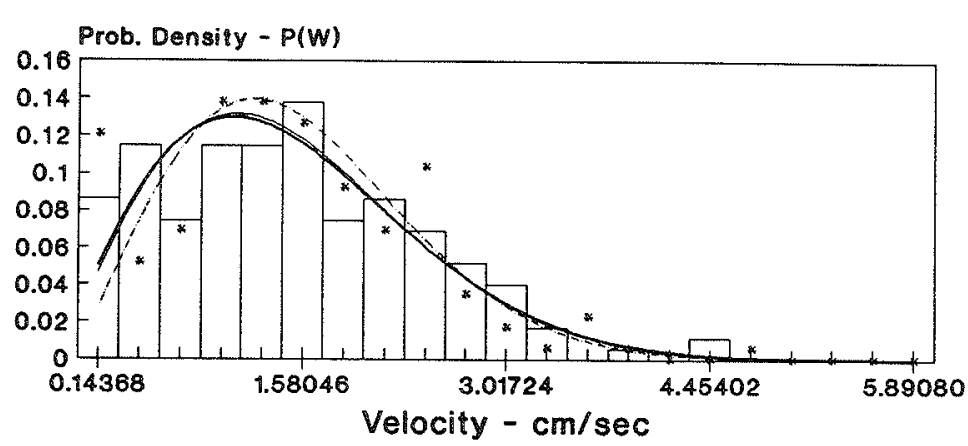

(b)

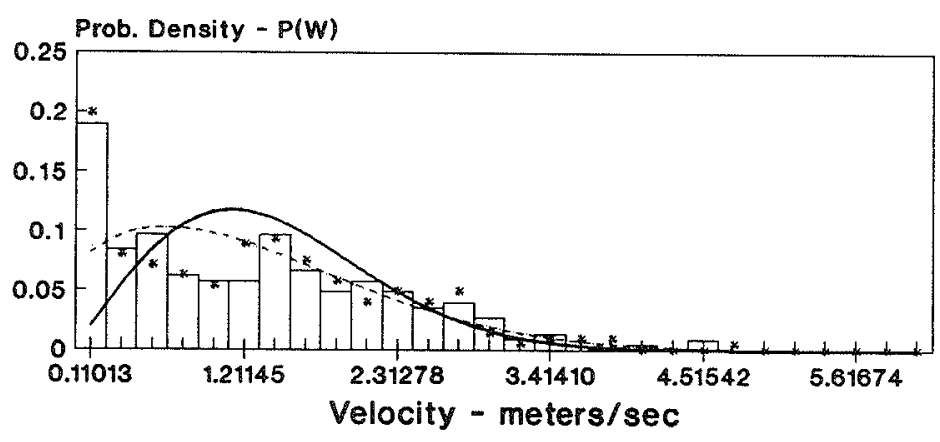

(c)

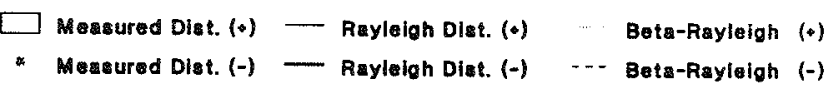

Figure 62: The Effect of Varying Filter Width, Vertical Velocity Peaks, Time Series No. 170. (a) Raw Data; (b) Filtered Data [0.20-5.00] s; (c) Filtered Data [0.20-10.00] s; (d) Filtered Data [0.10-5.00] s 


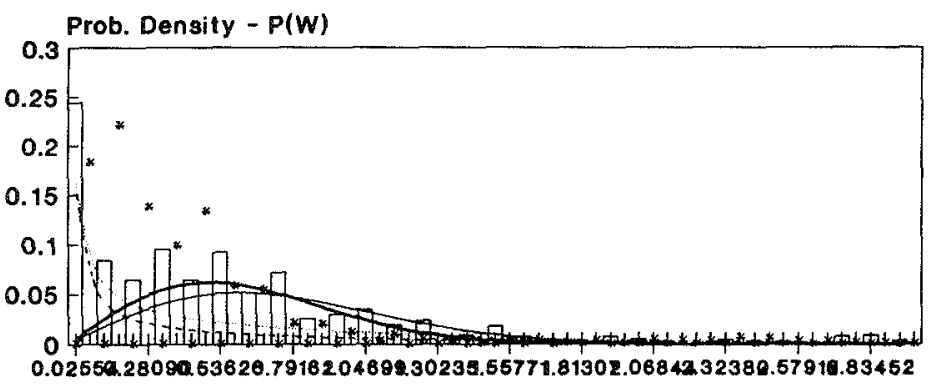

Velocity - meters/sec

(a)

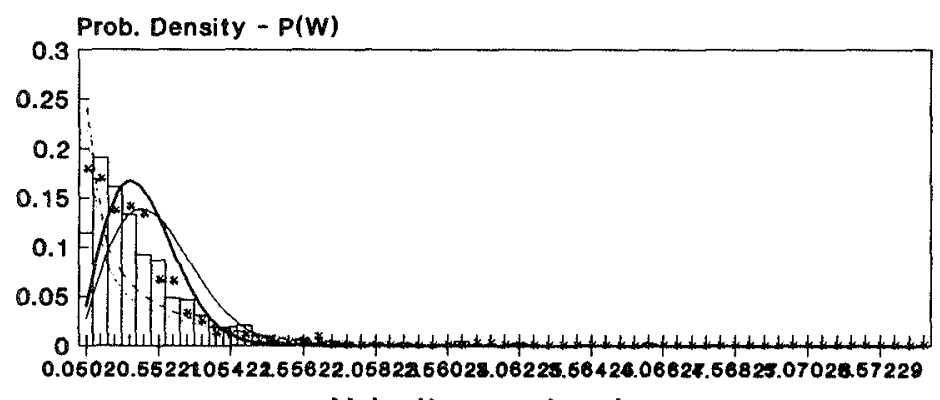

$$
\text { Velocity - meters/sec }
$$

(c)

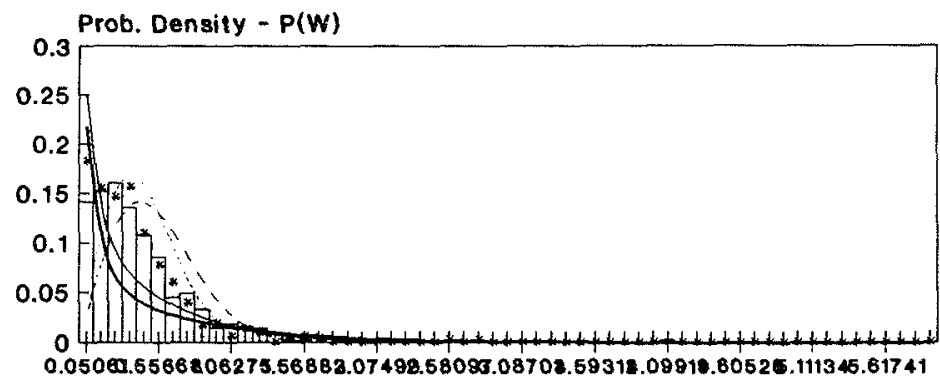

Velocity $-\mathrm{cm} / \mathrm{sec}$

(b)

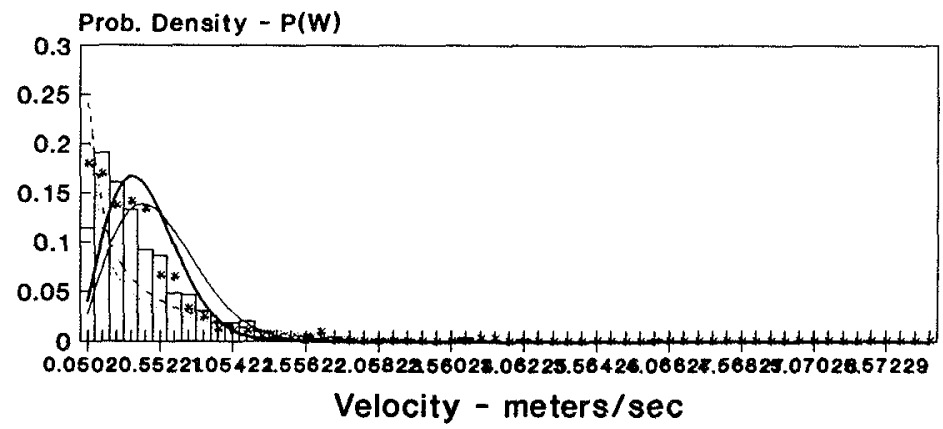

(d)

\footnotetext{
$\square$ Measured Dist. (*) 一 Raylelgh Dist. (*)

Beta-Raylaigh (*)

* Measured Dist. (-) - Rayleigh Dist. (-) -.. Beta-Rayleigh (-)
}

Figure 63: The Effect of Varying Filter Width, Vertical Velocity Peaks, Time Series No. 19. (a) Raw Data; (b) Filtered Data [0.20-5.00] s; (c) Filtered Data [0.20-10.00] s; (d) Filtered Data [0.10-5.00] s 


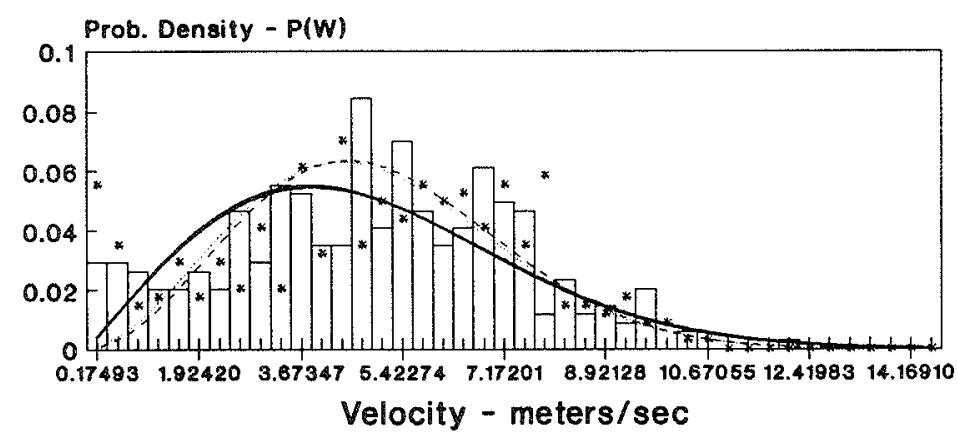

(a)

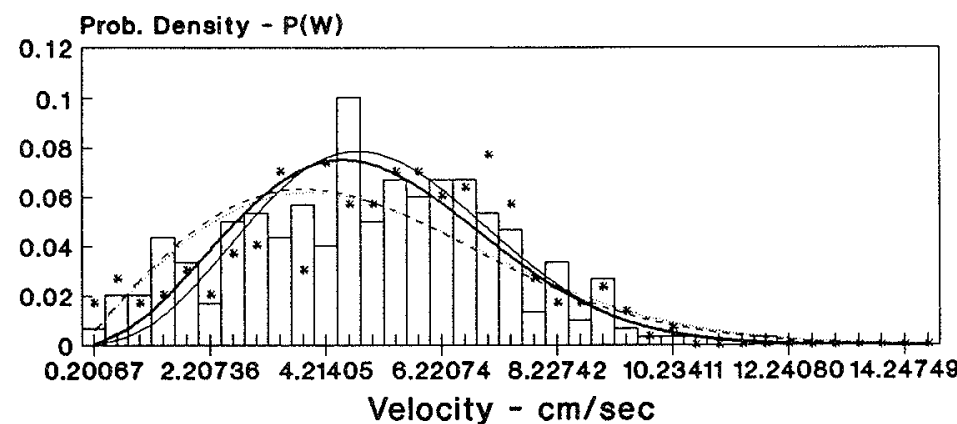

(b)

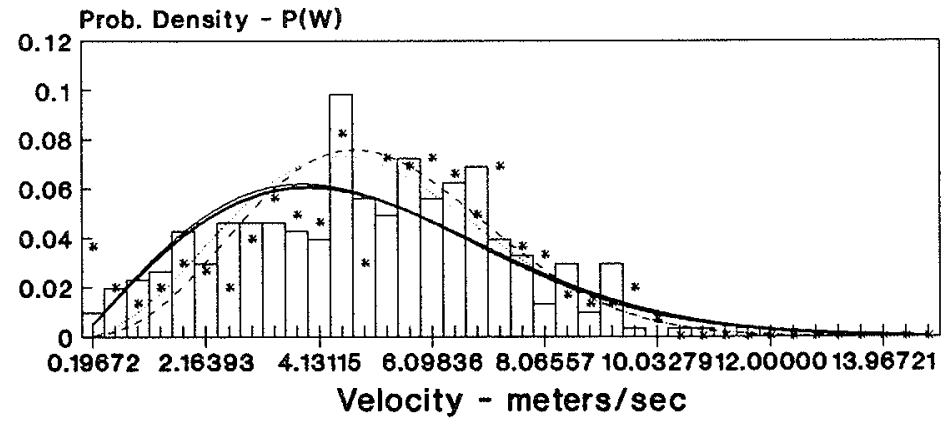

(d)

(c)

$\square$ Measured Dist. (+)
* Measured Dist. (-)

Figure 64: The Effect of Varying Filter Width, Vertical Velocity Peaks, Time Series No. 70. (a) Raw Data; (b) Filtered Data [0.20-5.00] s; (c) Filtered Data [0.20-10.00] s; (d) Filtered Data [0.10-5.00] s 


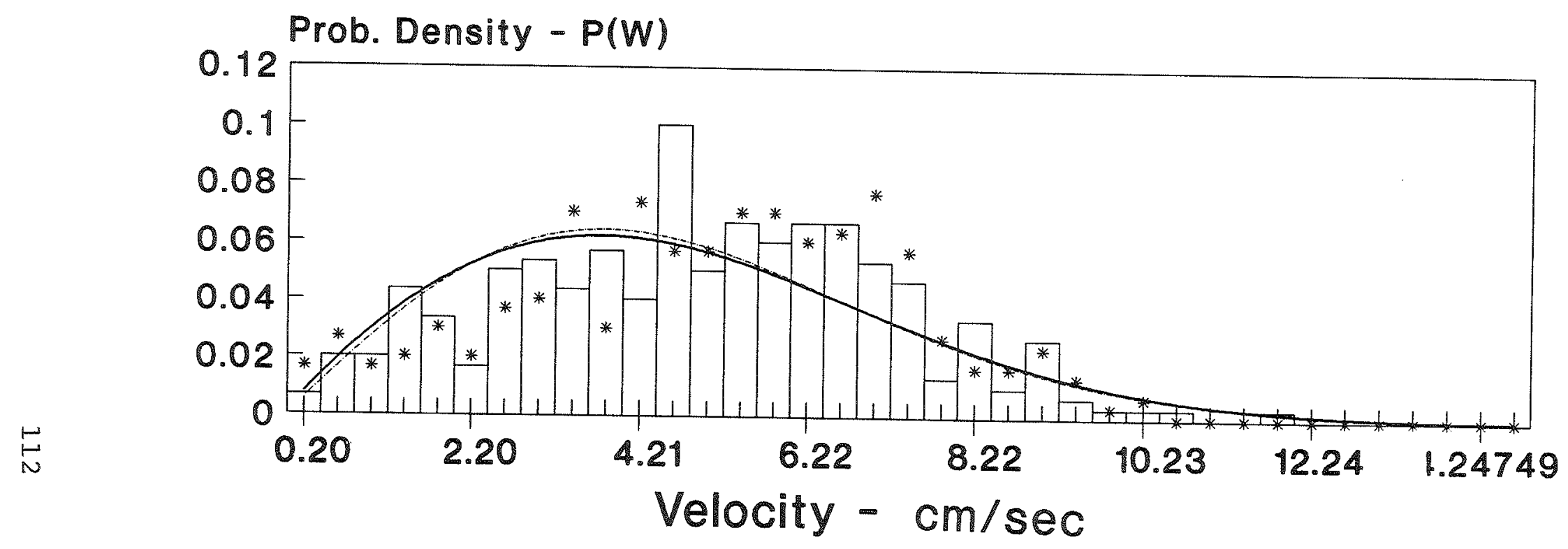

* Measured Dist. (t) ….. Rayleigh Dist. (t) - Beta-Rayleigh (t)

* Measured Dist. (-) Rayleigh Dist. (-) - Beta-Rayleigh (-)

Figure 65: Vertical Velocity Peaks $w_{r m s}$ only Parameter 


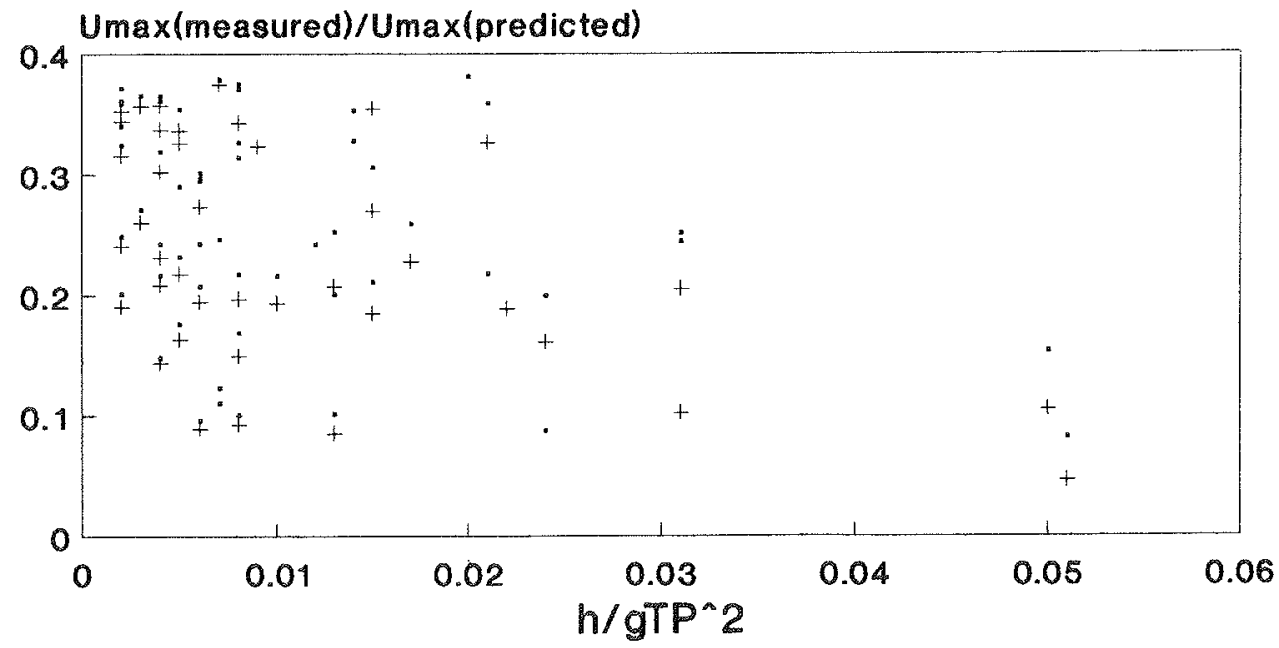

$U, z^{\text {mid-depth }}+\mathrm{U}$, zanear bottom

(a) Horizontal Direction

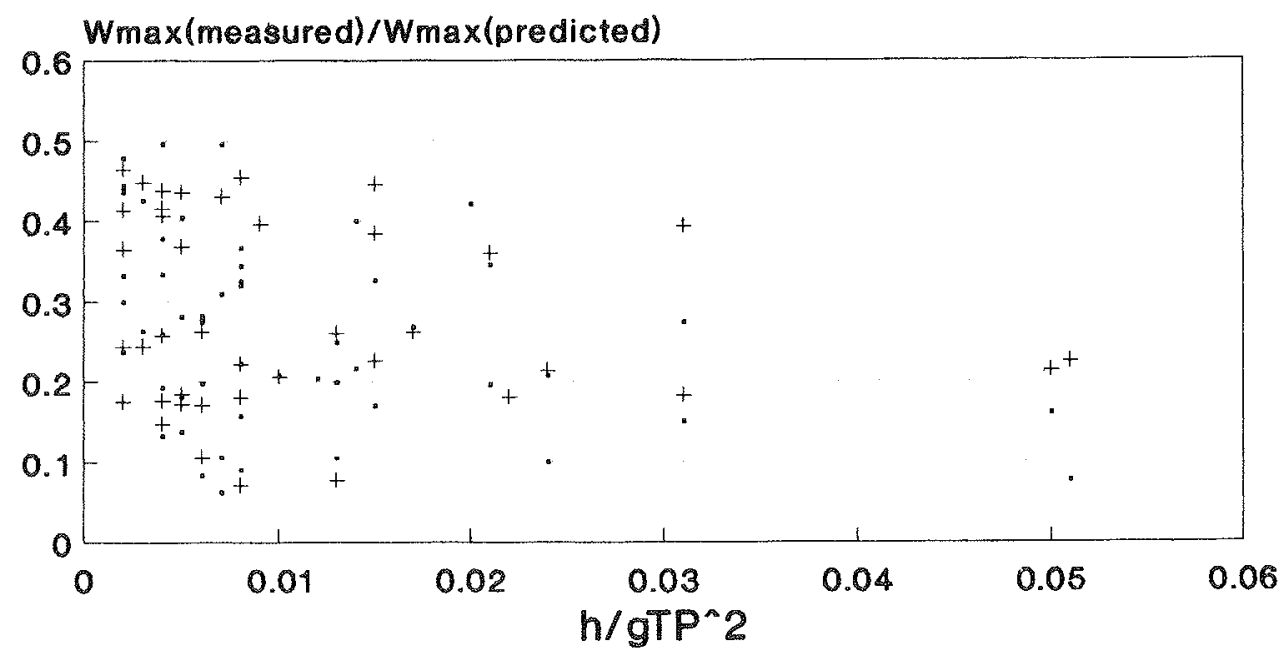

- W, zmidudepth + W, zønear bottom

(b) Vertical Direction

Figure 66: Maximum Velocity Measured/Predicted vs. Relative Depth. (a) Horizontal Direction; (b) Vertical Direction 


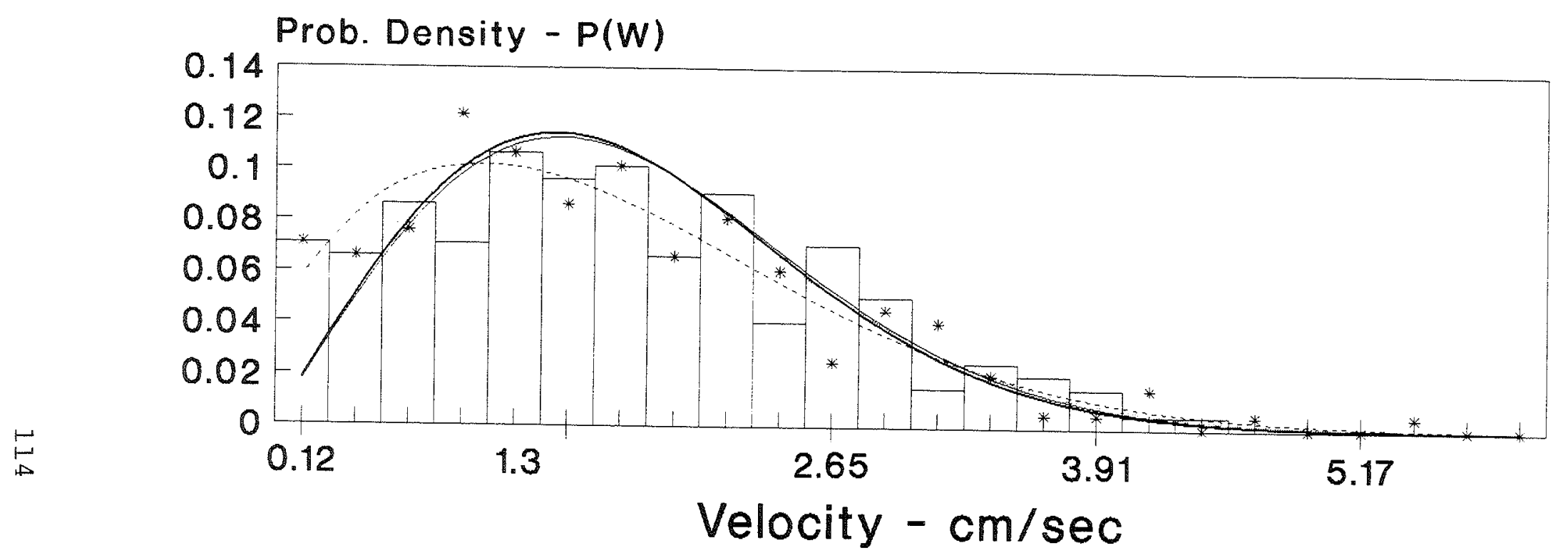

\begin{tabular}{llll}
\hline Measured Dist. (+) & - Rayleigh Dist. (+) & Beta-Rayleigh (+) \\
$*$ & Measured Dist. (-) & - Rayleigh Dist. (-) & -....
\end{tabular}

Figure 67: Vertical Velocity Peaks Probability Distribution, 2x Usual $W_{\max }$ 


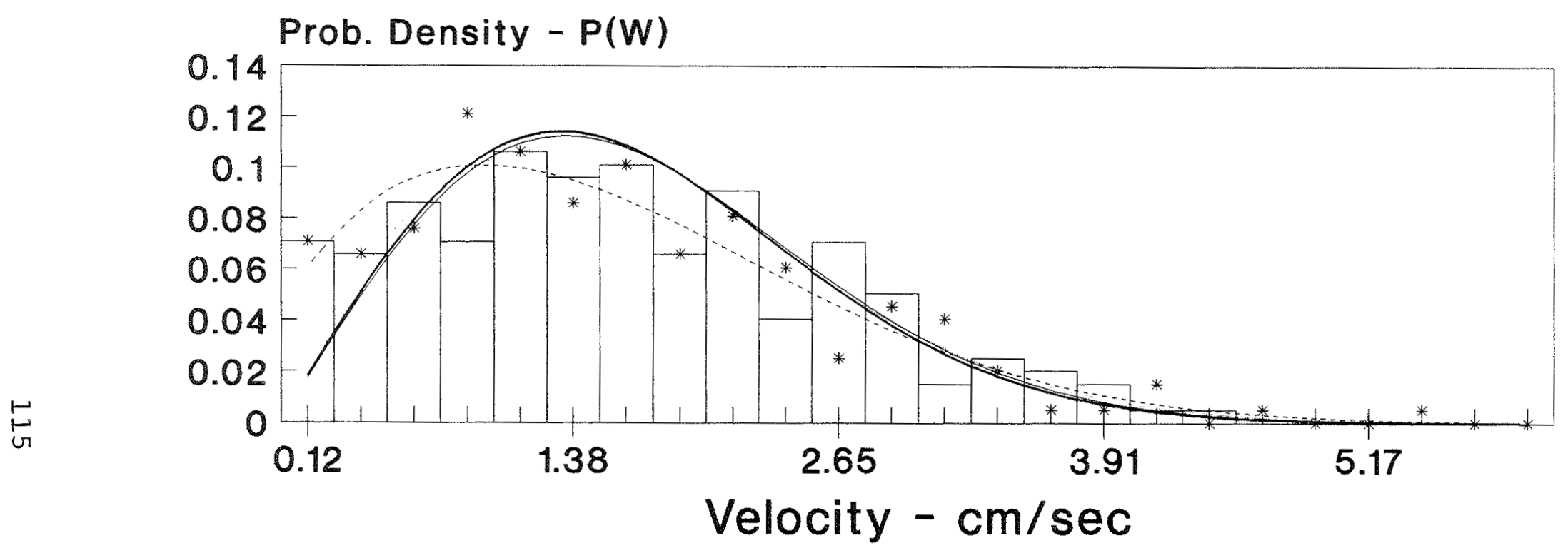

$\square$ Measured Dist. (+) - Rayleigh Dist. (+) Beta-Rayleigh (+)

* Measured Dist. (-) - Rayleigh Dist. (-) …... Beta-Rayleigh (-)

Figure 68: Vertical Velocity Peaks Probability Distribution, $0.5 x$ Usual $W_{\max }$ 


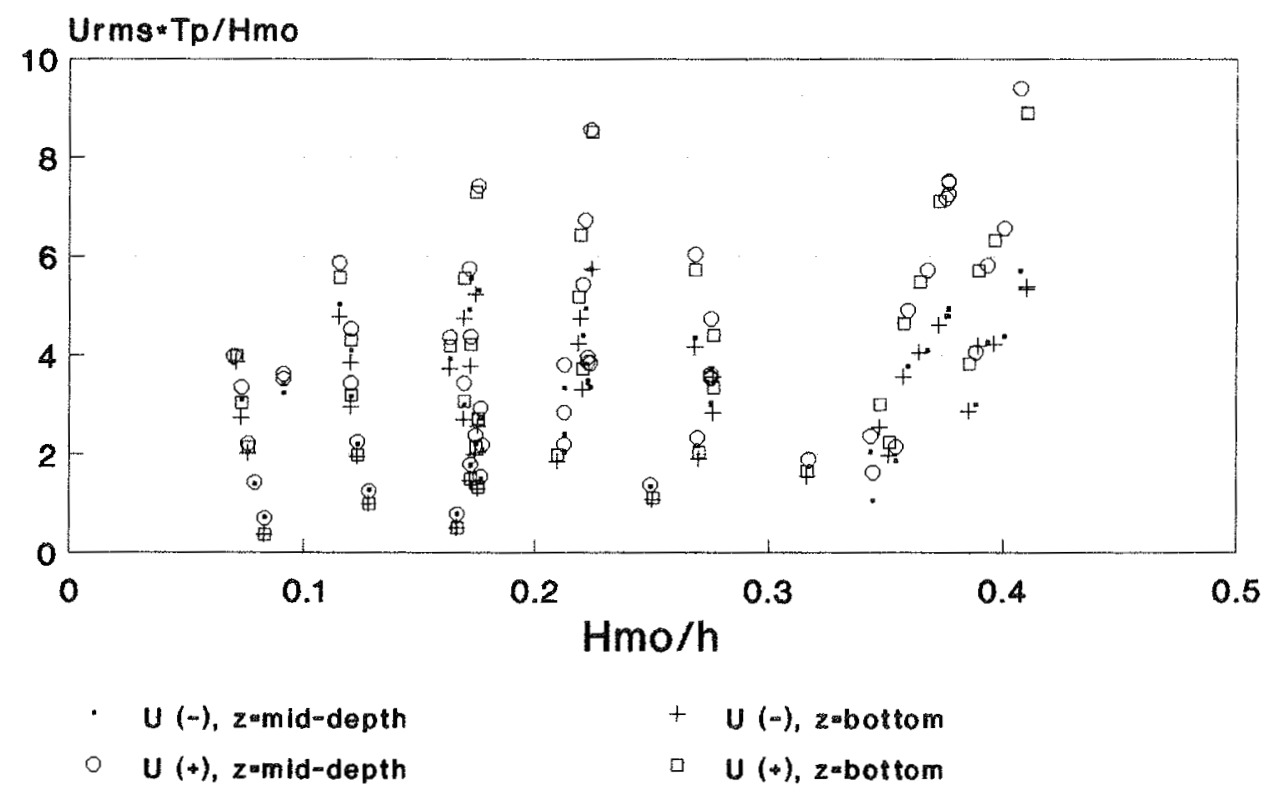

(a)

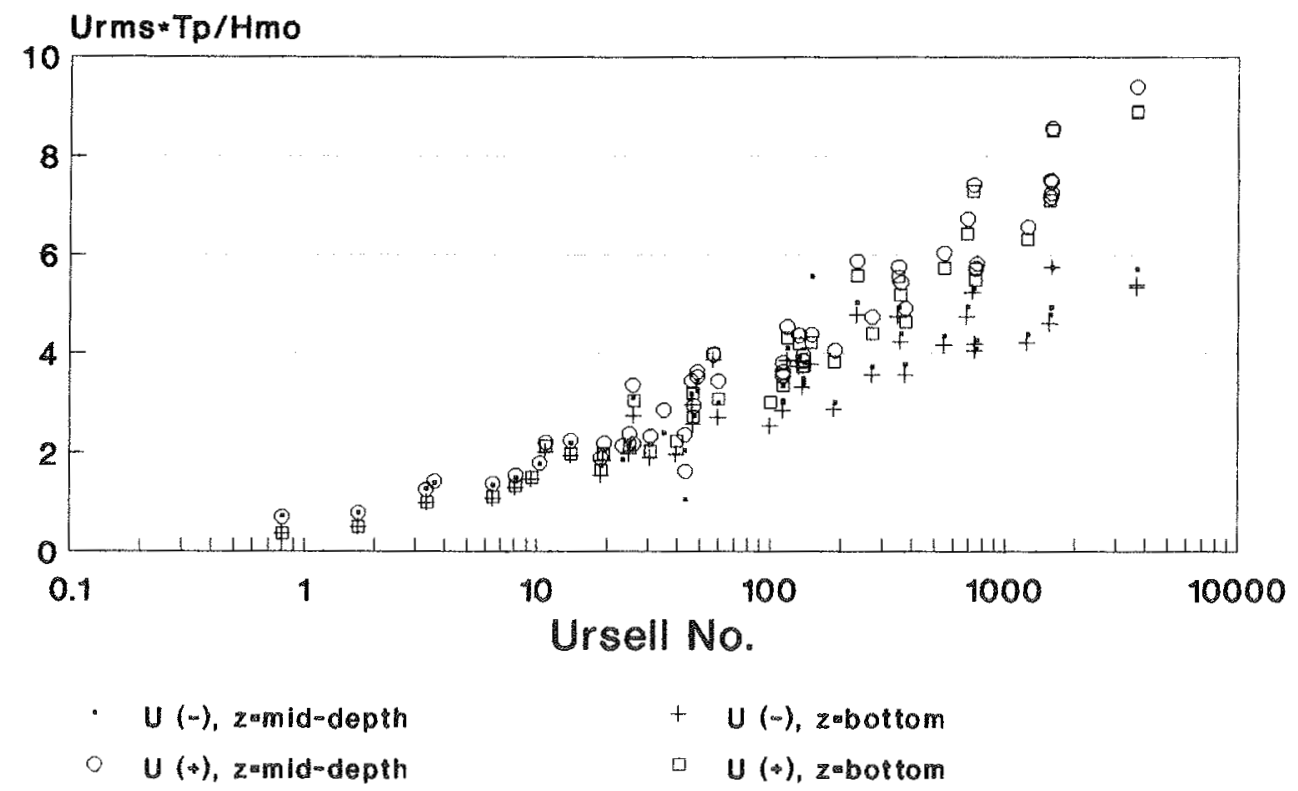

(b)

Figure 69: Parameterization of Beta-Rayleigh Distribution, $U_{r m s}$. (a) vs. $H_{m o} / h$; (b) vs. Ursell No. 


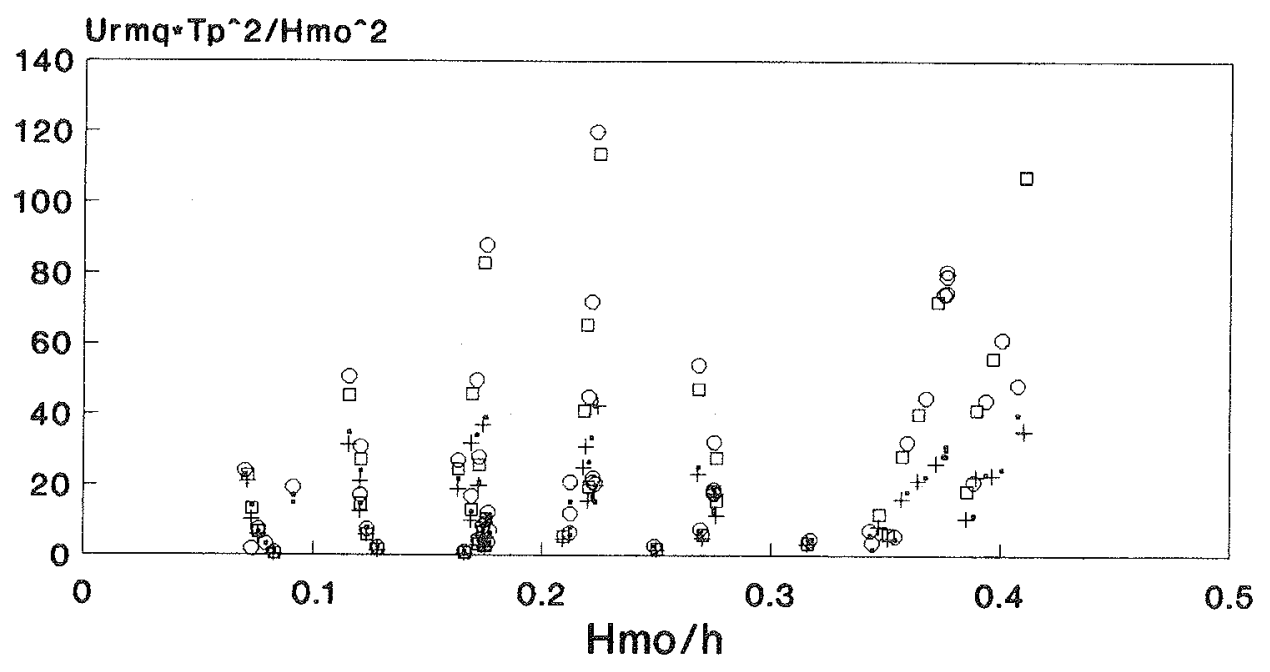
U (-), zamid-depth
$+\quad u(-), z \operatorname{zbot} t o m$
o $u(+), z=m i d-d e p t h$
D $U(\$), z$ bottom

(a)

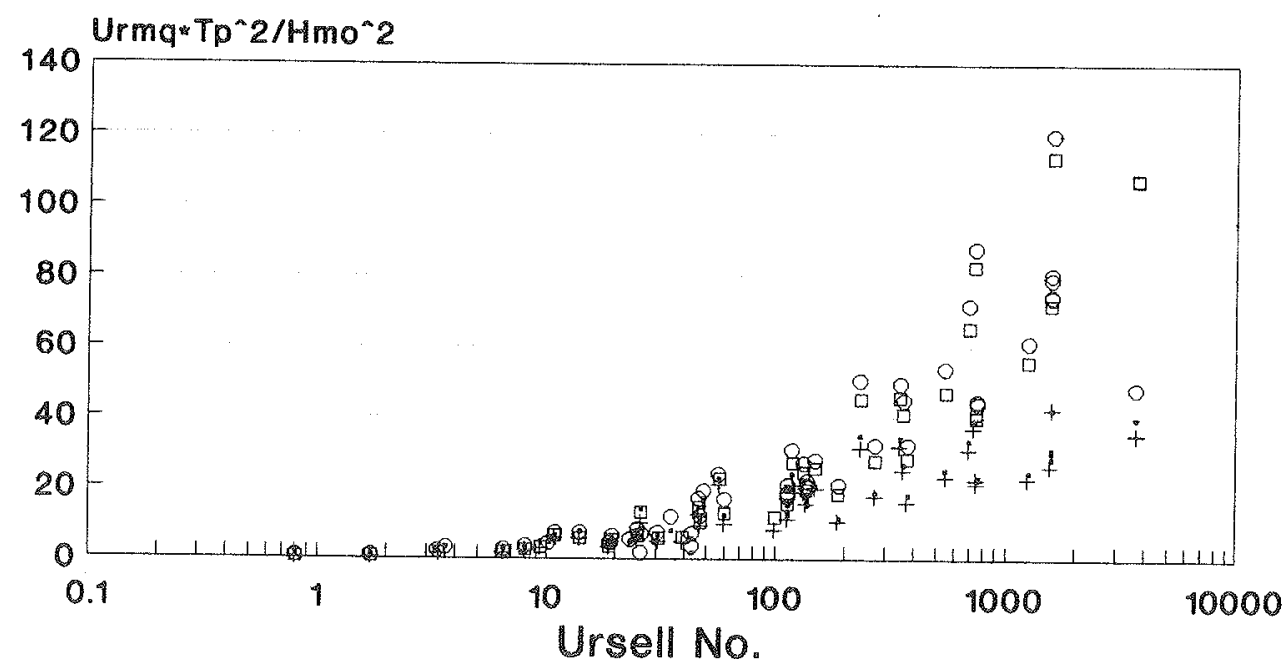

$\begin{array}{ll}U(-), z^{2 m i d-d e p t h} & +U(-), \text { zabottom } \\ U(\infty), z_{m} \text { mid-depth } & \square U(\phi), z \text { bottom }\end{array}$

(b)

Figure 70: Parameterization of Beta-Rayleigh Distribution, $U_{\text {rmq }}$. (a) vs. $H_{m o} / h_{;}$ (b) vs. Ursell No. 


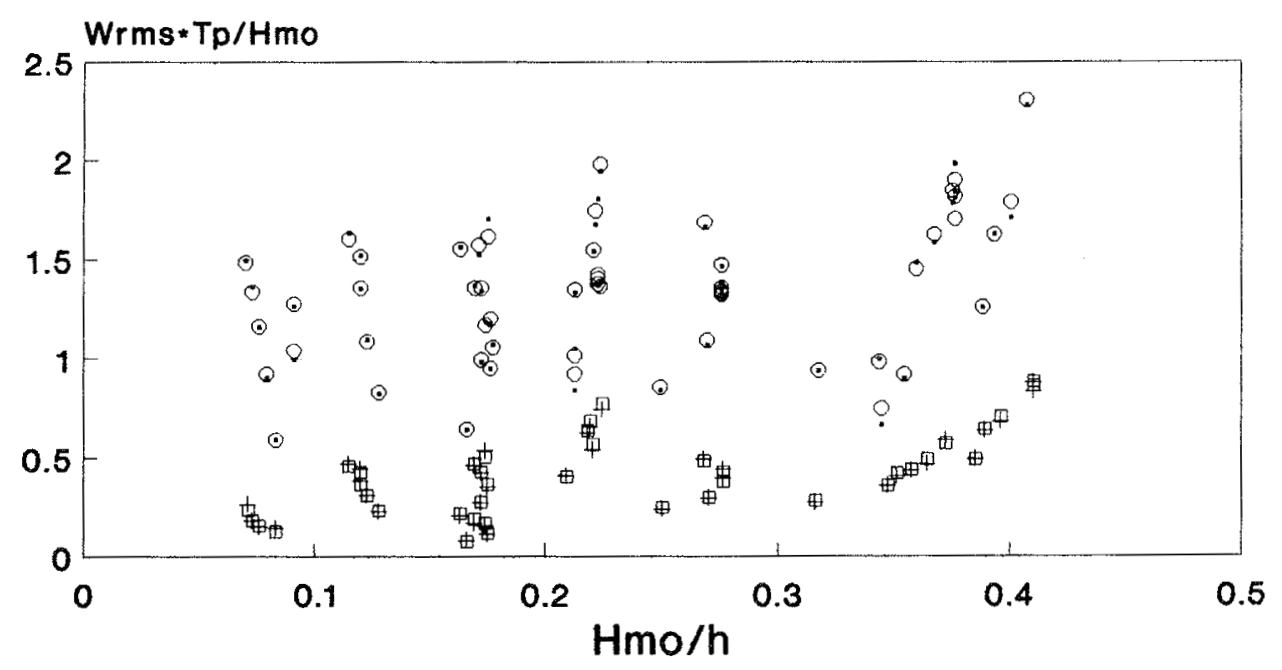
- W (+), z-mid-depth
$+W(+), z-b o t t o m$
o $W(-), z-m i d-d e p t h$
ㅁ $\quad w(-), z=b o t t o m$

(a)

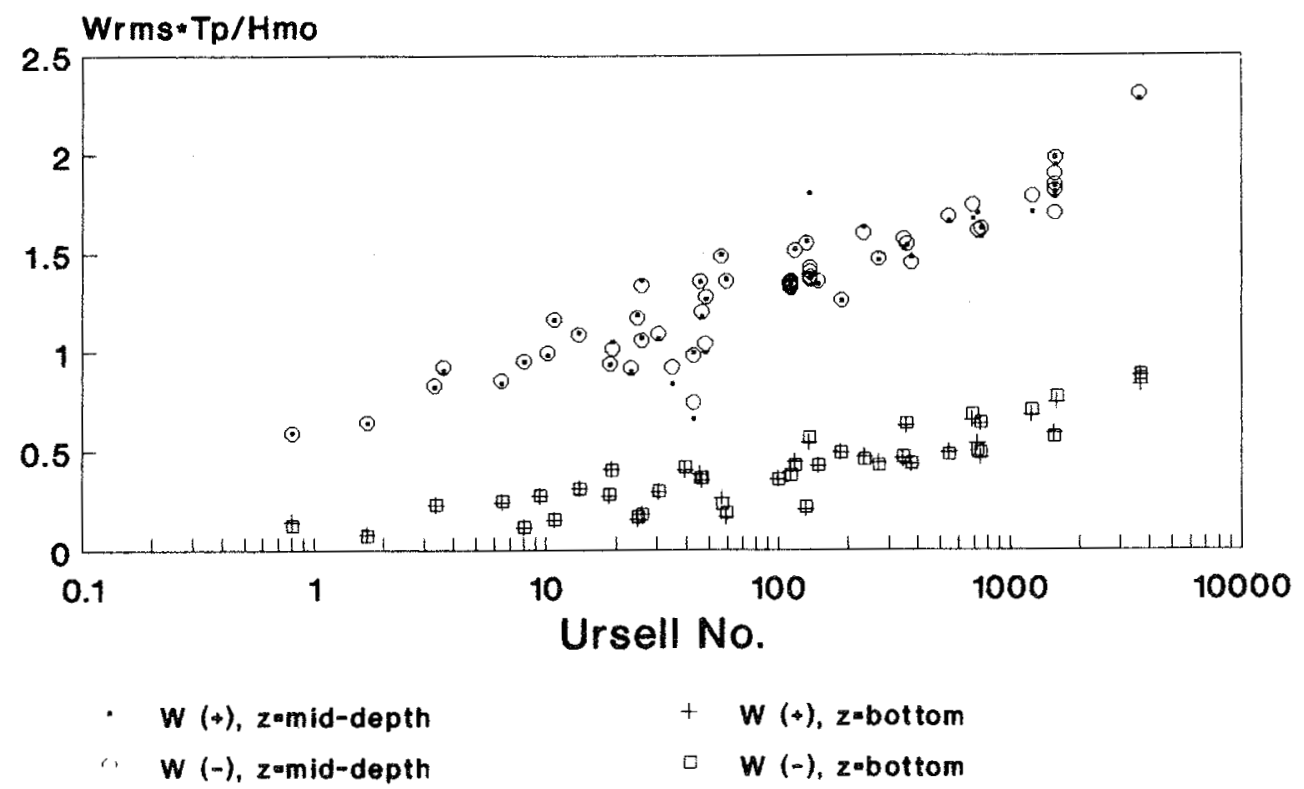

(b)

Figure 71: Parameterization of Beta-Rayleigh Distribution, $W_{r m s}$. (a) vs. $H_{m o} / h$; (b) vs. Ursell No. 


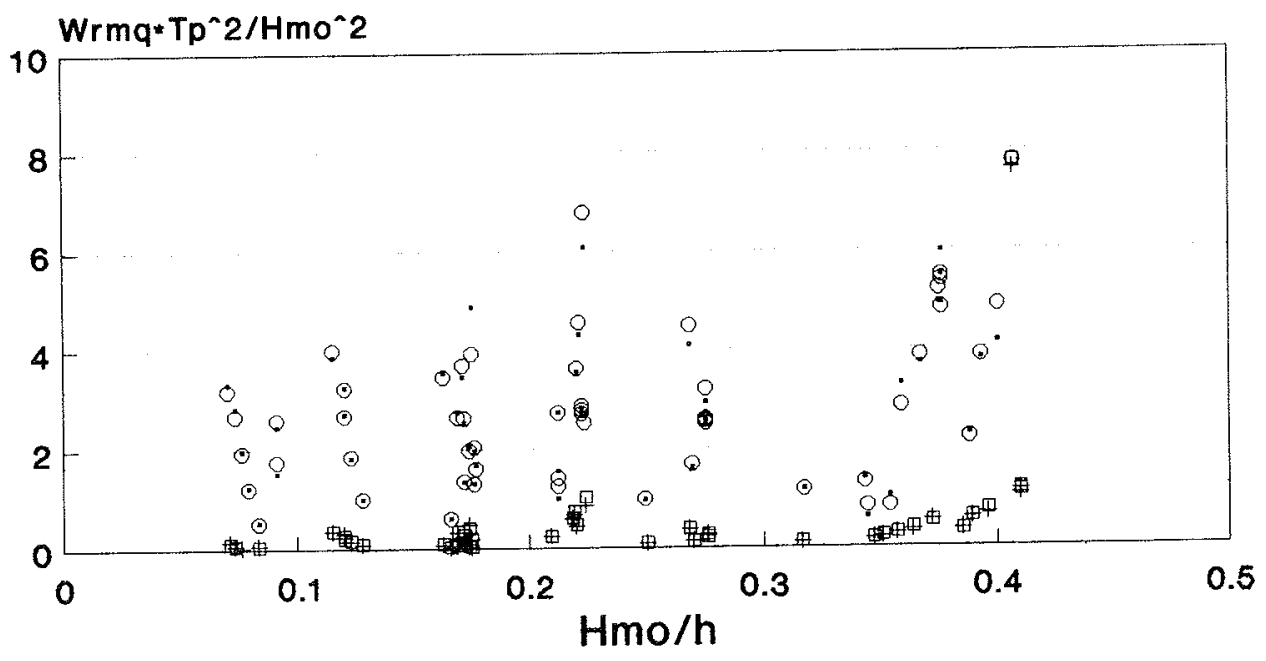

- $\quad W(+), z=$ mid-depth

$+W(+), z-b o t t o m$

o $W(-), z=m i d-d e p t h$

a $W(-), z=$ bottom

(a)

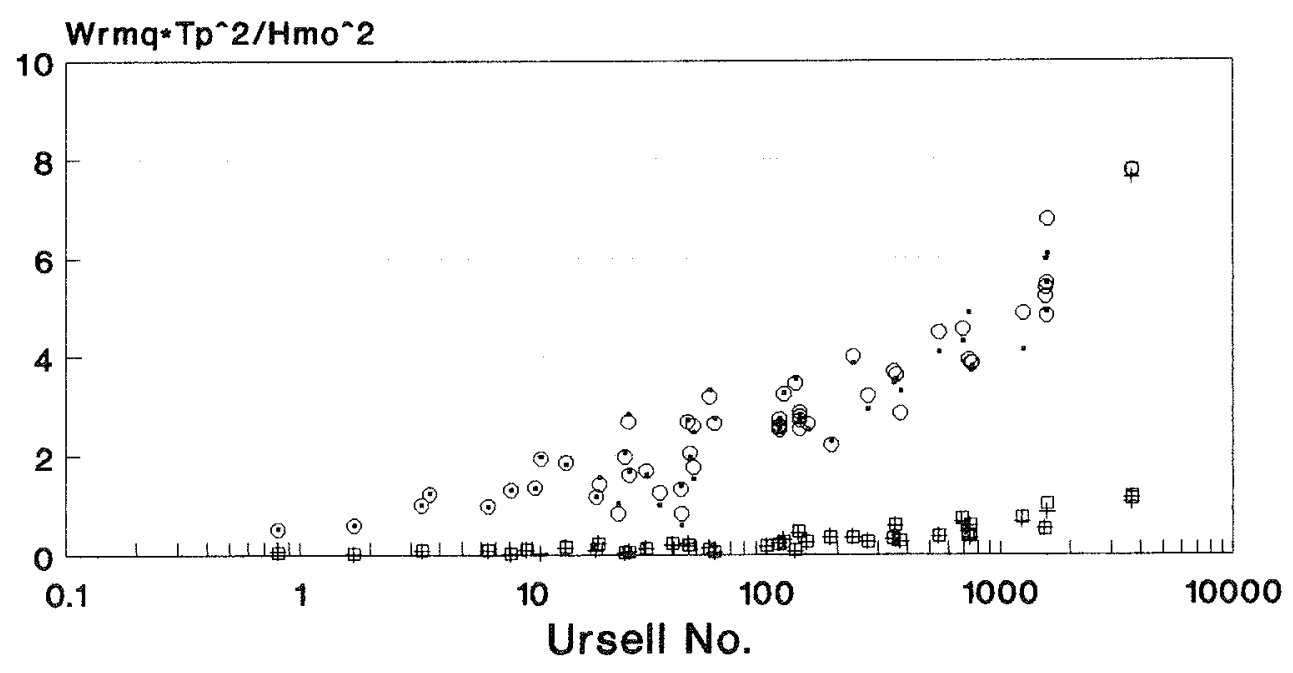

- W(t), z-mid-depth

$+\quad W(+), z=$ bottom

o $W(-), z$ mid-depth

ㅁ W (-), zabottom

(b)

Figure 72: Parameterization of Beta-Rayleigh Distribution, $W_{r m q}$. (a) vs. $H_{m o} / h$; (b) vs. Ursell No. 

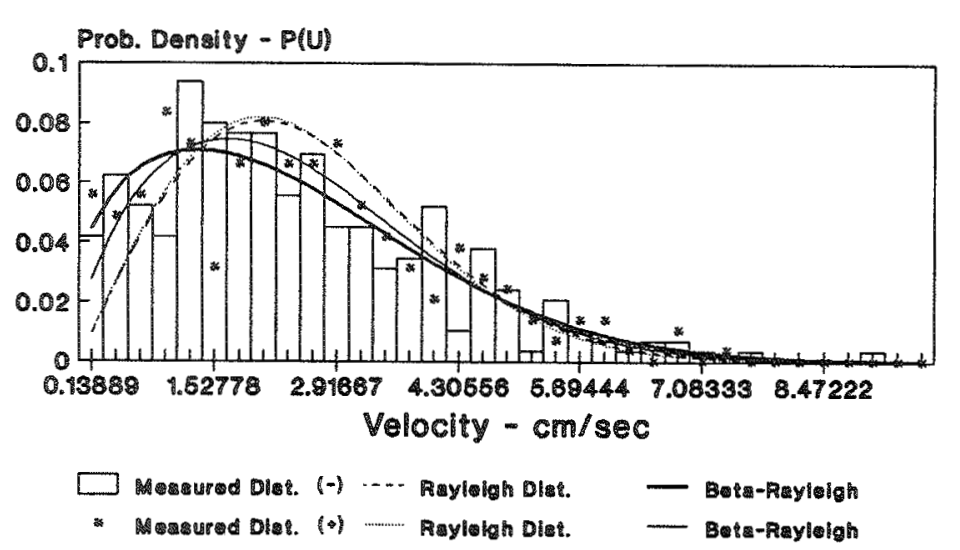

(a)
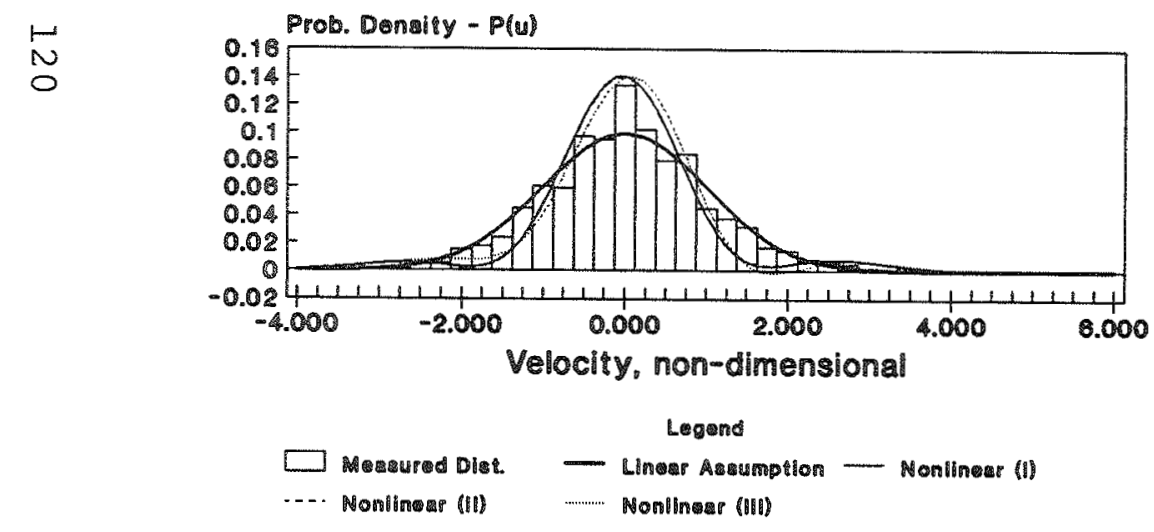

(c)

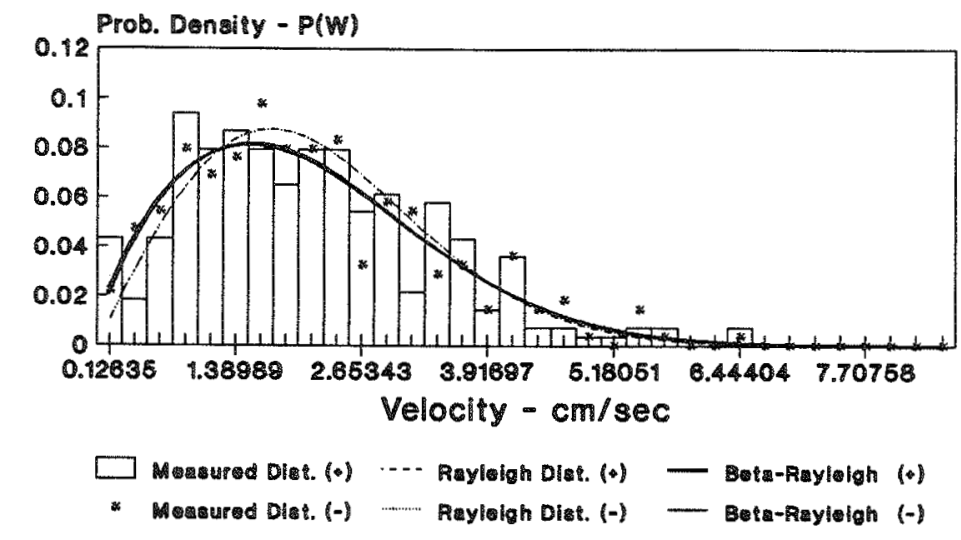

(b)
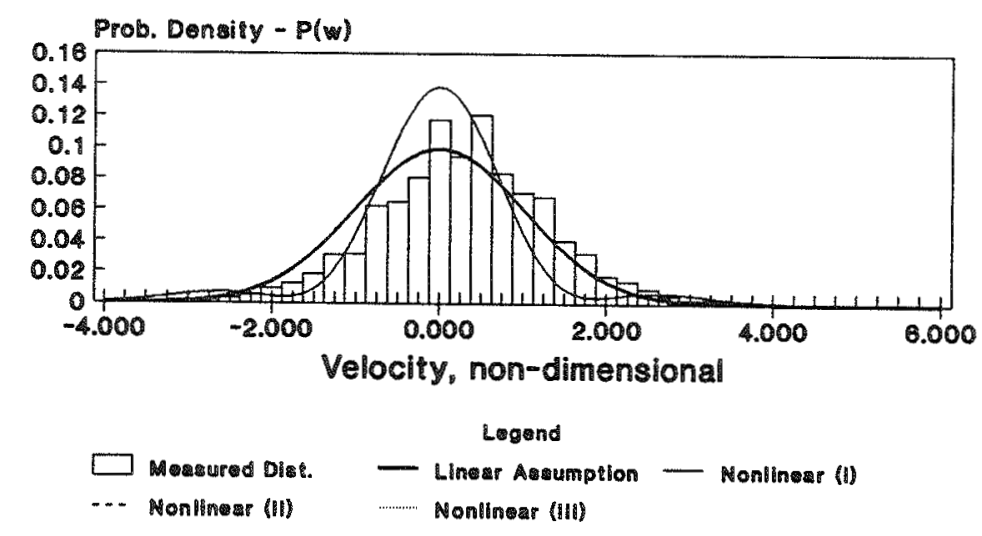

(d)

Figure 73: Time Series No. 9. (a) Filtered Data [0.20-5.00] s; (b) Filtered Data [0.20-5.00] s; (c) Raw Data; (d) Raw Data 


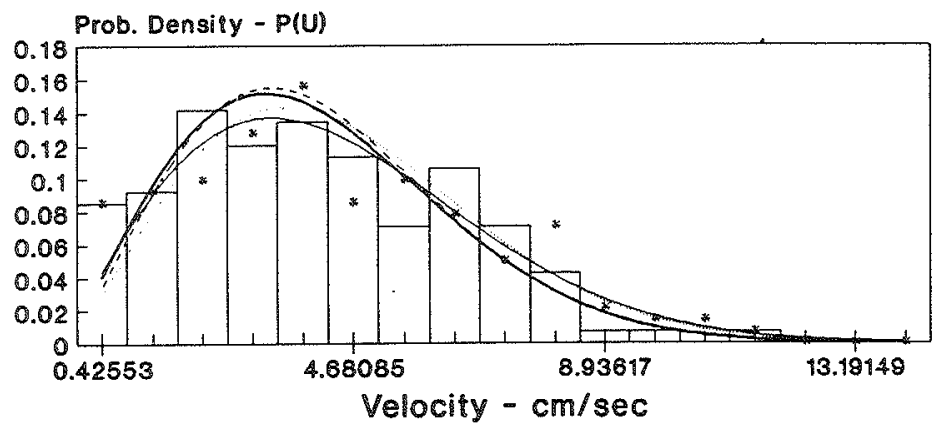

$\square$ Meseured Olat. (-) -..- Rayleigh Dist. $\quad$ - Bata-Aaylolgh

* Measured Dlat. (o) Rayloigh Dlat. Bats-Rayleigh

(a)

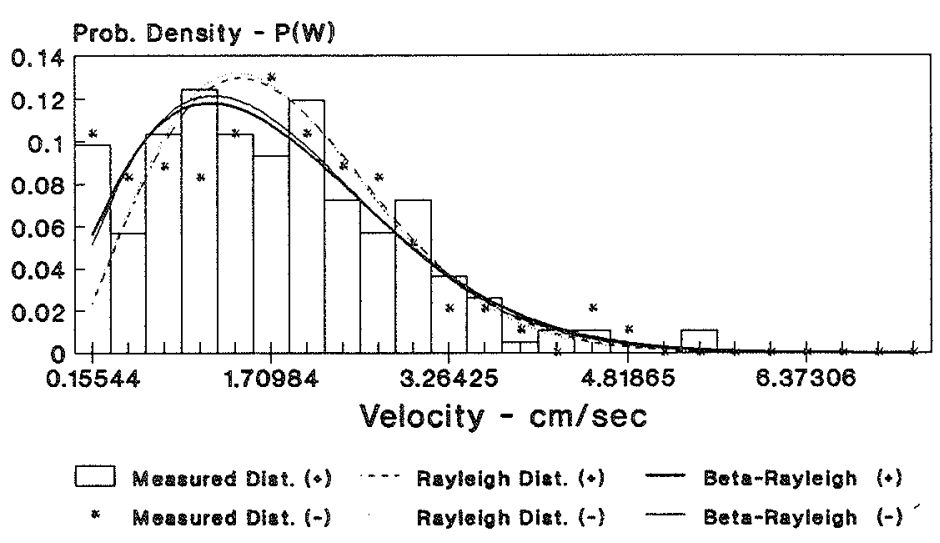

(b)
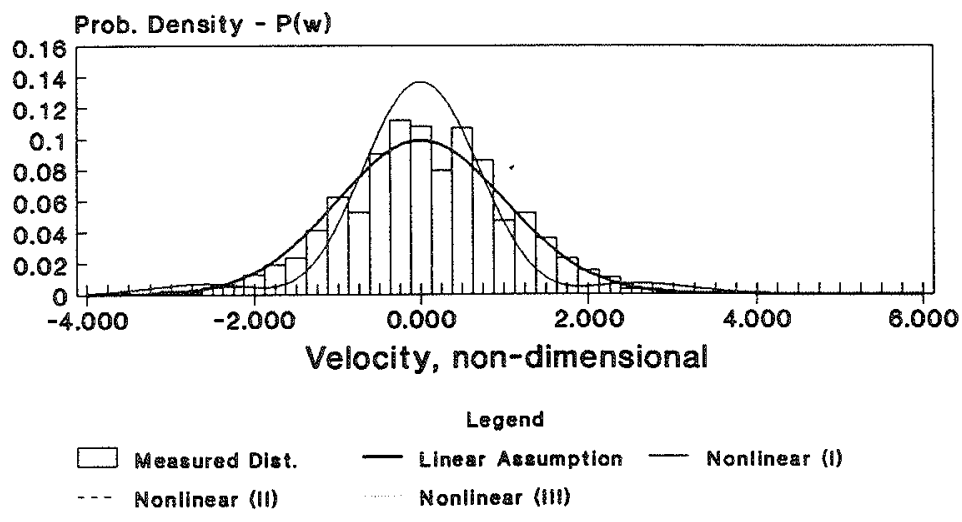

(d)

Figure 74: Time Series No. 153. (a) Filtered Data [0.20-5.00] s; (b) Filtered Data

[0.20-5.00] s; (c) Raw Data; (d) Raw Data 


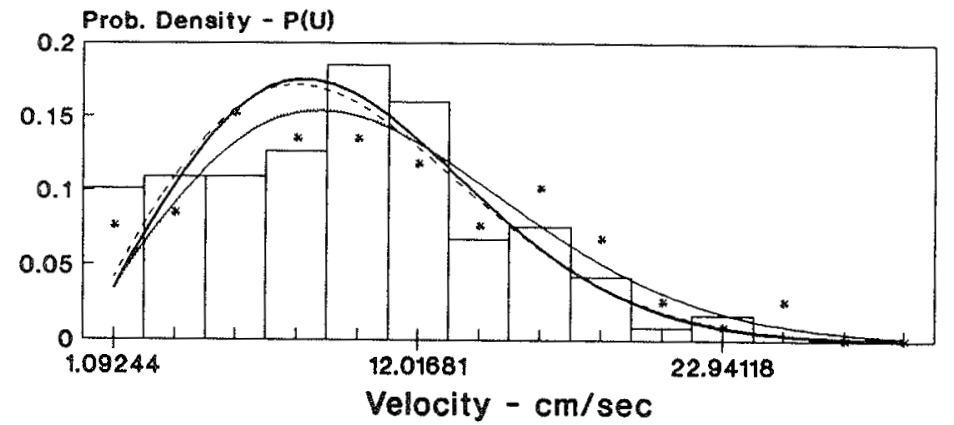

$\square$ Measured Dist. (-) -.. Rayleigh Dist. - Beta-Rayleigh * Mesaurod Dist. (*) … Rayleigh Dist. - Beta-Rayleigh

(a)
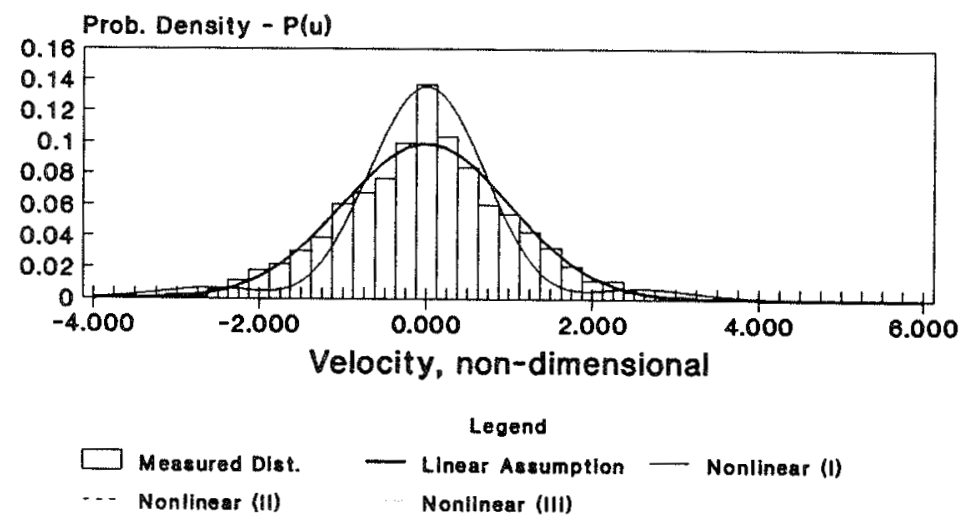

(c)

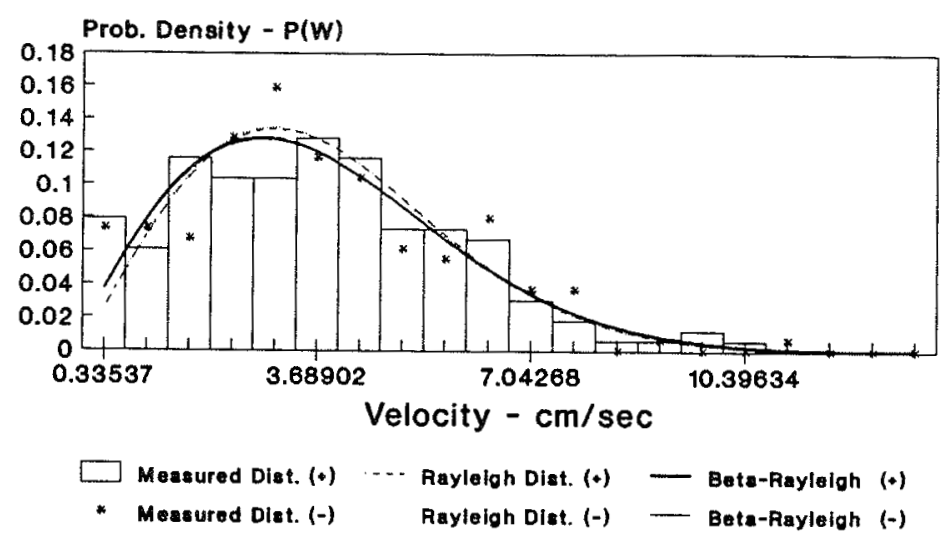

(b)
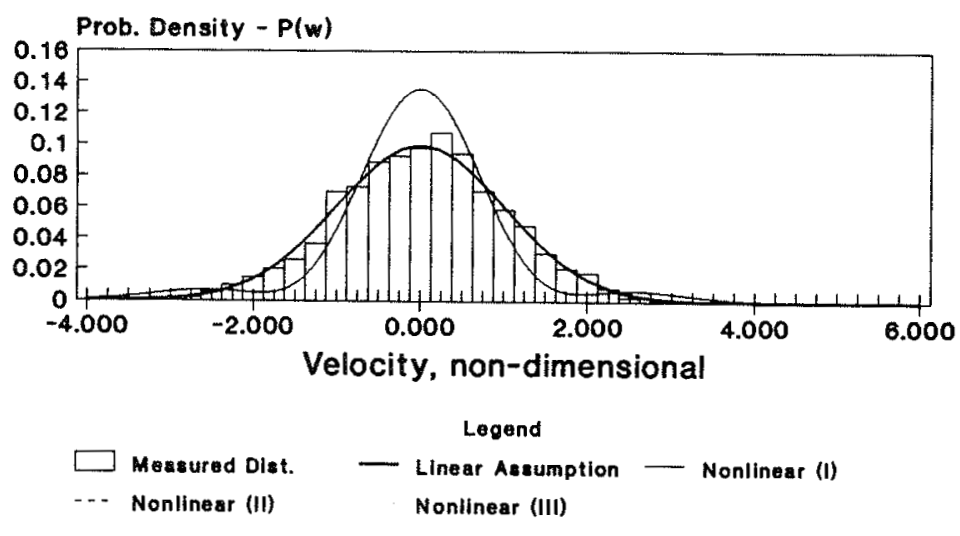

(d)

Figure 75: Time Series No. 18. (a) Filtered Data [0.20-5.00] s; (b) Filtered Data

[0.20-5.00] s; (c) Raw Data; (d) Raw Data 


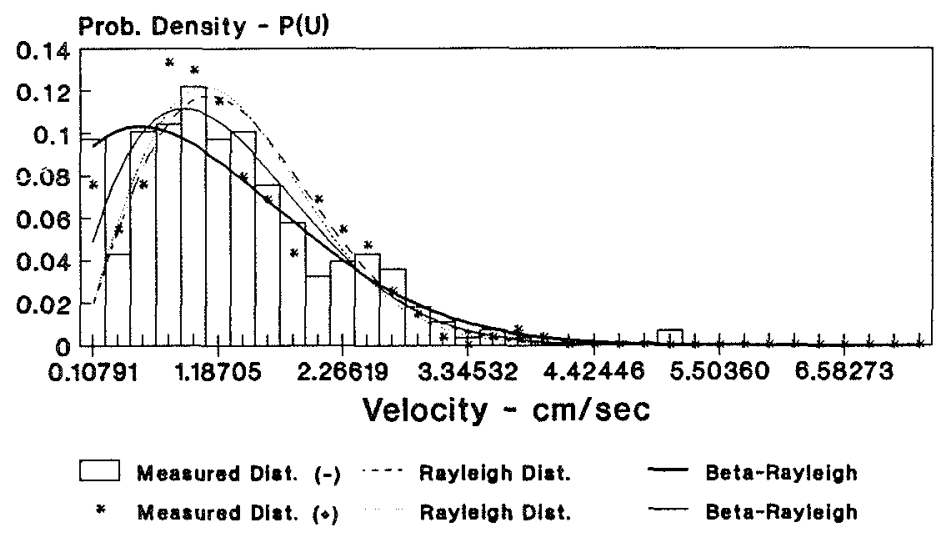

(a)

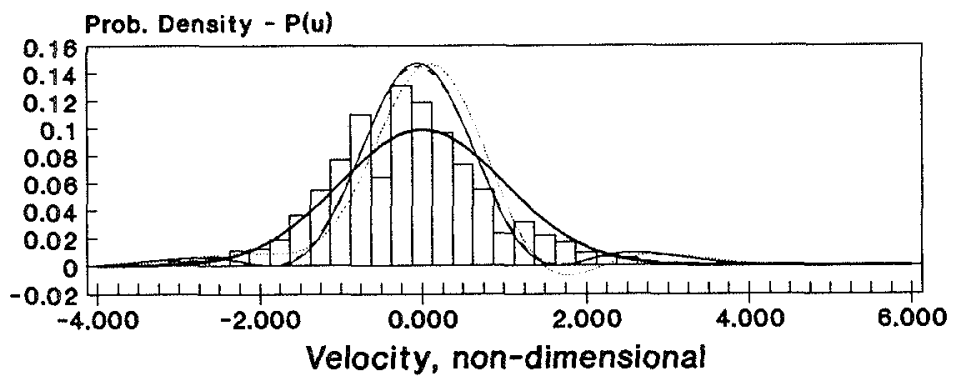

\section{Legend}

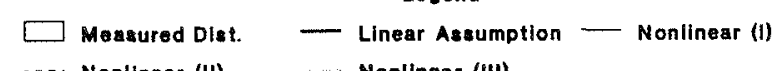

.... Nonlinear (II) .... Nonlinear (III)

(c)

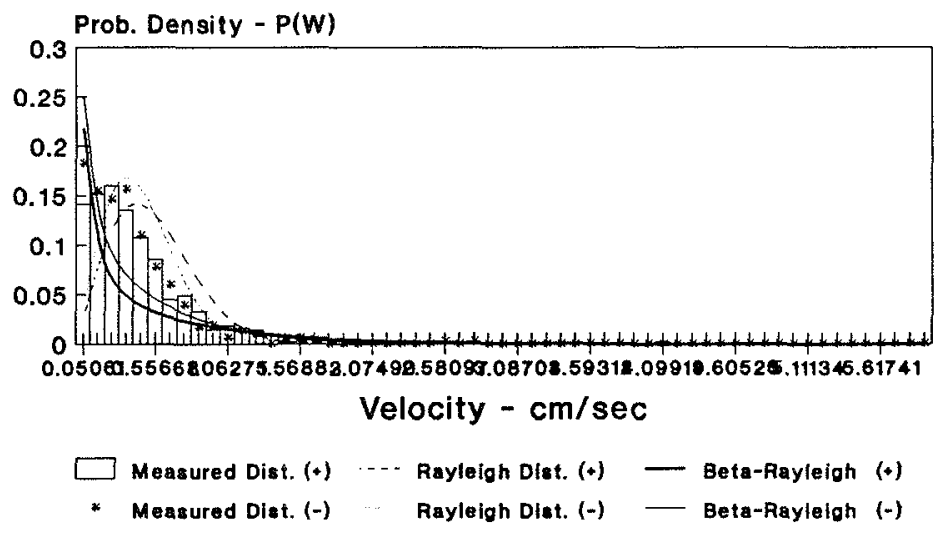

(b)

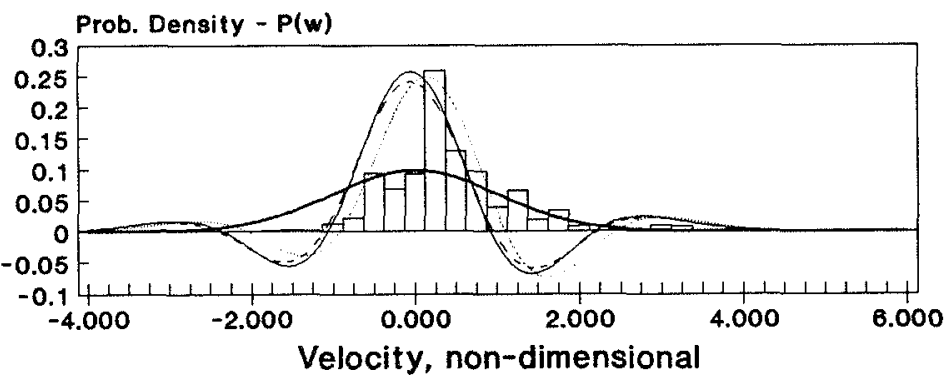

\section{Legend}

$\square$ Measured Dist. - Linear Assumption - Nonlinear (I)

(d)

Figure 76: Time Series No. 19. (a) Filtered Data [0.20-5.00] s; (b) Filtered Data

[0.20-5.00] s; (c) Raw Data; (d) Raw Data 


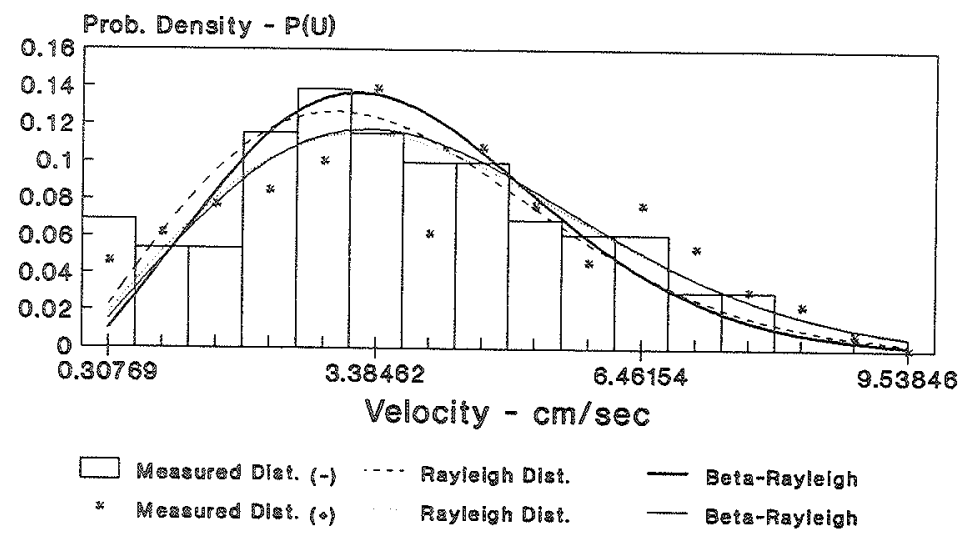

(a)
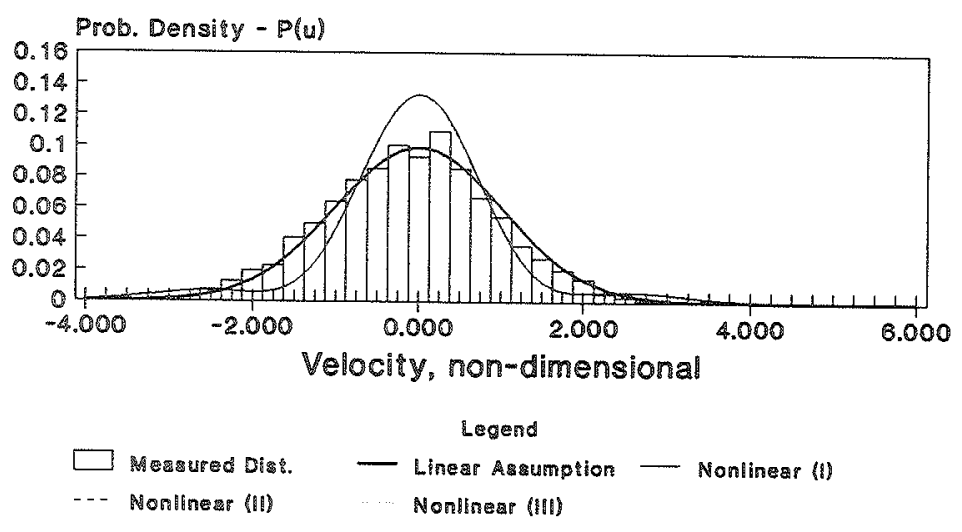

(c)

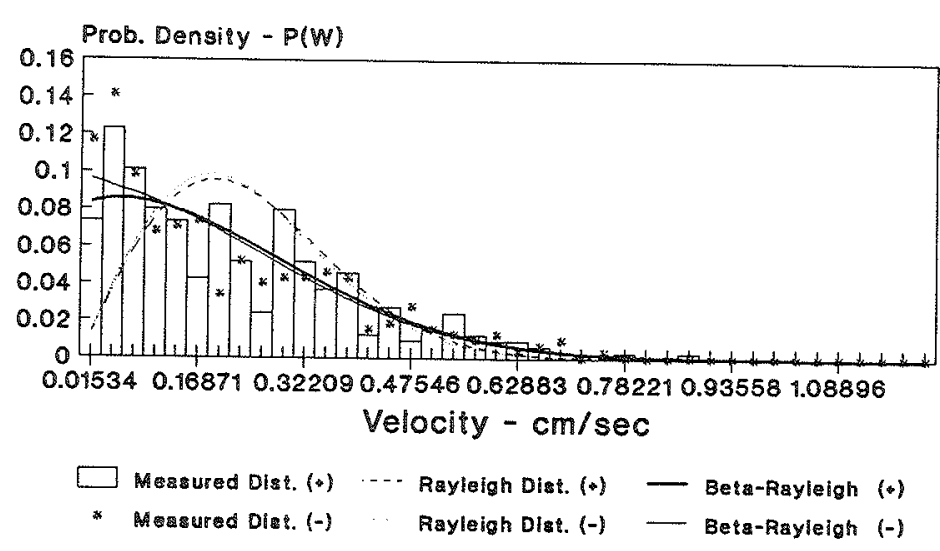

(b)

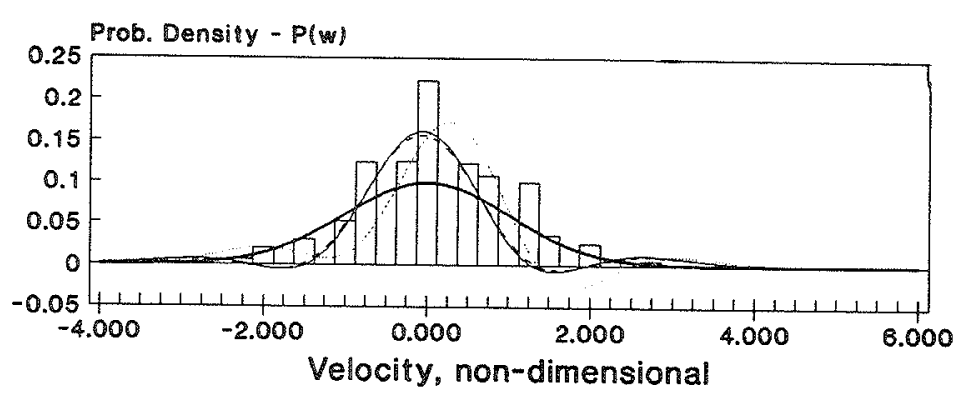

(d)

Figure 77: Time Series No. 25. (a) Filtered Data [0.20-5.00] s; (b) Filtered Data

[0.20-5.00] s; (c) Raw Data; (d) Raw Data 


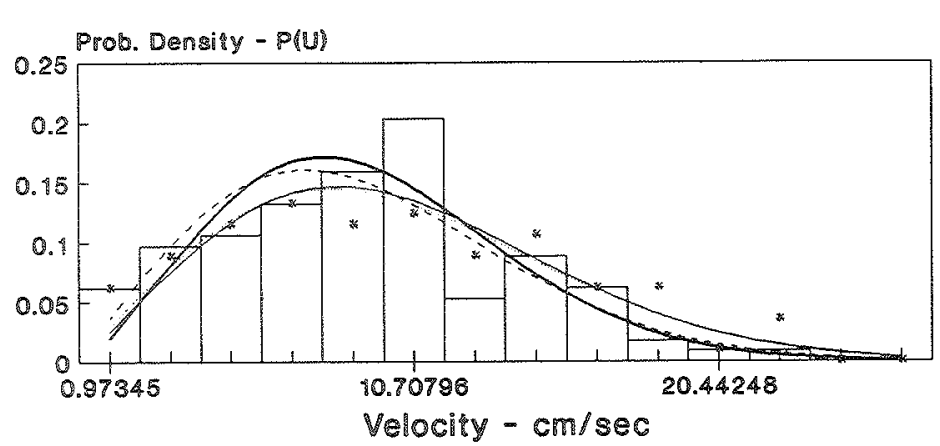

$\square$ Messured Dies. (-) .... Rayleigh Diert. - - Bata-Rayleigh - Masursd Dist. (-) …. Rayleigh Dist. - Bata-Rayleigh

(a)

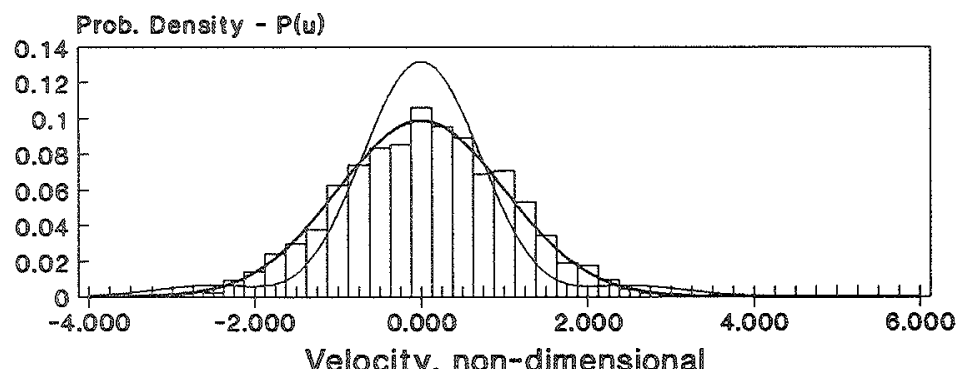

\section{Legend}

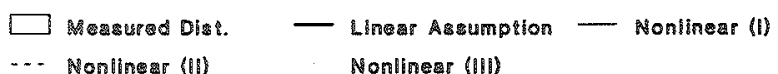

(c)

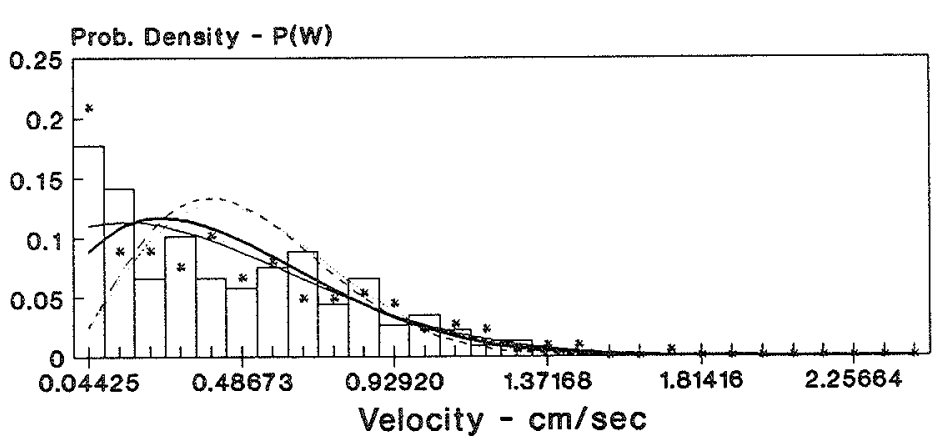

$\square$ Measured Dist. (4) -... Rayleigh Dist. (4) - Beta-Rayleigh (4)

- Memsured Dis8. (-) Rayleigh Diat. (-) - Beta-Rayleigh (-)

(b)

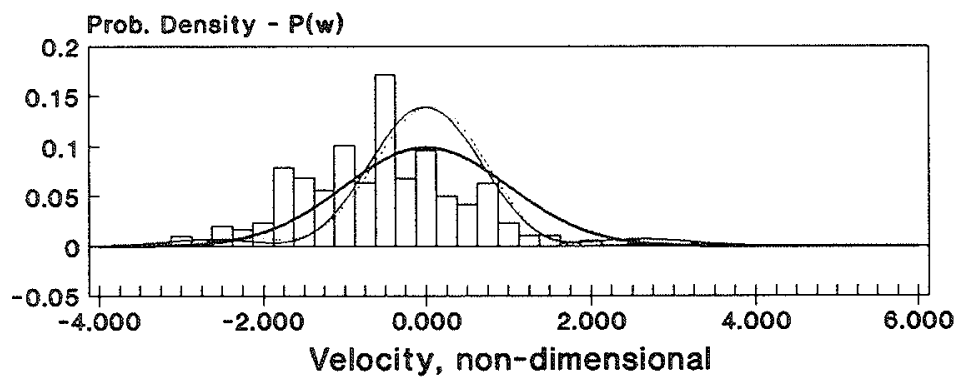

Legend

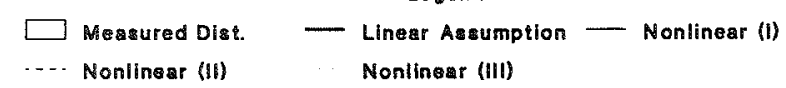

(d)

Figure 78: Time Series No. 28. (a) Filtered Data [0.20-5.00] s; (b) Filtered Data

[0.20-5.00] s; (c) Raw Data; (d) Raw Data 


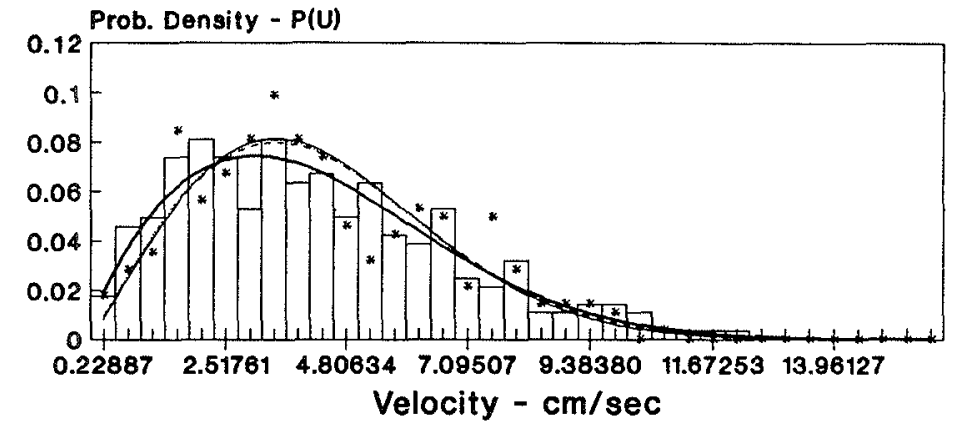

$\square$ Messured Dist. (-) … Rayloigh Dist. - Beta-Rayleigh * Masurod Dist. (1) -... Raylelgh Dlat. - Beta-Rayleigh

(a)

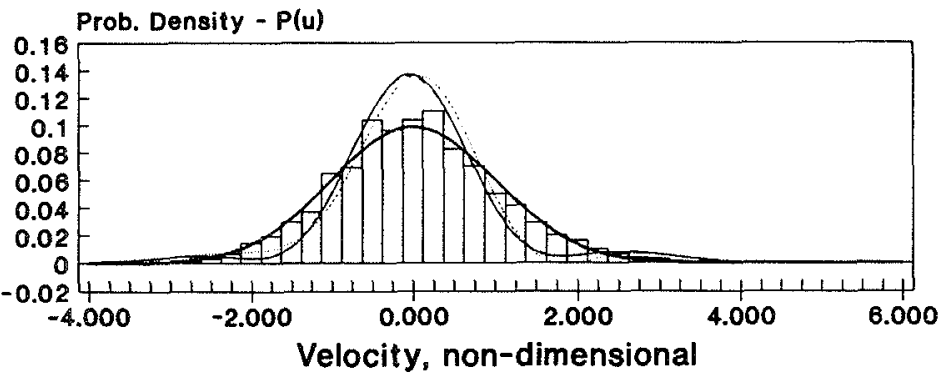

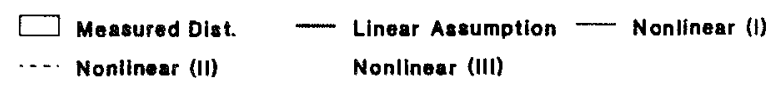

(c)

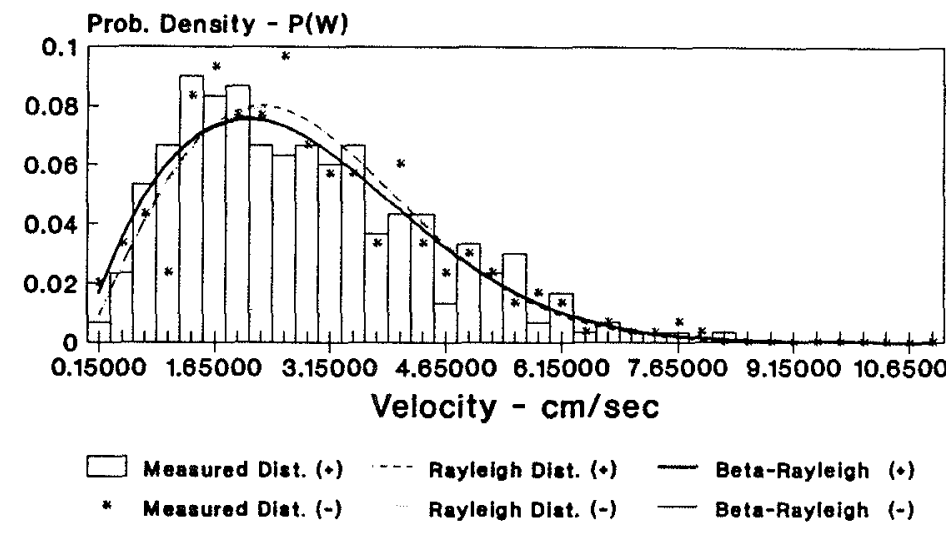

(b)

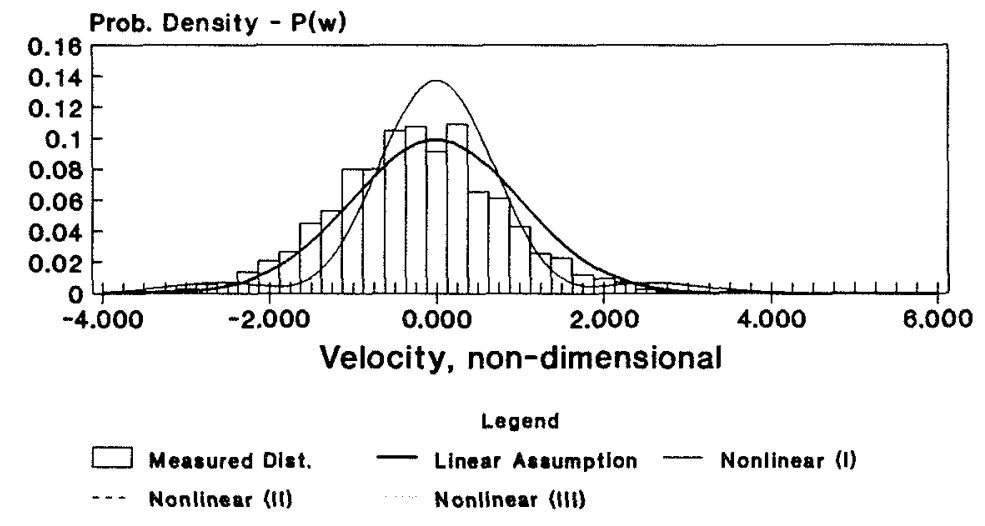

(d)

Figure 79: Time Series No. 29. (a) Filtered Data [0.20-5.00] s; (b) Filtered Data

[0.20-5.00] s; (c) Raw Data; (d) Raw Data 


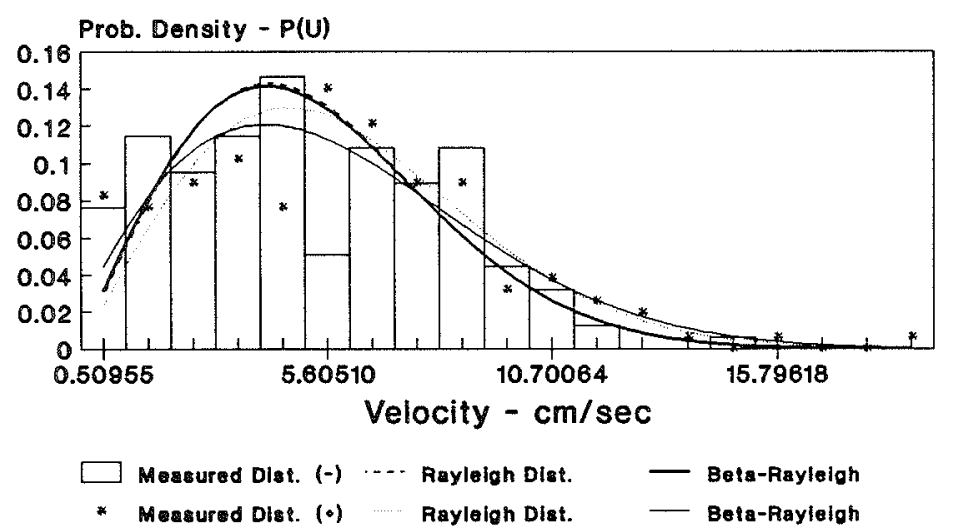

(a)
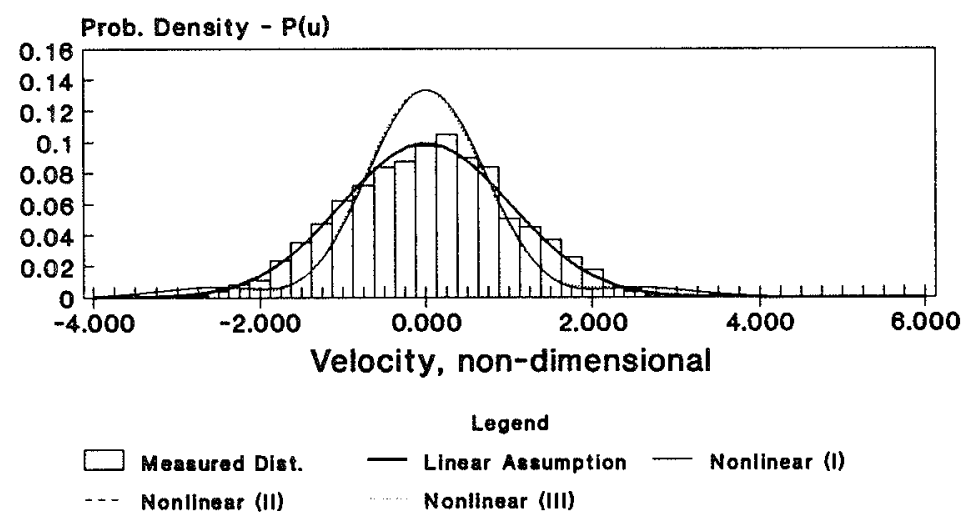

(c)

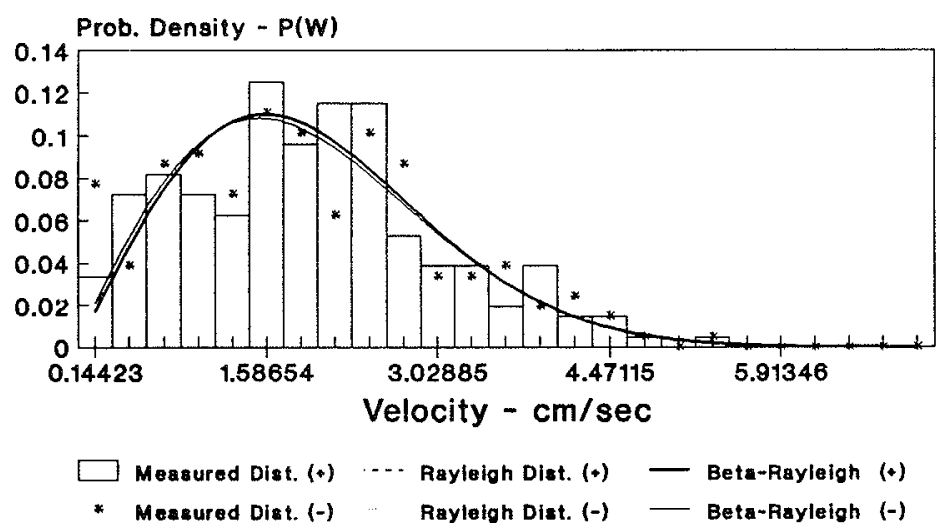

(b)

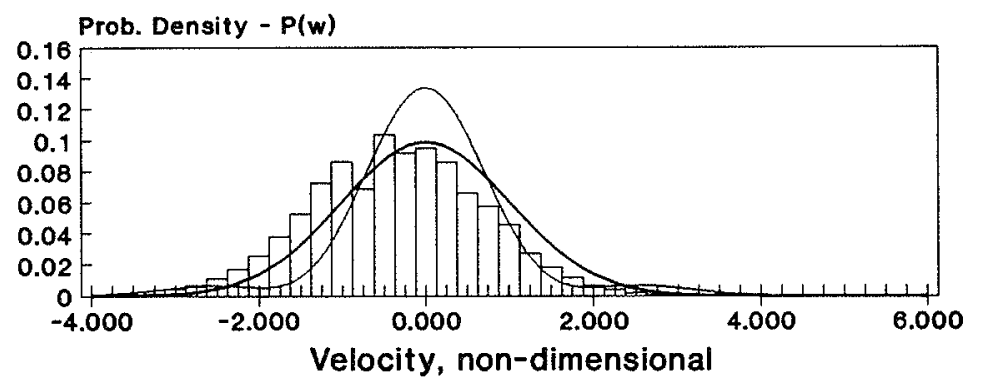

$$
\begin{aligned}
& \square \text { Measured Dist. } \quad \text { Legend } \\
& \text {-.- Nonlinear (III) Nonlinear (III) }
\end{aligned}
$$

(d)

Figure 80: Time Series No. 35. (a) Filtered Data [0.20-5.00] s; (b) Filtered Data

[0.20-5.00] s; (c) Raw Data; (d) Raw Data 


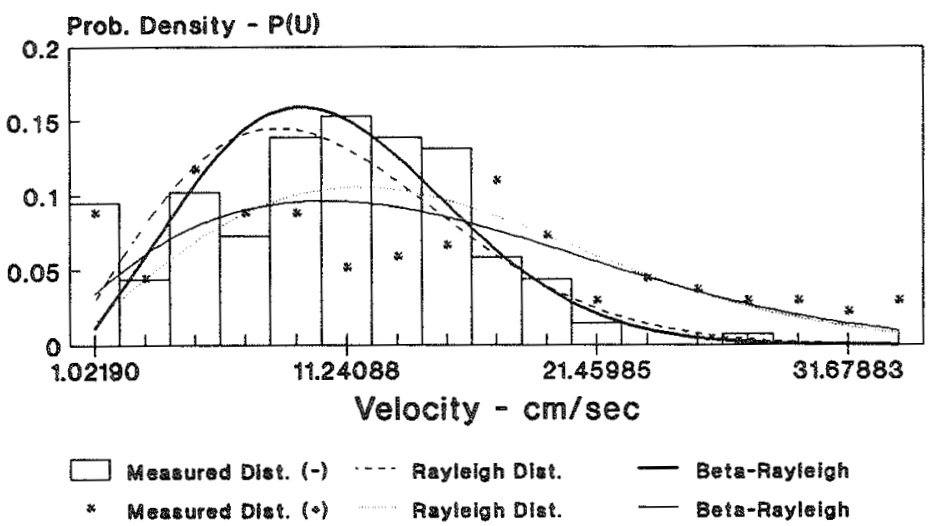

(a)

$\stackrel{\leftarrow}{\infty}$

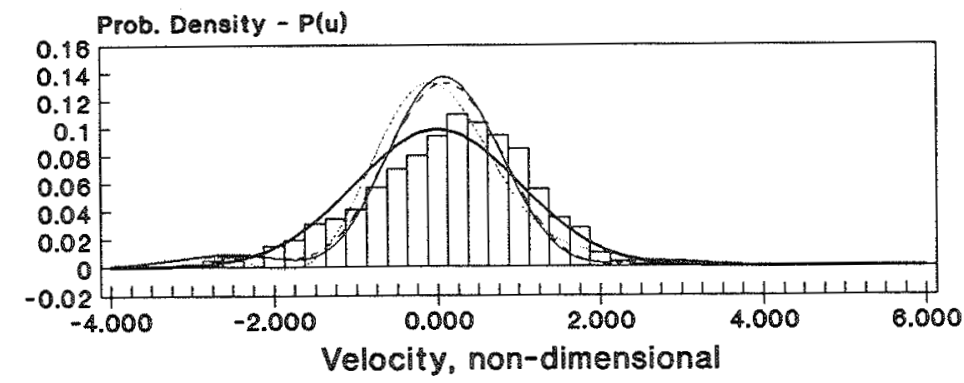

$$
\text { Legend }
$$

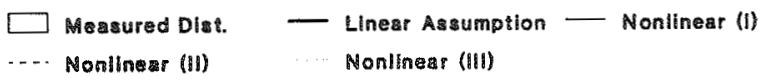

(c)

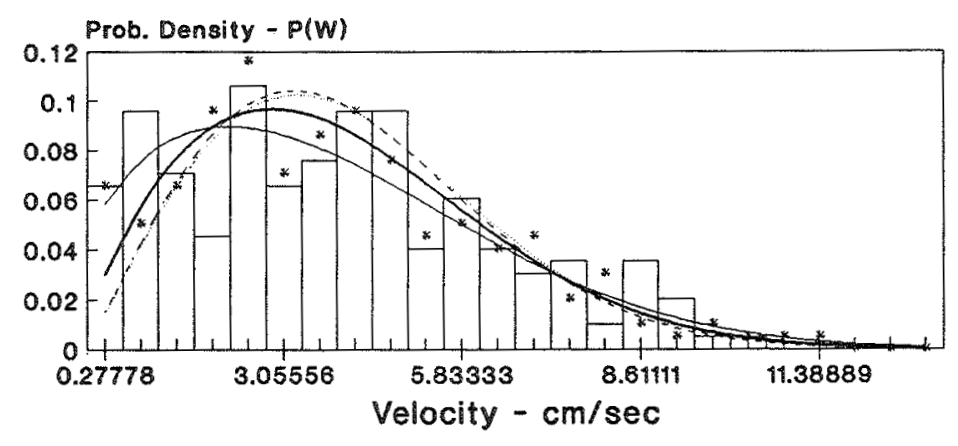

$\square$ Measured Dist. (*) -.. Rayleigh Dist. (*) — Beta-Rayleigh (*)

* Masured Dist. (-) Rayleigh Diat. (-) - Beta-Rayleigh (-)

(b)

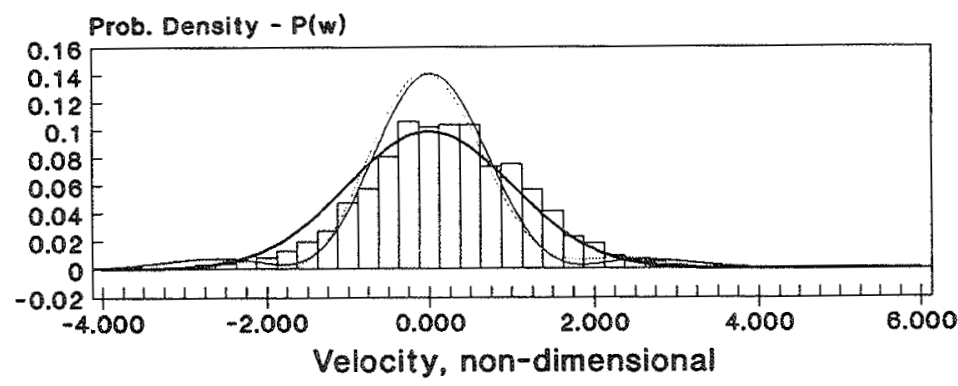

$$
\begin{aligned}
& \square \text { Measured Dizt. - Linear Aseumption - Nonlinear (1) } \\
& \text {-.- Nonlinear (II) ... Nonlinear (III) }
\end{aligned}
$$

Figure 81: Time Series No. 38. (a) Filtered Data [0.20-5.00] s; (b) Filtered Data [0.20-5.00] s; (c) Raw Data; (d) Raw Data 


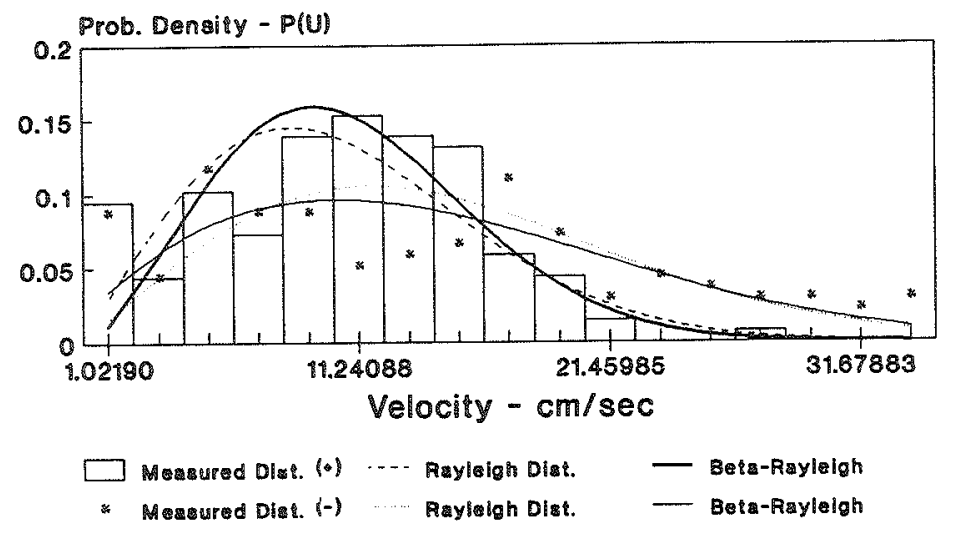

(a)
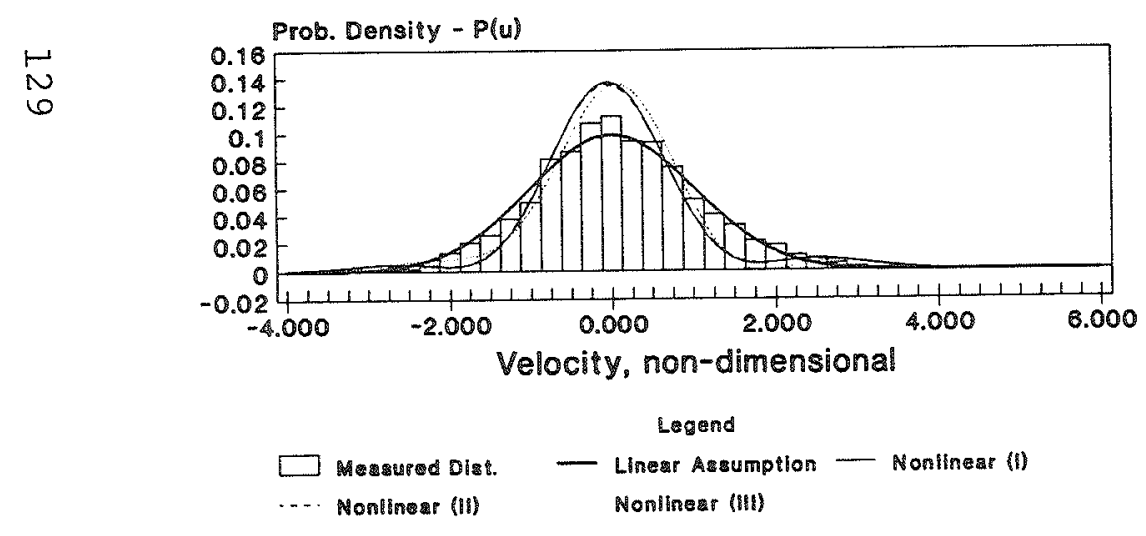

(d)

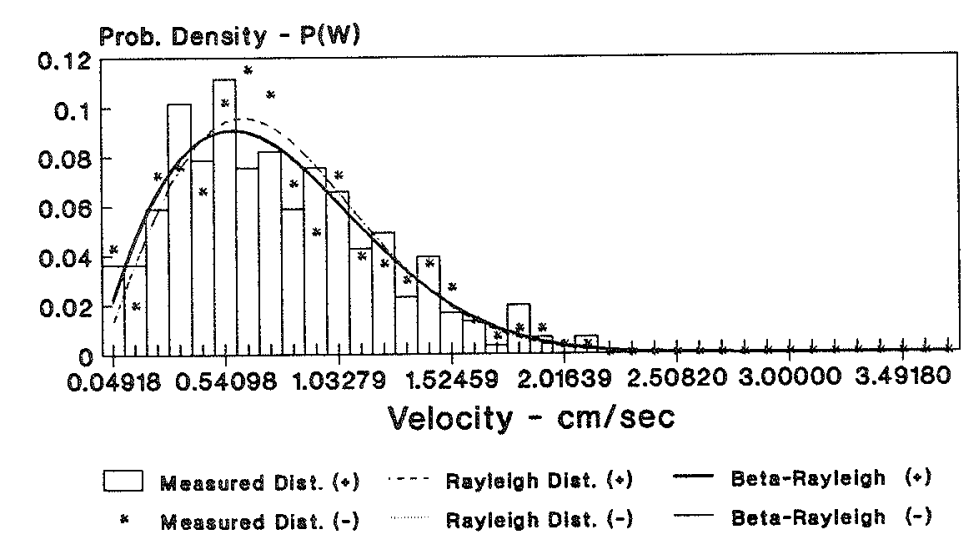

(b)
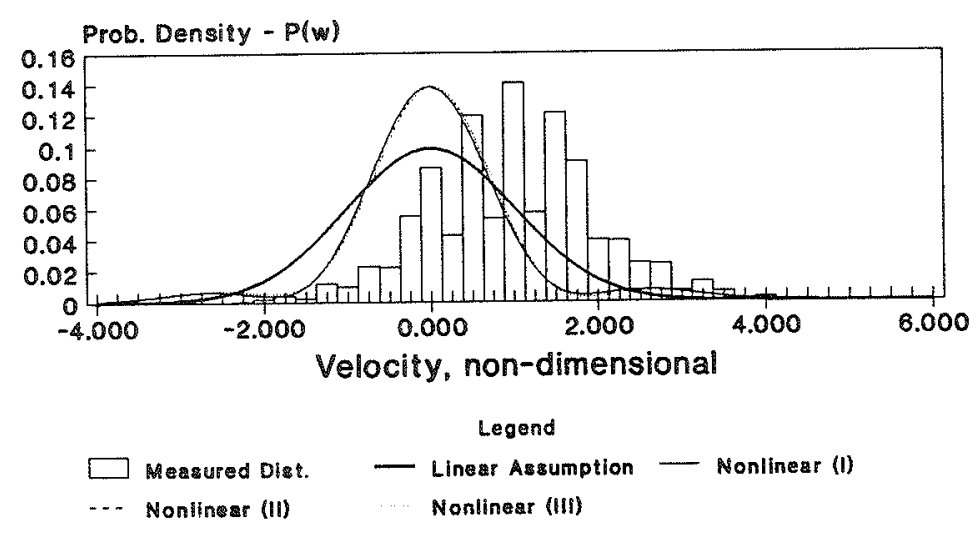

(c)

Figure 82: Time Series No. 39. (a) Filtered Data [0.20-5.00] s; (b) Filtered Data

[0.20-5.00] s; (c) Raw Data; (d) Raw Data 


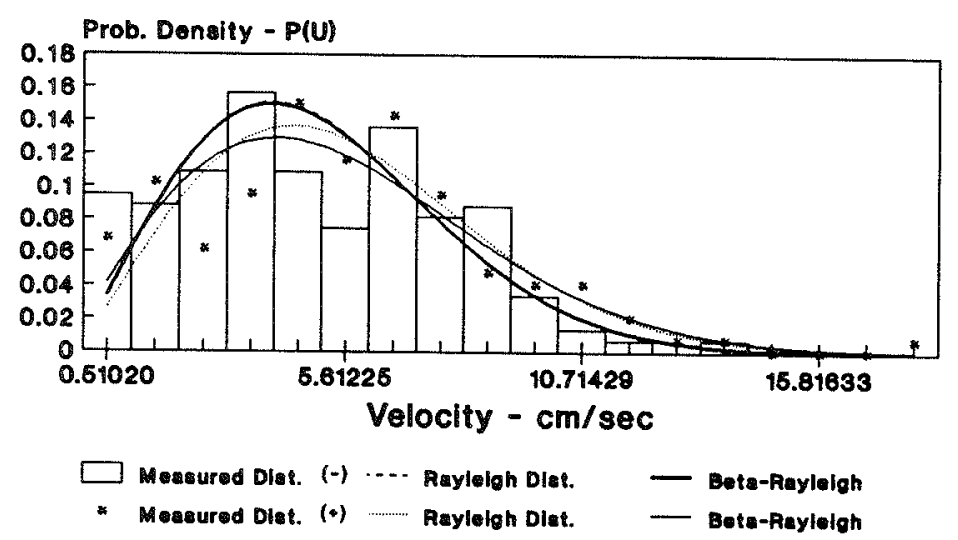

(a)

$\ddot{\omega}$

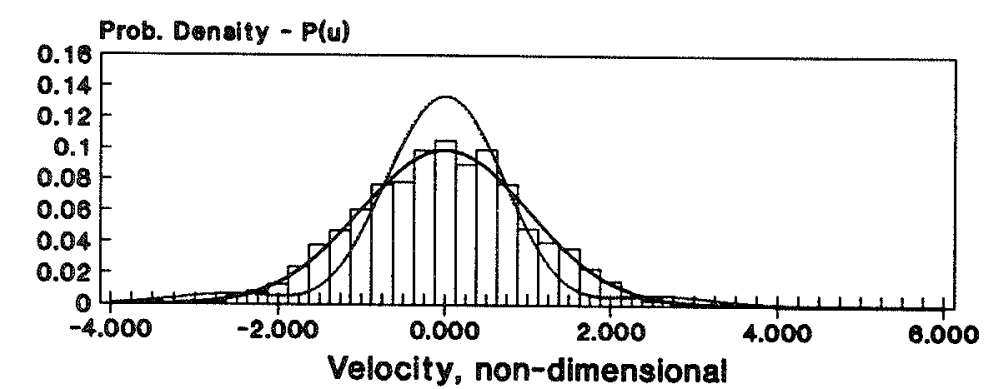

Legend

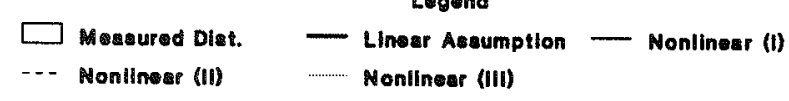

(d)

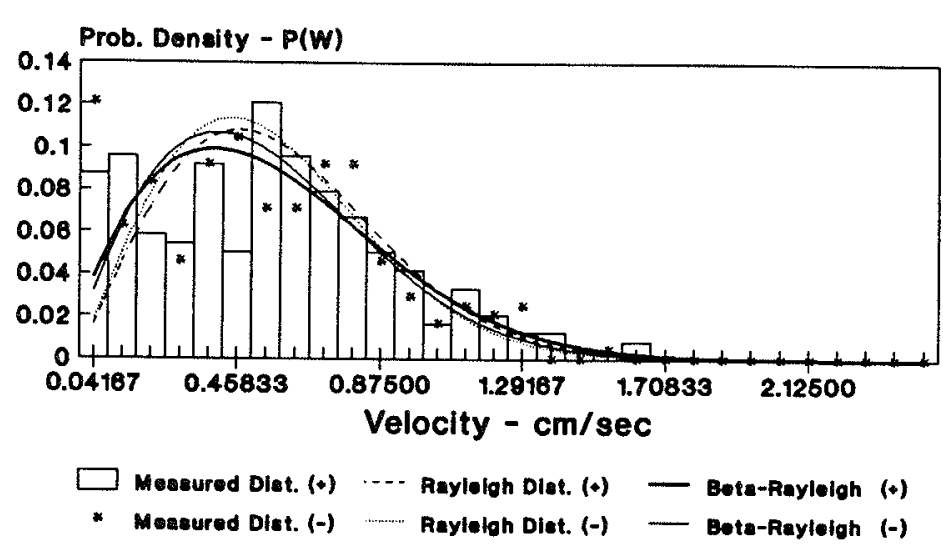

(b)

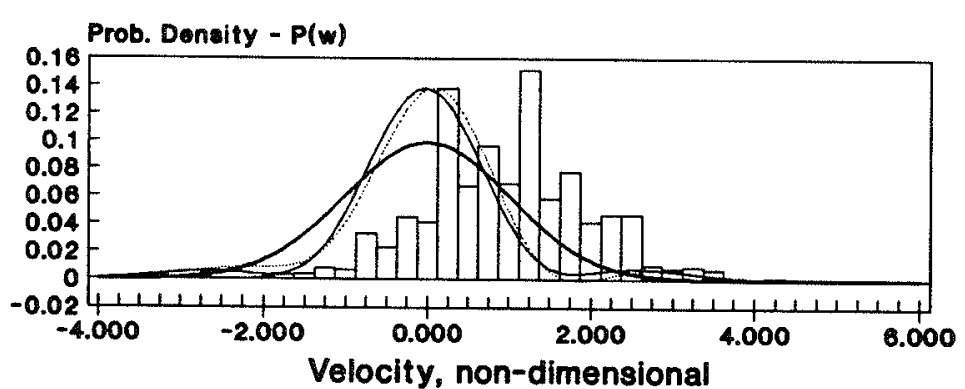

Legend

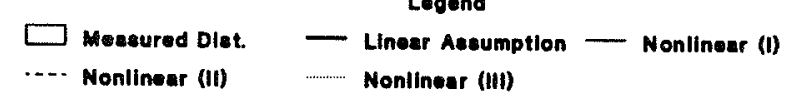

(c)

Figure 83: Time Series No. 45. (a) Filtered Data [0.20-5.00] s; (b) Filtered Data

[0.20-5.00] s; (c) Raw Data; (d) Raw Data 


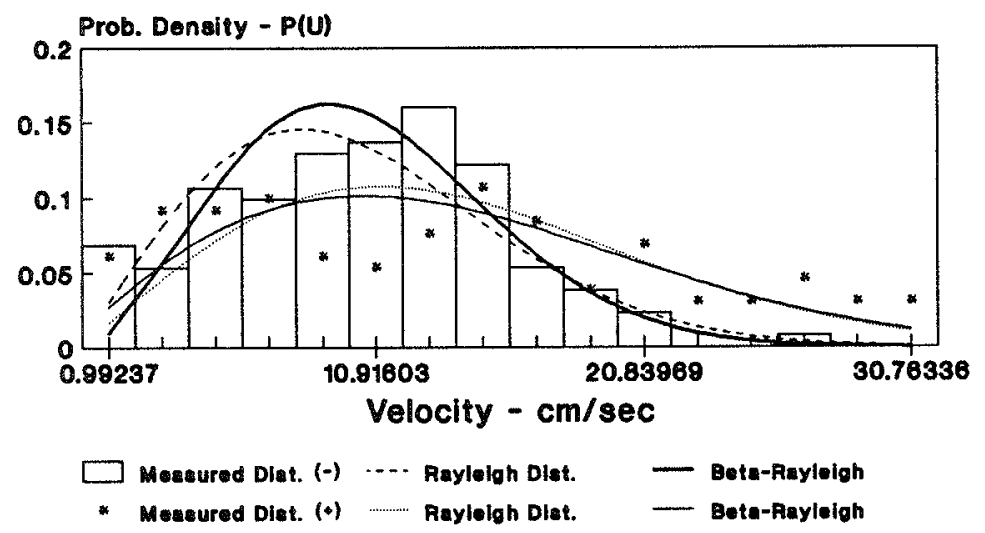

(a)

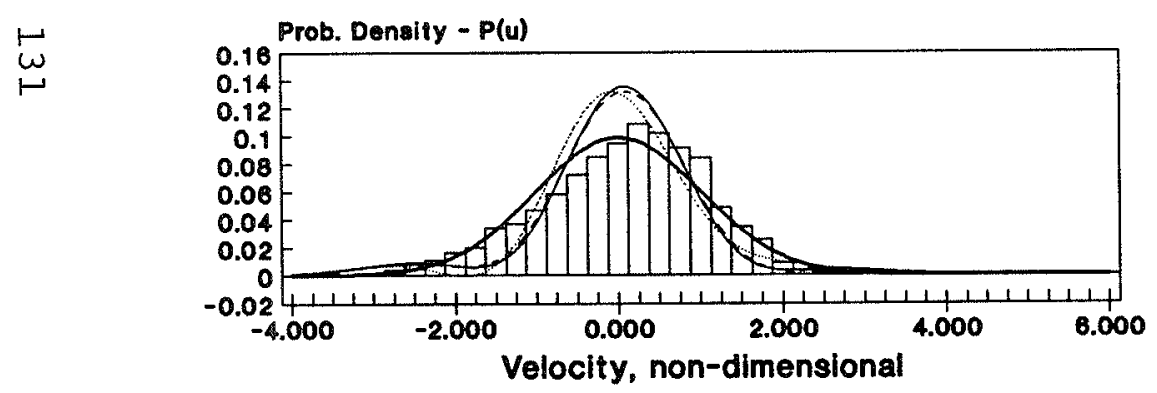

\section{Logend}

$\underset{\text { Measured Dlet. }}{\square \text {-.. Lonlinear (II) }}$ Lonnear Aesumption - Nonllinear (I)

(c)

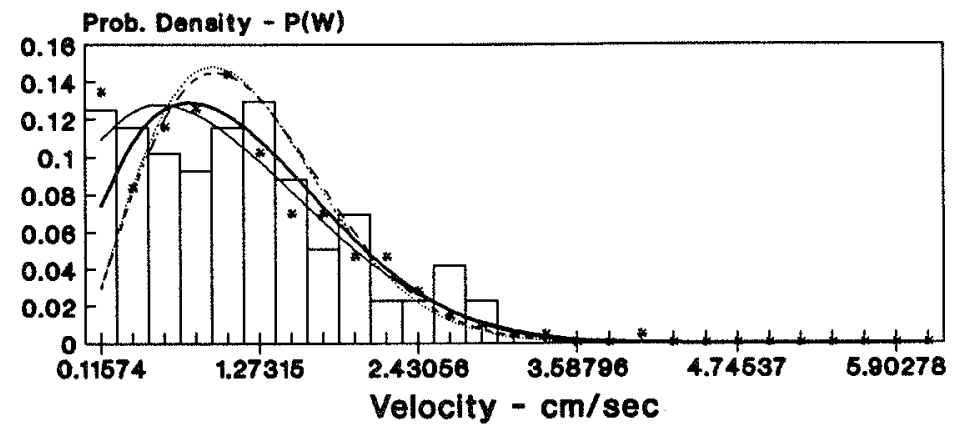

$\square$ Meseured Diet. (4) … Rayleloh Dist. (*) — Bete-Rayleigh (+)

* Measured Dist. (-) …… Rayloigh Diat. (-) — Bota-Raylolgh (-)

(b)

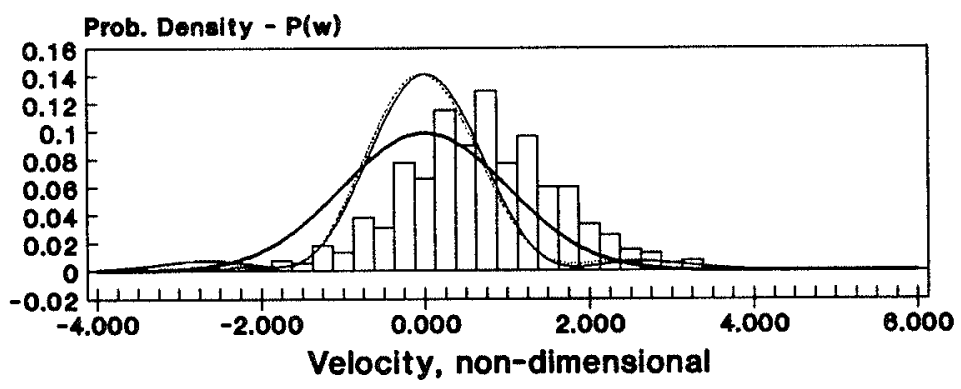

$$
\square \text { Measured Diat. } \quad \text { L Linoer Asaumption - Nonllnoar (I) }
$$

(d)

Figure 84: Time Series No. 48. (a) Filtered Data [0.20-5.00] s; (b) Filtered Data

[0.20-5.00] s; (c) Raw Data; (d) Raw Data 


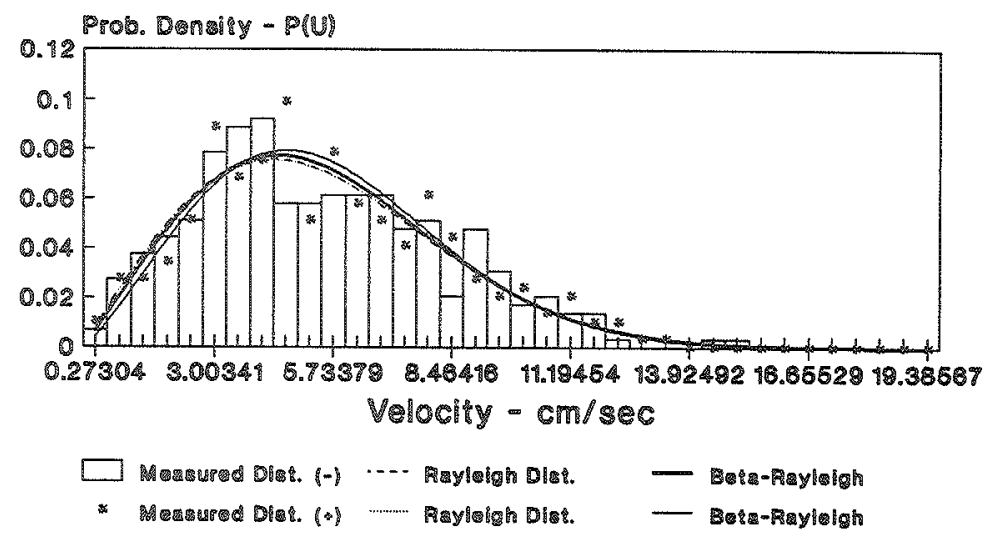

(a)

$\stackrel{\leftrightarrow}{\omega}$

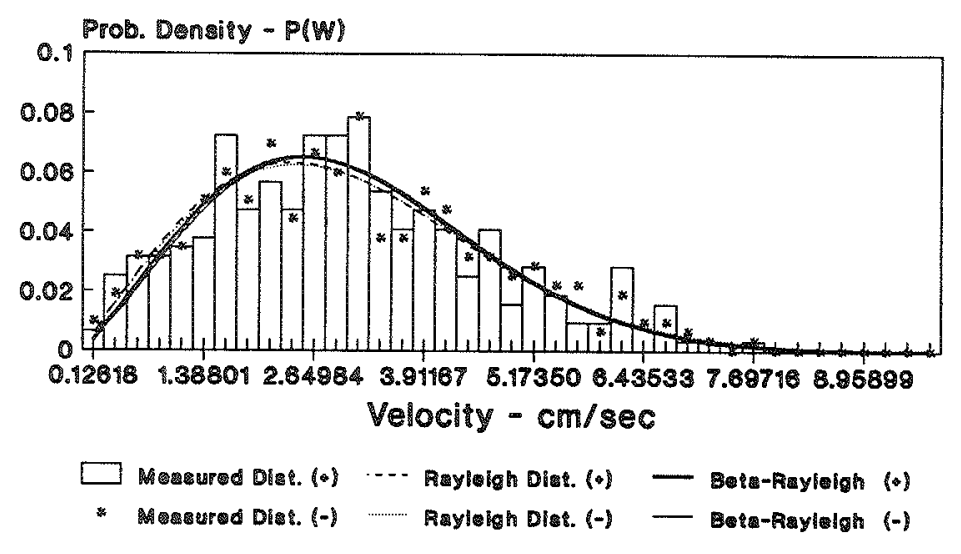

(b)
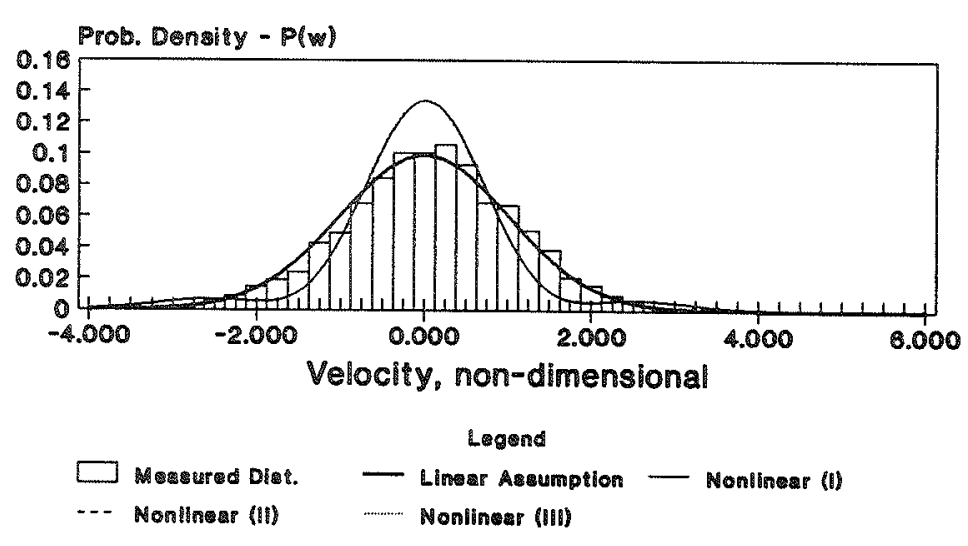

(d)

Figure 85: Time Series No. 49. (a) Filtered Data [0.20-5.00] s; (b) Filtered Data

[0.20-5.00] s; (c) Raw Data; (d) Raw Data 


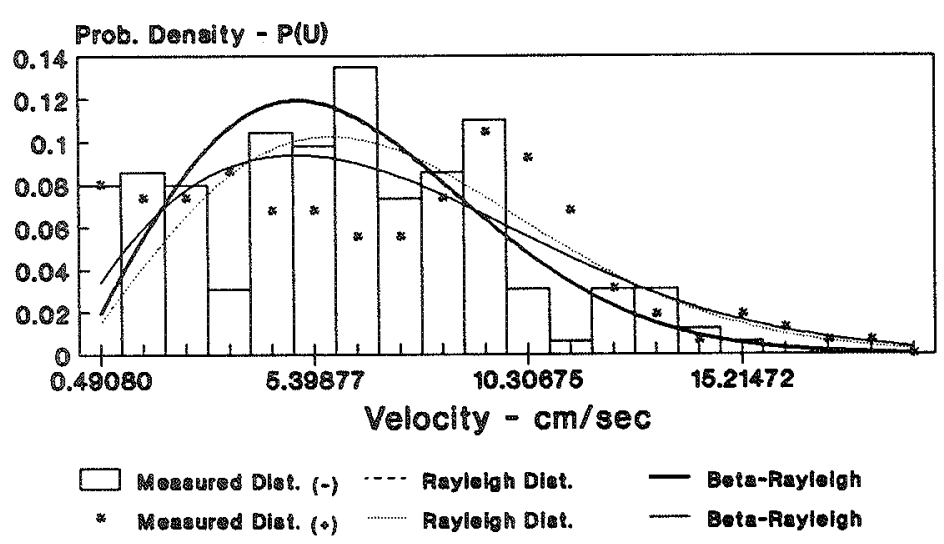

(a)

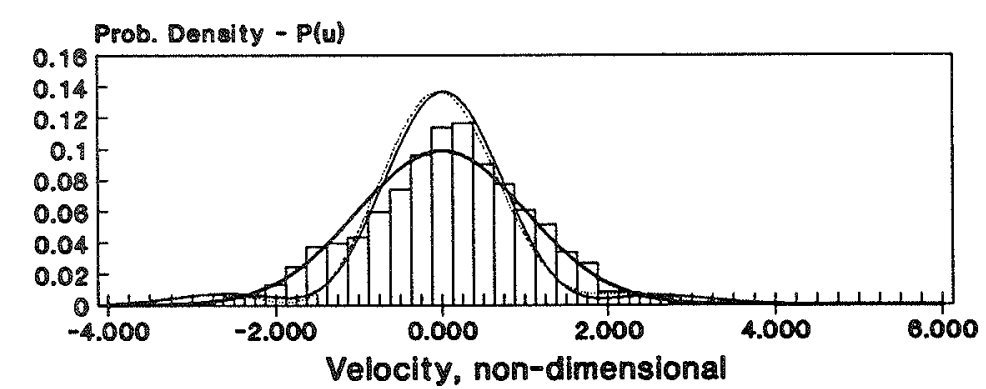

\section{Legend}

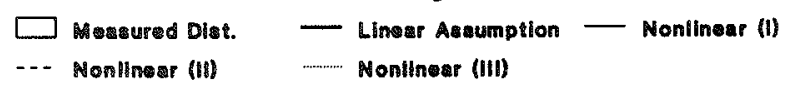

(c)

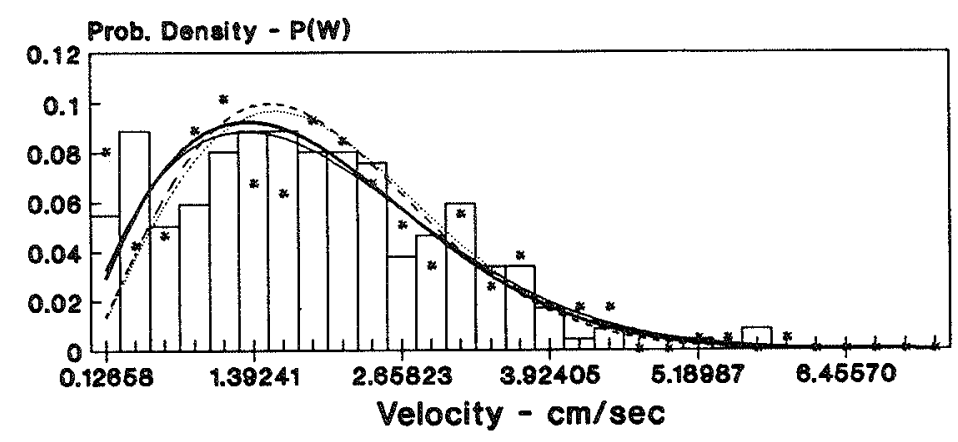

$\square$ Moanured Diat. (1) -..- Raylaigh Diat. (0) — Beta-Rayleloh (+)

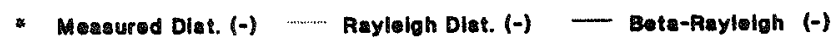

(b)
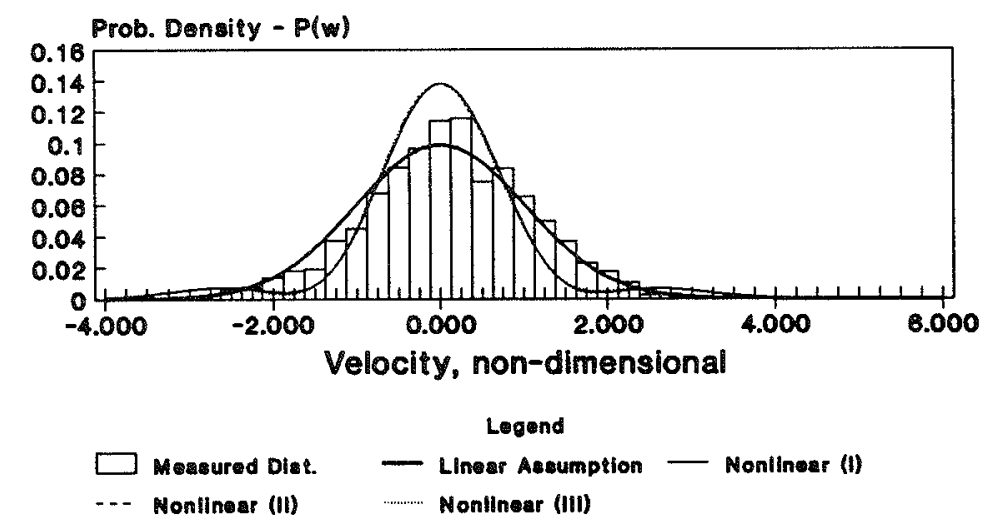

(d)

Figure 86: Time Series No. 55. (a) Filtered Data [0.20-5.00] s; (b) Filtered Data [0.20-5.00] s; (c) Raw Data; (d) Raw Data 


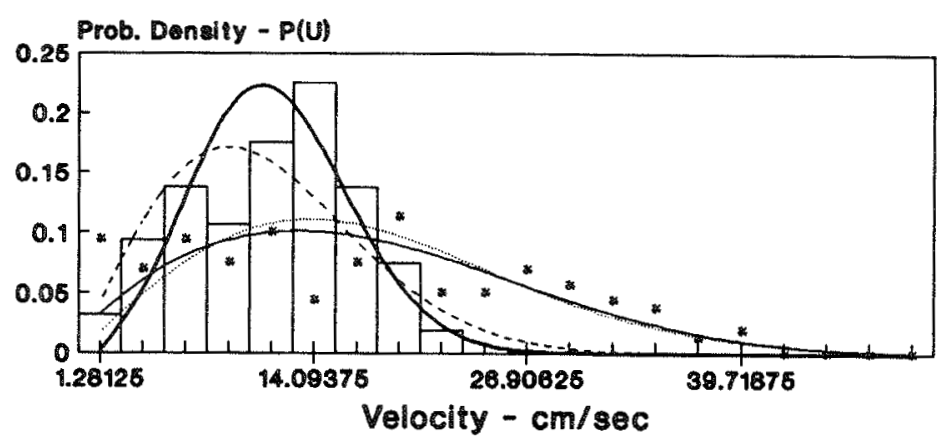

$\square$ Masaursed Dlet. (-) … Raylolgh Dlat. - Buta-Rayleigh

" Moscured Diet. (4) Rayleigh Dlet. - Bota-Raylolgh

(a)

$\stackrel{\omega}{\omega}$

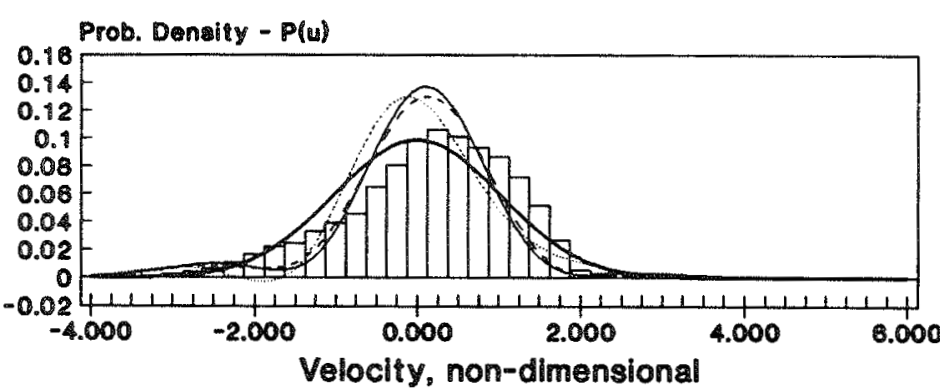

\section{Logond}

$\square$ Measured Diet. - Linsar Azeumption - Nonllnear (1)

-... Nonlinear (II) -........ Nonlinear (III)

(c)
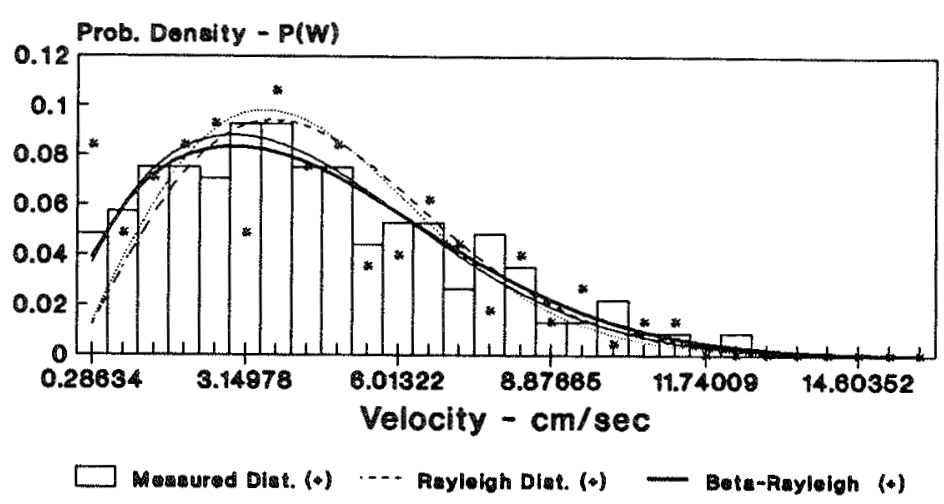

* Moneured Dlet. (-) Rayleigh Dist. (-) - Bota-Raylelgh (-)

(b)

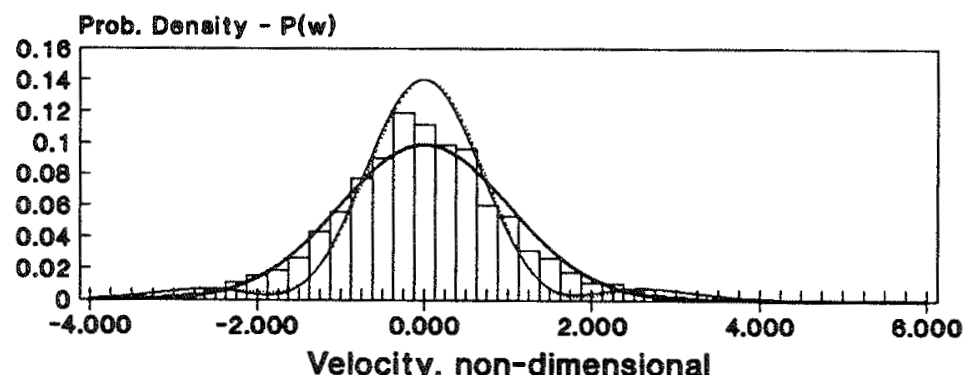

Legend

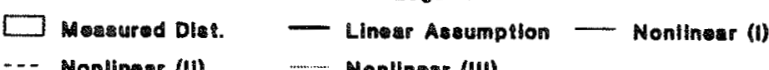

-.- Nonlinear (II) -...- Nonlinear (III)

(d)

Figure 87: Time Series No. 58. (a) Filtered Data [0.20-5.00] s; (b) Filtered Data

[0.20-5.00] s; (c) Raw Data; (d) Raw Data 


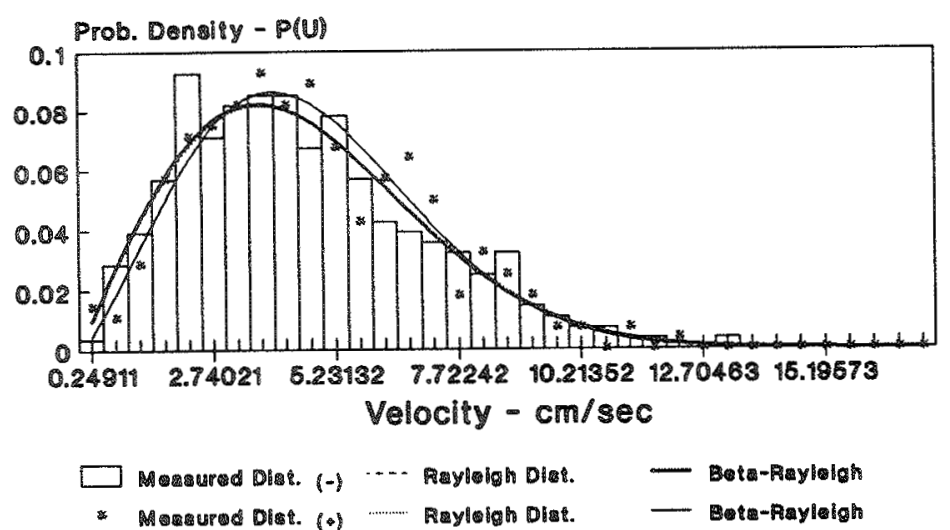

(a)

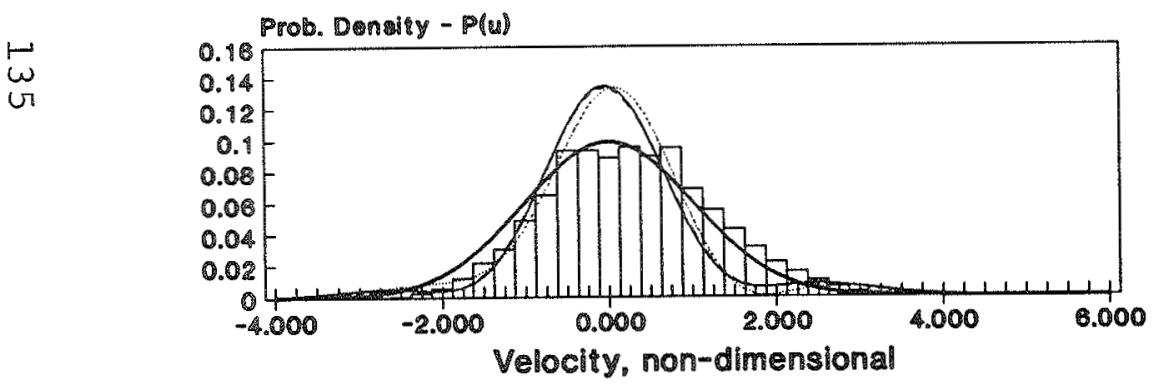

$$
\begin{aligned}
& \text { Legend } \\
& \text { D.- Nonlinear (III) }
\end{aligned}
$$

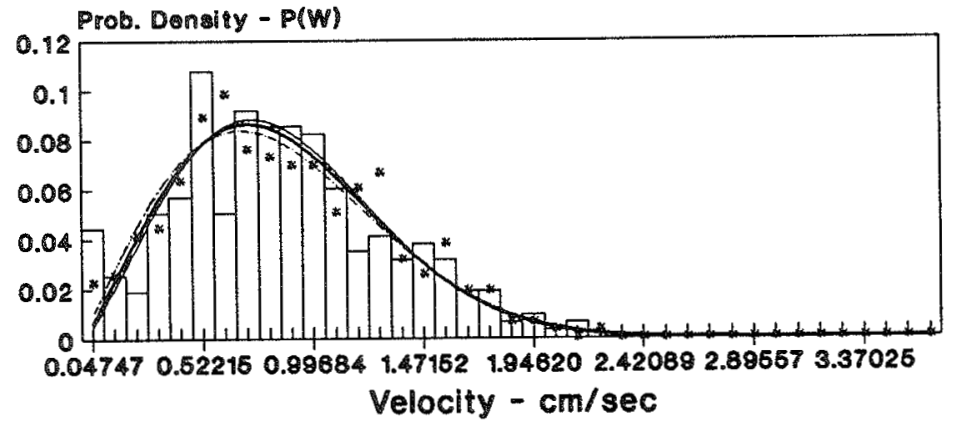

$\square$ Maeured Dist. (+) -..- Rayleigh Dist. (o) — Beta-Raylelgh (+)

* Measured Dist. (-) - - T.... Rayleigh Dlet. (-) - Beta-Rayleigh (-)

(b)

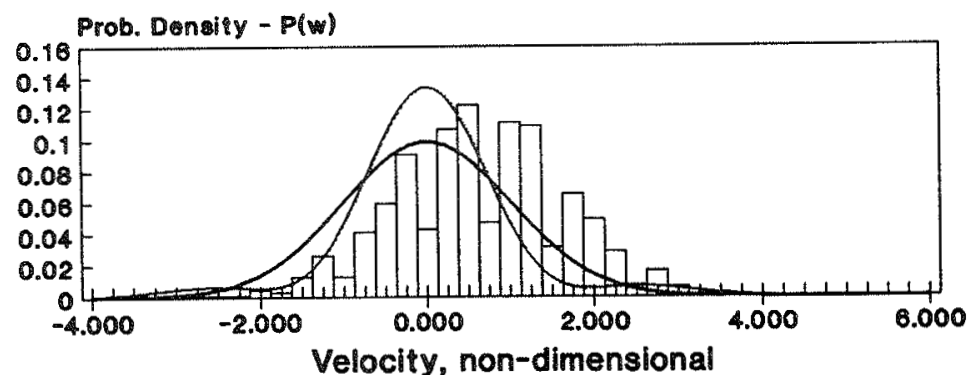

\section{Logend}

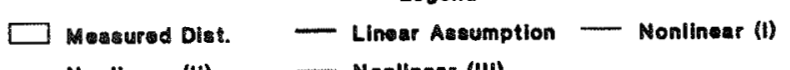

-.. Nonllnear (II) -..... Nonlinear (III)

(d)

Figure 88: Time Series No. 59. (a) Filtered Data [0.20-5.00] s; (b) Filtered Data [0.20-5.00] s; (c) Raw Data; (d) Raw Data 


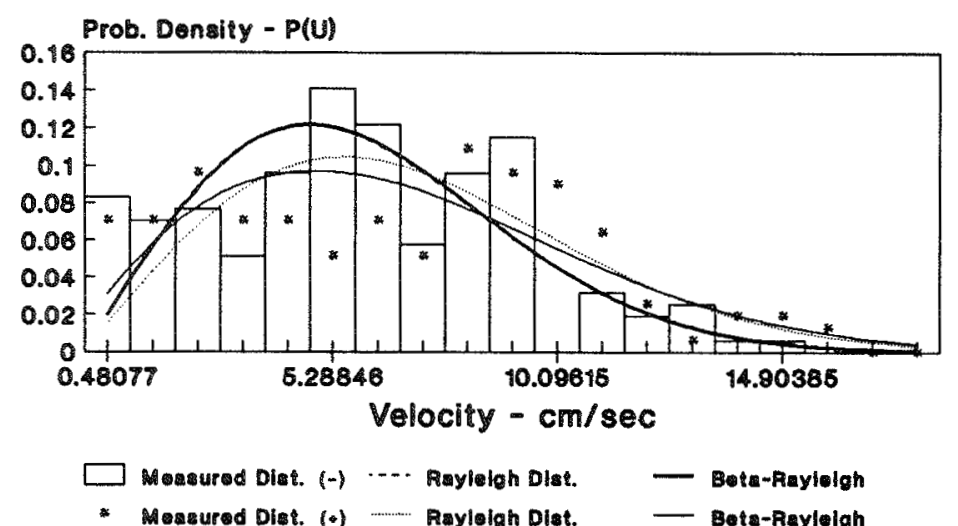

(a)

$\stackrel{\omega}{\sigma}$

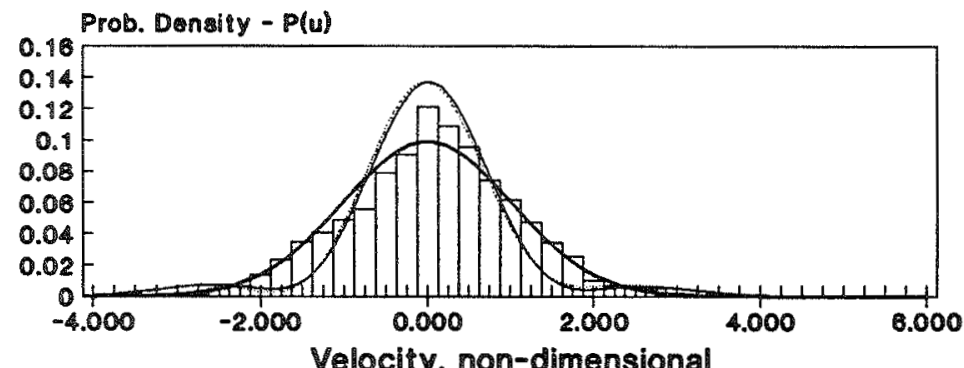

\section{L.gend}

$\square$ Meazured Dist. - Linear Assumption - Nonlinear (II)

(c)

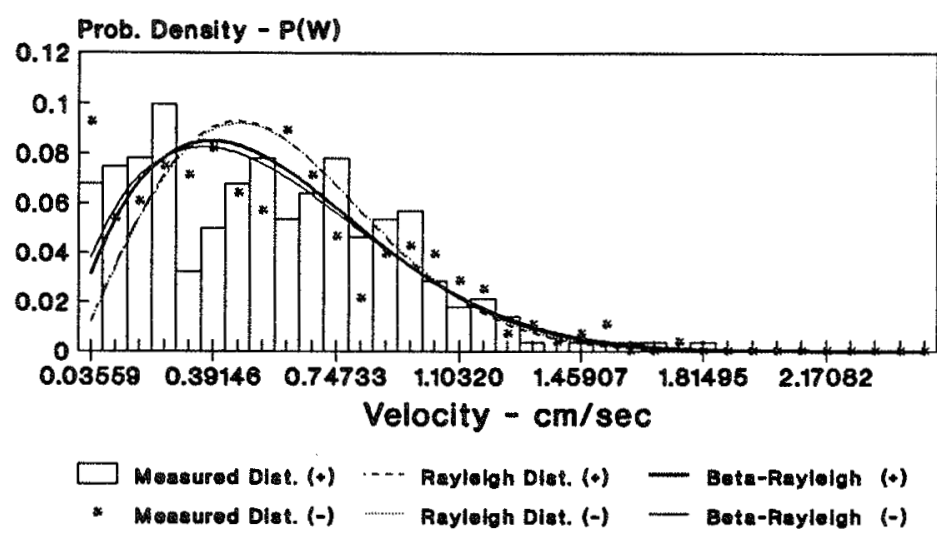

(b)

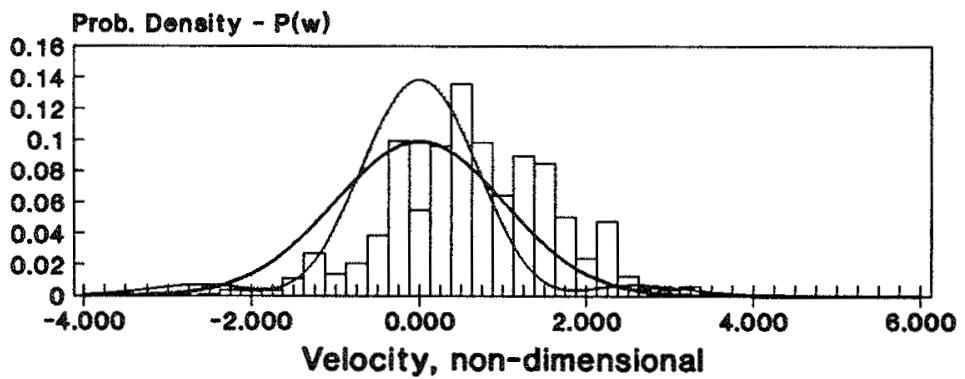

Logend

$\square$ Measured Dist. - Linesr Aseumption - Nonlinear (I)

-.. Nonllnear (II) None Nonlinear (III)

(d)

Figure 89: Time Series No. 65. (a) Filtered Data [0.20-5.00] s; (b) Filtered Data

[0.20-5.00] s; (c) Raw Data; (d) Raw Data 


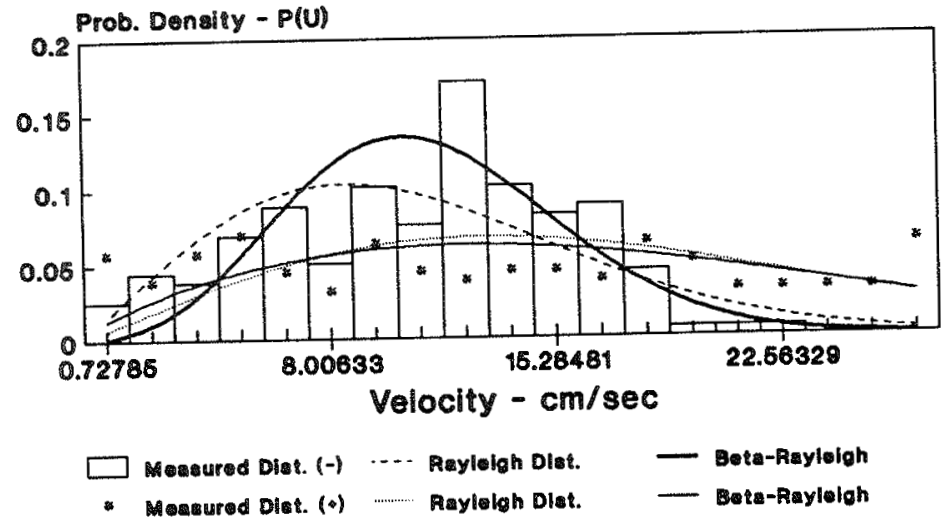

(a)

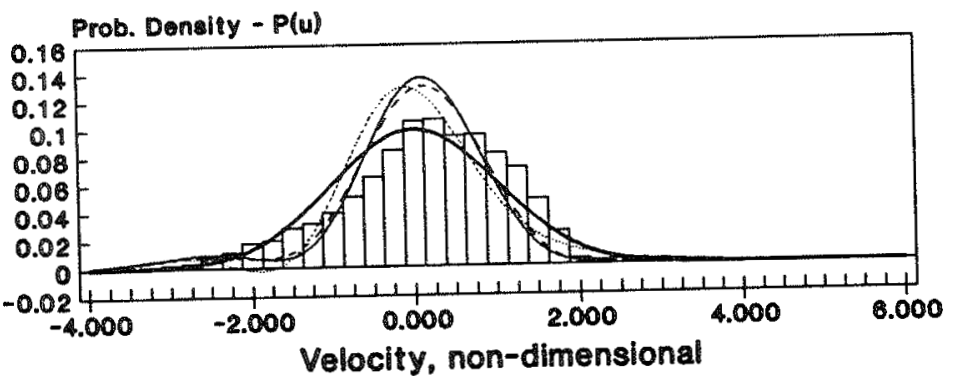

\section{Legend}

$\square$ Aneseured Dieq - Linear Aseumption - Nonlinear (1)

.... Nonlinear (II) -...... Nonlinesar (III)

(c)

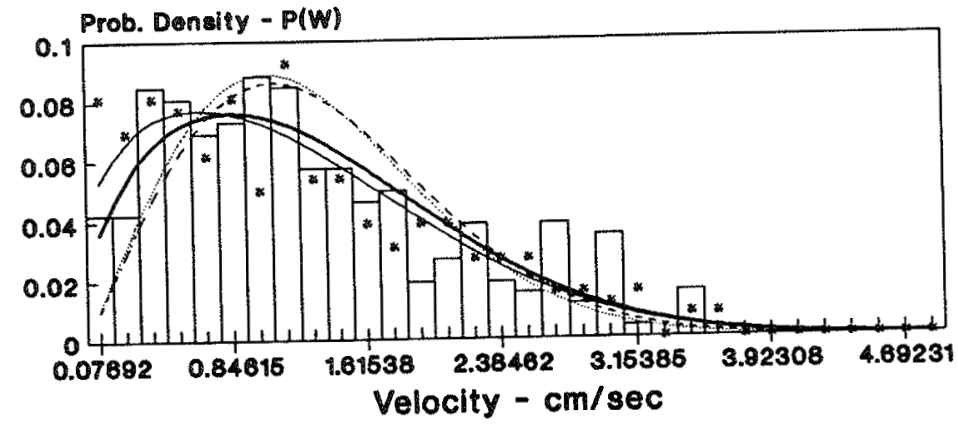

$\square$ Measured Dist. (+) … Rayleloh Diat. (+) - Betu-Rayleloh (-)

- Masured Diet. (-) Mayleigh Diet. (-) - Beta-Raylolgh (-)

(b)

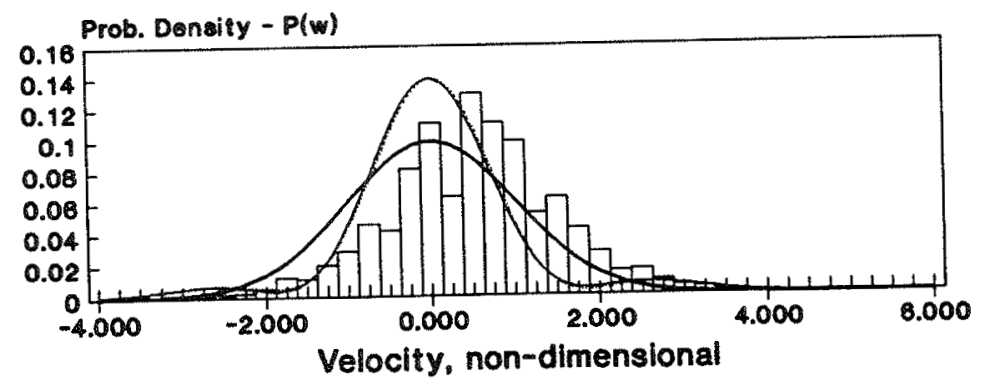

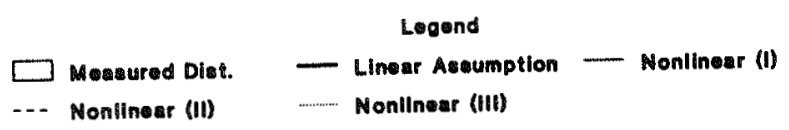

(d)

Figure 90: Time Series No. 68. (a) Filtered Data [0.20-5.00] s; (b) Filtered Data

[0.20-5.00] s; (c) Raw Data; (d) Raw Data 


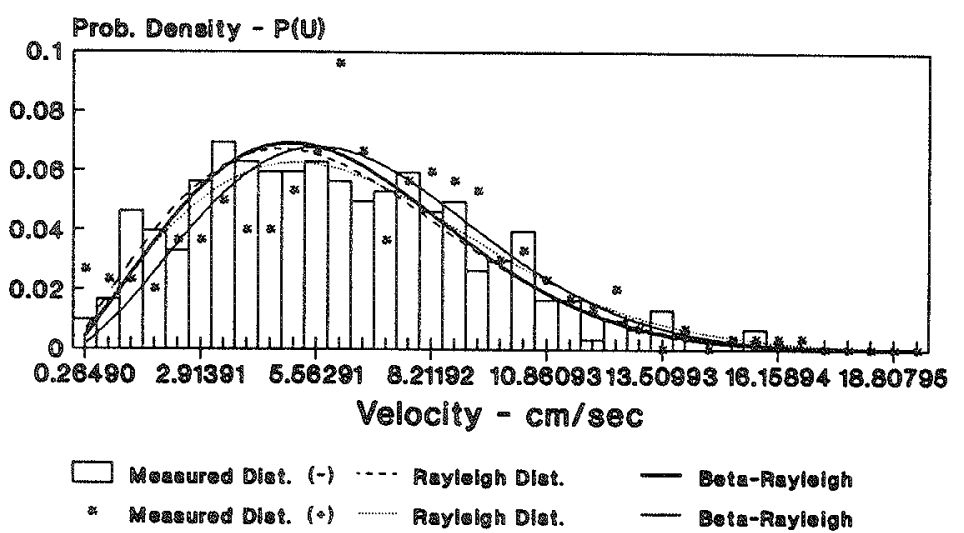

(a)

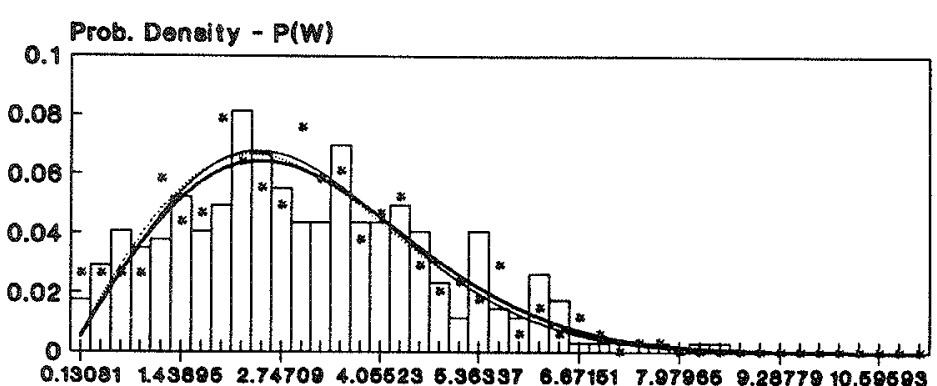
Velocity $-\mathrm{cm} / \mathrm{sec}$

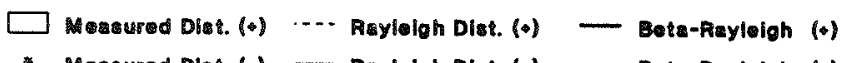

* Macured Diat. (-) -....... Raylelgh Diet. (-) - Bata-Rayleigh (-)

(b)

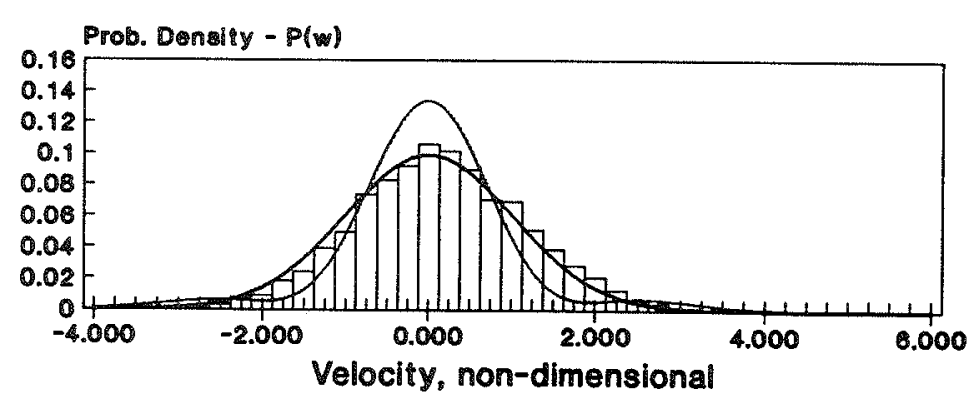

$$
\begin{aligned}
& \text { Legend } \\
& \square \text { mesesred Dist. - Linoar Aesumption - Nonlinesr (1) } \\
& \text {-.- Nonlinear (II) -......- Nonlinear (III) }
\end{aligned}
$$

(d)

Figure 91: Time Series No. 69. (a) Filtered Data [0.20-5.00] s; (b) Filtered Data [0.20-5.00] s; (c) Raw Data; (d) Raw Data 


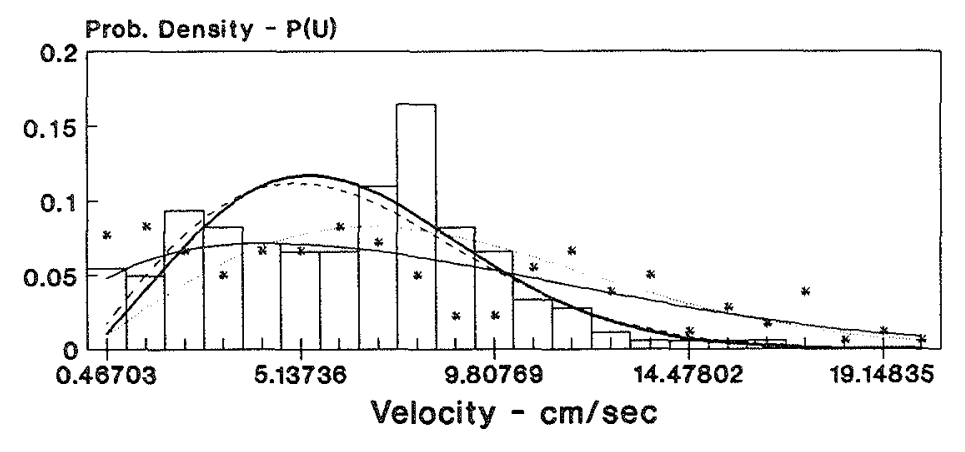

$\square$ Measured Dist. (-) … Rayleigh Dist. $\quad$ - Beta-Rayleigh

(a)

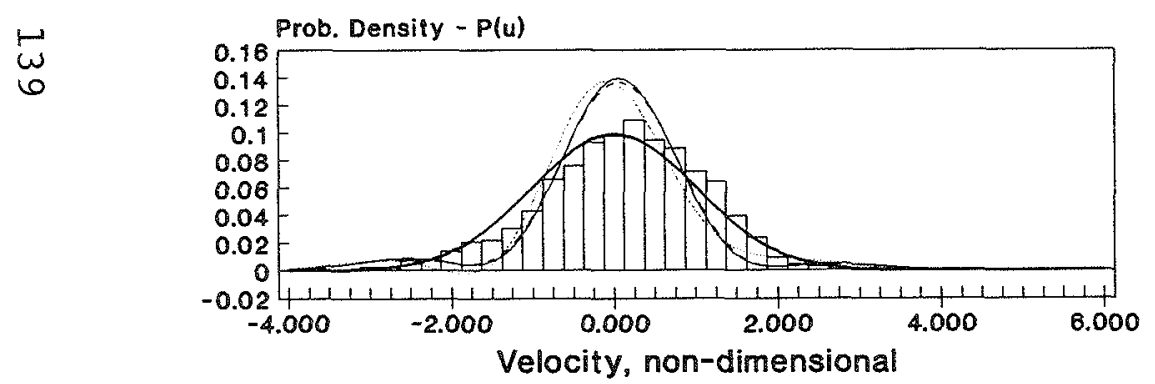

\section{Legend}

$\square$ Measured Dist. - Linear Assumption - Nontinear (i)

-.. Nonlinear (II) . Nonlinear (III)

(c)

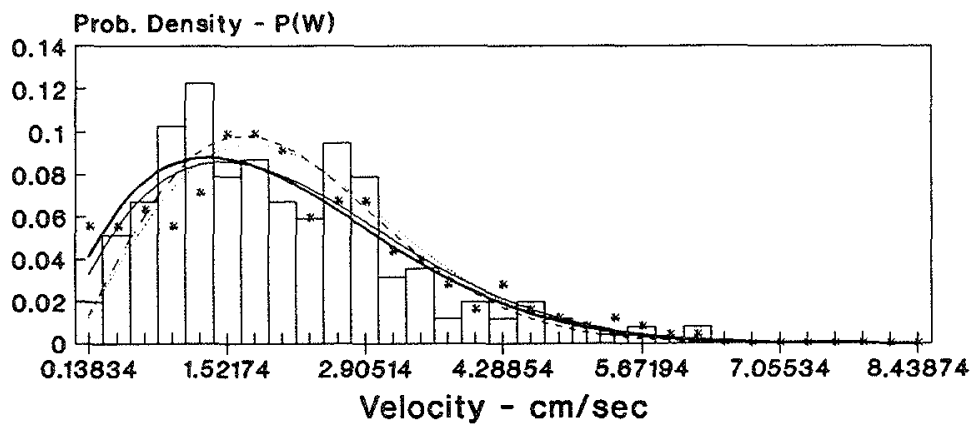

$\square$ Measured Dist. (*) -..- Rayleigh Dist. (*) - Beta-Rayleigh (*) * Measured Dist. (-) Rayleigh Dist. (-) - Beta-Rayleigh (-)

(b)

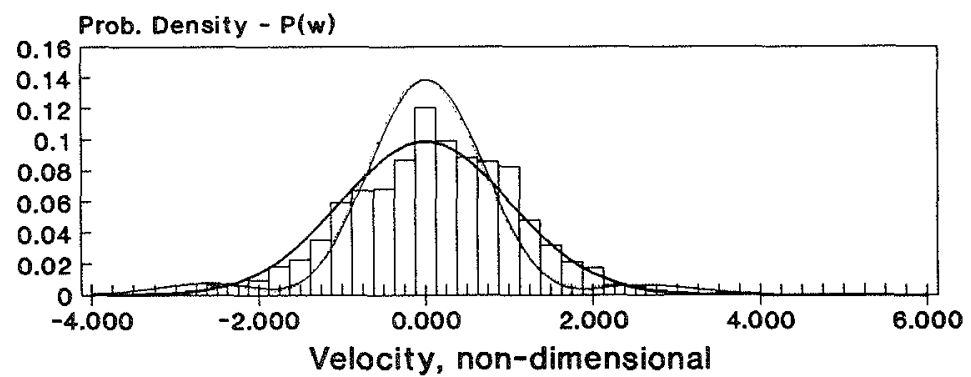

\section{Legend}

$\square$ Measured Dist. $\quad$ Linear Assumption - Nonlinear (I)

(d)

Figure 92: Time Series No. 75. (a) Filtered Data [0.20-5.00] s; (b) Filtered Data

[0.20-5.00] s; (c) Raw Data; (d) Raw Data 


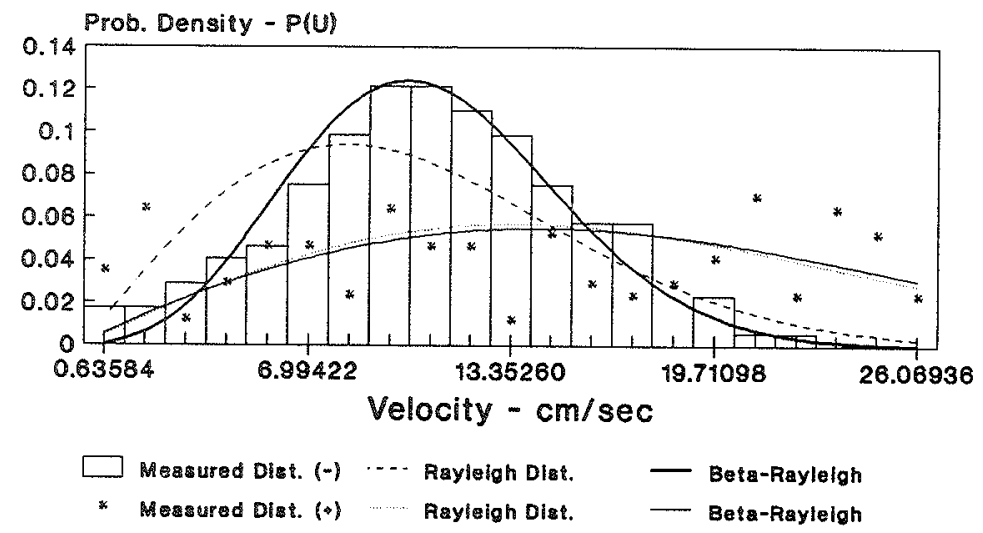

(a)
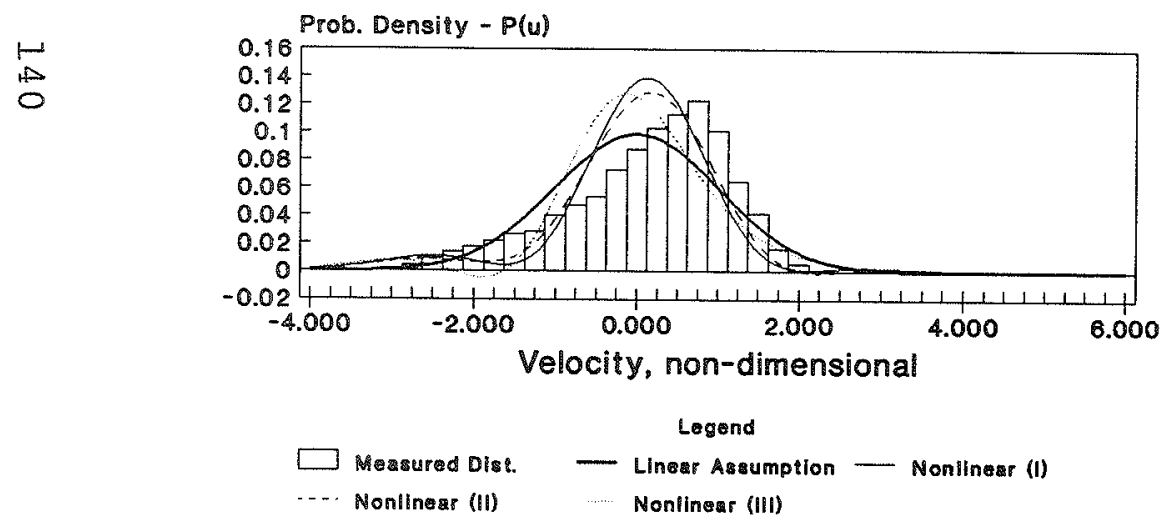

(c)

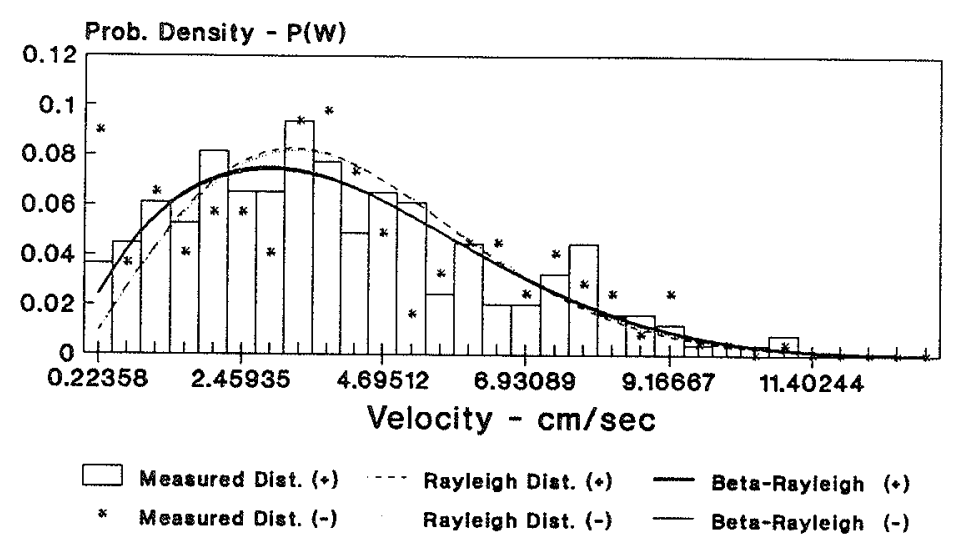

(b)
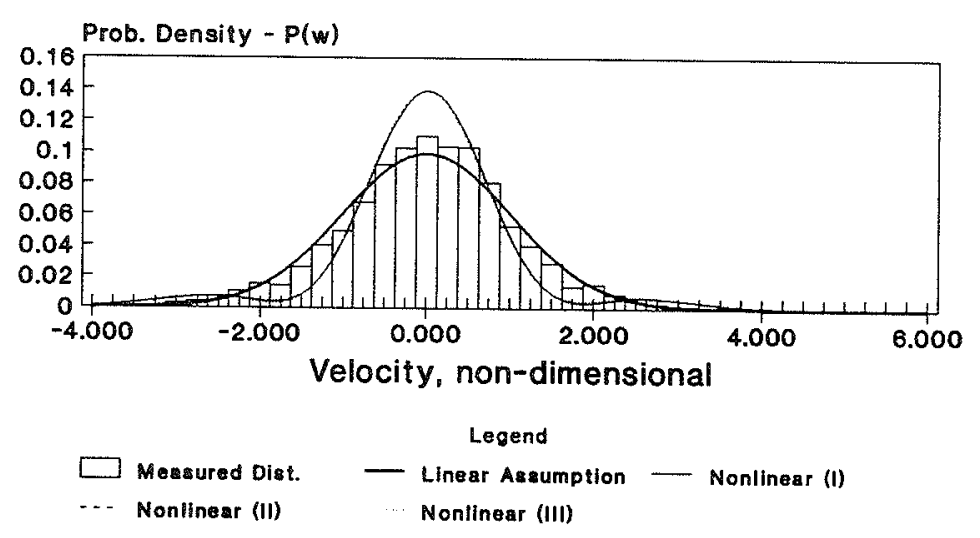

(d)

Figure 93: Time Series No. 78. (a) Filtered Data [0.20-5.00] s; (b) Filtered Data

[0.20-5.00] s; (c) Raw Data; (d) Raw Data 


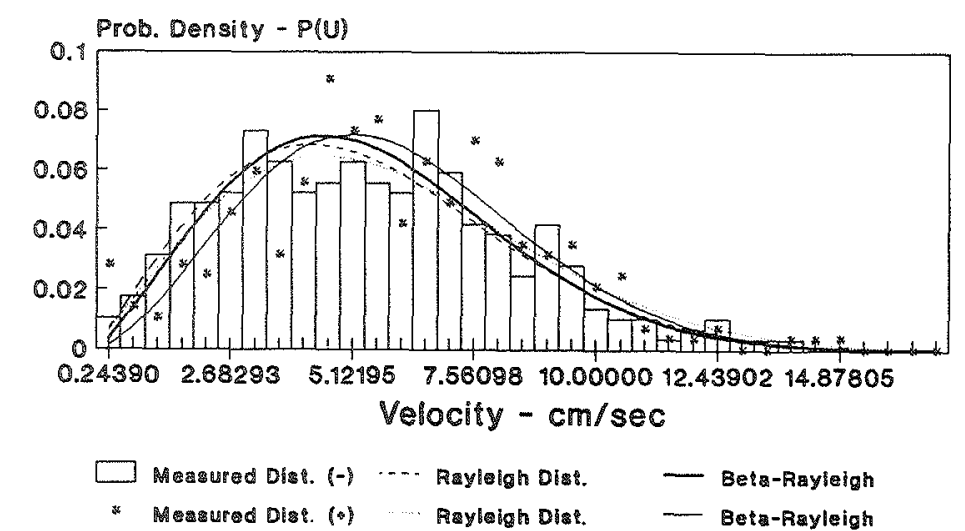

(a)

$\stackrel{\leftrightarrow}{\rightleftarrows}$

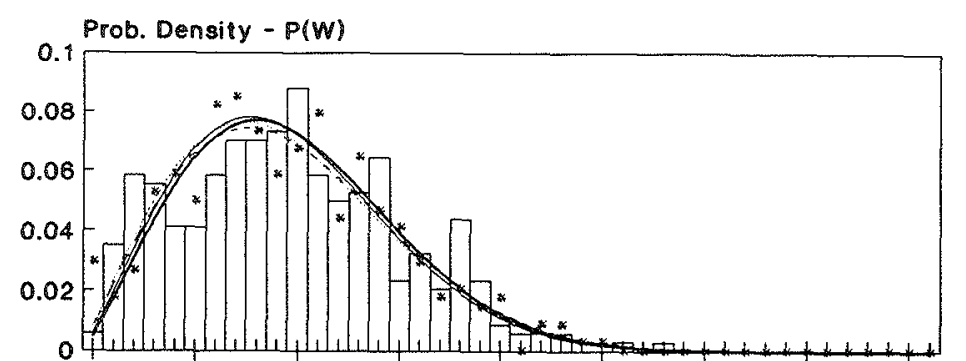

$0.058650 .645181 .23187 \quad 1.818182 .404692 .991203 .577714 .164224 .75073$ Velocity $-\mathrm{cm} / \mathrm{sec}$

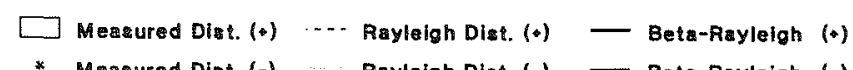

* Measured Dist. (-) … Rayleigh Dist. (-) — Beta-Aayleigh (-)

(b)

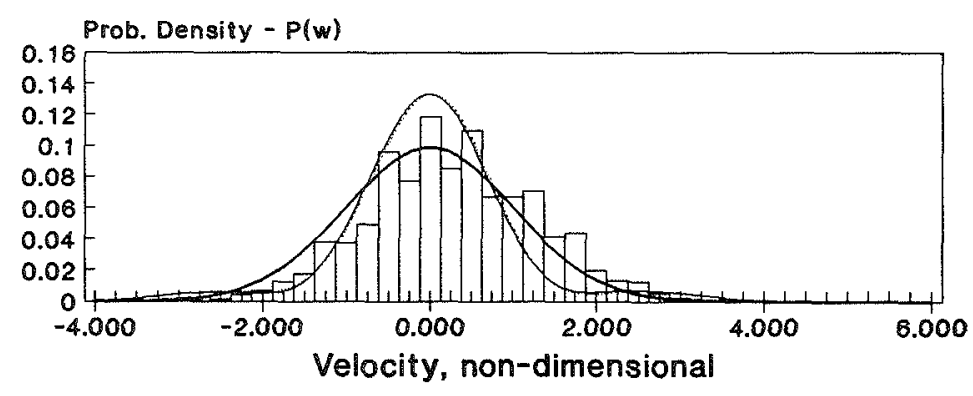

Legend

$\square$ Masured Dist. - Linear Assumption - Noninear (1) Nonlinear (II) Nonlinear (III)

(d)

Figure 94: Time Series No. 79. (a) Filtered Data [0.20-5.00] s; (b) Filtered Data

[0.20-5.00] s; (c) Raw Data; (d) Raw Data 

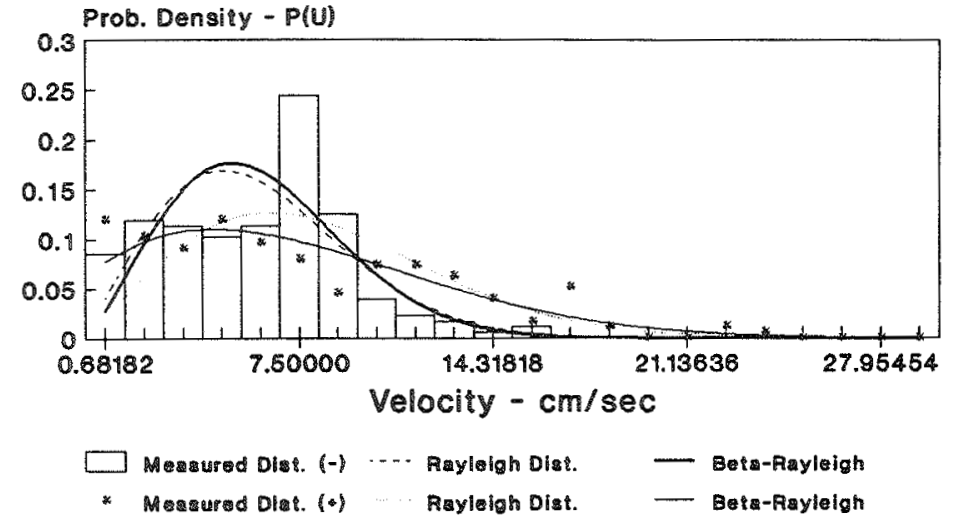

(a)
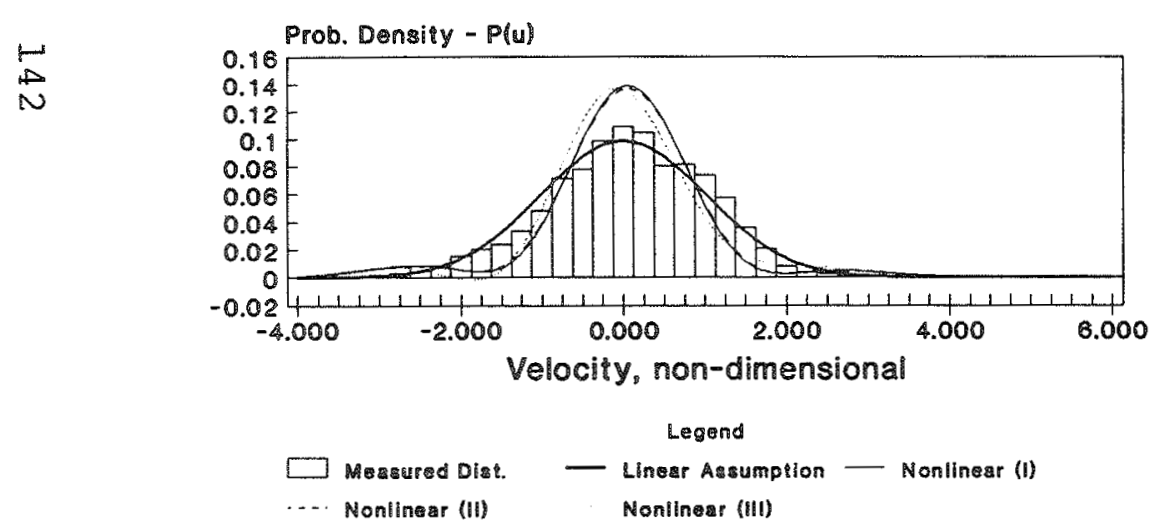

(d)

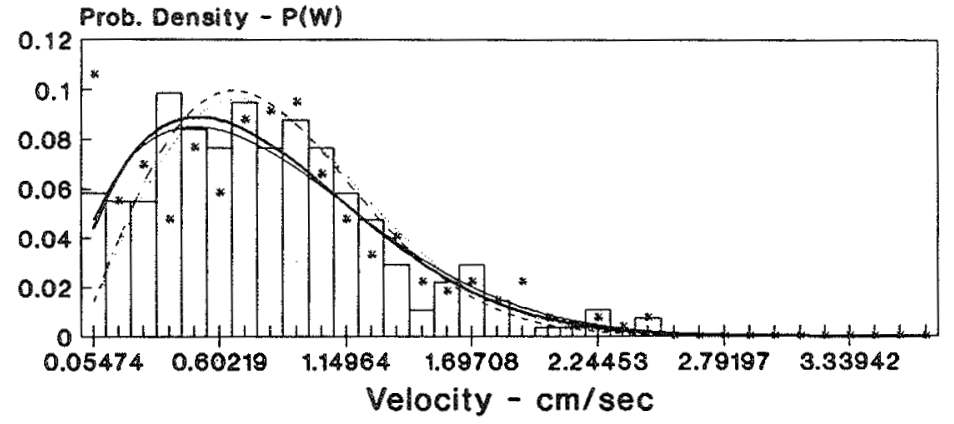

$\square$ Messured Dist. (*) -.- Rayleigh Dist. (*) — Bets-Rayleigh (*) - Musured Olst. (-) … Rayleigh Dist. (-) — Beta-Rayleigh (-)

(b)
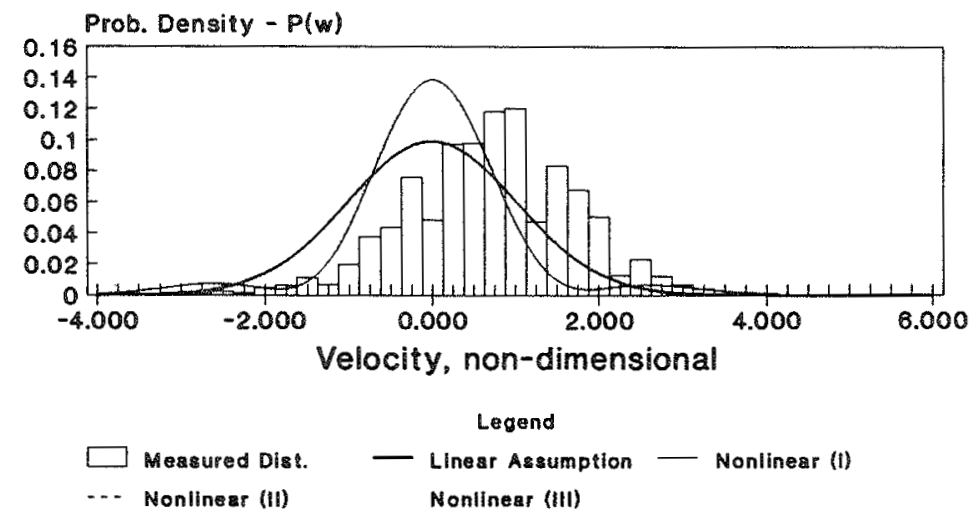

(c)

Figure 95: Time Series No. 85. (a) Filtered Data [0.20-5.00] s; (b) Filtered Data [0.20-5.00] s; (c) Raw Data; (d) Raw Data 

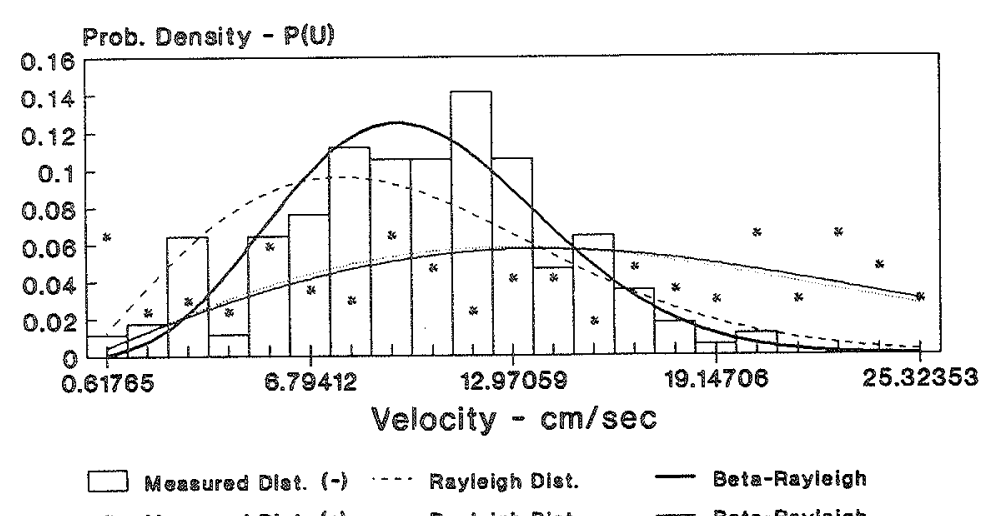

* Massured Dist. (0) …. Raylegh Dlast. - Beta-Rayieigh

(a)

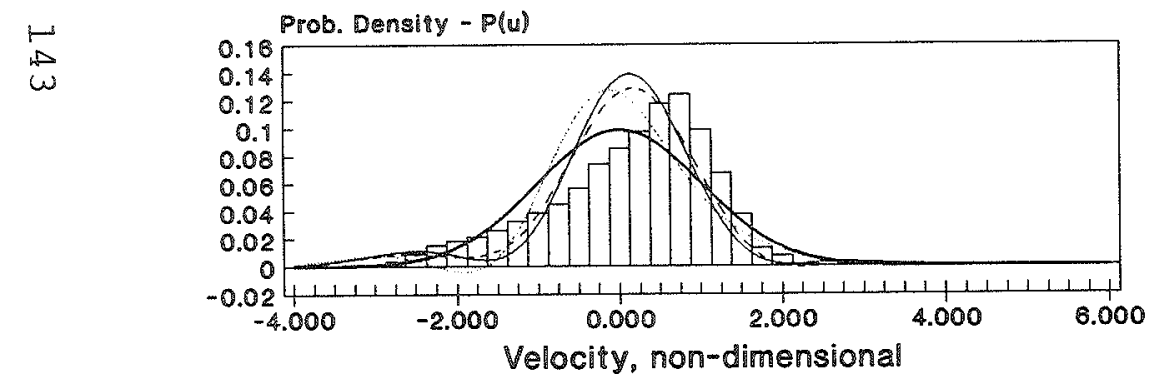

\section{Legend}

$\square$ A

(c)

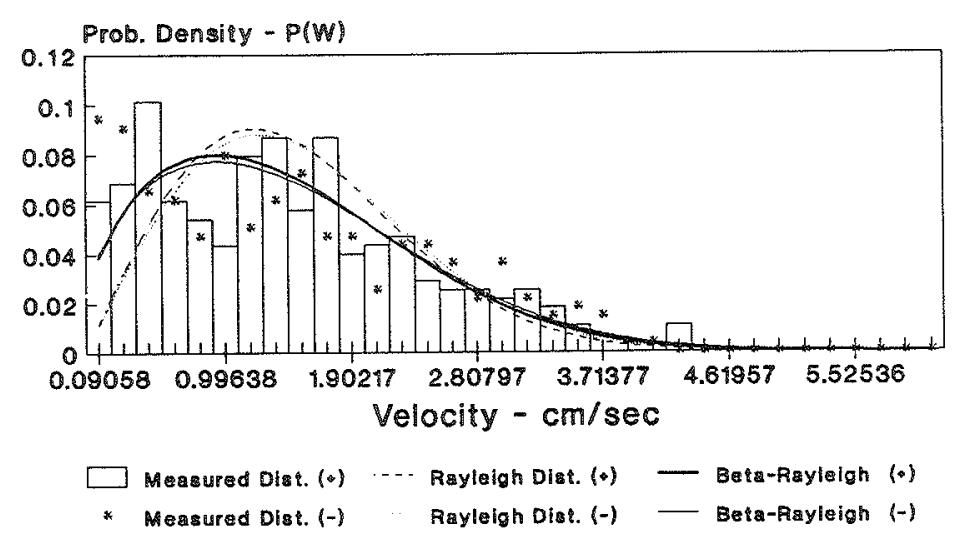

(b)

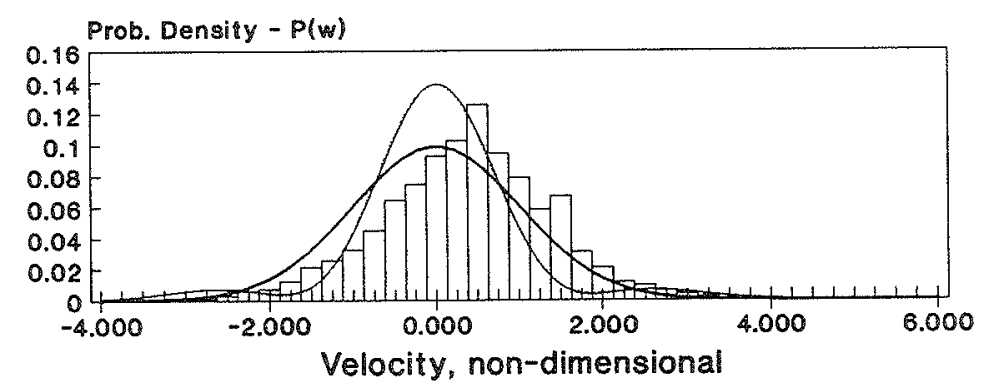

$$
\text { Legend }
$$

$\square$ measured Dist. $\quad$ Linear Assumption - Nonlinear (1) -.. Nonlinear (II) Nonlinear (III)

(d)

Figure 96: Time Series No. 88. (a) Filtered Data [0.20-5.00] s; (b) Filtered Data

[0.20-5.00] s; (c) Raw Data; (d) Raw Data 


\section{APPENDIX II: NOTATION}

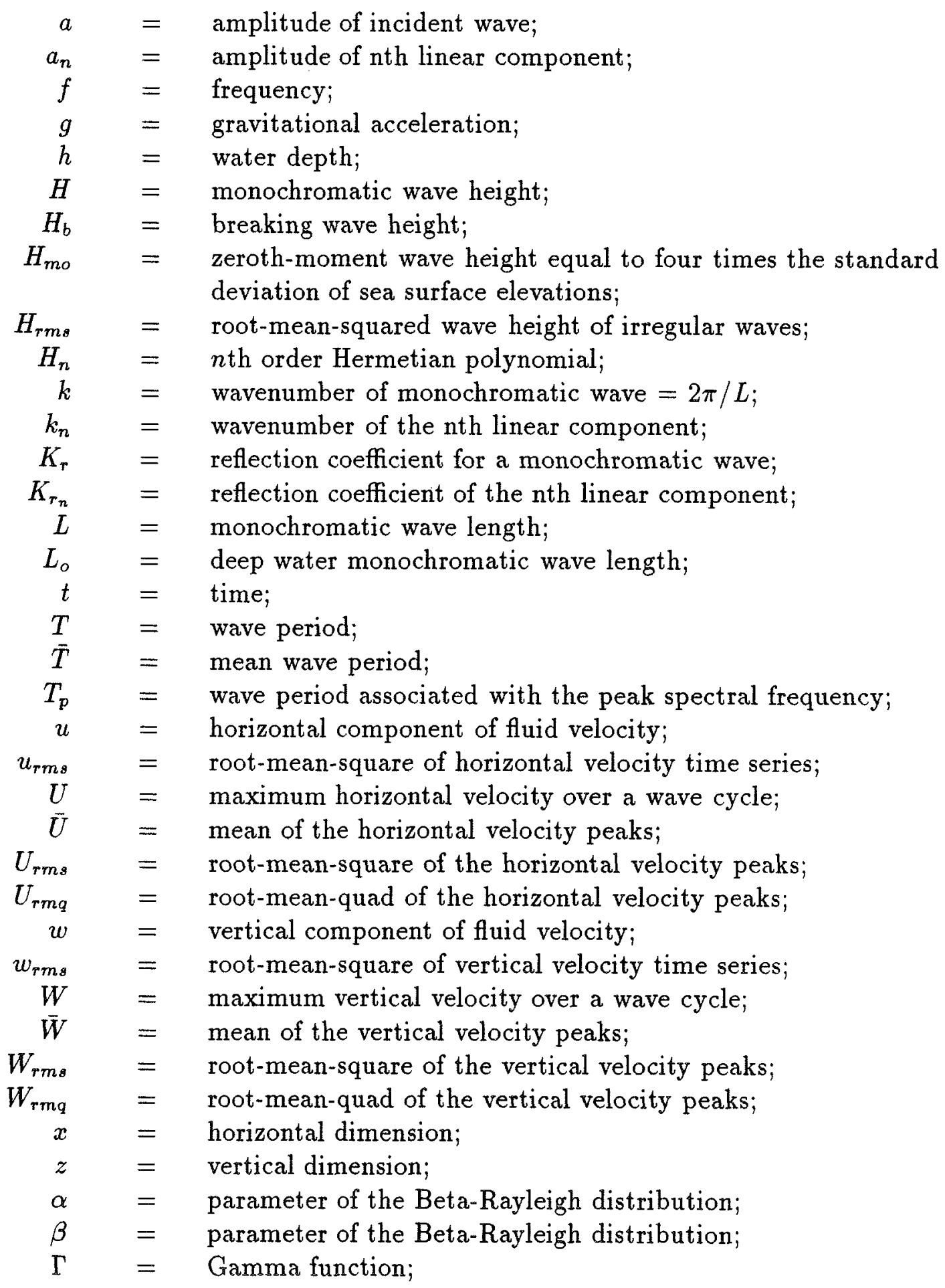




$\begin{array}{rll}\eta & = & \text { sea surface elevation; } \\ \eta_{r m s} & = & \text { root-mean-square of sea surface elevations; } \\ \theta & = & \text { phase shift due to reflection; } \\ \theta_{n} & = & \text { phase shift of the nth linear component due to reflection; } \\ \lambda_{3} & =\text { skewness; } \\ \lambda_{4} & =\text { kurtosis; } \\ \lambda_{5} & =\text { higher order moment of the nondimensional probability } \\ \pi & =\text { distribution function; } \\ \sigma & =\text { angulhematical Pi }=3.14159 \ldots ; \\ \sigma_{n} & =\text { angular wave frequency of the nth linear component. }\end{array}$

